US Army Corps

of Engineers ${ }_{\circledast}$

Engineer Research and

Development Center

\title{
Fort McCoy, Wisconsin Building 550 Maintenance Plan
}

August S. Fuelberth, Adam D. Smith, and Sunny E. Adams

November 2020

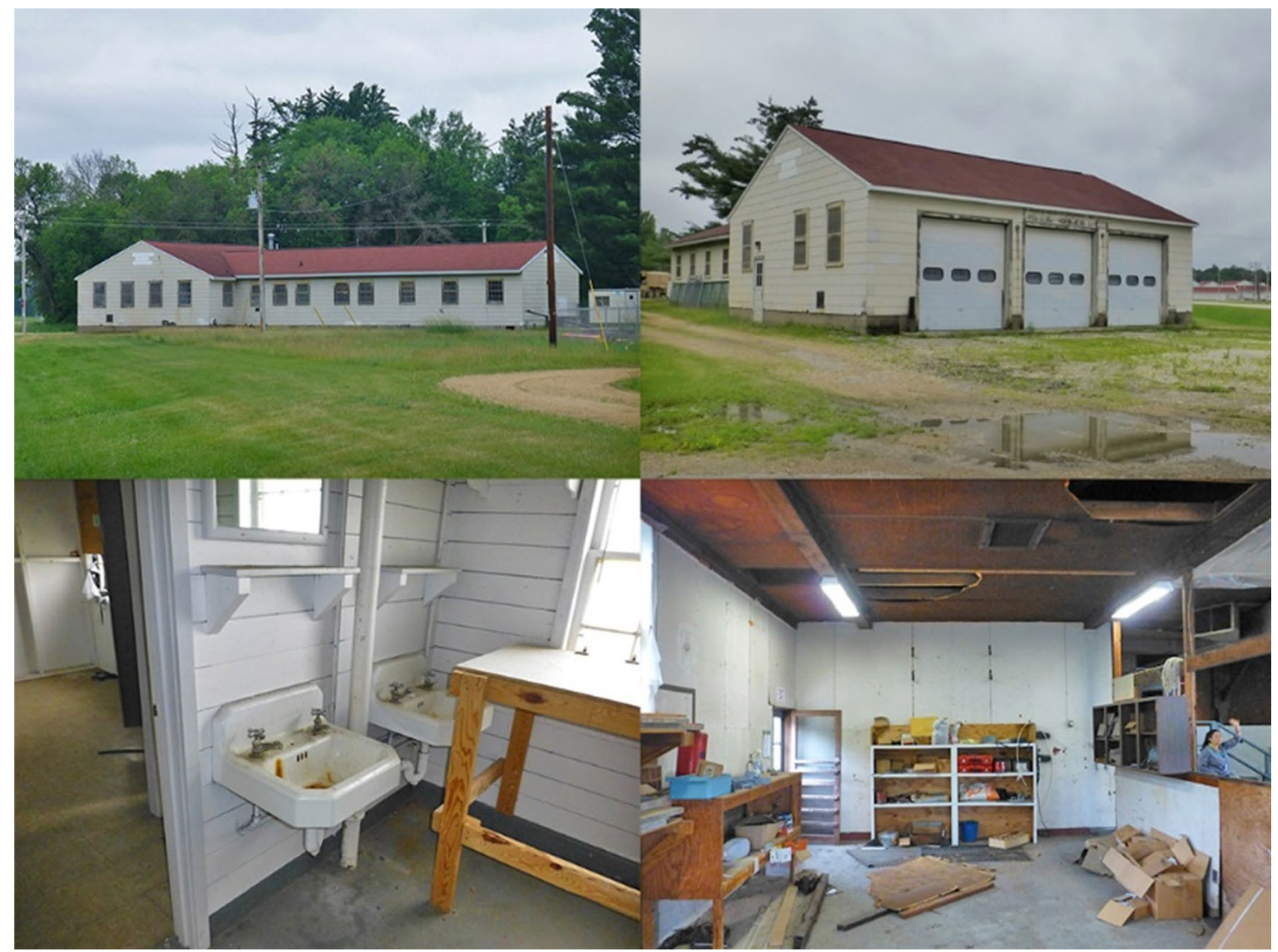


The U.S. Army Engineer Research and Development Center (ERDC) solves the nation's toughest engineering and environmental challenges. ERDC develops innovative solutions in civil and military engineering, geospatial sciences, water resources, and environmental sciences for the Army, the Department of Defense, civilian agencies, and our nation's public good. Find out more at www.erdc.usace.army.mil.

To search for other technical reports published by ERDC, visit the ERDC online library at https://erdclibrary.on.worldcat.org/discovery. 


\title{
Fort McCoy, Wisconsin Building 550 Maintenance Plan
}

\author{
August S. Fuelberth, Adam D. Smith, and Sunny E. Adams \\ U.S. Army Engineer Research and Development Center (ERDC) \\ Construction Engineering Research Laboratory (CERL) \\ 2902 Newmark Dr. \\ Champaign, IL 61824
}

Final Technical Report (TR)

Approved for public release; distribution is unlimited.

Prepared for Cultural Resource Manager

Directorate of Public Works

Fort McCoy, WI 54656

Under Project 485435, “A1010-Building 550 Maintenance Plan” 


\section{Abstract}

Building 550 (former World War II fire station) is located on Fort McCoy, Wisconsin, and was recommended eligible for the National Register of Historic Places (NRHP) in 2018 (Smith and Adams, 2018). The building is currently vacant. It is an intact example of an 800 Series World War II fire station with character-defining features of its period of significance from 1939 to 1946 on its exterior and interior. All buildings, especially historic ones, require regular planned maintenance and repair. The most notable cause of historic building element failure and/or decay is not the fact that the historic building is old, but rather it is caused by incorrect or inappropriate repair and/or basic neglect of the historic building fabric. This document is a maintenance manual compiled with as-is conditions of construction materials of Building 550. The Secretary of Interior Guidelines on rehabilitation and repair per material are discussed to provide the cultural resources manager at Fort McCoy a guide to maintain this historic building. This report satisfies Section 110 of the National Historic Preservation Act (NHPA) of 1966 as amended and will help the Fort McCoy Cultural Resources Management office to manage this historic building.

DISCLAIMER: The contents of this report are not to be used for advertising, publication, or promotional purposes. Citation of trade names does not constitute an official endorsement or approval of the use of such commercial products. All product names and trademarks cited are the property of their respective owners. The findings of this report are not to be construed as an official Department of the Army position unless so designated by other authorized documents. 


\section{Contents}

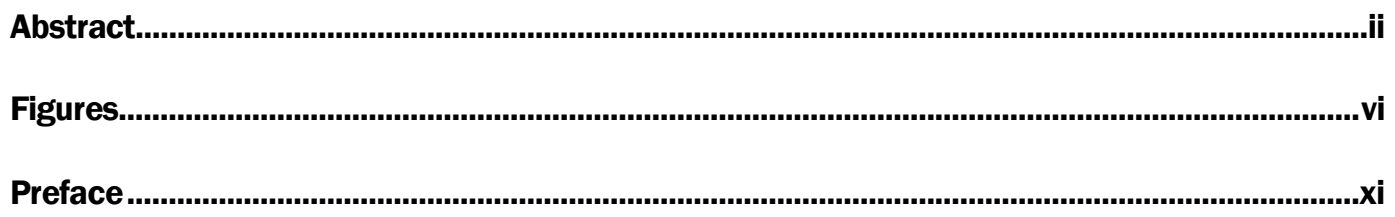

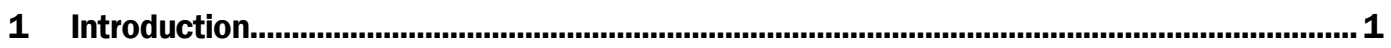

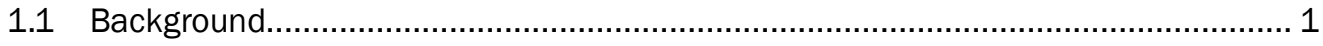

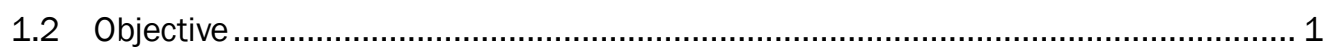

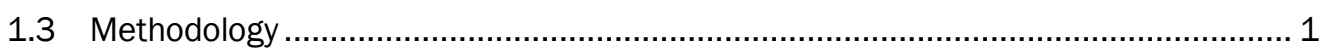

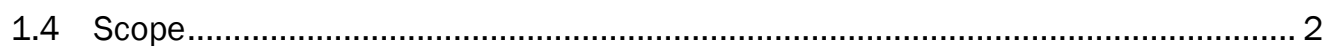

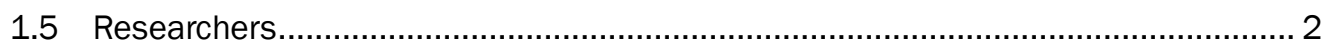

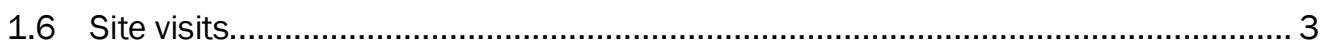

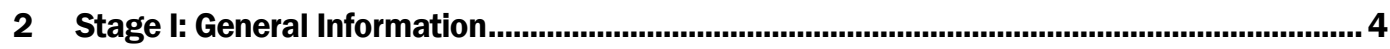

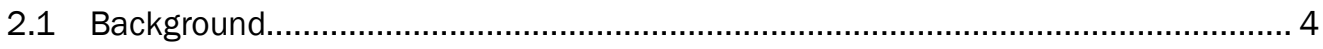

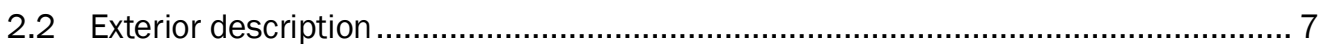

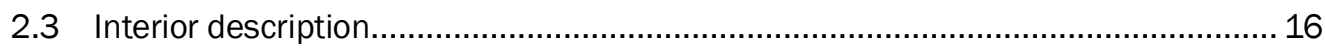

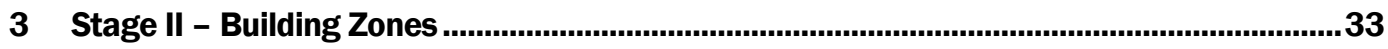

3.1 Level 2 - Preservation Zone ......................................................................... 33

3.2 Level 4 - Free Zone ............................................................................. 34

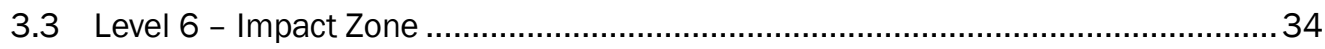

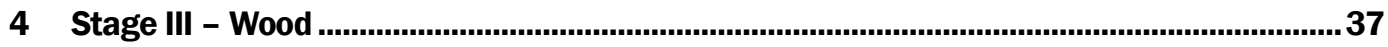

4.1 Exterior wood features ............................................................................ 37

4.2 Interior wood features................................................................................ 40

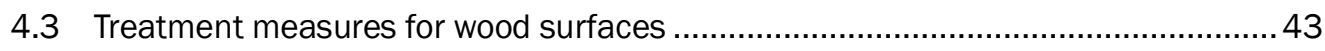

4.3.1 Preservation Brief 10: Exterior paint problem on historic woodwork, 1982

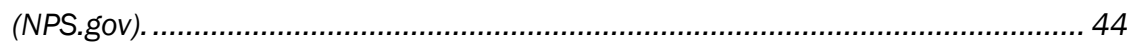

4.3.2 Replacing deteriorated woodwork, 2017 (GSA.gov)................................................ 56

4.3.3 Patching woodwork, 2017 (GSA.gov)............................................................ 59

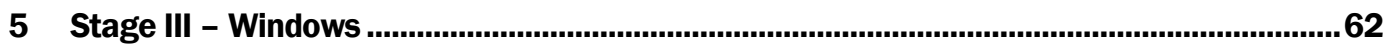

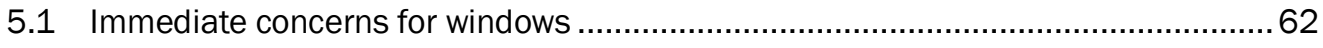

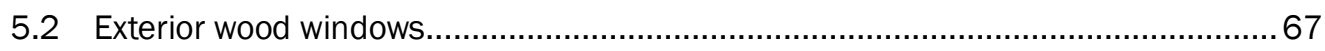

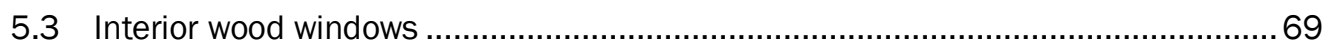

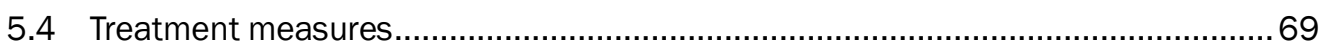

5.4.1 Preservation Brief 9: The repair of historic wooden windows, 1981 (NPS.gov)............ 70

5.4.2 Rehabilitating wood windows, 2017 (GSA.gov).................................................. 77

5.4.3 General guidelines for wood windows, 2016 (GSA.gov)........................................ 79

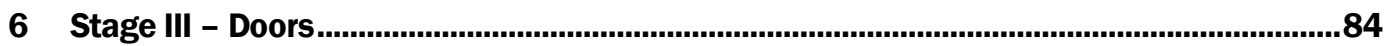

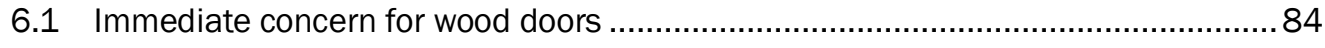

6.2 Exterior wood doors .............................................................................. 90 


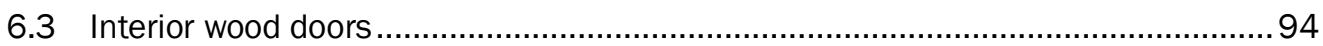

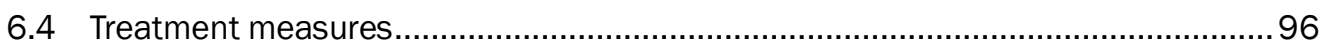

6.4.1 Stripping and refinishing stained and varnished wood doors, 2017 (GSA.gov).......... 97

6.4.2 Replacement of damaged wood doors, 2017 (GSA.gov). ....................................... 104

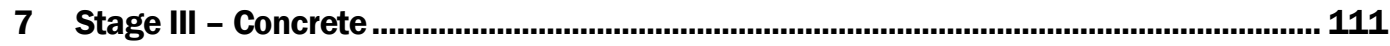

7.1 Immediate concerns for concrete .................................................................. 111

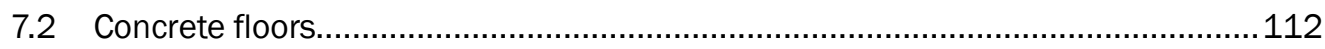

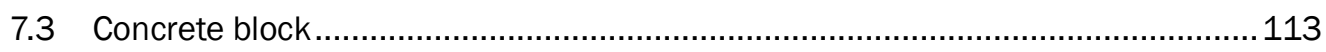

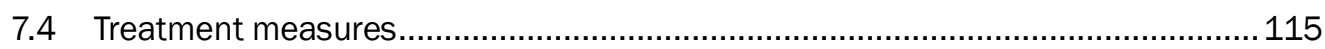

7.4.1 Types of cracks in concrete and typical causes, 2016 (GSA.gov)........................... 116

7.4.2 Removing and replacing deteriorated concrete paving, 2015 (GSA.gov).................. 119

7.4.3 Patching spalled concrete,2017 (GSA.gov)......................................................... 121

7.4.4 Treating dusting of concrete floors, 2017 (GSA.gov). .......................................... 123

7.4.5 Removing surface dirt from concrete, 2016 (GSA.gov)........................................ 127

7.4.6 Poulticing iron rust stains from concrete, 2016 (GSA.gov). ................................... 129

7.4.7 Concrete block: characteristics, uses, and problems, 2017 (GSA.gov).................... 133

7.4.8 Patching chips and cracks in ornamental concrete block, 2017 (GSA.gov)............... 135

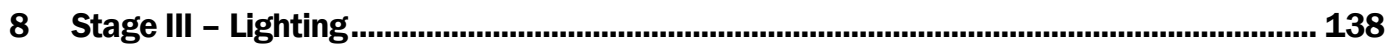

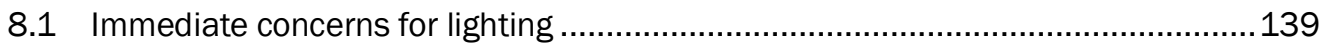

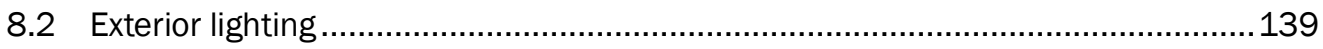

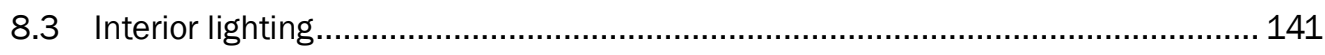

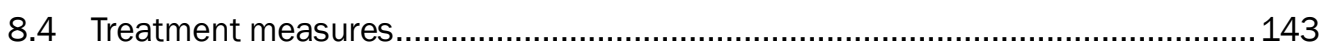

8.4.1 Technical Preservation Guidelines, 2009 (GSA.gov)........................................... 144

8.4.2 Sources of historic lighting reproductions, 2017 (GSA.gov).................................... 152

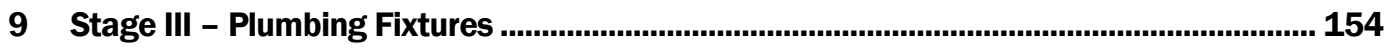

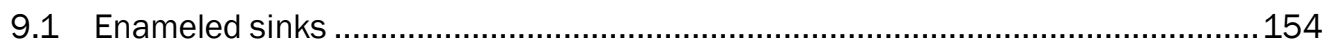

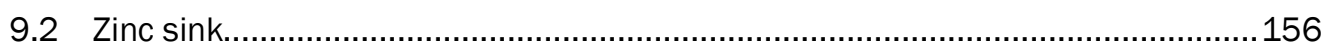

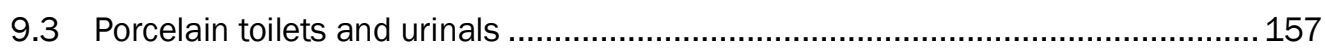

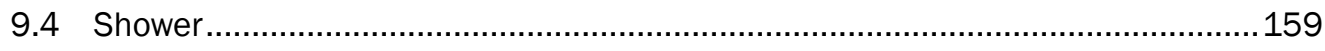

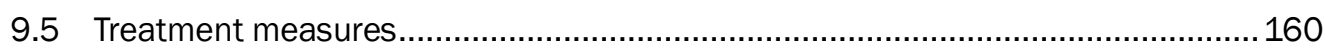

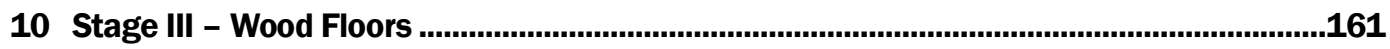

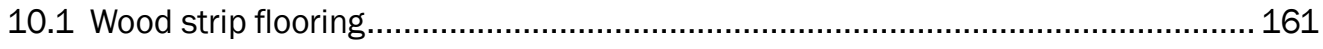

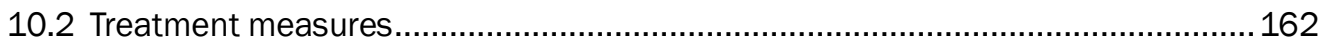

10.2.1 Stripping, staining, and polishing wood floors, GSA.gov ..................................... 163

10.2.2 Spot cleaning stains on wood floors, 2016 (GSA.gov). ....................................... 165

10.2.3 Repairing cupped floorboards, 2016 (GSA.gov) ............................................... 169

10.2.4 Replacing damaged floorboards, 2017 (GSA.gov)........................................... 172

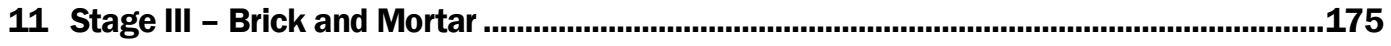

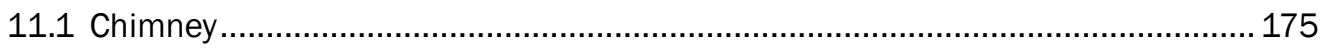

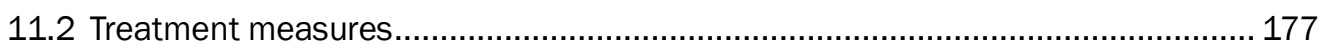

11.2.1 Preservation Brief 2 - Repointing joints in historic masonry buildings, 1998

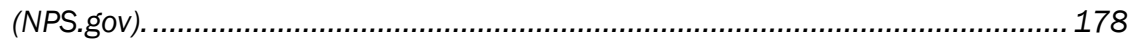


11.2.2 Guidelines for evaluating the condition of brick masonry and mortar, 2016

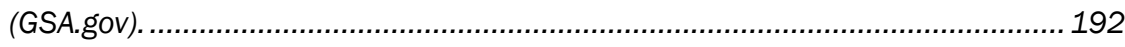

11.2.3 Removing and replacing deteriorated brick masonry, 2018 (GSA.gov). ................. 194

11.2.4 Patching cracks in brick masonry, 2018 (GSA.gov). .......................................... 198

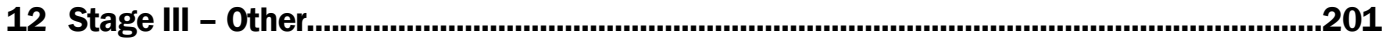

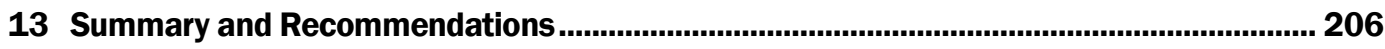

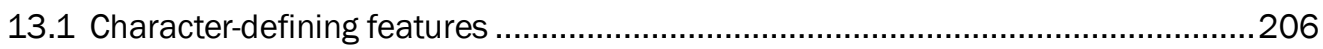

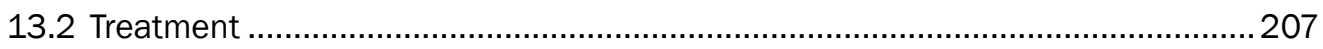

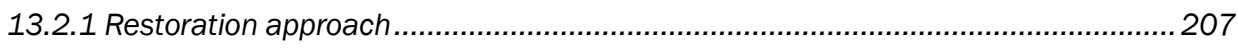

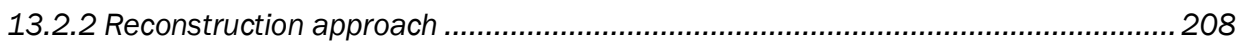

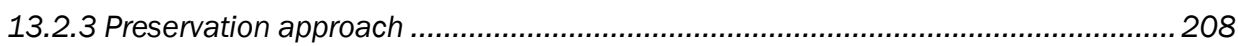

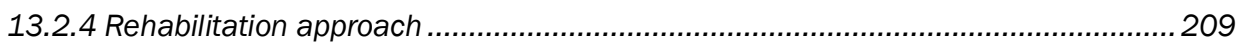

13.3 Management issues and recommendations ..................................................209

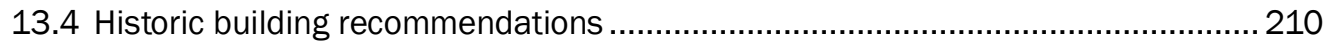

Acronyms and Abbreviations .......................................................................................... 211

Conversion Factors ............................................................................................................. 212

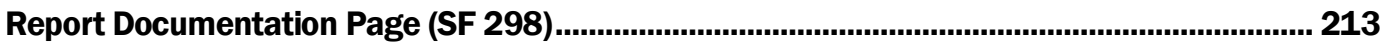




\section{Figures}

Figure

Page

1 Location map of Bldg. 550 on the northeast side of the Triad on Fort McCoy, 2014

2 Aerial map indicating the location of Bldg. 550

3 West elevation of the three-bay apparatus room of BIdg. 550, 20187

4 West elevation of the three-bay apparatus room of BIdg. 550 covered in building wrap, 2020

$5 \quad$ North elevation of the three-bay apparatus room (right side of photo) and squad room (left side of photo) of Bldg. 550, 2018

$6 \quad$ North elevation of Bldg. 550 covered in building wrap, 2020)

7 East elevation of Bldg. 550, 2018

8 East elevation of Bldg. 550 covered in building wrap, 2020

9 South elevation of the three-bay apparatus room (left side of photo) and squad room (right side of Photo) of Bldg. 550, 2018

10 South elevation of Bldg. 550 covered in building wrap, 2020

11 Northeast corner of Bldg. 550 showing the original asbestos shingle siding and the gray painted wooden foundation skirting, 2018

12 Window on the east side of the apparatus portion of Bldg. 550 showing the original eight-over-eight wood-sash double-hung window, original wooden screen and original wooden window trim, 2018

13 Close-up of the wooden trim window profile on the south side of Bldg. 550, 2018

14 Entrance door on the north side of the apparatus portion of BIdg. 550 showing the original divided-light wood panel door with original wood door trim, 2018

15 Entrance door on the east side of the squad room portion of Bldg. 550 showing the original divided-light wood panel door with original wood door trim, 2018

16 Non-original exterior light fixture above apparatus room entrance door on north side of Bldg. 550, 2018

17 Interior view of the three-bay apparatus room of Bldg. 550, looking east, 2018

18 Interior view of the three-bay apparatus room of Bldg. 550, looking south, 2018

19 Interior view of the three-bay apparatus room of Bldg. 550, looking west at two of the three replacement overhead doors, 2018 
Figure

Page

20 View of the concrete foundation wall of the apparatus room of Bldg. 550, 2018

21 Original water fountain located right outside the work room of Bldg. 550, 2018

22 Original enamel mop sink located in the work room of the three-bay apparatus room of Bldg. 550, 2018

23 Metal sink washing area located in the original alarm room of the three-bay apparatus room in Bldg. 550, 2018

24 Original cabinetry in the original alarm room of the three-bay apparatus room of Bldg. 550, 2018

25 Original enamel sink in the original alarm room of the three-bay apparatus room of Bldg. 550, 2018

26 Looking east from the three-bay apparatus room of Bldg. 550 towards the elevated living area of the building, 2018

27 Looking east down the central corridor of the living area of Bldg. 550, 2018

28 Looking west at latrine area in Bldg. 550 with original wood plank walls, exposed ceiling, concrete floor, and urinal, 2018

29 Original toilet with original wood stall walls in the latrine of Bldg. 550, 2018

30 Original toilet paper holder in Bldg. 550, 2018

31 Original sinks, wood plank walls, mirrors, and shelves in Bldg. 550, 2018

32 Original light fixture above latrine sinks in Bldg. 550, 2018

33 Original shower stall in Bldg. 550 with wood plank floor, 2018

34 Looking east at wall in small room east of latrine in Bldg. 550, 2018

35 Looking west at non-original wall dividing the squad room in half in Bldg. 550, 2018

36 Looking down at the original wood floor in the squad room in Bldg. 550, 2018

37 Looking up at the original wall board (fiber board) and batten ceiling with original ceiling vent in the squad room in Bldg. 550, 2018

38 Looking west at the original wood built-in closets in the squad room in Bldg. 550, 2018

39 Looking west at an original wood five-panel door in the latrine in Bldg. 550, 2018

40 Looking east at an original wooden screen door in Bldg. 550, 201830

41 Looking up at original duct work in the central hallway, 2018

42 Looking up at original duct work in the squad room, 2018 
Figure

43 Looking up at original ceiling vent in the squad room, 2018

44 Floor plan of Bldg. 550 and key detailing of the zones of the building (Fort McCoy DPW modified by ERDC-CERL)

45 Elevations of Bldg. 550 and key detailing of the zones of the building for Levels 2, 4, and 6

46 Looking at wooden skirting on the northeast corner of Bldg. 550, 2018

47 Detail of original plans for wooden landing, steps, and handrails on the east side of Bldg. 550, 1942

48 Looking at landing, stairs, and handrail on the east side of Bldg. 550, 2018

49 Looking at wooden window trim on the west side of Bldg. 550, 2018

50 Original painted tongue and groove walls in the toilet room in Bldg. 550, 2018

51 Wooden stair leading from the apparatus room to the squad area in Bldg. 550, 2018

52 Original wood shelves in toilet room in Bldg. 550, 2018

53 Looking up at the ceiling and wooden battens and wood trim on ceiling vent in the squad bay, 2018

54 Plan 800-151, Standard Window Details, 1942

55 Close-up detail taken from original plans for standard window Type A, 1942

56 Close-up detail taken from original plans for standard window Type B, 1942

57 Looking at a portion of "Framing Elevation C" from the original plans, 1942

58 Looking at Portion of "Framing Elevation B" from the original plans, 1942

59 Original eight-over-eight wood-sash double-hung window with wooden screen on Bldg. 550, 2018

60 Example of a "Type A" window (left) and "Type B" window (right), 2018

61 Looking at interior wood window on south side of Bldg. 550, 2018

62 Plan 800-154, Standard Door Details, 1942

63 Close-up detail of standard door “Type D," 1942

64 Close-up detail of standard door "Type C," 1942

65 Detail of wood and glass overhead doors for BIdg. 550, Plan 800801, 1942 
Figure

Page

67 Original divided-light and wood panel "Type D" door on the north elevation into the apparatus room of Bldg. 550, 2018

68 Original divided-light and wood panel "Type D" door on the east elevation leading into the squad room in Bldg. 550, 2018

69 Original set of divided-light and wood panel doors "Type C" on the north elevation into the heater room in Bldg. 550, 2018

70 Looking at original divided-light and wood door "Type D" with original interior wooden screen door on the north side of Bldg. 550, 2018

71 Replacement metal overhead doors on the west elevation of Bldg. 550, 2018

72 Looking at original wood panel interior door “Type E," 2018

73 Looking at non-original door in Bldg. 550, 2018

74 Concrete floor in the apparatus room in Bldg. 550, 2018

75 Concrete floor in the toilet room of Bldg. 550, 2018

76 Looking at poured concrete floor in poor condition, 2018

77 Concrete block is used for the heater room and the apparatus room in Bldg. 550, 2018

78 Looking at concrete block from exterior on the south side of Bldg. 550, 2018

79 Looking at concrete block from interior, 2018

80 Plan Number 800-199, Standard Electrical Details, 1942

81 Looking at an exterior original base of light fixture with original light socket, missing the bulb and shade, located on west side of Bldg. 550, 2018

82 Detail in original plans of exterior light fixtures, 1942

83 Image of replacement exterior light fixture on north side over main entrance door, 2018

84 Image of original "Type C" porcelain socket, pull-chain lights in Bldg. 550, 2018

85 Detail from original Plan Number 800-199 of light fixture, 1942

86 Looking at an original ceiling light, 2018

87 Detail from original Plan Number 800-199 of ceiling light fixture, 1942

88 Two original porcelain enameled sinks in the toilet room in Bldg. 550, 2018

89 Looking at enameled slop sink in Bldg. 550, 2018

90 Looking at enameled sink in Bldg. 550, 2018156

91 Zinc sink in the alarm room in Bldg. 550, 2018 
Figure

92 Looking at porcelain toilet in Bldg. 550 (toilet seat is not original), 2018

93 Original porcelain enameled urinal in the toilet room in Bldg. 550, 2018

94 Looking at shower stall in Bldg. 550, 2018160

95 Looking at wood flooring in Bldg. 550, 2018162

96 Looking at base of the brick chimney located on the east side of the apparatus room, 2018

97 Plan Number $800-800$ detail of original brick chimney extending out of the roof, 1942

98 Original asbestos shingle siding with a scalloped bottom edge on the northeast corner of Bldg. 550, 2018

99 Red three-tab asphalt shingles on Bldg. 550, 2018202

100 Wood plank walls and partition in the latrine in Bldg. 550, 2018203

101 The central hallway with wall board on the wall in Bldg. 550, 2018203

102 Looking up at original wall board and wood battens on the ceiling in the squad room, 2018

103 Looking up at original wall board and wood battens on the ceiling in the squad room, 2018

104 Looking up at original metal ductwork for the heating system in the squad room, 2018

105 Looking up at original metal ductwork for the heating system in the central hallway, 2018 


\section{Preface}

This study was conducted for the Cultural Resources Program, Natural Resource Branch, Environmental Division, Directorate of Public Works (DPW) at Fort McCoy, Wisconsin, under Project 485435, "A1010-Building 550 Maintenance Plan.” The technical monitors were Dr. Kira E. Kaufmann (Cultural Resources Manager, DPW) and Timothy Wilder (Natural Resources Branch Chief, DPW).

The work was performed by the Land and Heritage Conservation Branch, of the Installations Division, of the Engineer Research and Development Center, Construction Engineering Research Laboratory (ERDC-CERL). At the time of publication, Mr. Jedediah Alvey was Chief, Land and Heritage Conservation Branch; Ms. Michelle Hanson was Chief, Installations Division; and Mr. Andrew Nelson was the Technical Director for Buildings and Structures and Installation Systems. The Acting Deputy Director was Ms. Michelle Hanson and the Acting Director of ERDC-CERL was Dr. Kumar Topudurti.

COL Teresa A. Schlosser was Commander of ERDC, and Dr. David W. Pittman was the Director. 
THIS PAGE INTENTIONALLY LEFT BLANK 


\section{Introduction}

\subsection{Background}

All buildings, especially historic ones, require regular planned maintenance and repair. The most notable cause of historic building element failure and/or decay is not due to the fact that the historic building is old, but rather it is caused by an incorrect or inappropriate repair and/or basic neglect of the historic building fabric.

This report satisfies Section 110 of the National Historic Preservation Act (NHPA) of 1966 as amended and will help Fort McCoy in managing this historic building by prioritizing appropriate maintenance and repair that will help to:

- reduce the cost of maintenance in the long run;

- increase life expectancy of the building and its elements;

- use the building and its elements efficiently;

- increase safety and security; and

- ensure compliance with federal and Department of Defense historic preservation regulations.

\subsection{Objective}

The objective of this work was to gather building data through field inspections of Bldg. 550 at Fort McCoy, Wisconsin and to compile this data to help Fort McCoy manage this historic building by prioritizing appropriate maintenance and repair that will help to:

\subsection{Methodology}

The Bldg. 550 historic building maintenance and repair report is based on three successive steps-Stages I, II, and III-with each step providing a foundation for the next level. Two architectural historians gathered building data through field inspections. The researchers then compiled this data into the three stages described below.

- Stage I is the identification and documentation of the historic building and classification of the building so that it may be compared to others. This stage produces general identification information, including the background material necessary to establish a "frame of reference" for 
the building. It includes data on location, identification, size, codes, and related programs (see Chapter 2).

- Stage II allows organization of the building into one or more zones or areas of varying importance for historical and architectural reasons. Stage II contains descriptive information plus photographs and drawings to identify the areas (see Chapter 3).

- Stage III contains the identification, evaluation, and description of individual architectural features or elements within each zone established in Stage II (referred to as the Element Report). Stage III also identifies deficient elements and provides work recommendations and cost estimates to correct these deficiencies. The elements are organized into several divisions such as exterior, interior, or electrical. It is the data in Stage III which is most applicable to the maintenance, repair, and rehabilitation of the building (see Chapter 4).

\subsection{Scope}

The data collected for work related to this maintenance and repair report for Bldg. 550 is organized in two parts: graphic documentation and written information.

The graphic portion consists of photographs and floor plans of the building as it existed at the time of the inspection, plus the color-coded zone building plans that have been developed.

The written portion consists of the various elements of the building and potential repair/replacement options guided by the Secretary of the Interior Standards. ${ }^{1}$

\subsection{Researchers}

This project was conducted by the U.S. Army Corps of Engineers, Engineering Research Development Center, Construction and Engineering Research Laboratory (ERDC-CERL) in Champaign, Illinois. The research team included Adam D. Smith, Master of Architecture, as project manager, with 22 years of experience in military architectural history; Sunny E. Adams, Master of Architecture, as architectural historian, with 17 years of experience, and August S. Fuelberth as architectural intern and report compiler.

\footnotetext{
1 The 10 Standards can be found here: https://www.nps.gov/tps/standards/rehabilitation/rehab/stand.htm.
} 


\subsection{Site visits}

ERDC-CERL personnel made one trip to Fort McCoy to inventory the buildings in June 2018. During that week, team members gathered information and photographs of Bldg. 550. In 2020, Fort McCoy DPW staff assisted with the gathering of additional photographs and information. 


\section{Stage I: General Information}

Stage I is the general identification information, including the background material necessary to establish a "frame of reference" for the building. It includes data on location, identification, size, codes, and related programs.

\subsection{Background}

LOCATION: Bldg. 550 is located at the intersection of East 13th Avenue and East L Street, on Fort McCoy, Wisconsin. The area around the building is mostly devoid of buildings; the closest building is Bldg. 547 to the east. The ground surrounding is mostly flat (see Figures 1 and 2).

USGS:Alderwood Lake United States Quadrangle Universal Transverse Mercator Coordinates: Zone $15 \mathrm{~N}$

Easting 686108

Northing 4877319

PRESENT OWNER:

Department of Defense

Department of the Army

Fort McCoy, Wisconsin 54656

ORIGINAL USE: Fire Station

PRESENT USE: Vacant

DATE OF CONSTRUCTION: 1942

\section{SIGNIFICANCE:}

The 800 Series, and the 700 Series that preceded it, was a comprehensive set of drawings which could be used interchangeably in creating the various building types. War mobilization buildings are significant for their construction and technological innovation. Techniques such as the standardization of plans, prefabrication of units, and assembly-line approach to construction were largely pioneered in the construction of these mobilization structures.

The temporary wood buildings constructed with the 700 Series or 800 Series on Fort McCoy during World War II (WWII) between 1939 and 1946 were determined significant for the National Register 
of Historic Places (NRHP) in 1986 when the Programmatic Memorandum of Agreement (PMOA) was signed between the Department of Defense (DoD), the Advisory Council on Historic Preservation (ACHP), and the National Council of State Historic Preservation Officers (NCSHPO). In the early 1990s, this significance was reaffirmed by the writing of the "World War II and the U.S. Army Mobilization Program: A History of 700 and 800 Series Cantonment Construction" and the performance of the Historic American Buildings Survey (HABS) on a variety of the 700 and 800 Series planned buildings across the country. Many of the 800 Series of buildings at Fort McCoy were utilized for the HABS documentation.

Bldg. 550, a WWII-era fire station, is significant under Criterion A for WWII temporary building construction (1939-1946).

The design and construction of the WWII temporary building could not be linked to a specific architect due to its construction from standardized War Department temporary building plans. It does not possess high artistic values; however, the WWII temporary building in general was found to be significant for its design, construction and technological innovation under Criterion C. ${ }^{2}$

BUILDING NUMBER: 550

NRHP: Eligible

HABS/HAER: No HABS was done on a 3-bay WWII-era fire station. 
Figure 1. Location map of Bldg. 550 on the northeast side of the Triad on Fort McCoy, 2014.

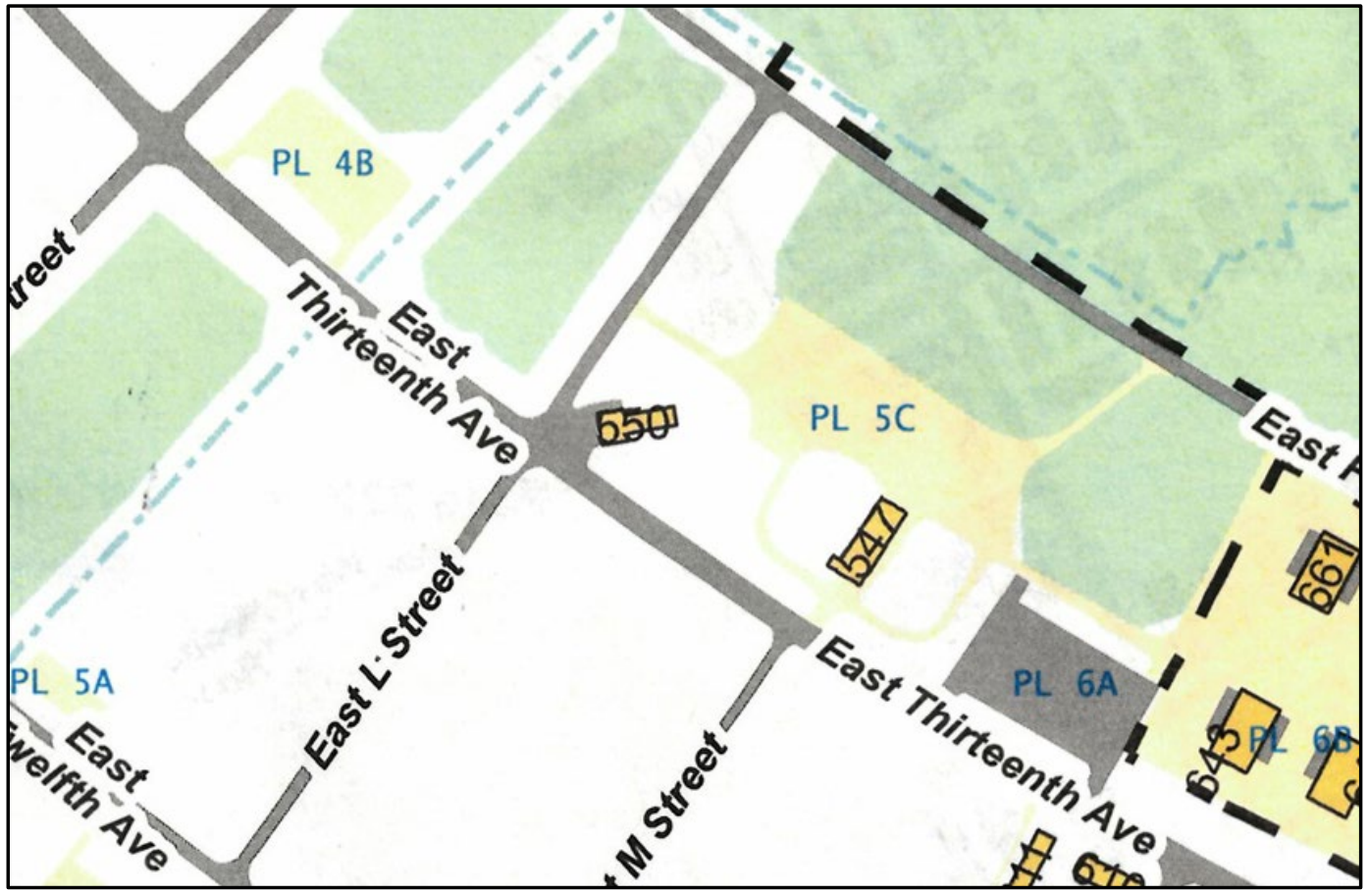

Source: Fort McCoy DPW.

Figure 2. Aerial map indicating the location of Bldg. 550.

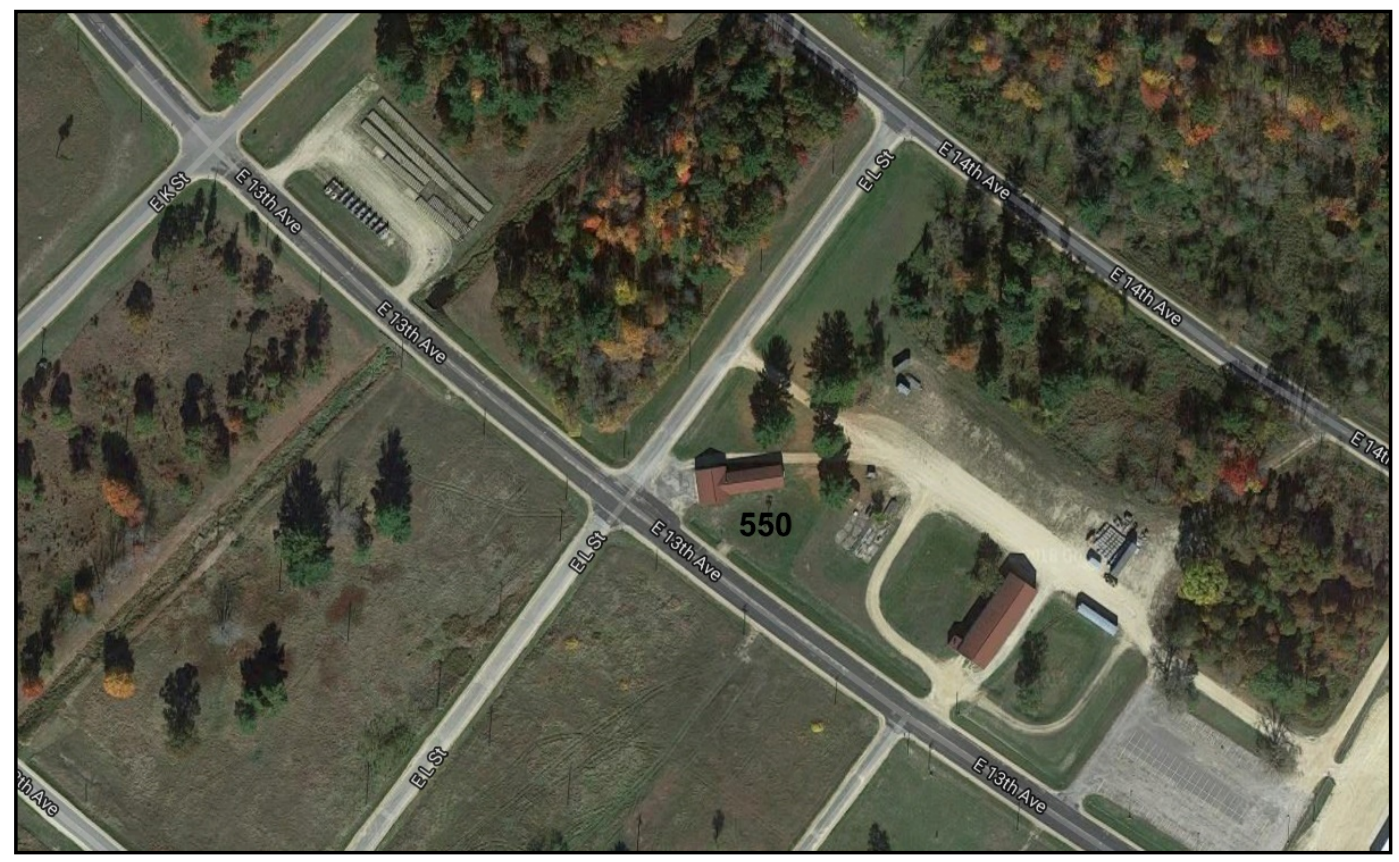

Source: google.com/maps. 


\subsection{Exterior description}

Bldg. 550 is a one-story structure with a T-shaped footprint, a cross-gable roof clad with red asphalt shingles and overhanging eaves with modified soffits, and exterior walls covered with construction building wrap added in 2019. 3 The building has an approximate square footage of 3,378 square feet.

The west elevation faces the intersection of East 13th Avenue and East L Street and consists of three replacement metal overhead doors (see Figures 3 and 4).

Figure 3. West elevation of the three-bay apparatus room of BIdg. 550, 2018.

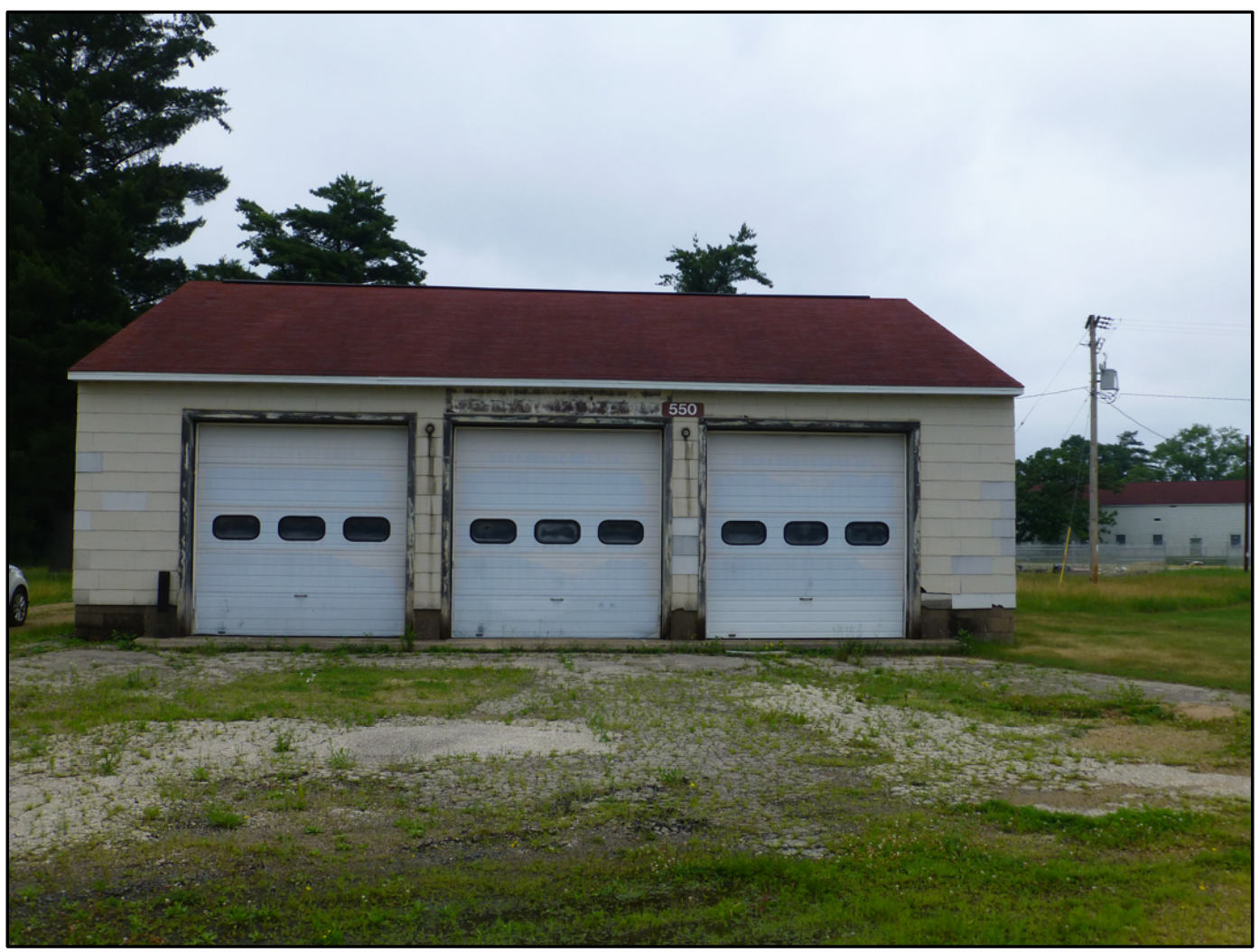

3 In 2018, when the researchers evaluated the building for the NRHP the building was clad with textured asbestos shingle siding with a scalloped bottom edge. These were removed in 2019 due to the asbestos abatement program, and the entire building was encased in building wrap except for the doors. The report includes photographs from the original visit in 2018 and ones taken in 2020 by Fort McCoy staff. 
Figure 4. West elevation of the three-bay apparatus room of Bldg. 550 covered in building wrap, 2020.

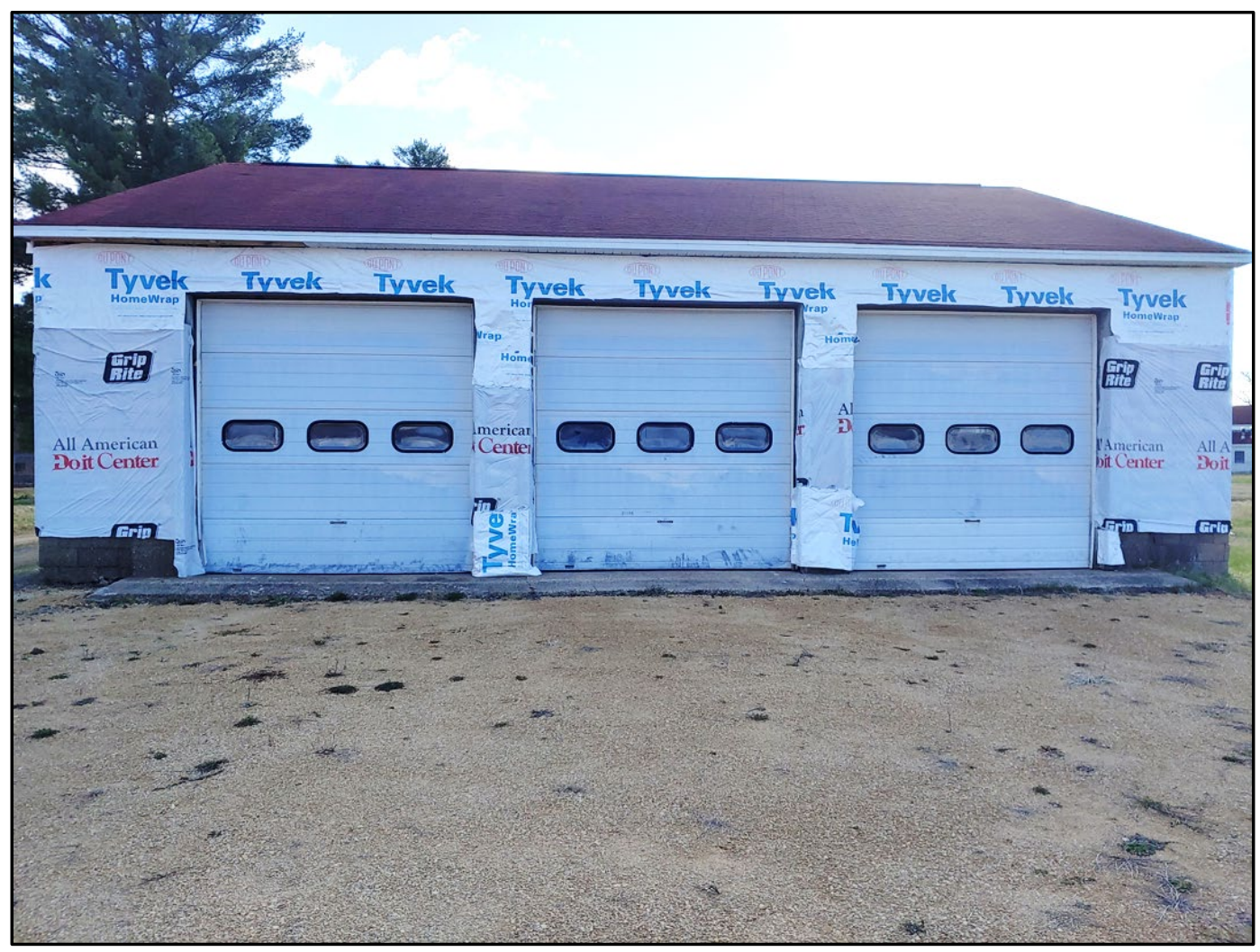

Source: Fort McCoy DPW.

The north elevation is two-part. The right side is where the three-bay apparatus room is located and projects slightly out from the left side of the elevation where the squad bay of the building is located. The right side consists of three original windows and one original entry door. The left side consists of six original windows and a set of original heater room doors (see Figures 5 and 6). 
Figure 5. North elevation of the three-bay apparatus room (right side of photo) and squad room (left side of photo) of Bldg. 550, 2018.

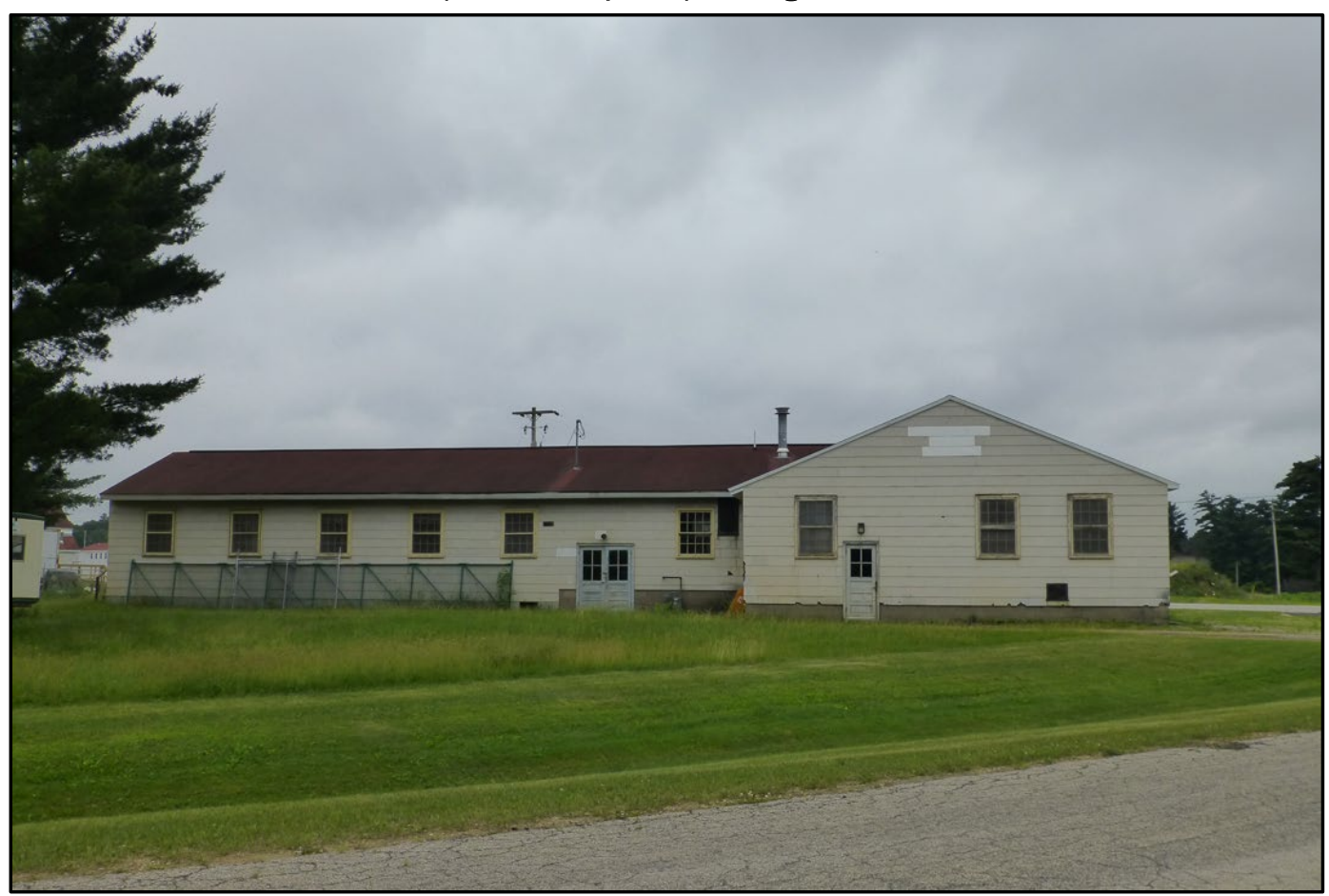

Figure 6. North elevation of Bldg. 550 covered in building wrap, 2020).

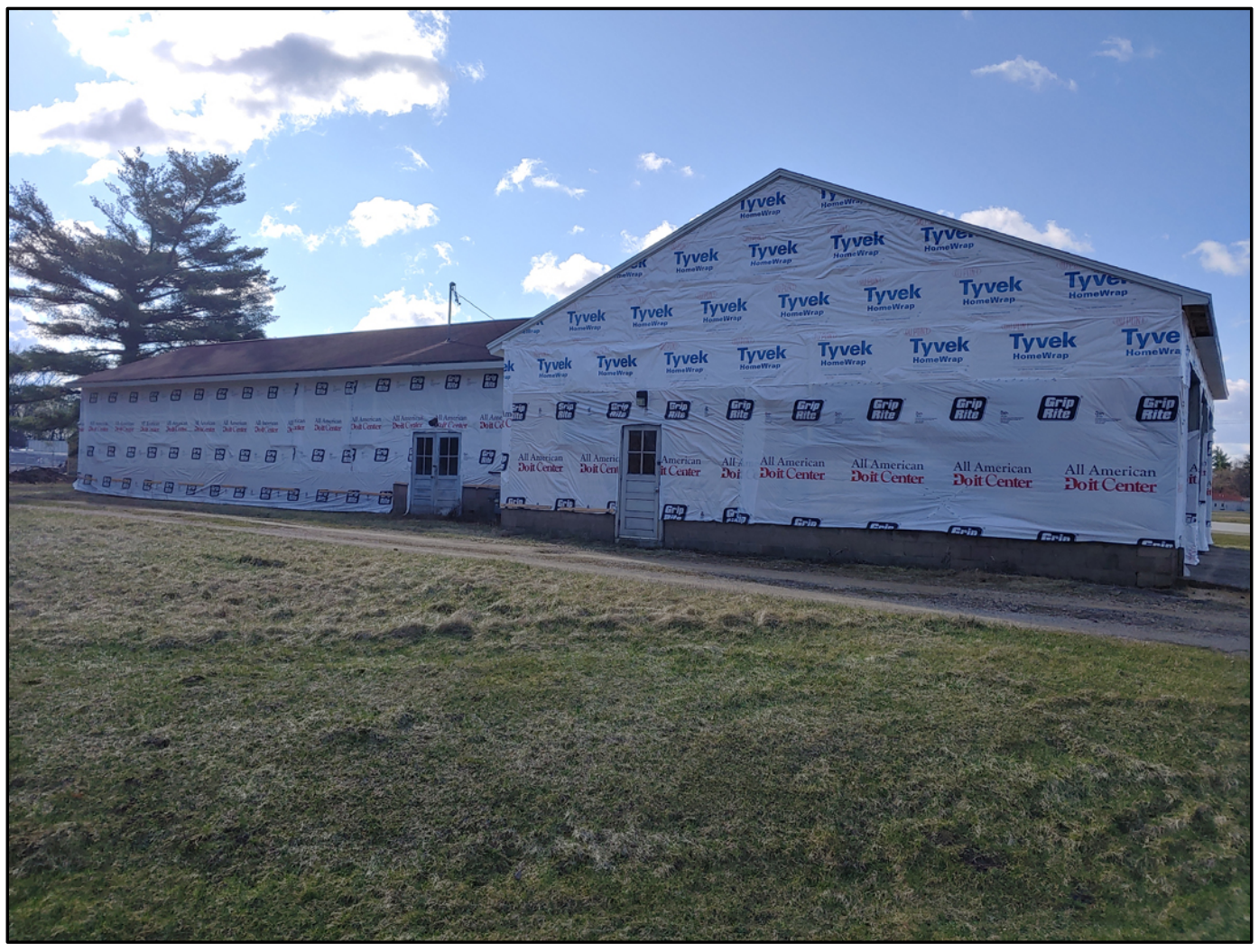

Source: Fort McCoy DPW. 
The east elevation consists of an elevated original divided-light and wood panel entry door that is accessed by a set of wood steps and wood stoop (see Figures 7 and 8). The original wood louvered vent located in the gable end has been removed.

Figure 7. East elevation of Bldg. 550, 2018.

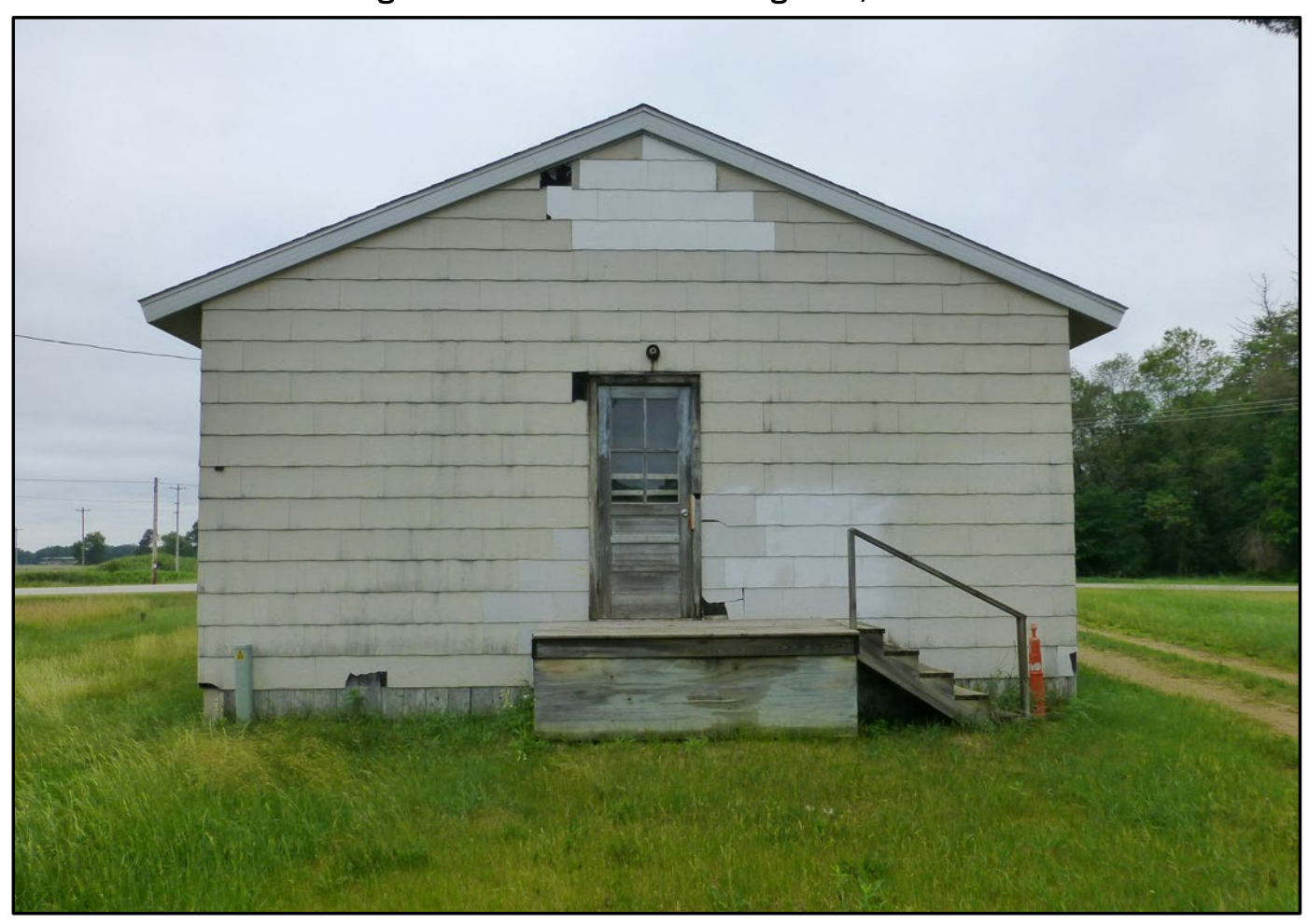

Figure 8. East elevation of Bldg. 550 covered in building wrap, 2020.

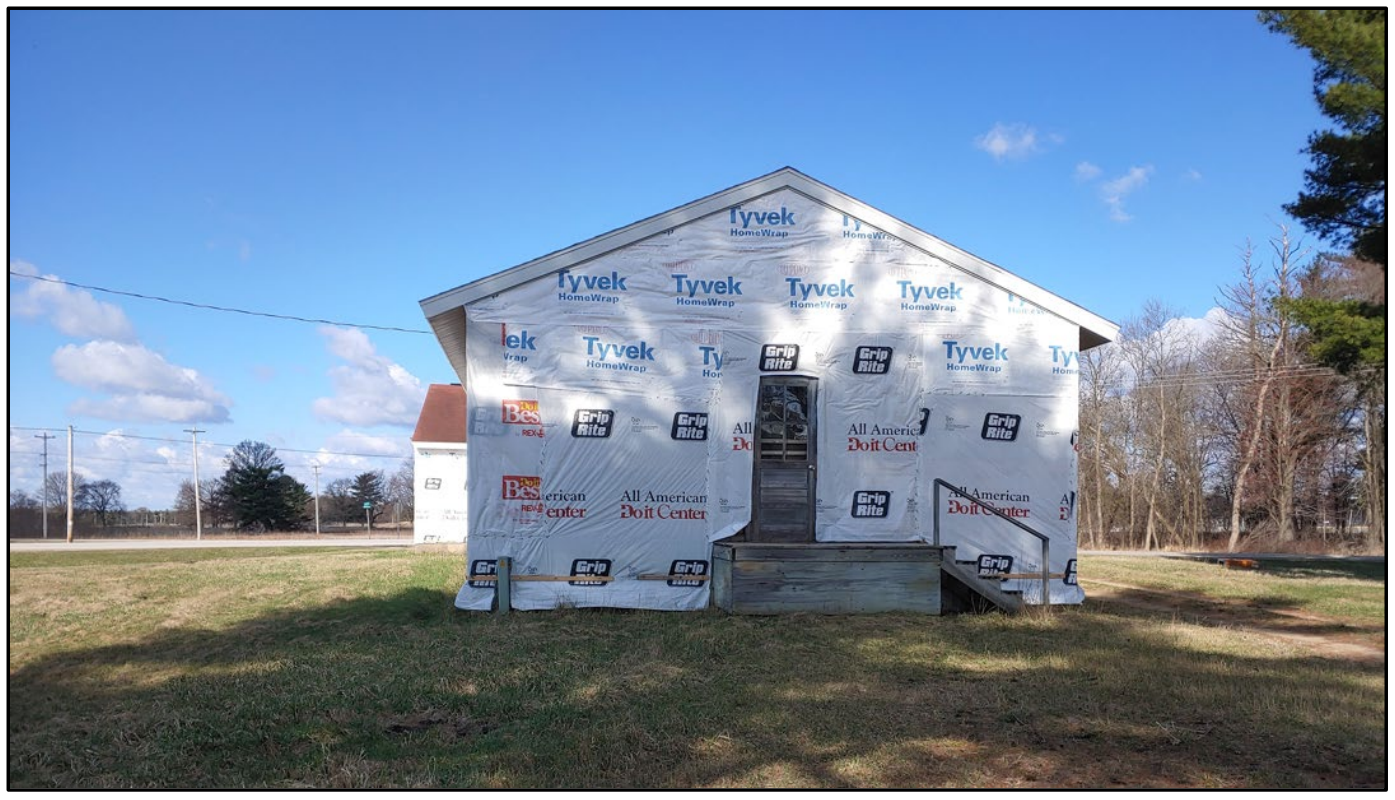

Source: Fort McCoy DPW. 
The south elevation is two-part. The left side is where the three-bay apparatus room is located and projects slightly out from the right side of the elevation where the squad room of the building is located. There are four evenly spaced original windows located on the left side of the elevation and eight original windows on the right side of the building (see Figures 9 and 10).

Figure 9. South elevation of the three-bay apparatus room (left side of photo) and squad room (right side of Photo) of Bldg. 550, 2018.

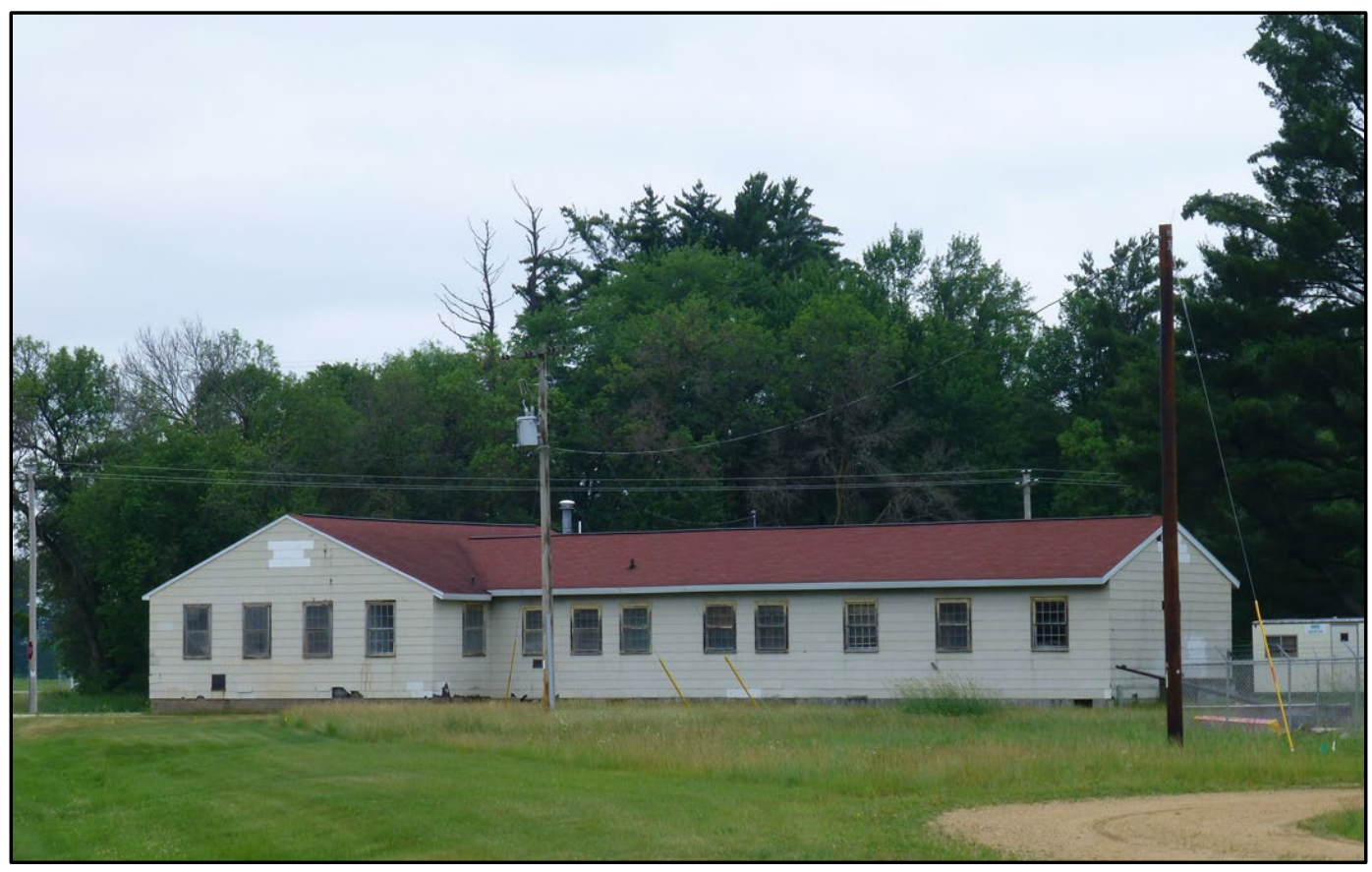

Figure 10. South elevation of Bldg. 550 covered in building wrap, 2020.

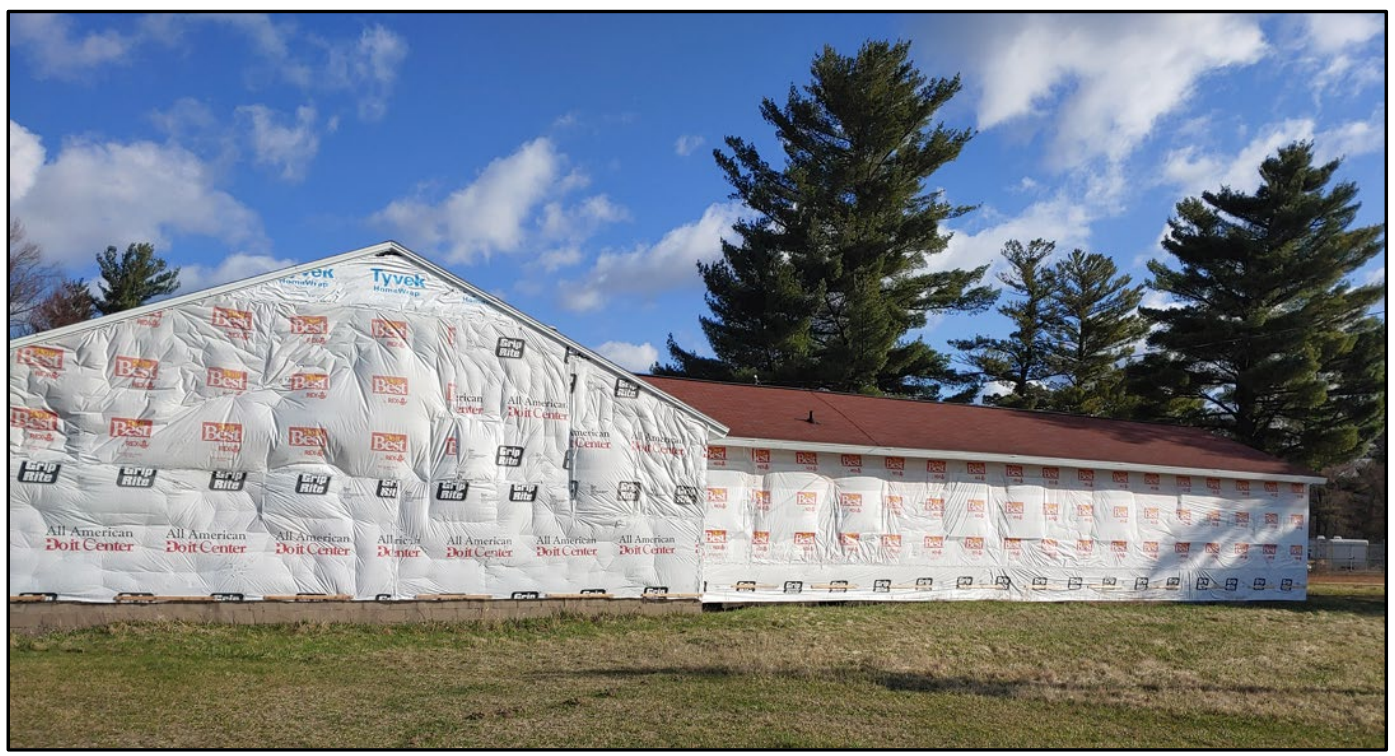

Source: Fort McCoy DPW. 
The foundation of the three-bay apparatus room and the heater room is concrete block wall, while the foundation of the living area (squad room) is a concrete pier foundation system. Original, painted gray, wood foundation "skirting" wraps around perimeter of the living area foundation (see Figure 11). The windows are original eight-over-eight wood-sash doublehung windows with wooden screens (see Figure 12). 4 The wood trim around the doors and windows are original in width and profile (see Figure 13). The entry doors into the building are divided-light and wood panel and (see Figures 14 and 15). The overhead doors are replacement metal doors (see Figure 3). All of the exterior original light fixtures have been modified; either the bulbs or shade have been removed leaving only the original base intact or the fixture has been completely replaced with newer fixtures (see Figure 16).

Figure 11. Northeast corner of Bldg. 550 showing the original asbestos shingle siding and the gray painted wooden foundation skirting, 2018.

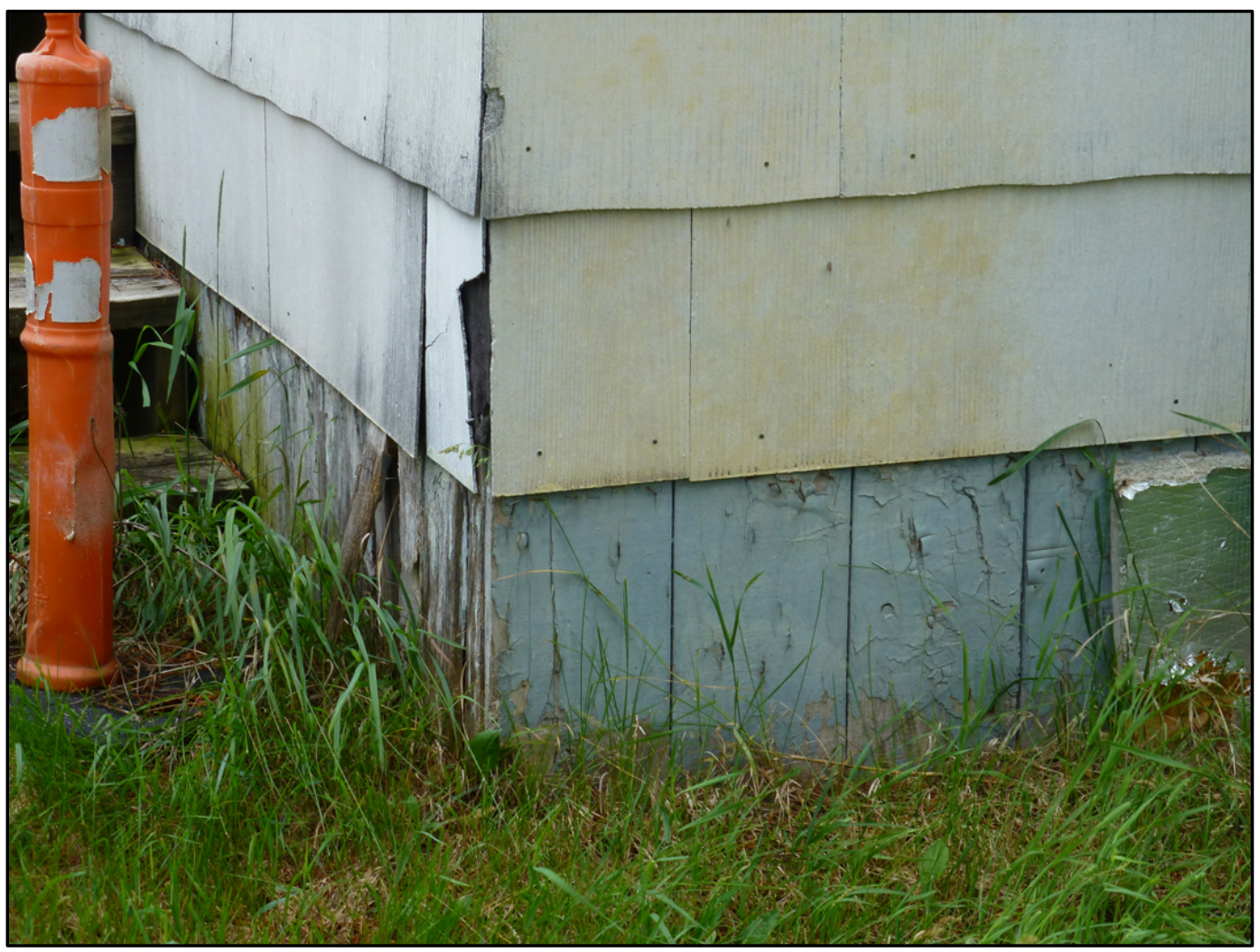

4 The windows, window screens, and window wood trim were examined and photographed in 2018. They are not viewable under the building wrap, but were untouched during the removal of the asbestos shingle siding and covering by the building wrap. 
Figure 12. Window on the east side of the apparatus portion of Bldg. 550 showing the original eight-over-eight wood-sash double-hung window, original wooden screen and original wooden window trim, 2018.

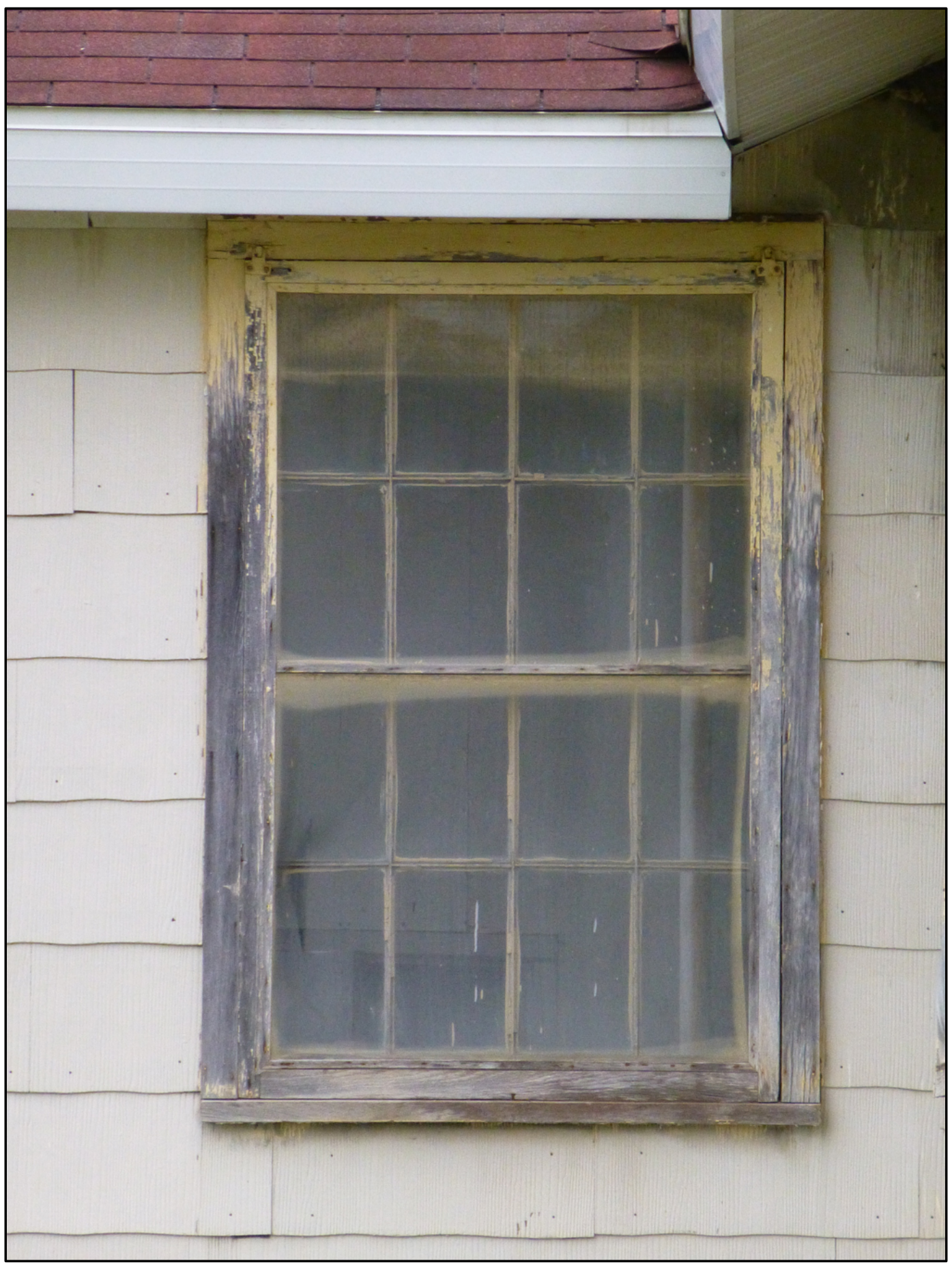


Figure 13. Close-up of the wooden trim window profile on the south side of Bldg. 550, 2018.

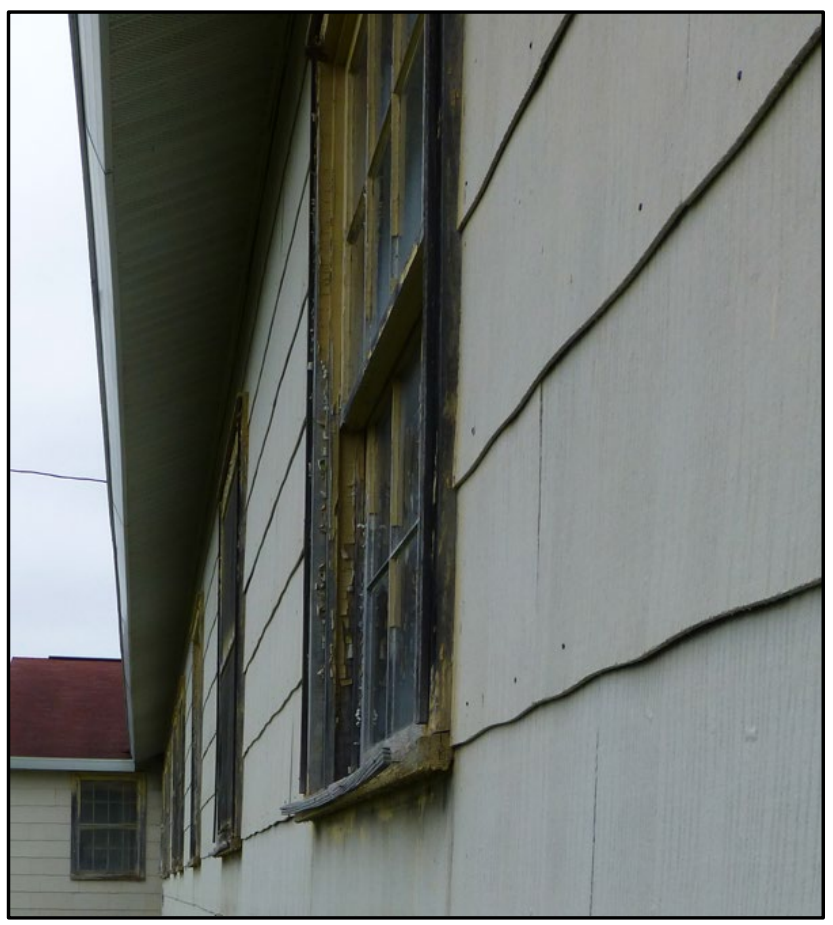

Figure 14. Entrance door on the north side of the apparatus portion of Bldg. 550 showing the original divided-light wood panel door with original wood door trim, 2018.

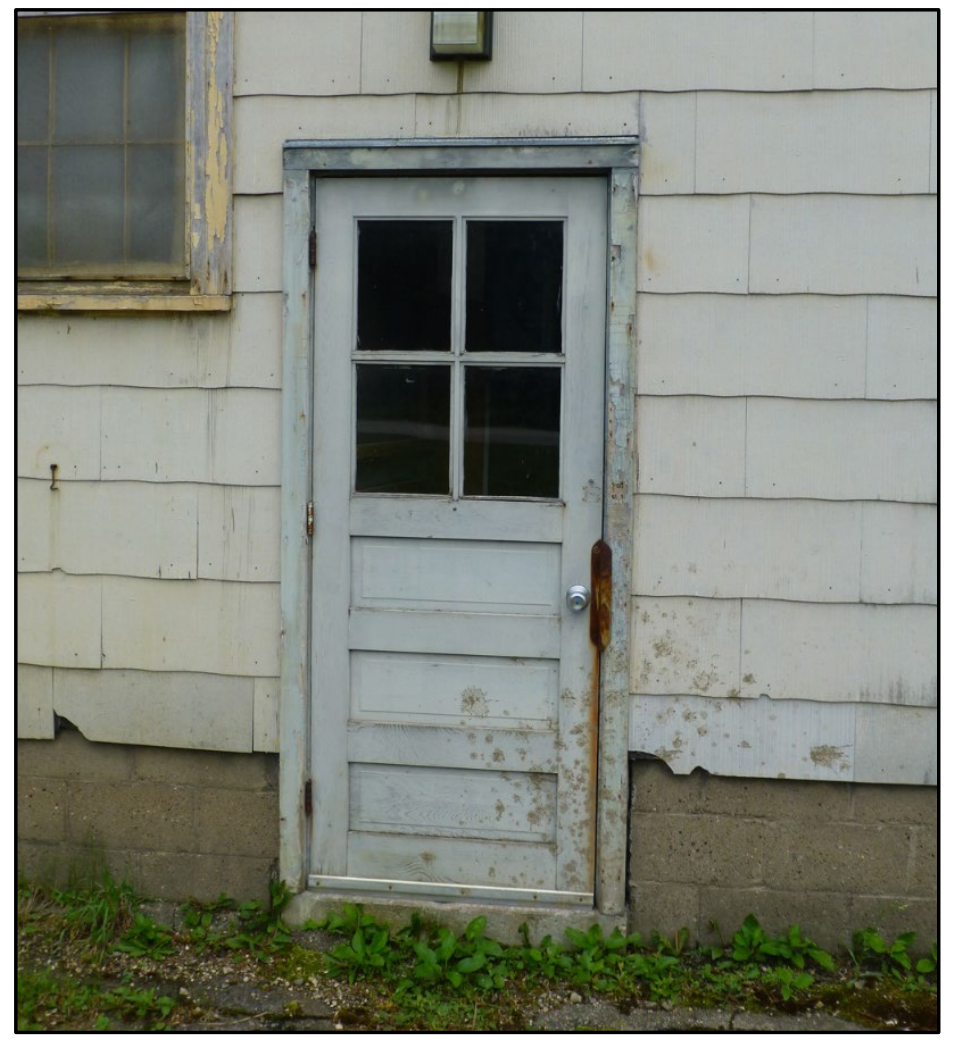


Figure 15. Entrance door on the east side of the squad room portion of Bldg. 550 showing the original divided-light wood panel door with original wood door trim, 2018.

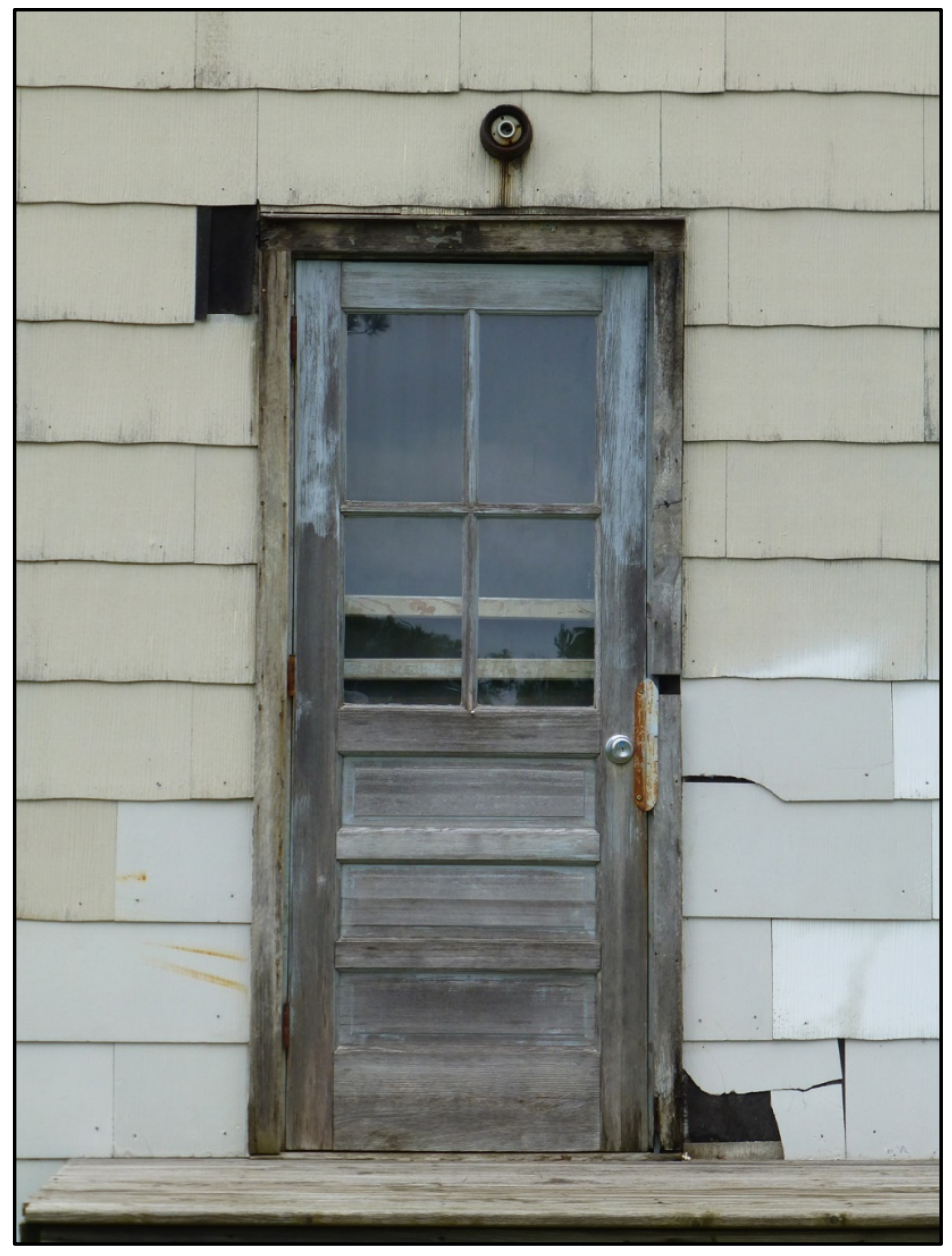

Figure 16. Non-original exterior light fixture above apparatus room entrance door on north side of Bldg. 550, 2018.

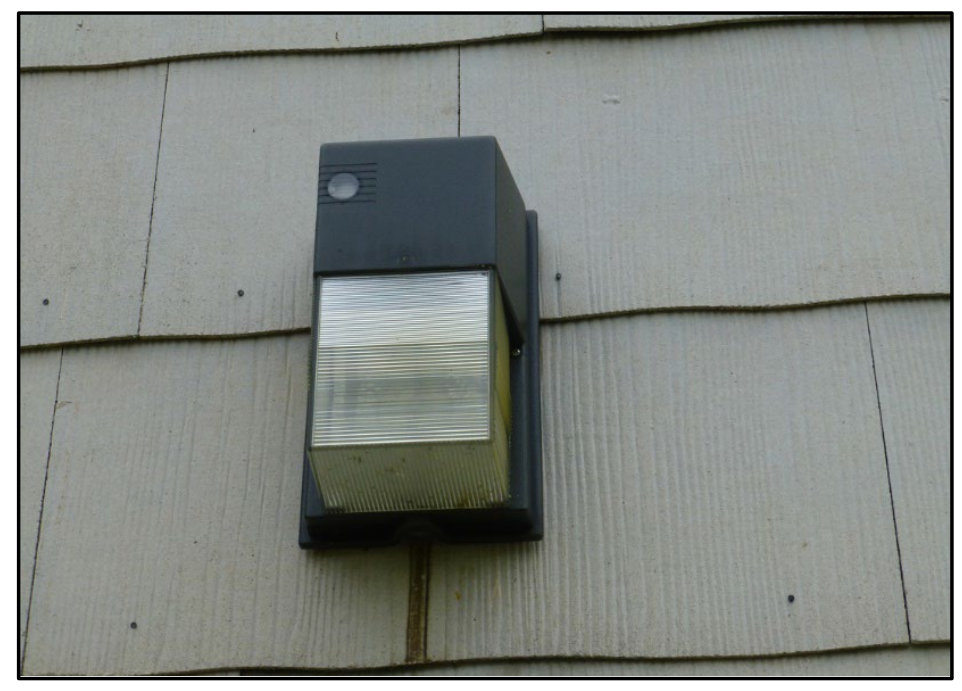




\subsection{Interior description}

The interior layout and space usage of the building has seen little alteration. The open bay area of the apparatus room with concrete floor and gypsum clad walls and ceiling are intact (see Figures 17 through 20). The two small rooms within the apparatus room; the work room with original mop sink and water fountain right outside are intact (see Figures 21 and 22) and the alarm room with original cabinetry and sinks is intact 5 (see Figures 23 through 25). The living section (squad room) of the building is elevated above the level of the apparatus room and accessed by a set of wood stairs (see Figure 26).

Figure 17. Interior view of the three-bay apparatus room of Bldg. 550, looking east, 2018.

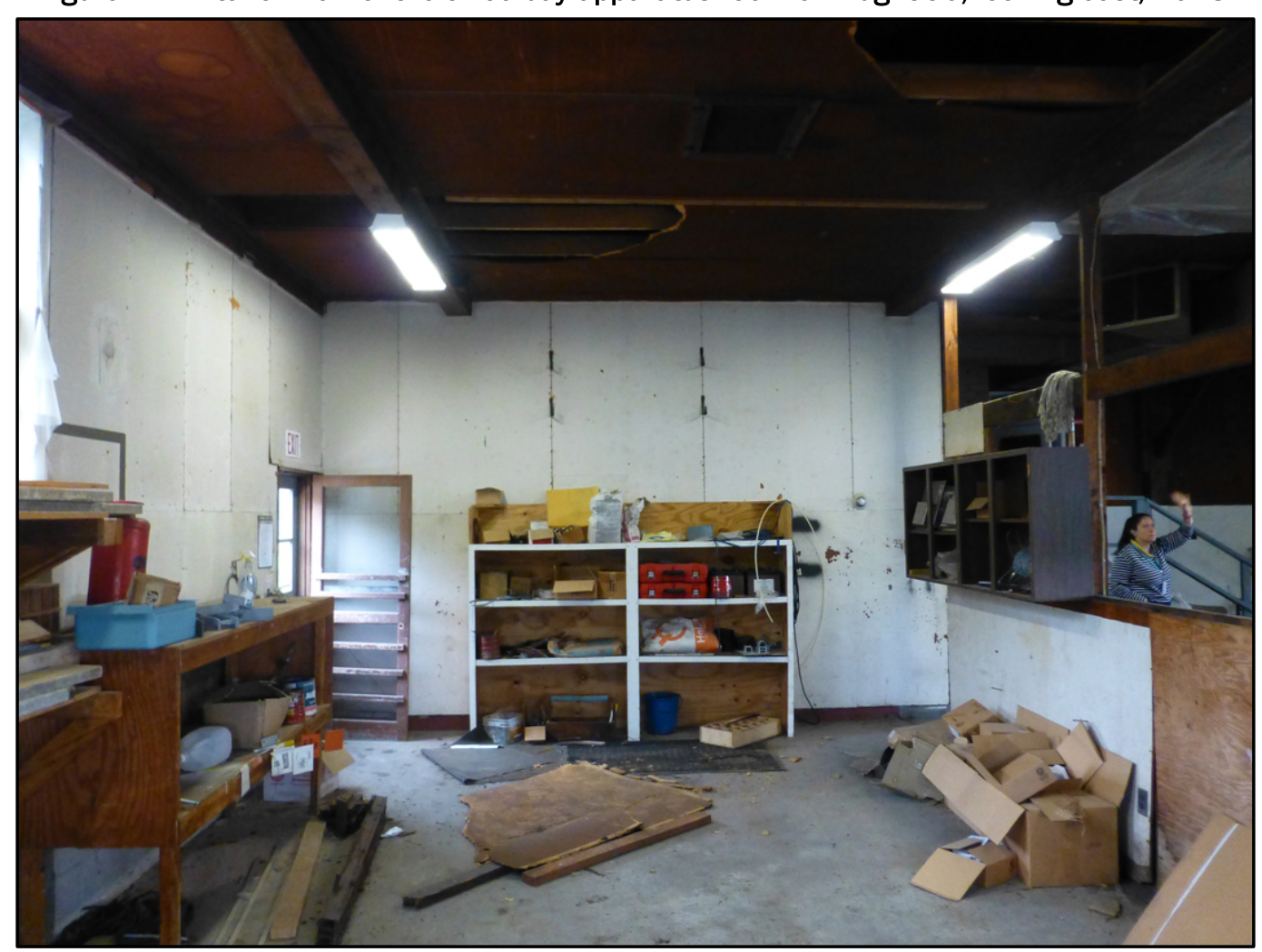

5 The original plans for the alarm room in the 3-bay fire station do not show a zinc sink, enamel sink, or wood cabinetry. All three of these items in the alarm room are of the period of significance and are treated as original to the building. 
Figure 18. Interior view of the three-bay apparatus room of Bldg. 550, looking south, 2018.

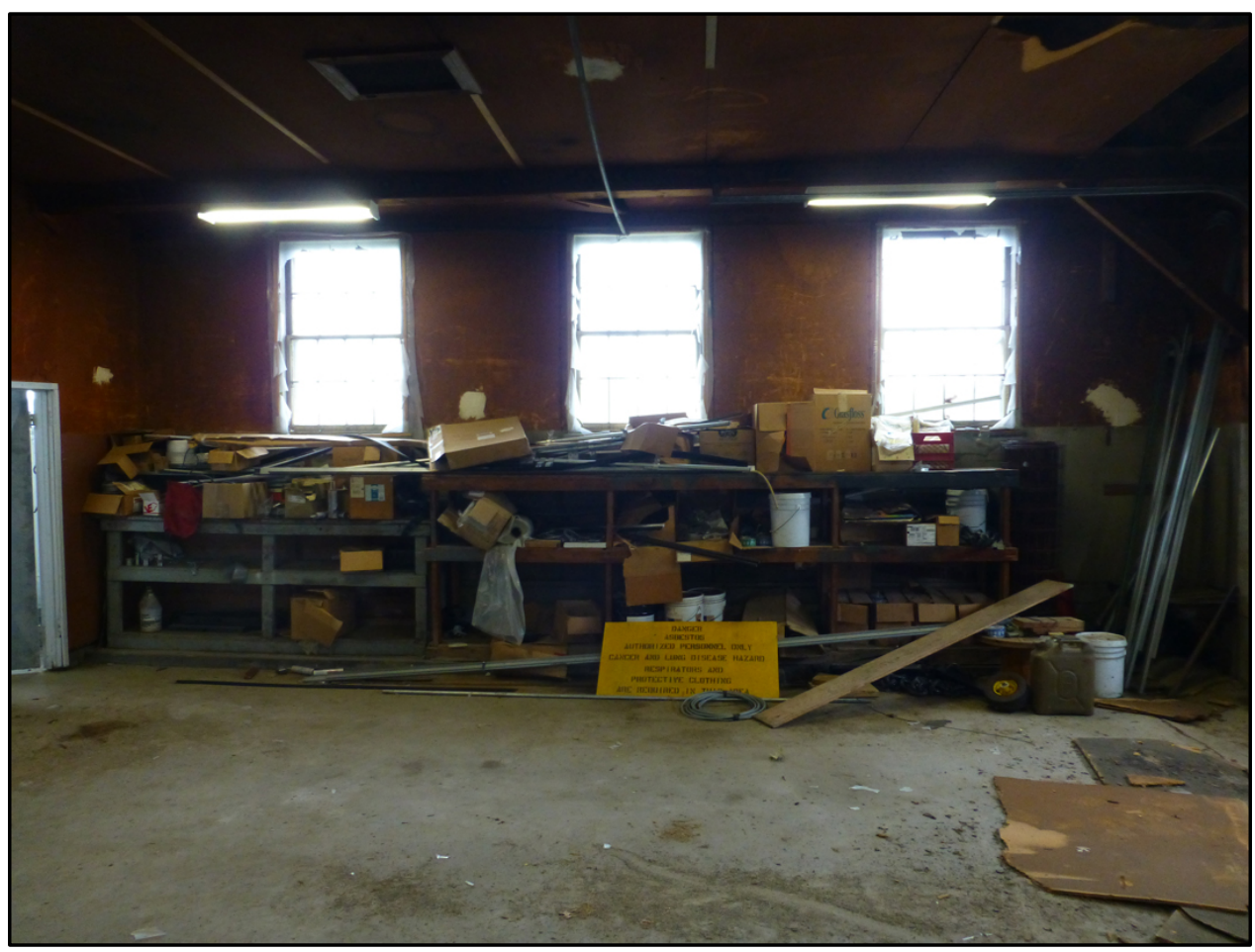

Figure 19. Interior view of the three-bay apparatus room of Bldg. 550, looking west at two of the three replacement overhead doors, 2018.

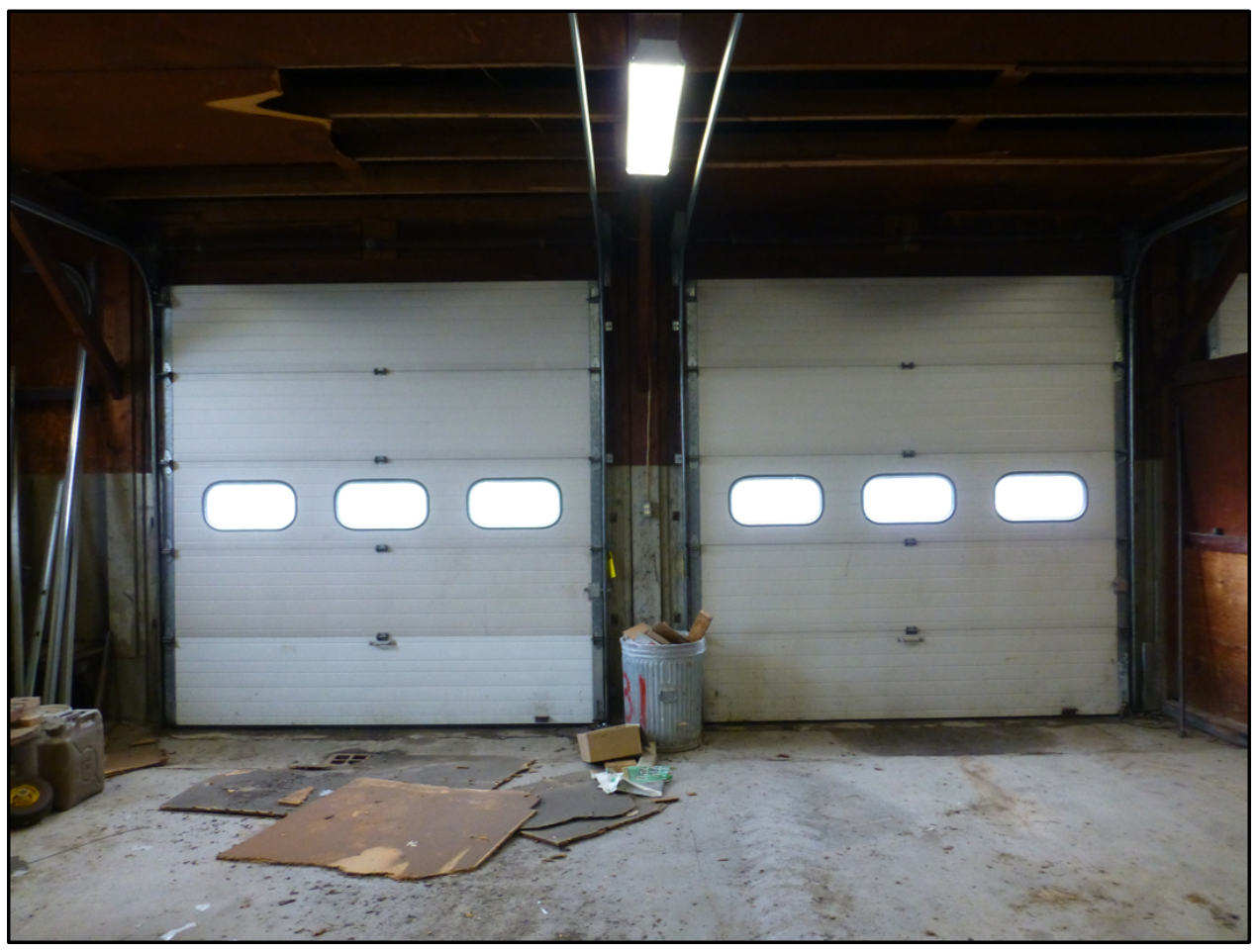


Figure 20. View of the concrete foundation wall of the apparatus room of Bldg. 550, 2018.

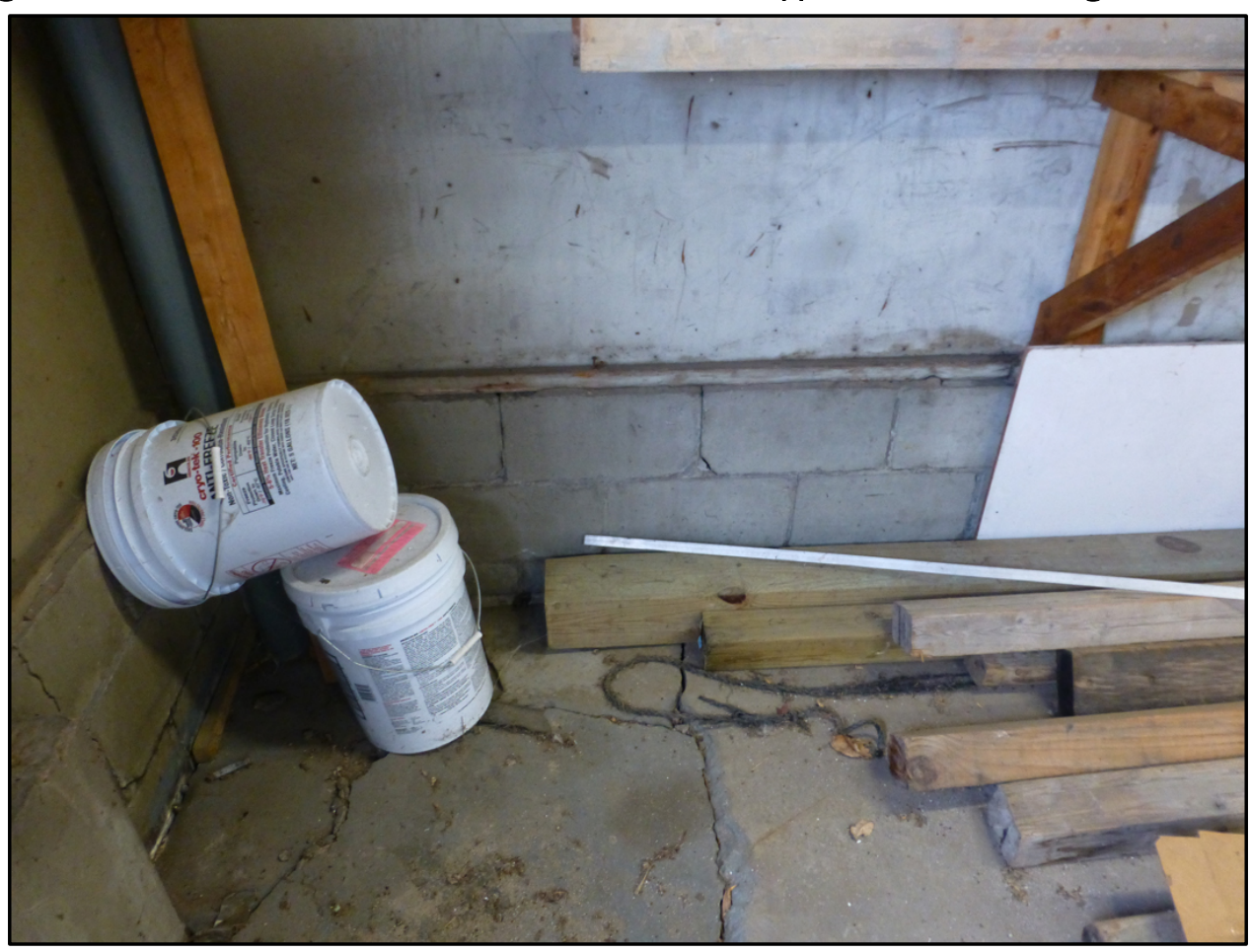

Figure 21. Original water fountain located right outside the work room of Bldg. 550, 2018.

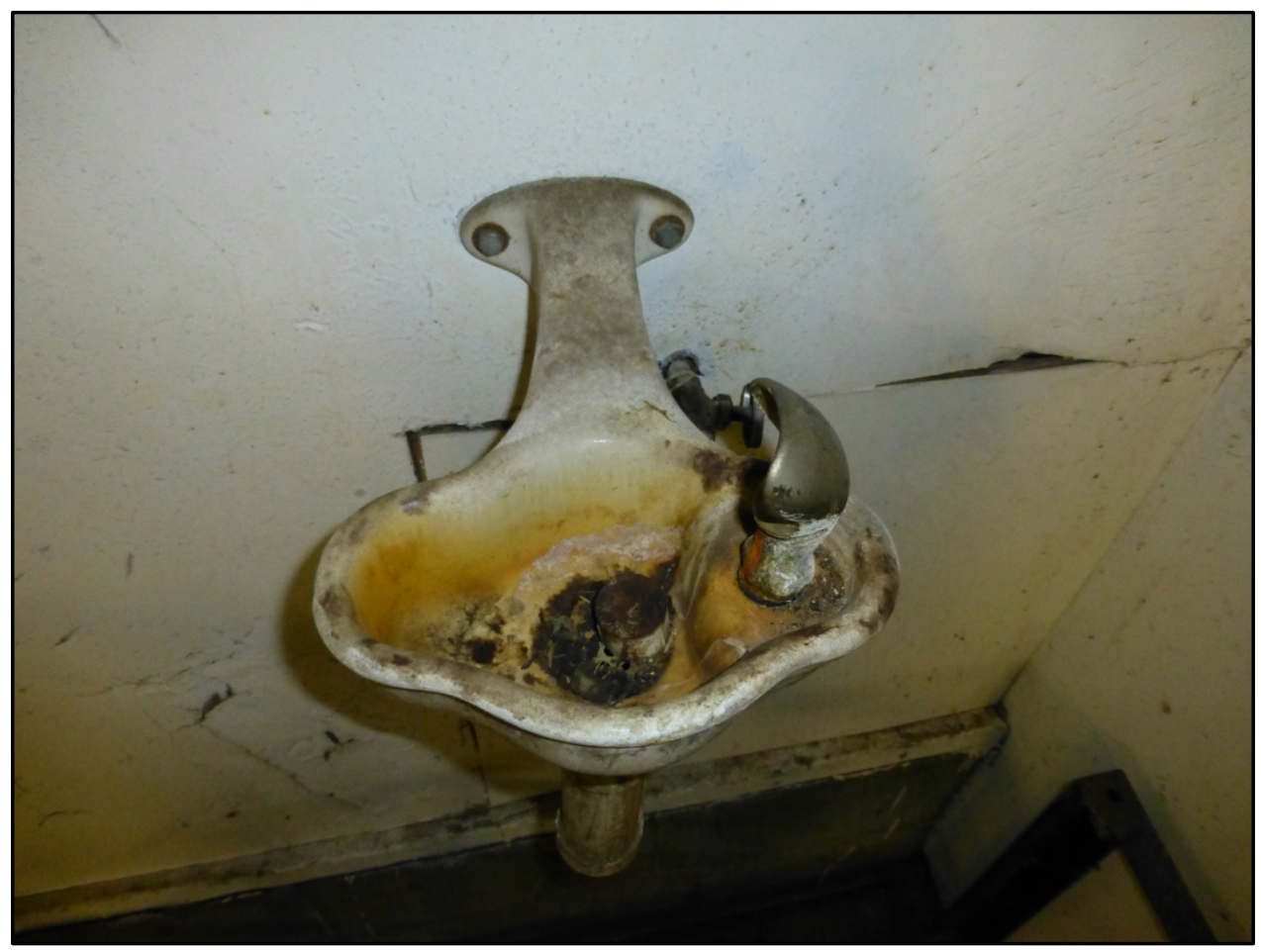


Figure 22. Original enamel mop sink located in the work room of the three-bay apparatus room of Bldg. 550, 2018.

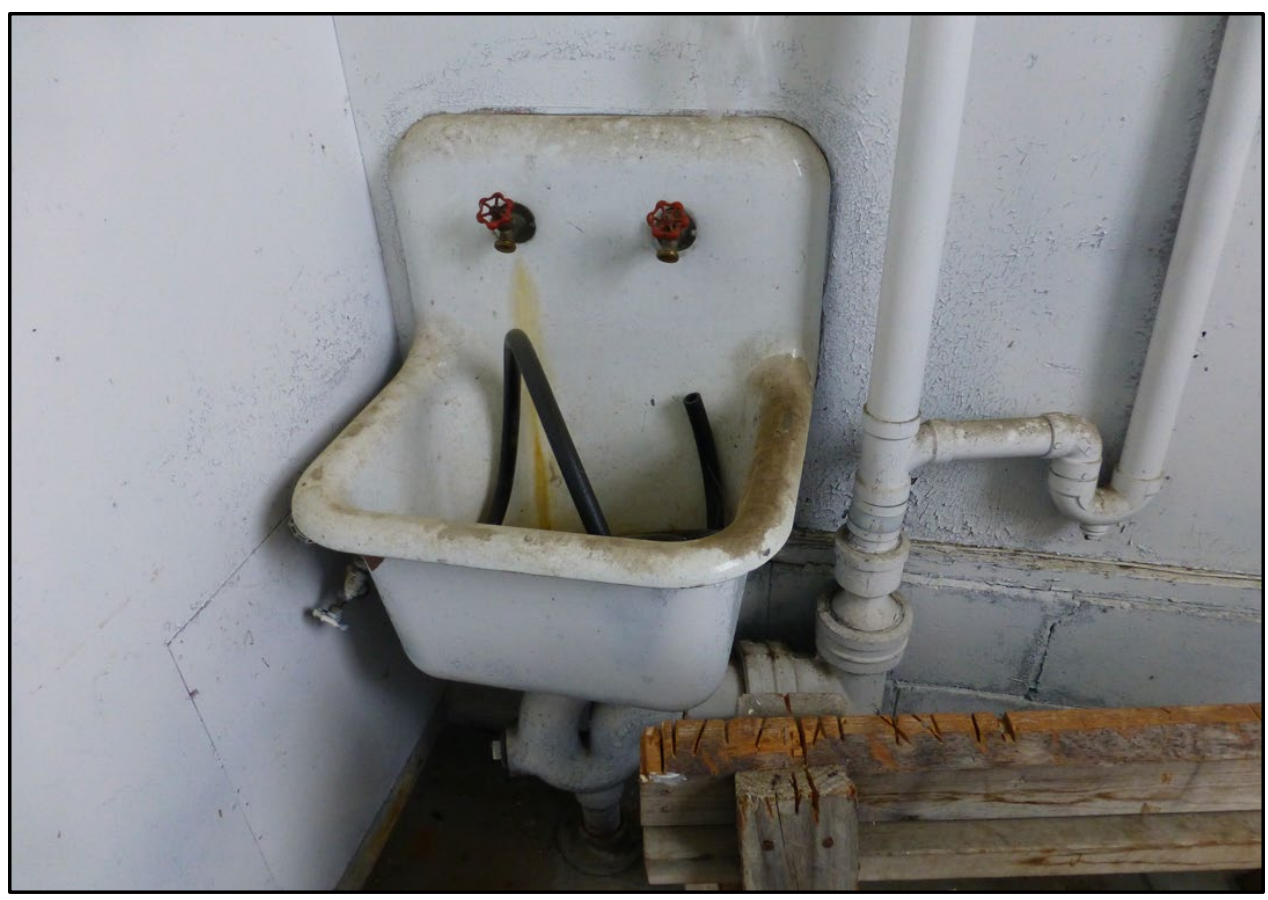

Figure 23. Metal sink washing area located in the original alarm room of the three-bay apparatus room in Bldg. 550, 2018. ${ }^{6}$

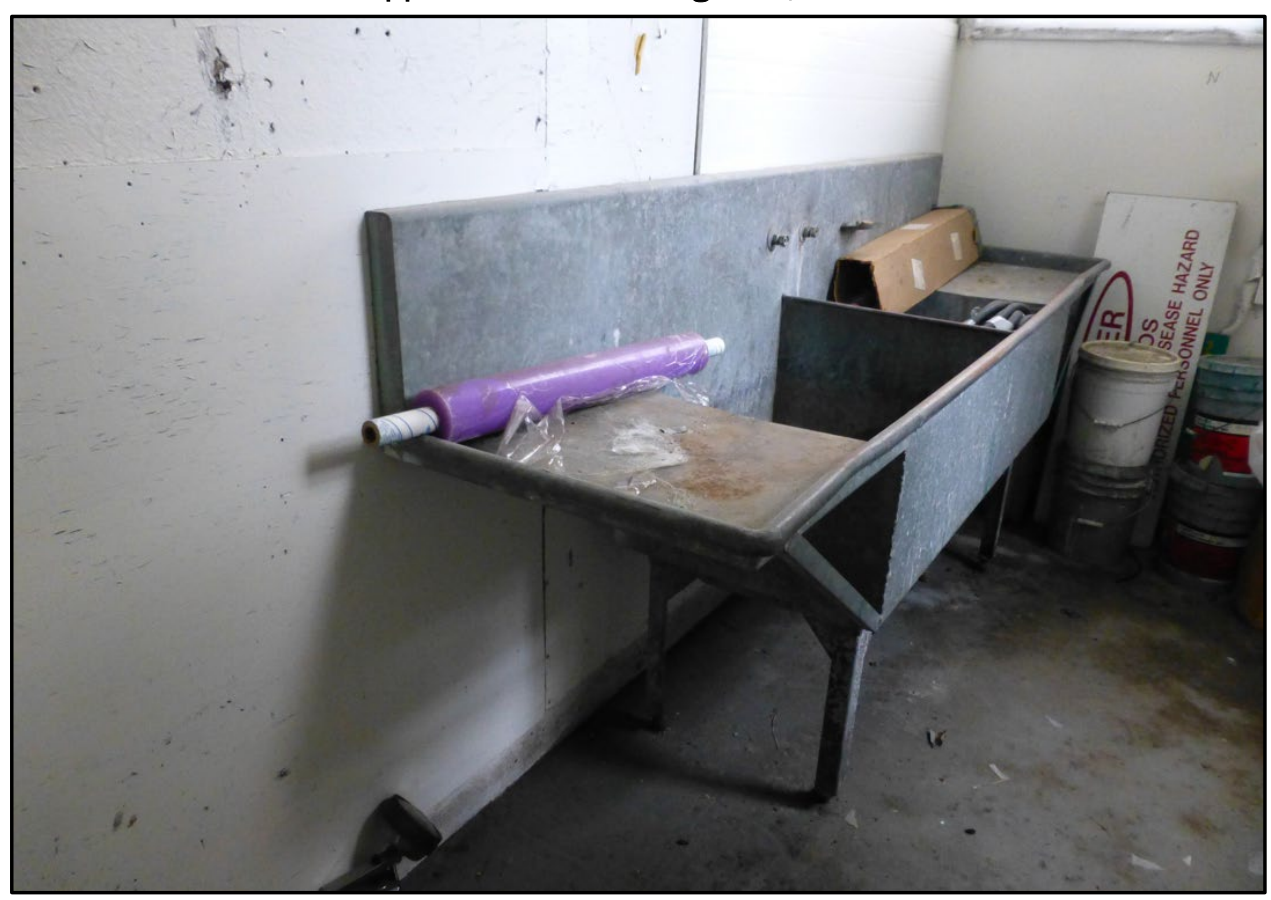

6 See footnote 5 . 
Figure 24. Original cabinetry in the original alarm room of the three-bay apparatus room of BIdg. 550, 2018. ${ }^{7}$

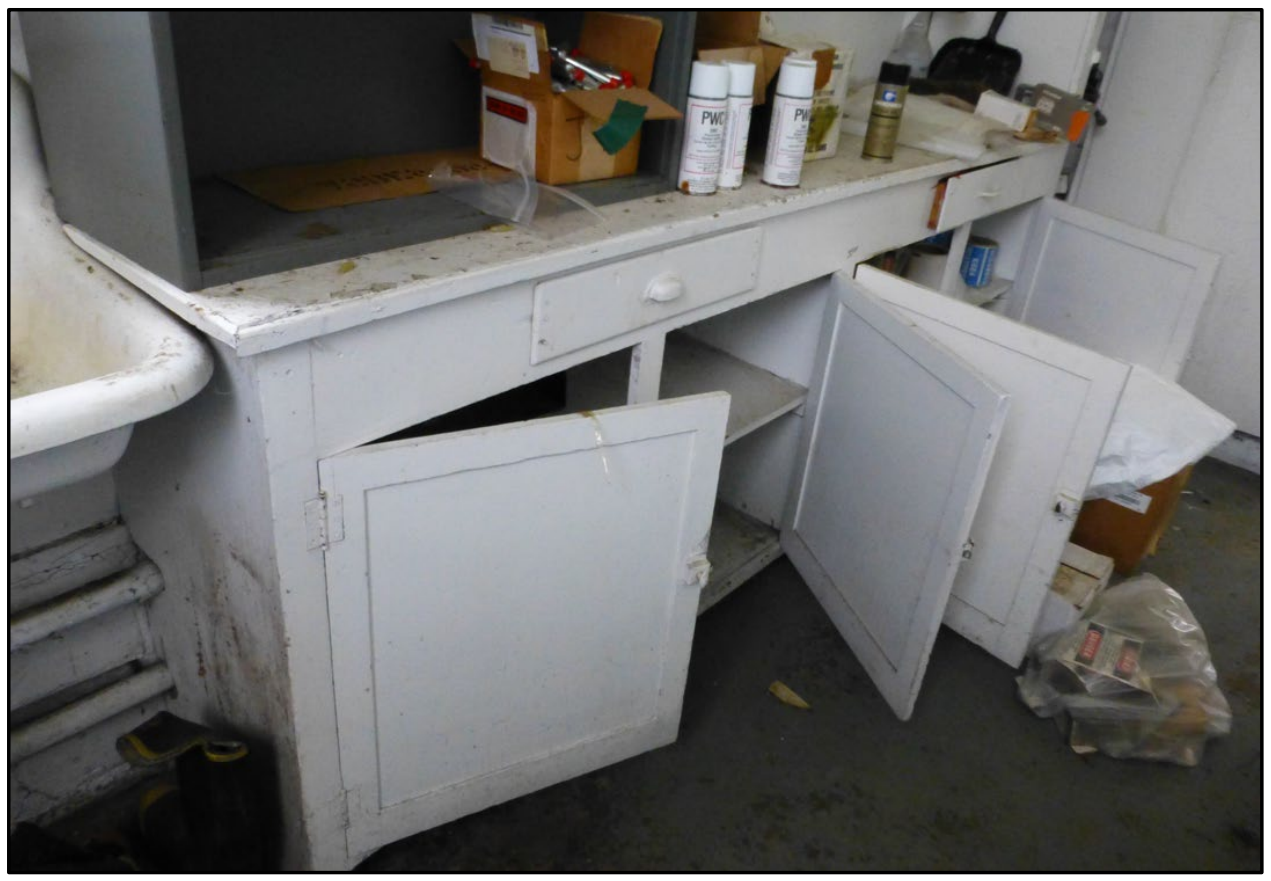

Figure 25. Original enamel sink in the original alarm room of the three-bay apparatus room of Bldg. 550, 2018. ${ }^{8}$

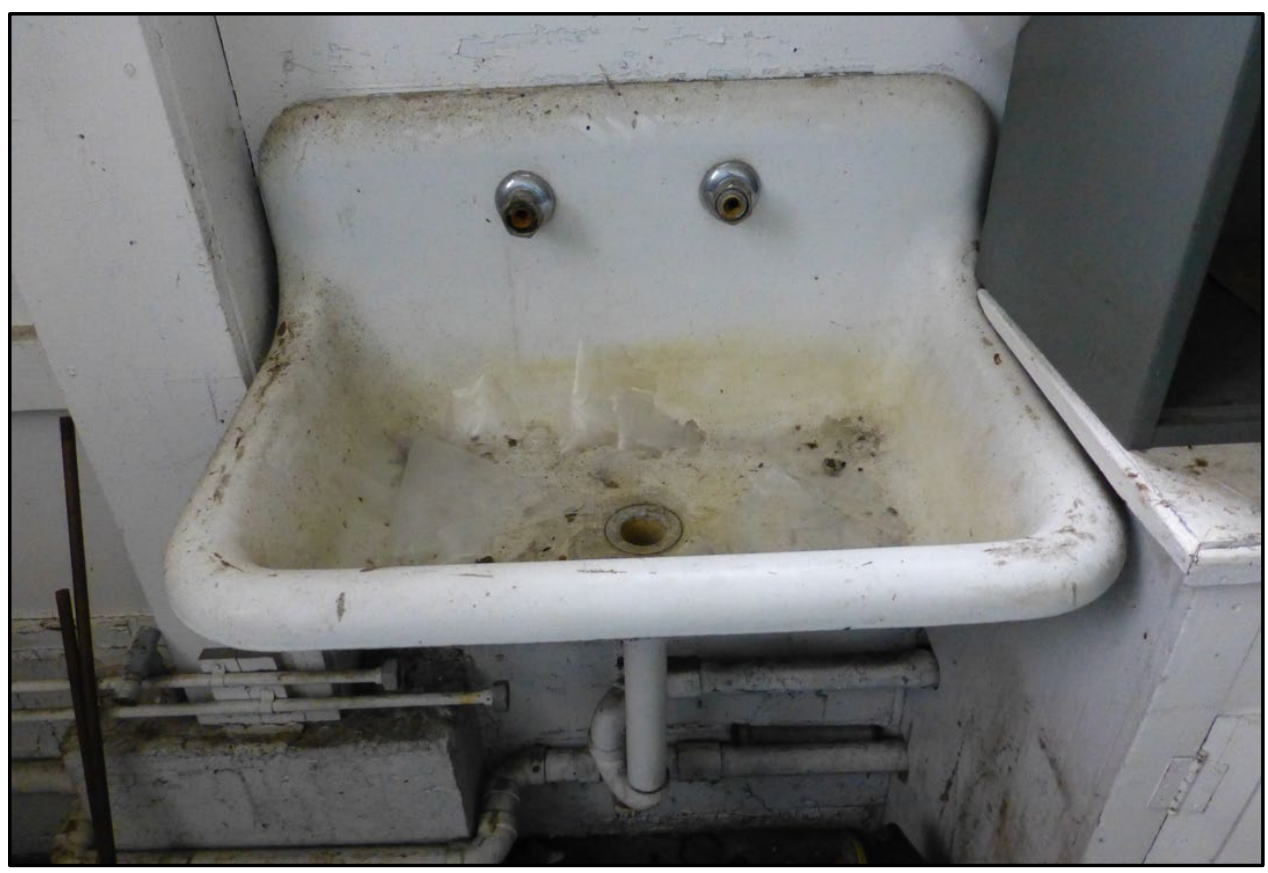

\footnotetext{
7 See footnote 5.

8 See footnote 5 .
} 
Figure 26. Looking east from the three-bay apparatus room of BIdg. 550 towards the elevated living area of the building, 2018.

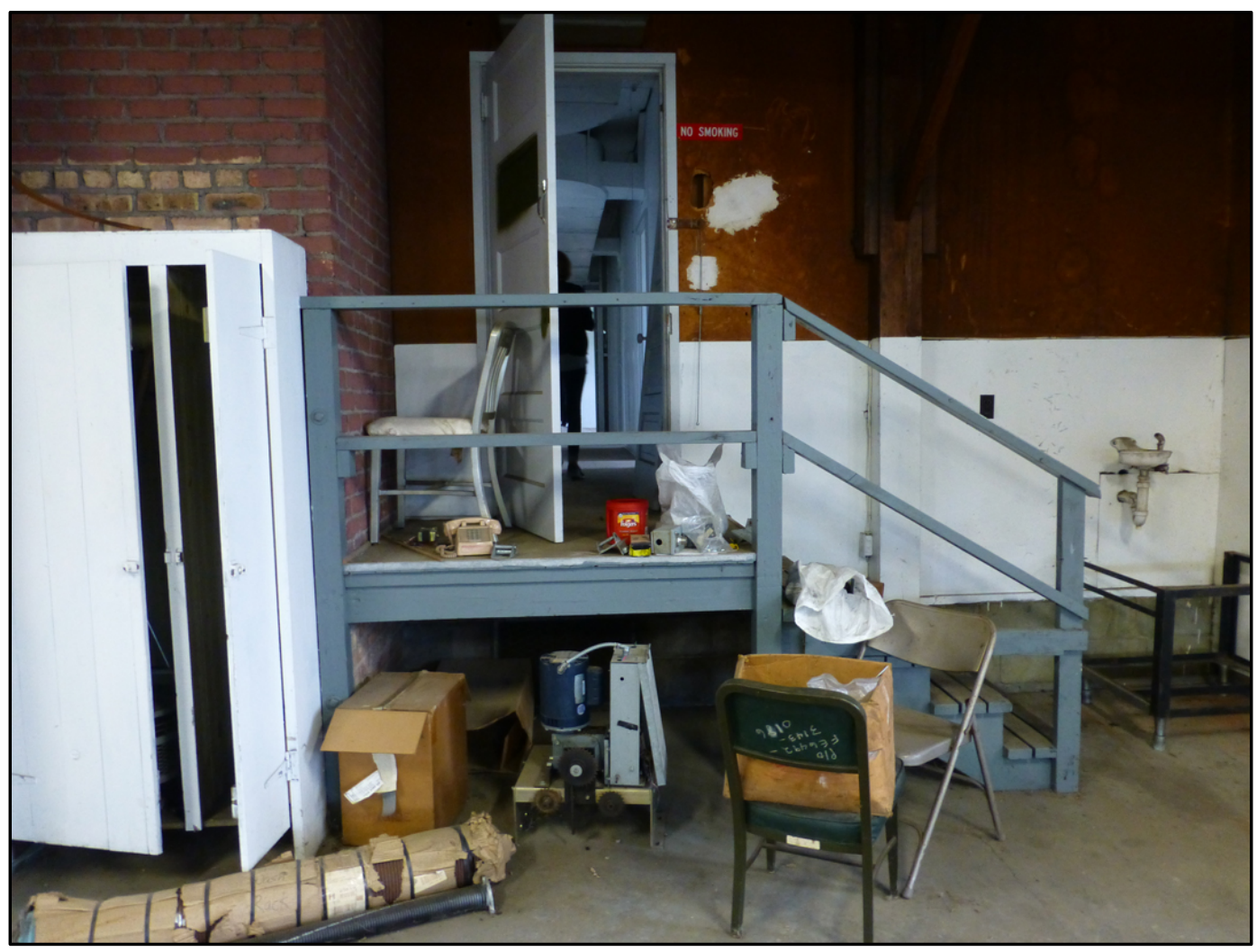

A central corridor divides the west end of the living area (see Figure 27). A heater room and small open room (officer room) is located to the north, while a toilet room and small room (non-commissioned officer [NCO] room) are located to the south of the corridor. The toilet room's original fixtures are mostly intact, such as the concrete floor, wood slat walls, shower stall, toilets, urinal, sinks, mirrors, light fixtures, and shelves (see Figures 28 through 33). A new door opening has been cut into the east wall of the toilet room to adjoin it to the adjacent small room (see Figure 31). The walls in the NCO room are open studs (see Figure 34). 
Figure 27. Looking east down the central corridor of the living area of Bldg. 550, 2018.

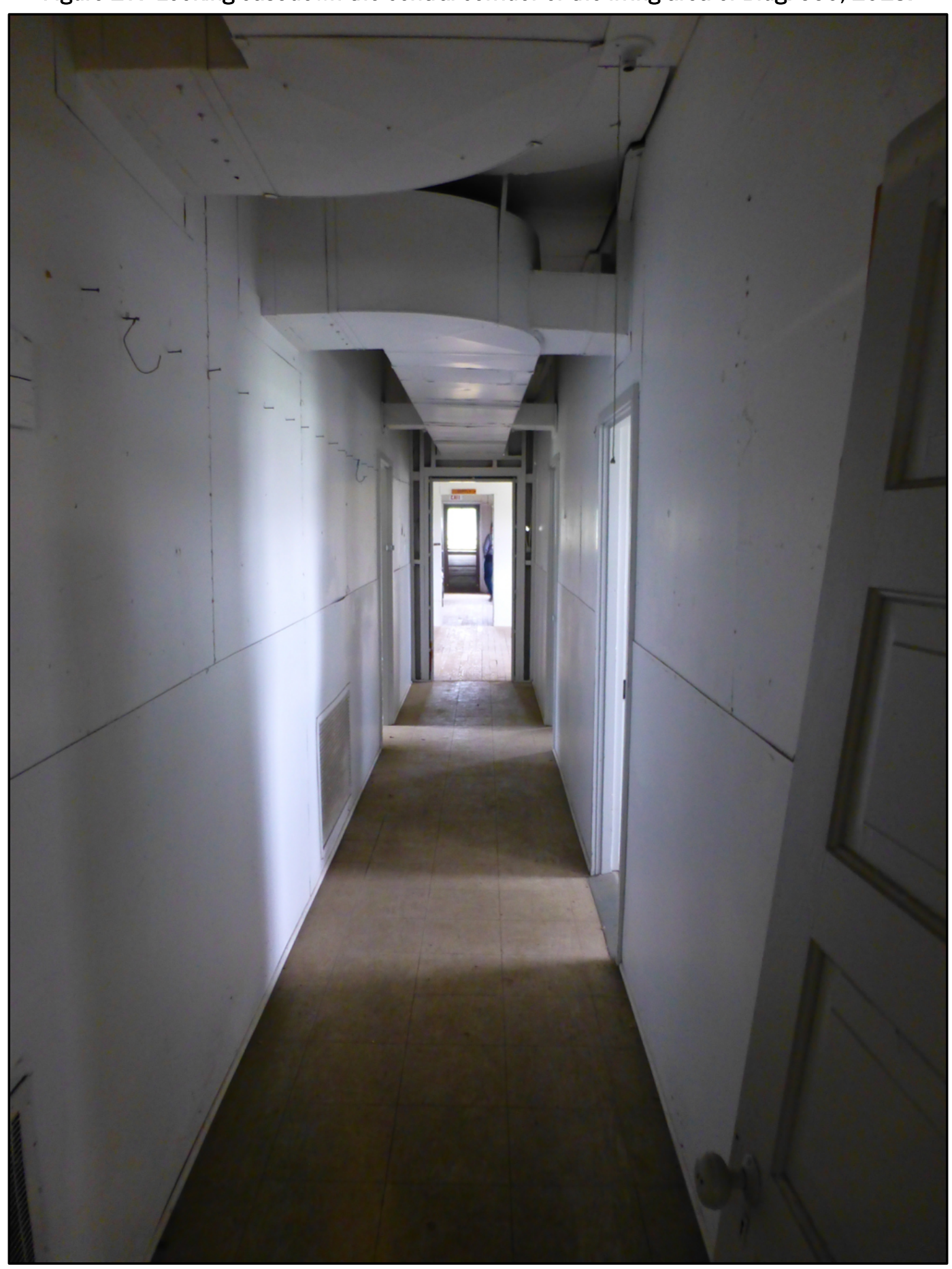


Figure 28. Looking west at latrine area in Bldg. 550 with original wood plank walls, exposed ceiling, concrete floor, and urinal, 2018.

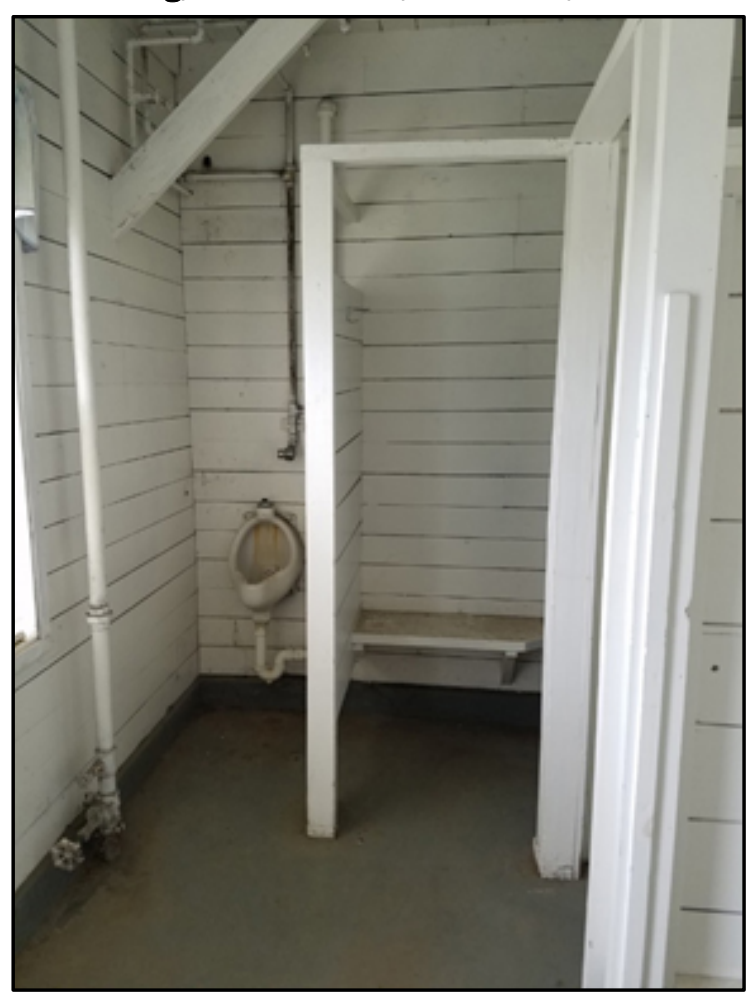

Figure 29. Original toilet with original wood stall walls in the latrine of Bldg. 550, 2018.

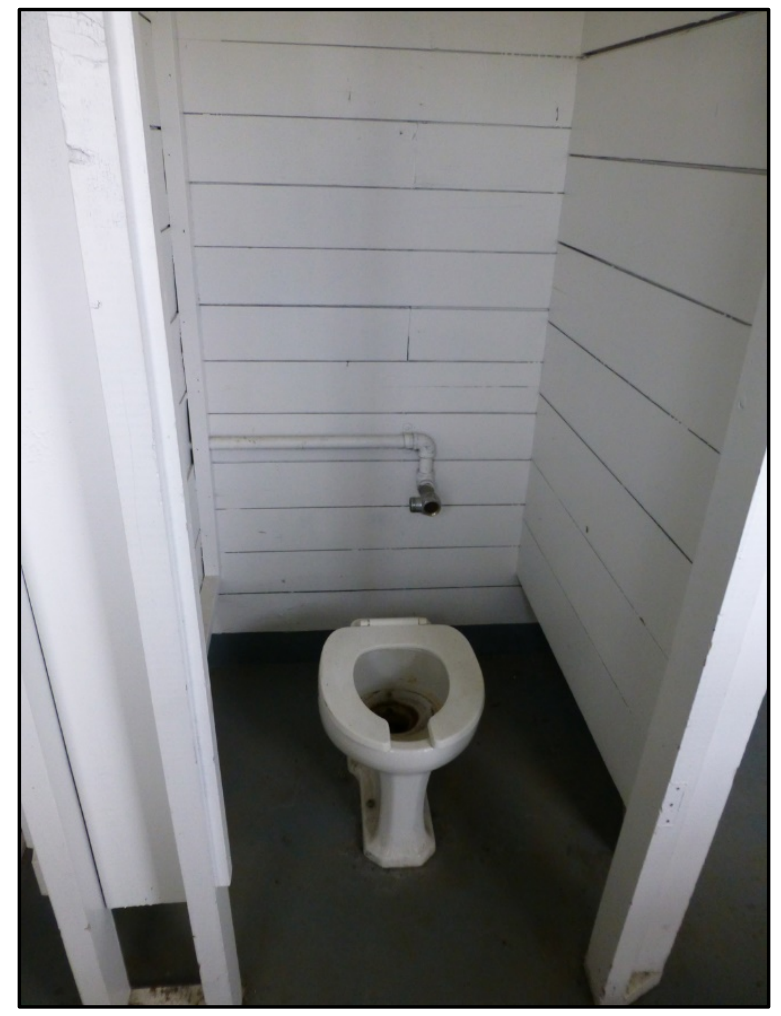


Figure 30. Original toilet paper holder in Bldg. 550, 2018.

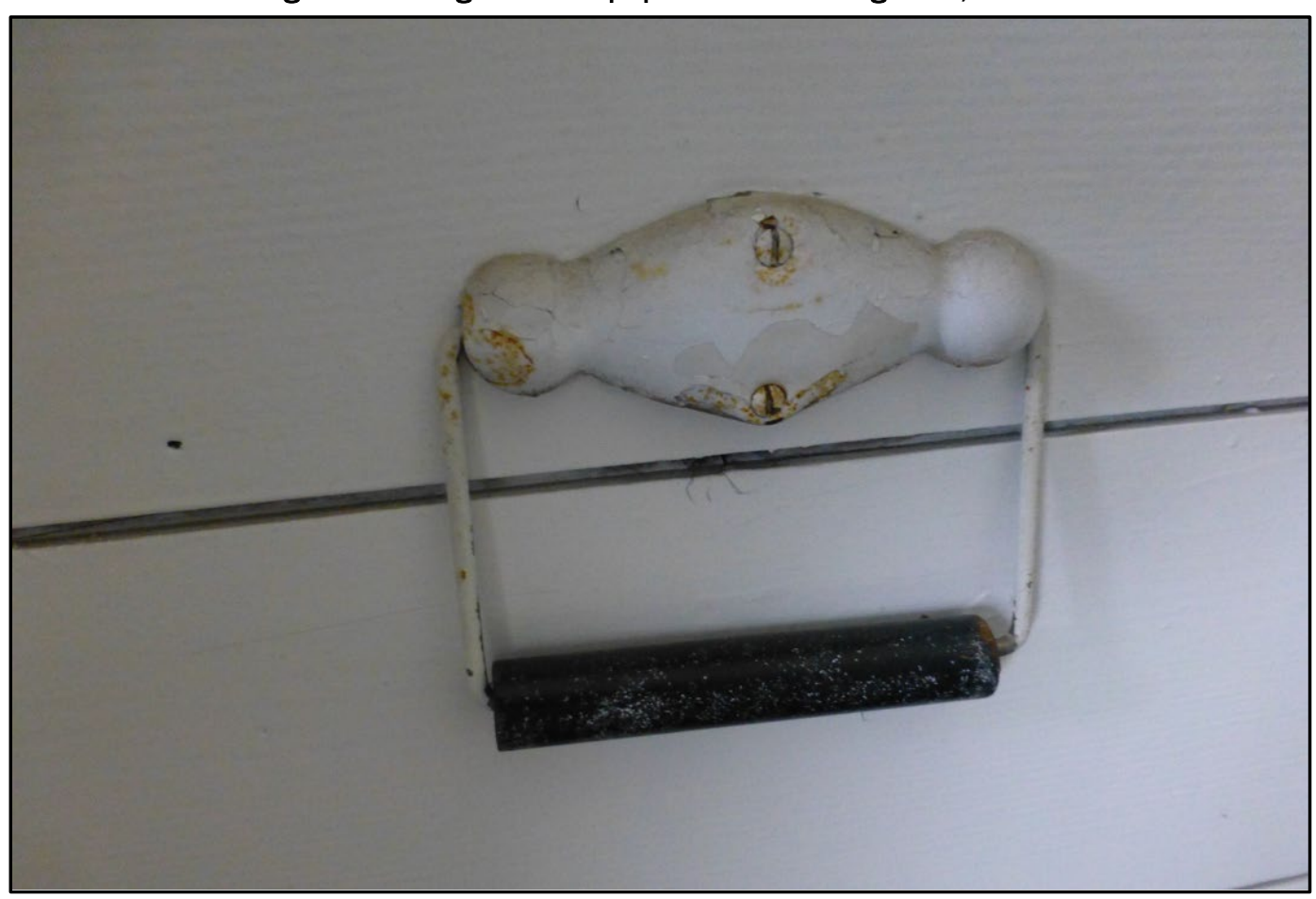

Figure 31. Original sinks, wood plank walls, mirrors, and shelves in Bldg. 550, 2018.

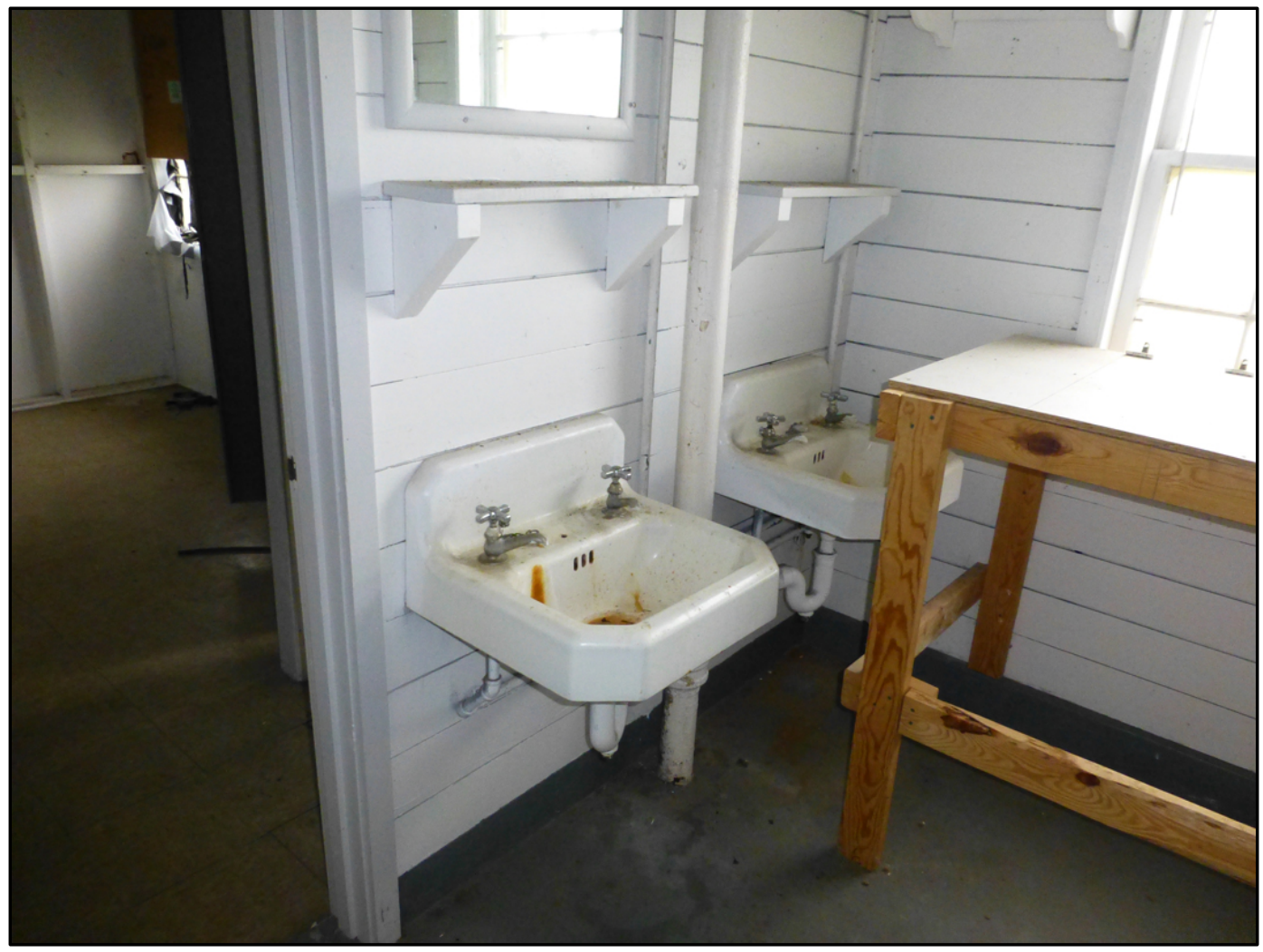


Figure 32. Original light fixture above latrine sinks in Bldg. 550, 2018.

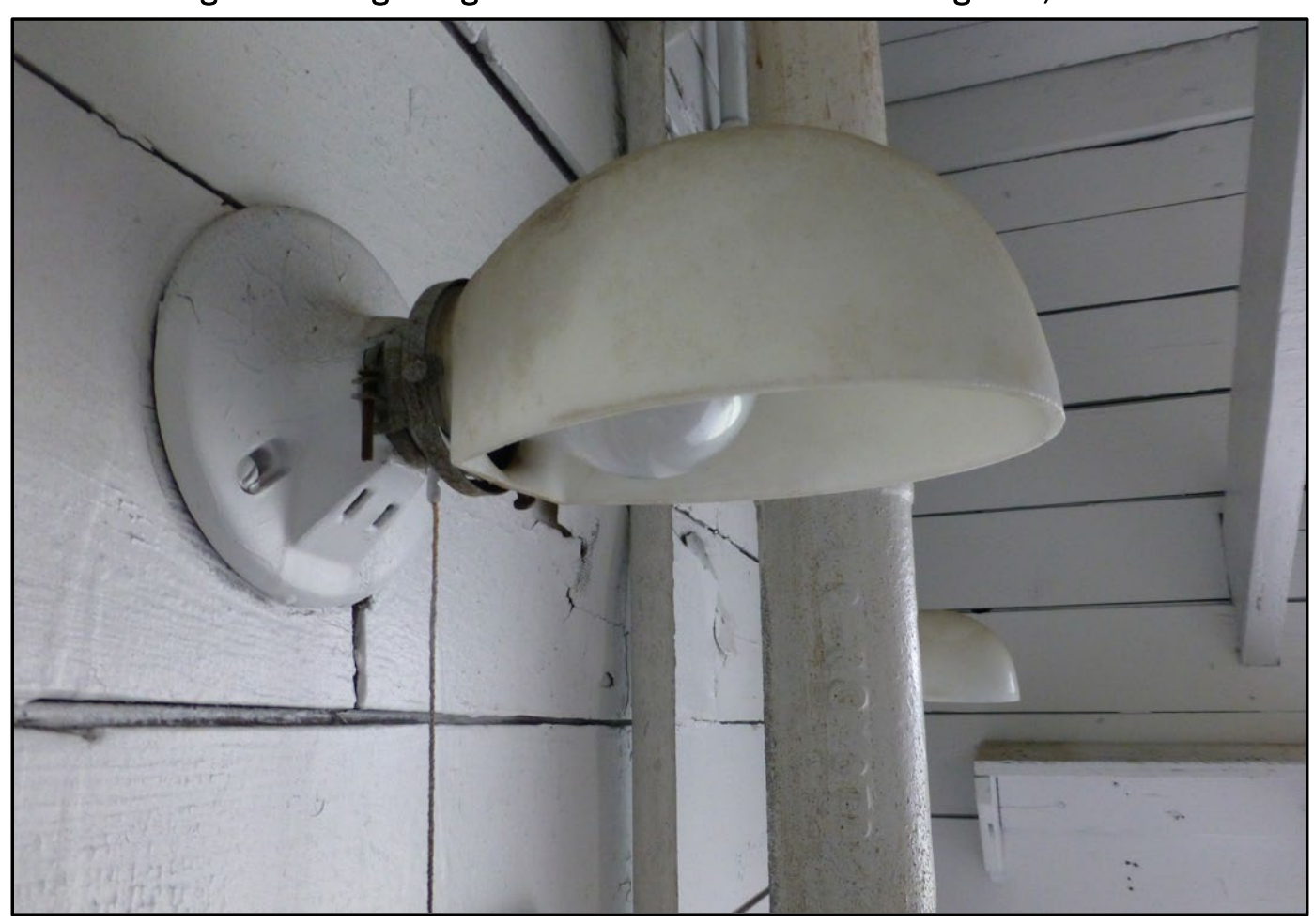

Figure 33. Original shower stall in Bldg. 550 with wood plank floor, 2018.

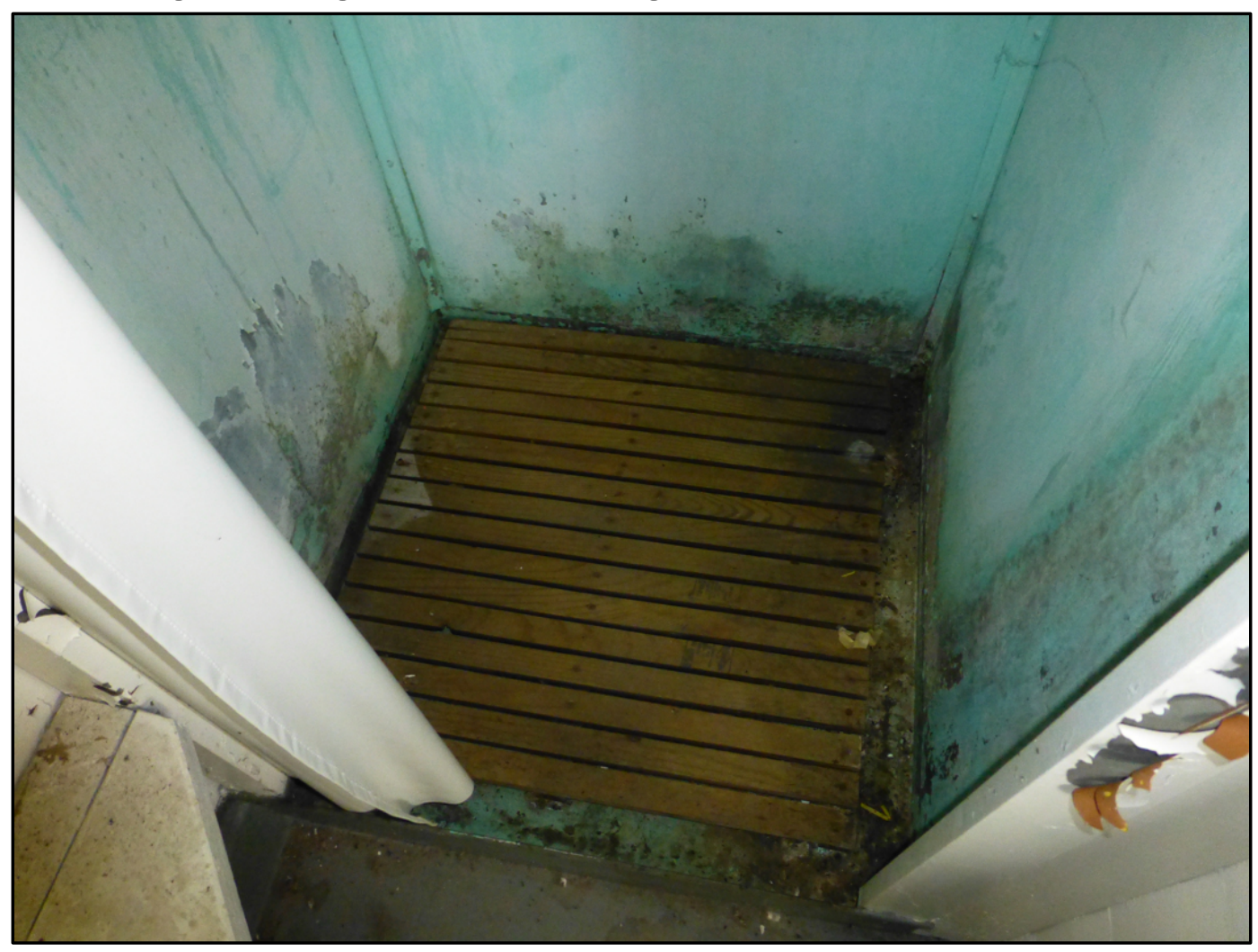


Figure 34. Looking east at wall in small room east of latrine in Bldg. 550, 2018.

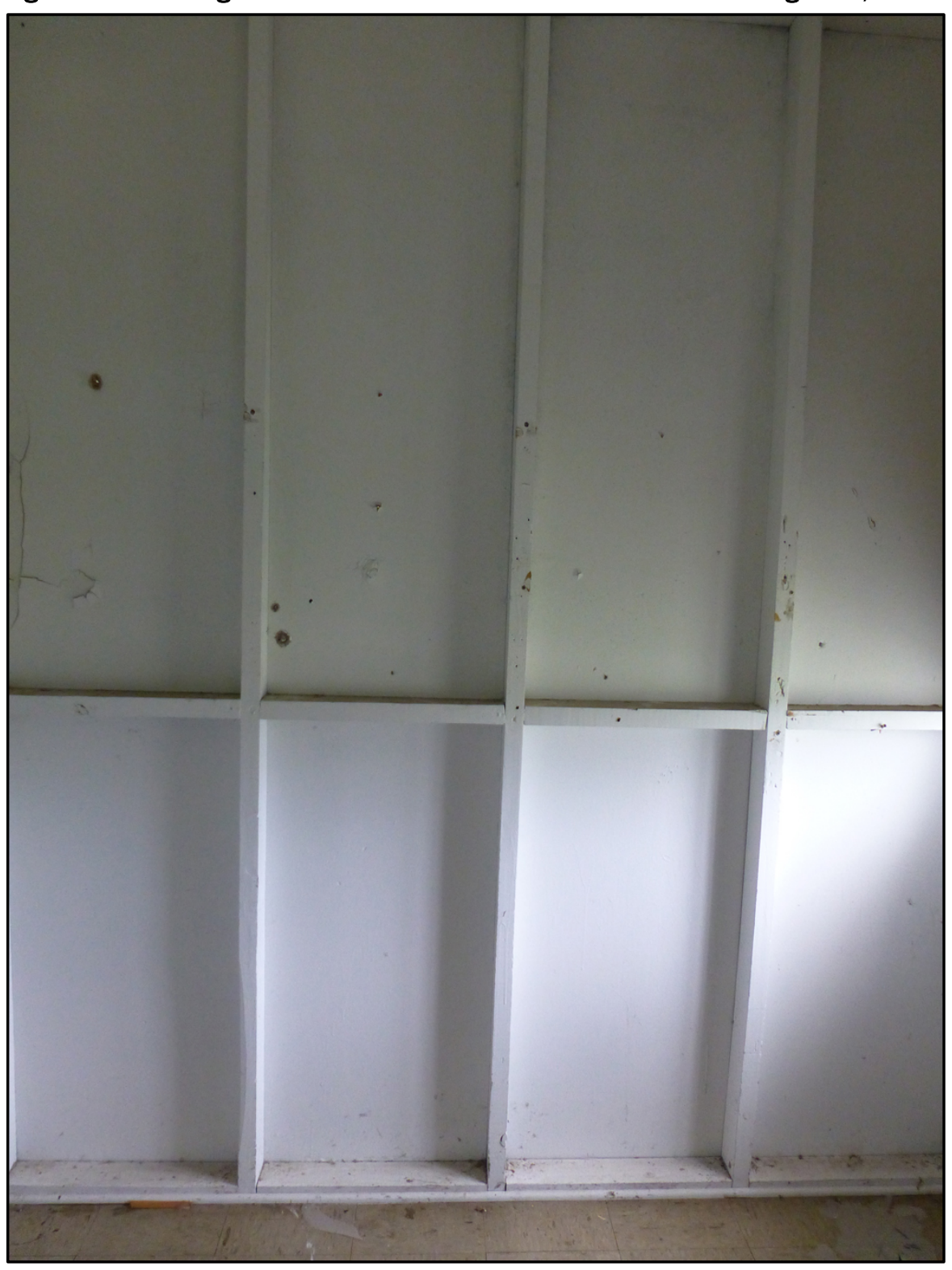

At the east end of the corridor is a large open space that was the former squad room and it has been slightly modified with a newer wood frame partition wall (see Figure 35). The open room has original wood floors and walls and ceiling clad with original wall board (fiberboard) and wood battens (see Figures 36 and 37). Two sets of original wood built-in closet units are located along the east wall of the room ${ }^{9}$ (see Figure 38 ).

\footnotetext{
9 The original plans for the squad room in the 3-bay fire station do not show lockers. The lockers in the squad room are of the period and are treated as original to the building.
} 
Figure 35. Looking west at non-original wall dividing the squad room in half in Bldg. 550, 2018.

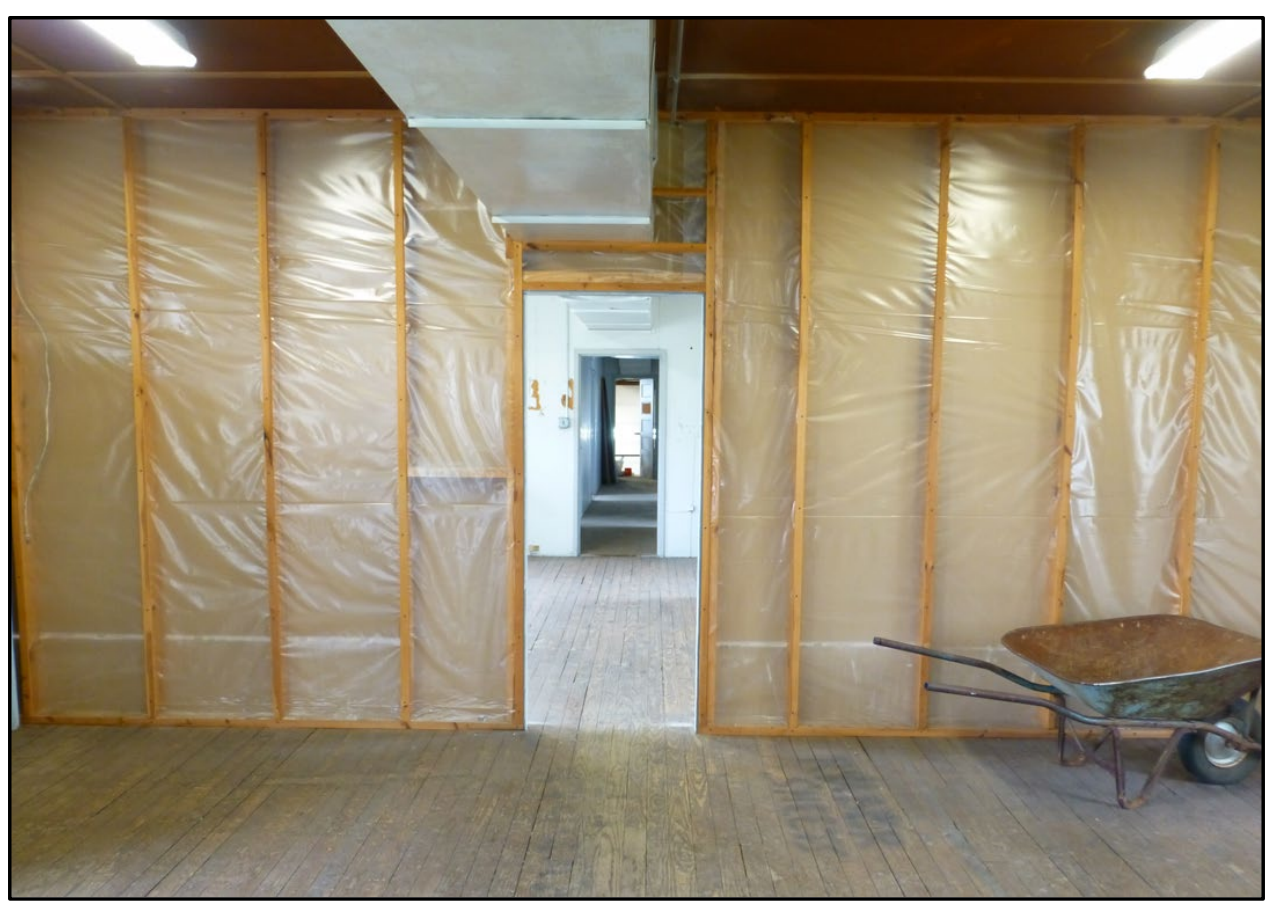

Figure 36. Looking down at the original wood floor in the squad room in Bldg. 550, 2018.

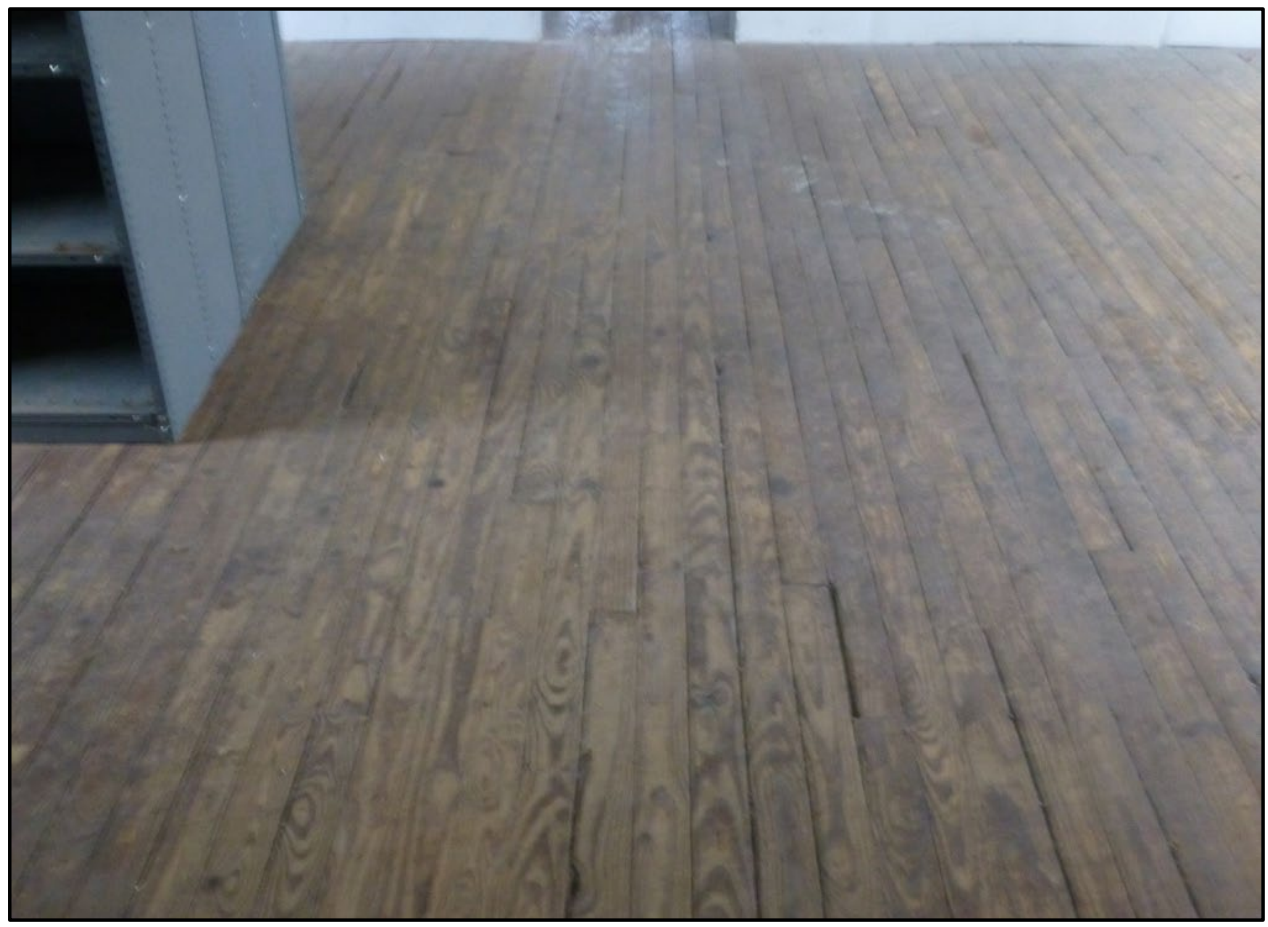


Figure 37. Looking up at the original wall board (fiber board) and batten ceiling with original ceiling vent in the squad room in BIdg. 550, 2018.

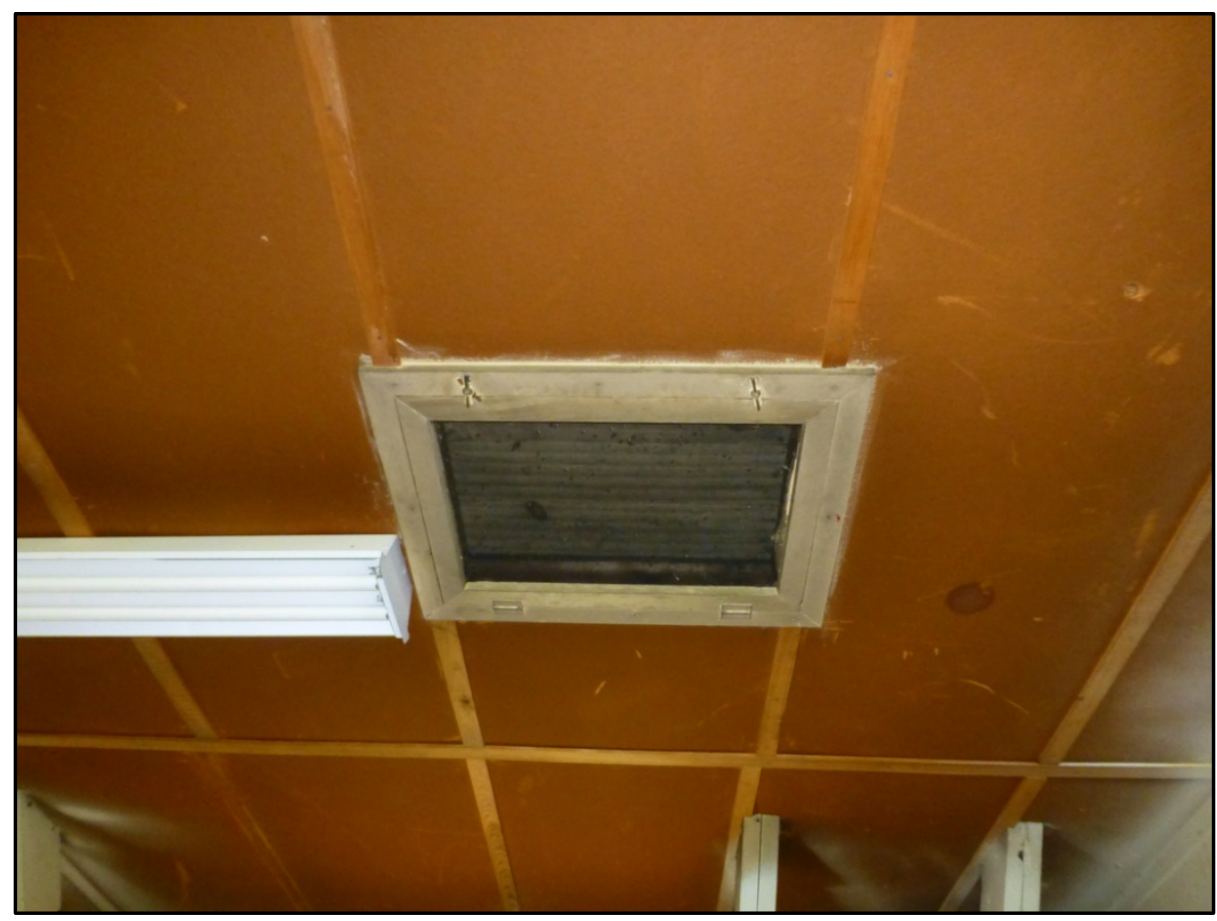

Figure 38. Looking west at the original wood built-in closets in the squad room in Bldg. 550, 2018. ${ }^{10}$

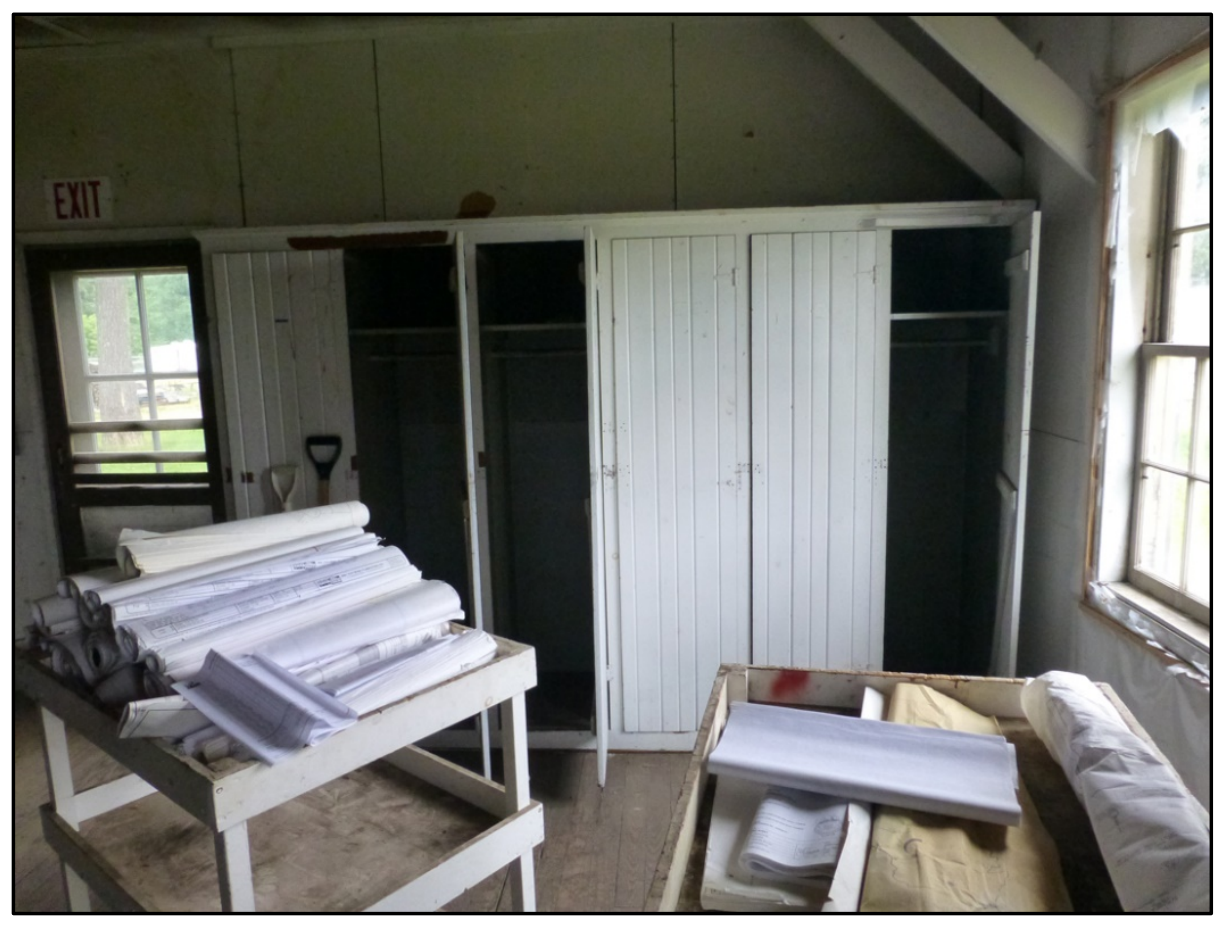

10 See footnote 9 . 
The interior doors are original wood panel doors (see Figure 39). There are original wood screen doors leading out to the exterior of the building (see Figure 40). The original suspended mechanical systems and duct work is intact in the open room and central corridor (see Figures 41 and 42). The original ceiling vents are intact throughout the living area of Bldg. 550 (Figure 43).

Figure 39. Looking west at an original wood five-panel door in the latrine in BIdg. 550, 2018.

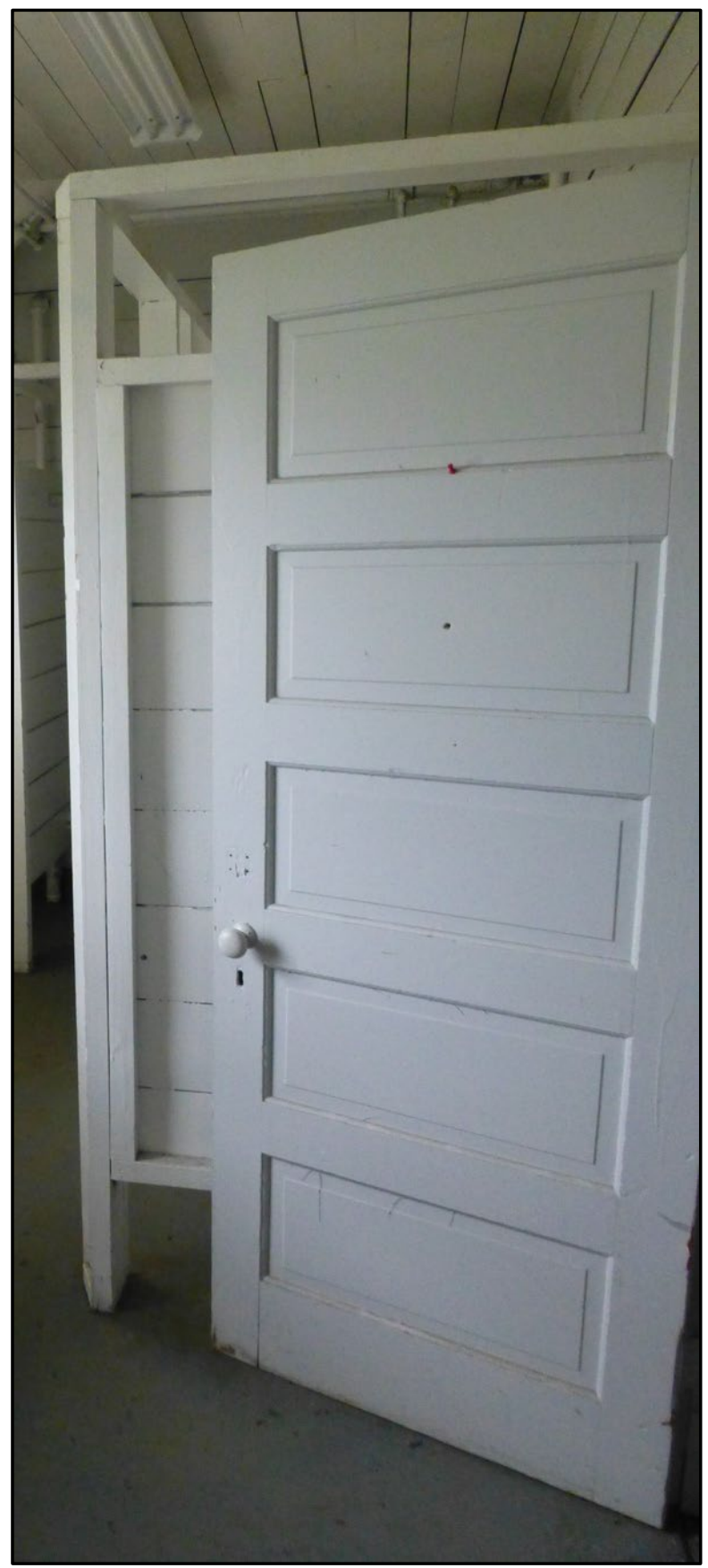


Figure 40. Looking east at an original wooden screen door in Bldg. 550, 2018.

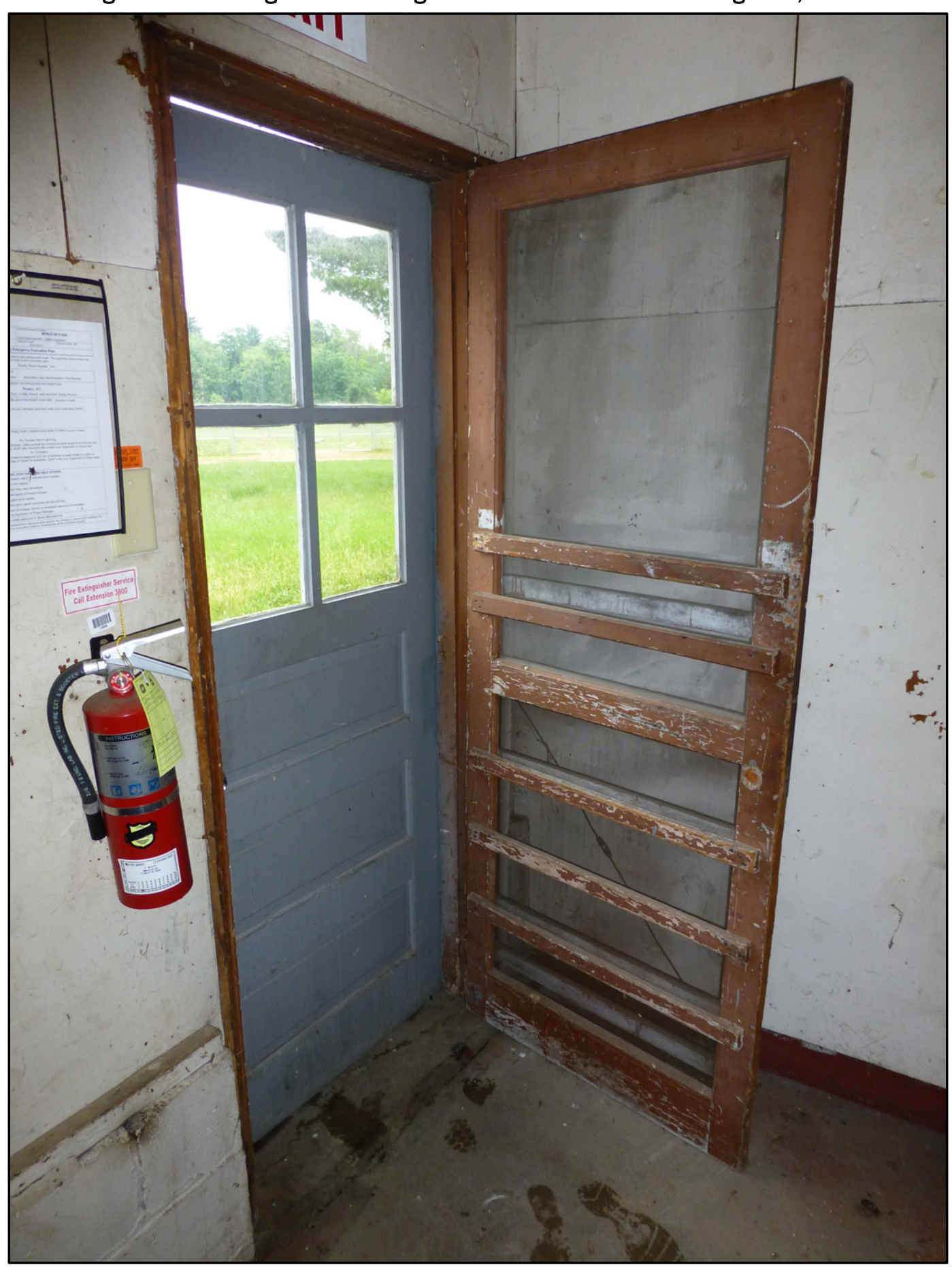


Figure 41. Looking up at original duct work in the central hallway, 2018.

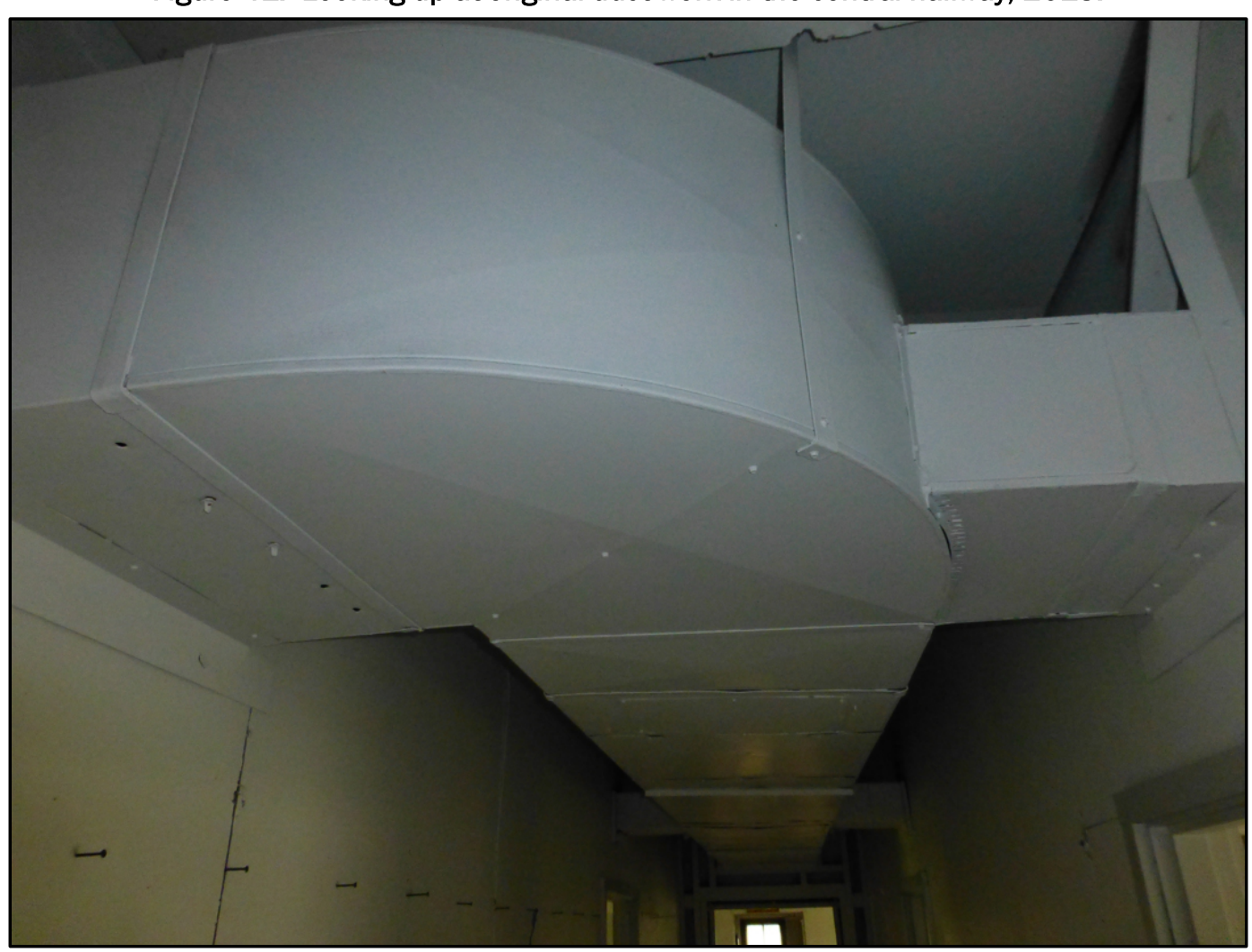

Figure 42. Looking up at original duct work in the squad room, 2018.

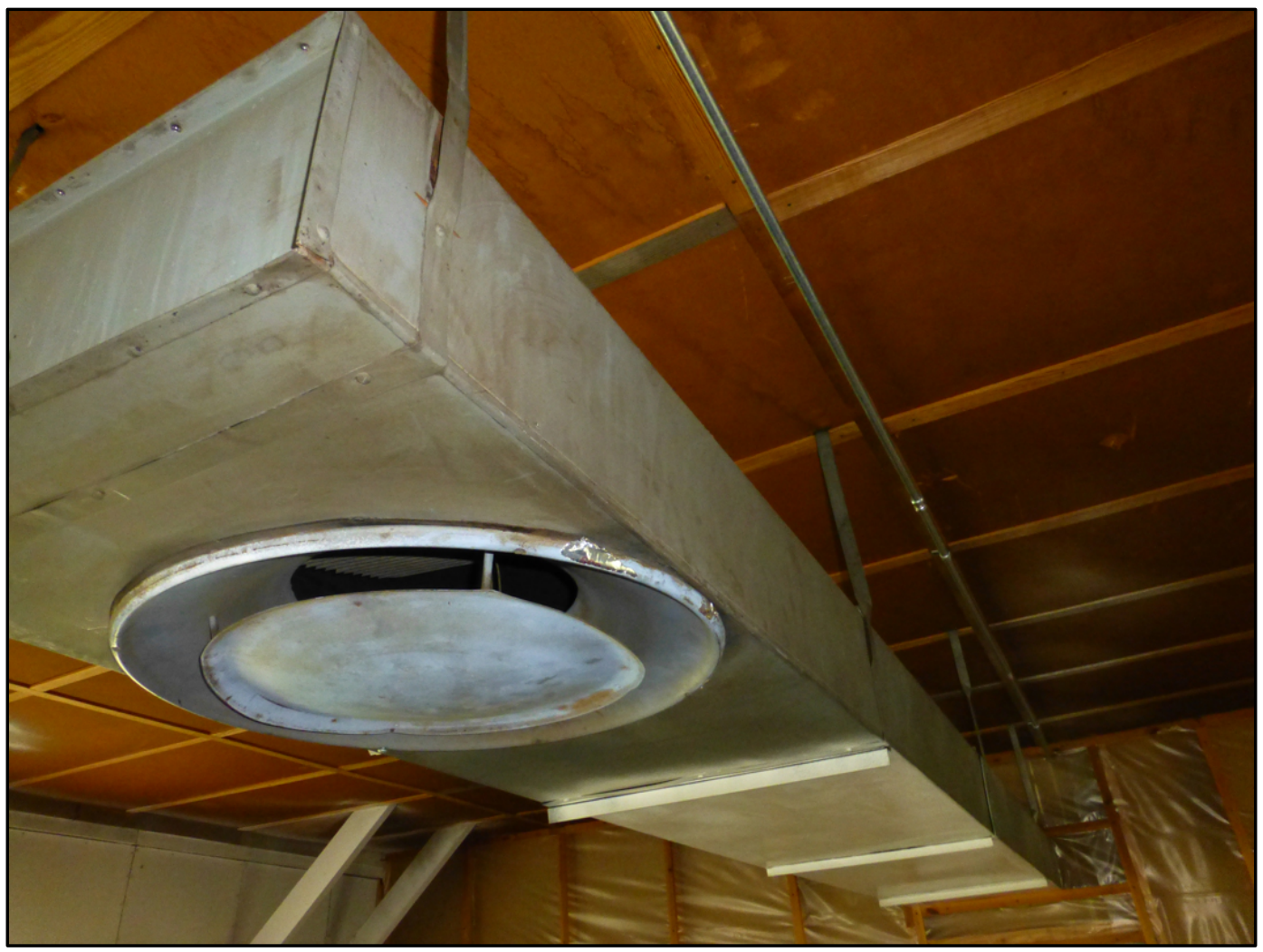


Figure 43. Looking up at original ceiling vent in the squad room, 2018.

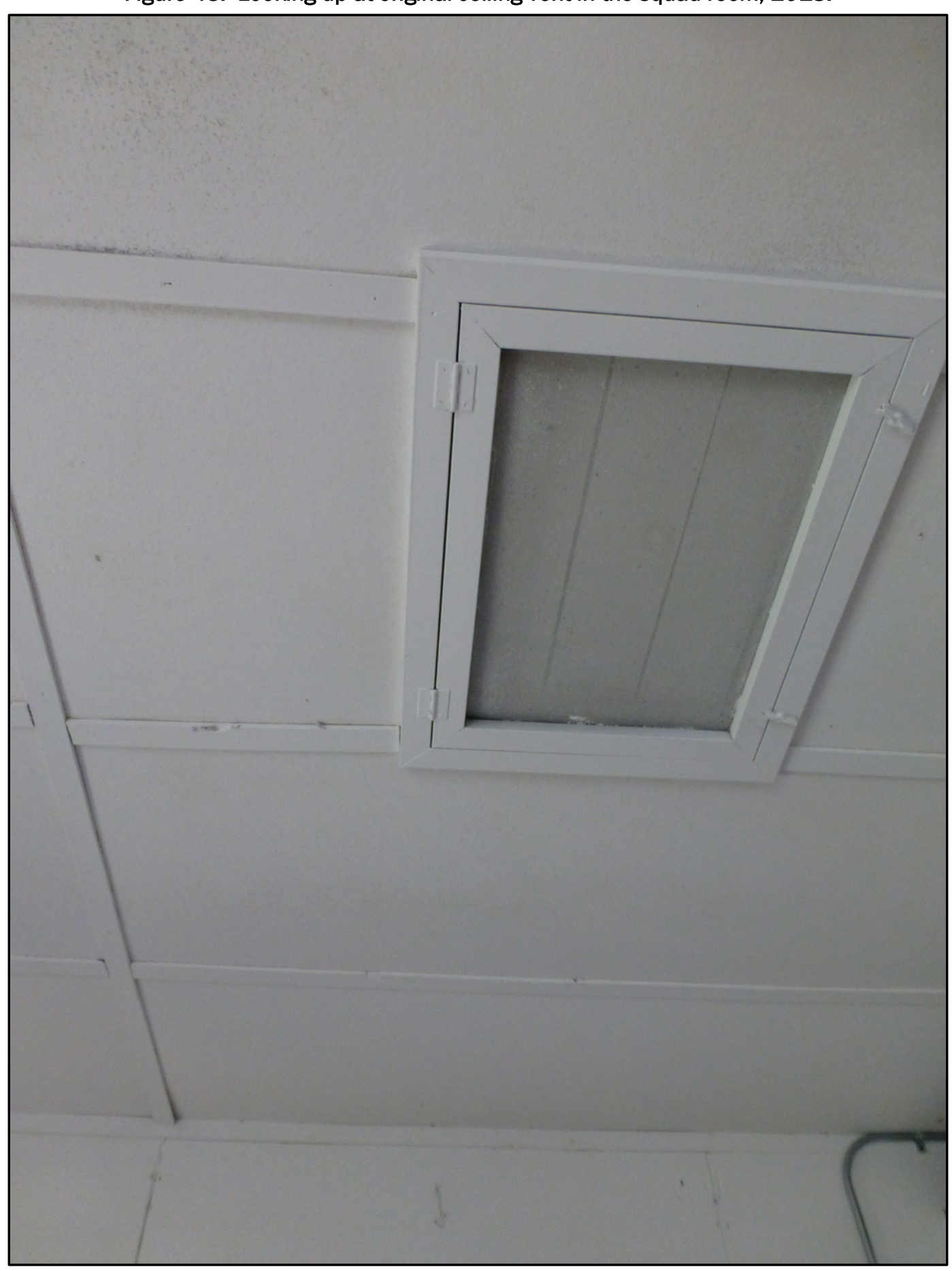




\section{Stage II - Building Zones}

Building zones establish the framework for planning for the operation, maintenance, and rehabilitation of an individual building by dividing the building into logical areas consistent with their use, original design, public access, and integrity. The concept of zoning, while establishing a logical framework, is also consistent with techniques of original architectural programming, design, and construction.

The zoning of the building seeks to identify the differences between more and less significant interior and exterior building areas and assigns a numerical rating, or level, to each zone. The zone ratings establish management and treatment requirements for each zone, i.e., highly significant public spaces may be in a "preservation zone" where maintenance is tightly controlled, and replacements are restricted. At the other end of the spectrum, larger, more private work areas may be subject to normal maintenance and open to a much broader range of architectural modification. The treatment guidelines for each level convey the general principles of preservation to be applied within the zone.

The six zones are:

- Level 1 - Preservation Zone (Red)

- Level 2 - Preservation Zone (Yellow)

- Level 3-Rehabilitation Zone (Green)

- Level 4 - Free Zone (White)

- Level 5- Hazardous Zone (Black Outline)

- Level 6 - Impact Zone (Red Stripes)

The Fort McCoy Fire Station, Bldg. 550, has three zones: Levels 2, 4, and 6 (see Figures 45 and 44).

\subsection{Level 2 - Preservation Zone}

Areas that exhibits distinguishing qualities or original materials and/or features, or representing examples of skilled craftsmanship. These areas are marked in yellow on the floor plan. Throughout the Bldg. 550 plan, there are features that are original from its construction in 1942. There are original wooden windows and doors, along with the trim, that are in poor condition but have the potential to be restored. There is major paint 
failure on most wood surfaces of Bldg. 550. The fire station has poured concrete floors and concrete block that makes up the foundation, but some areas are beginning to deteriorate. The floor begins to crack on corner areas which is most likely due to settling. The mortar failure within the concrete block could allow air, water, or pests to enter the building if not repaired. It is rare to find original light fixtures and bathroom fixtures in a building of this age. These fixtures should be cleaned appropriately and should be repaired if in a salvageable state. If non-repairable, the fixtures can be replaced with similar ones like those seen in the original 1942 plans. These features, if restored (or matched), will work to return Bldg. 550 to its true original character.

GUIDELINE: Preservation should be considered to allow the integrity of Bldg. 550's integrity to be preserved.

\subsection{Level 4 - Free Zone}

Level 4 areas are those not subject to the above three categories and whose removal or modification would not represent loss of character, code violation, or intrusion to an otherwise historically significant structure. Level 4 areas are colored white on the elevations and floor plan drawings.

Bldg. 550 once had a full brick chimney that extended out past the roof, but the portion that extended out was replaced with a metal chimney. The remainder of the chimney below the roof is original brick. Since the upper part is gone, there is no need to rebuild it. The portion that is left, which is in the interior, is in fair condition; therefore, it can be restored or replaced.

GUIDELINE: Preservation is always encouraged to keep a building similar to Bldg. 550 close to its original condition.

\subsection{Level 6 - Impact Zone}

These include areas that are improperly used and may result in code violations or areas insensitively adapted (see Figure 44), resulting in a general loss or concealment of character and/or loss or obscuring of significant historic fabric or features. Adequate existing fabric must be available to support or provide guidance for the rehabilitation of the zone and the restoration of the character of the original area. These areas are striped red on the elevations (Figure 45). On all facades of Bldg. 550, the original asbestos shingle siding with scalloped bottom edge siding, installed in 1942, 
was completely removed and the walls were covered in Tyvek paper in 2019. The removal of this siding caused a major character-defining quality of Bldg. 550 to be lost.

GUIDELINE: Since the original asbestos shingle siding with scalloped bottom edge siding on Bldg. 550 has been removed, it should be replaced with a siding similar to what was once used. This will restore a major part of the feeling and integrity of Bldg. 550 .

Figure 44. Floor plan of Bldg. 550 and key detailing of the zones of the building (Fort McCoy DPW modified by ERDC-CERL).

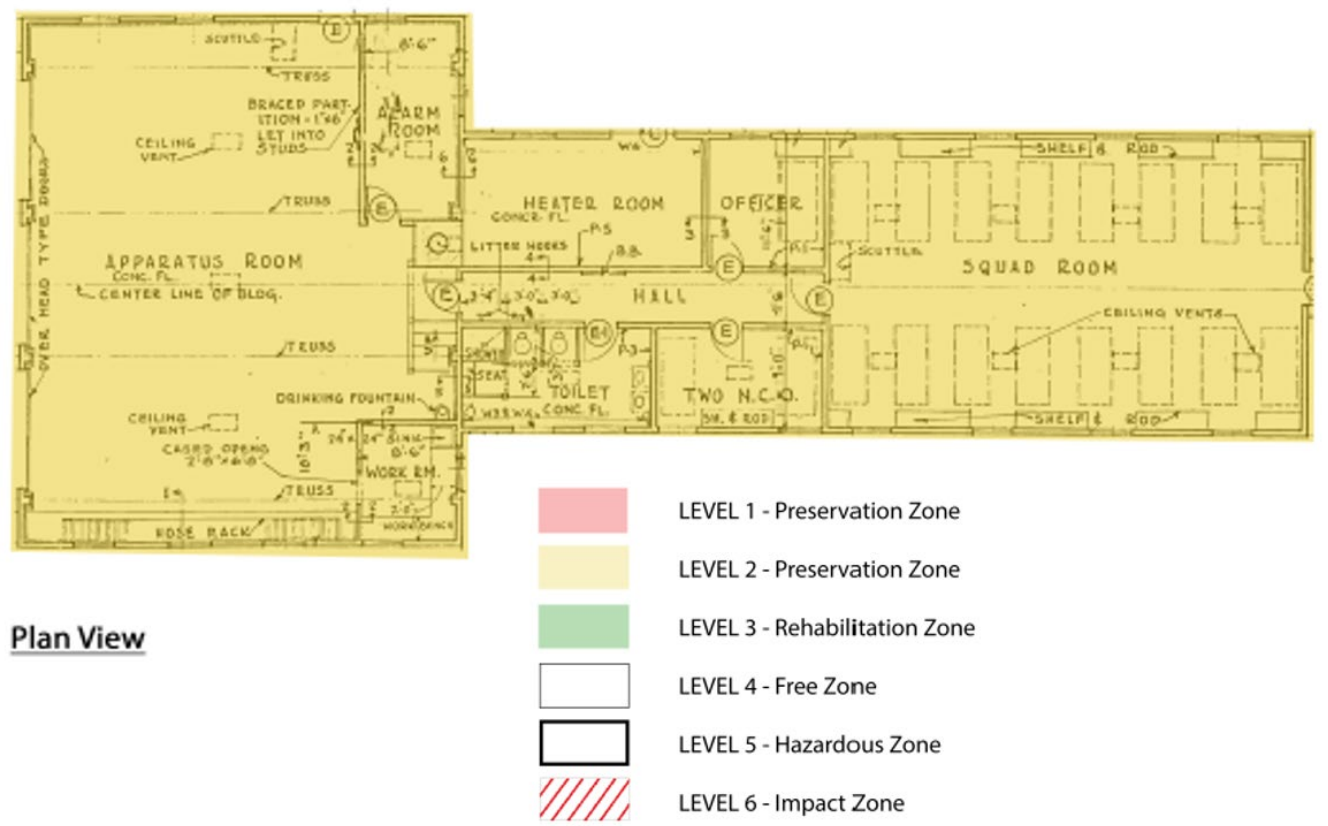

Source: Fort McCoy DPW (modified by ERDC-CERL). 
Figure 45. Elevations of Bldg. 550 and key detailing of the zones of the building for Levels 2, 4 , and 6.

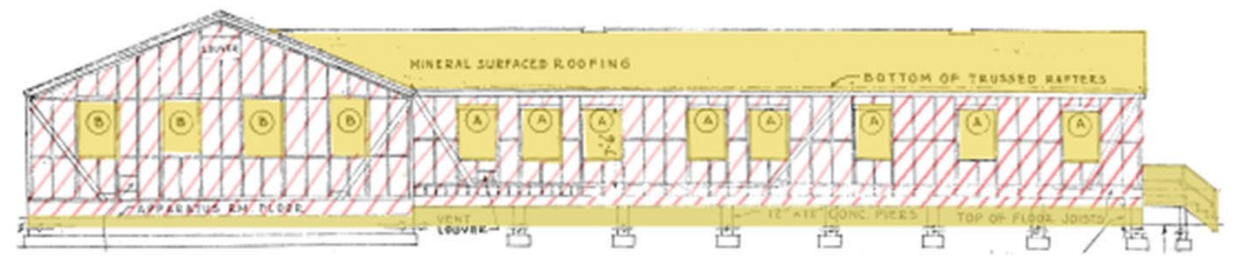

South Elevation
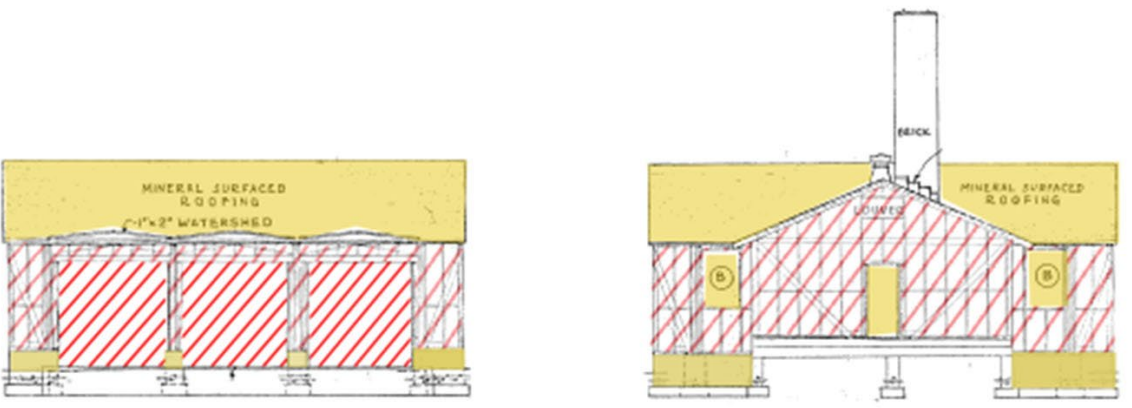

West Elevation

East Elevation

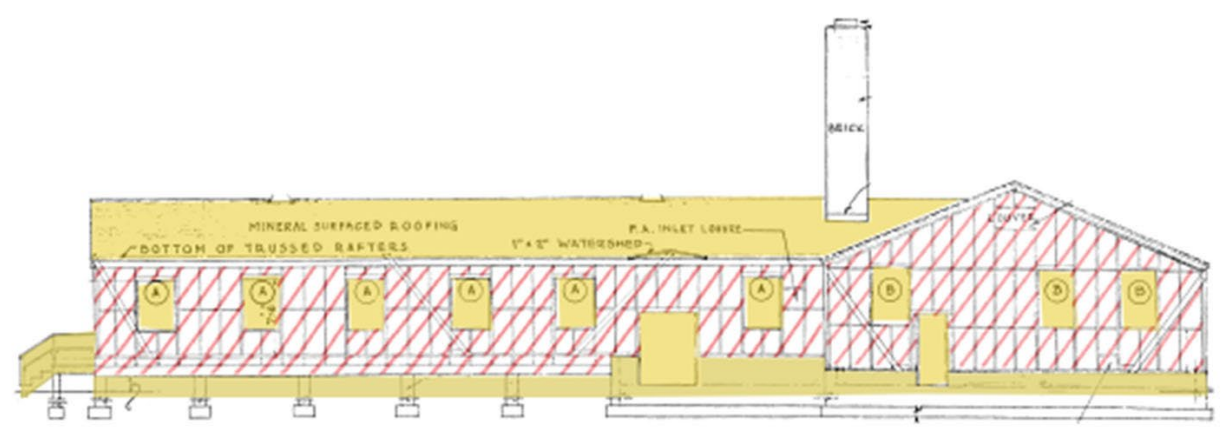

North Elevation

LEVEL 1 - Preservation Zone

LEVEL 2 - Preservation Zone

LEVEL 3 - Rehabilitation Zone

LEVEL 4 - Free Zone

LEVEL 5 - Hazardous Zone

LEVEL 6 - Impact Zone

Source: Fort McCoy DPW (modified by ERDC-CERL). 


\section{Stage III - Wood}

Discussed below are exterior wood surfaces that exist on or are a part of Bldg. 550. This building consists of exterior wood details that are both original and non-original. There is significant damage due to the wooden material's age and exposure to the outdoor elements.

\subsection{Exterior wood features}

At the base of the structure on the east end of Bldg. 550 (the living area/squad room) is a wooden foundation skirting (see Figure 46). This wooden skirting is character-defining. It hides the crawl space and the concrete pier foundation system under the squad room portion of Bldg. 550 . According to HABS documentation of WWII buildings at Fort McCoy, the wooden skirting would have been painted a bluish gray which the Bldg. 550 skirting is still painted with and the color needs to be matched.

The condition of the wooden foundation skirting is POOR. There is paint failure and some of the wood appears shifted and/or rotten.

Figure 46. Looking at wooden skirting on the northeast corner of Bldg. 550, 2018.

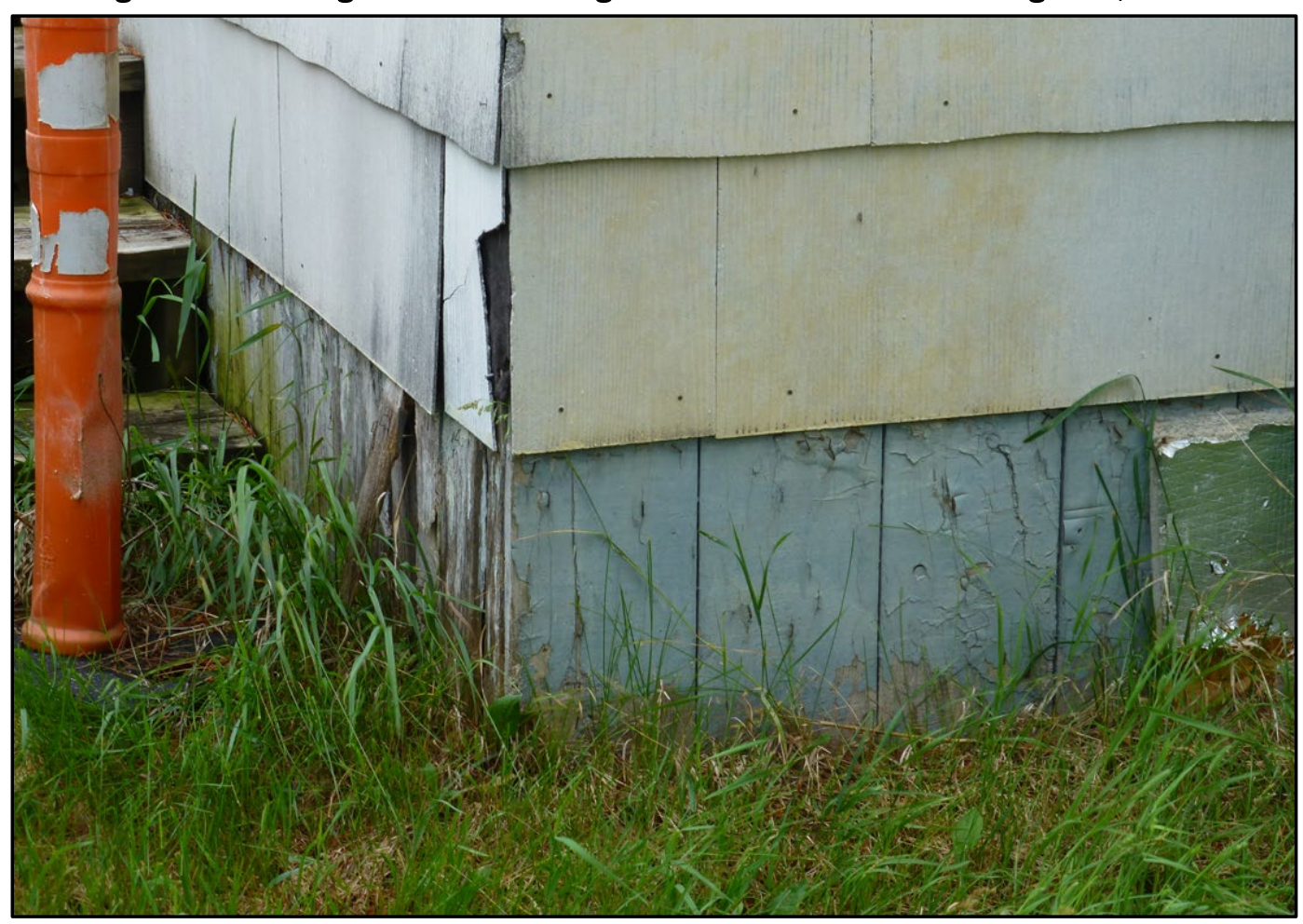


According to the original plans drafted in 1942, there was a set of wooden stairs and landing with wooden handrails to be built off of the rear door on the east elevation of Bldg. 550 (see Figure 47).

The condition of the wooden stairs, landing, and handrails is POOR (see Figure 48). These elements are not original to Bldg. 550. The wood appears rotten and possible damaged due to the outdoor elements, and the stairs land in the grass. The upper landing is also lacking a railing. These should be removed and replaced in-kind to the original drawing documentation.

Figure 47. Detail of original plans for wooden landing, steps, and handrails on the east side of Bldg. 550, 1942.

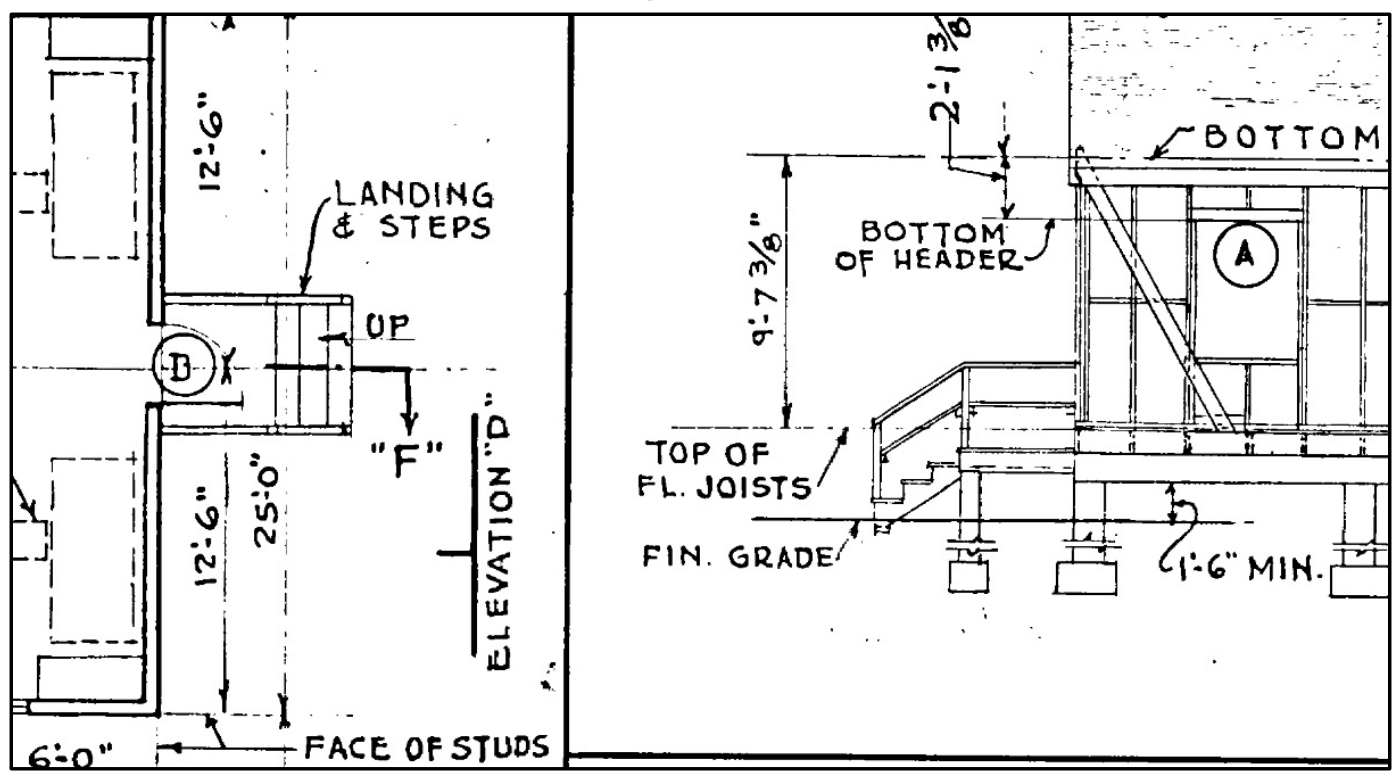

Source: Fort McCoy DPW. 
Figure 48. Looking at landing, stairs, and handrail on the east side of Bldg. 550, 2018.

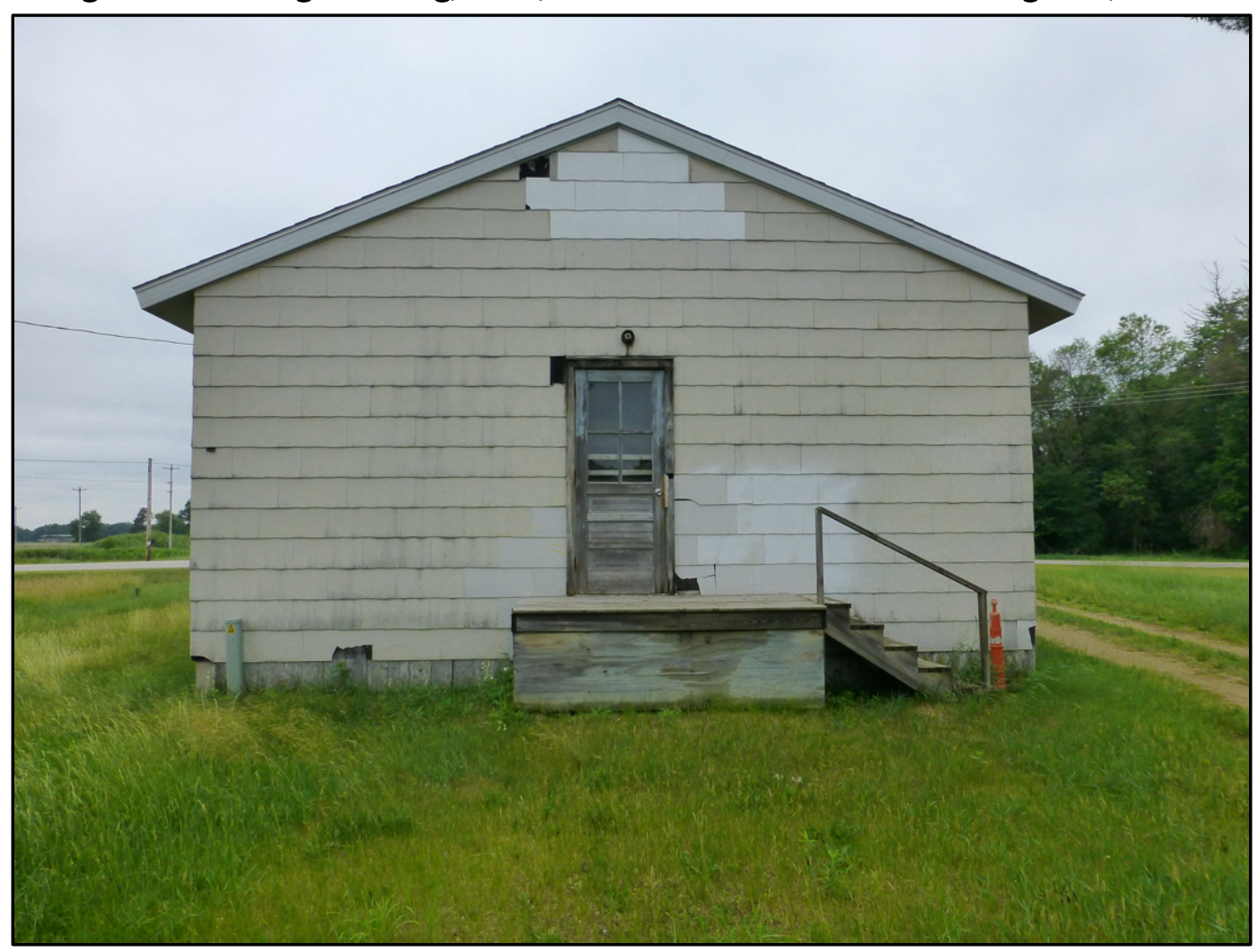

The wood window and door trim have the same $1 \frac{1 / 4}{}$ " $\times 3$ " flat board trim with a $1 \frac{1 / 4}{}$ " $\times 21 / 2$ " drip molding at the head. The condition of the wood trim is POOR. A 2018 image shown in Figure 49 demonstrates the significant damage to the exterior wood trim on Bldg. 550. There is obvious paint failure and the exposure to rain may have caused water damage and decaying. 
Figure 49. Looking at wooden window trim on the west side of Bldg. 550, 2018.

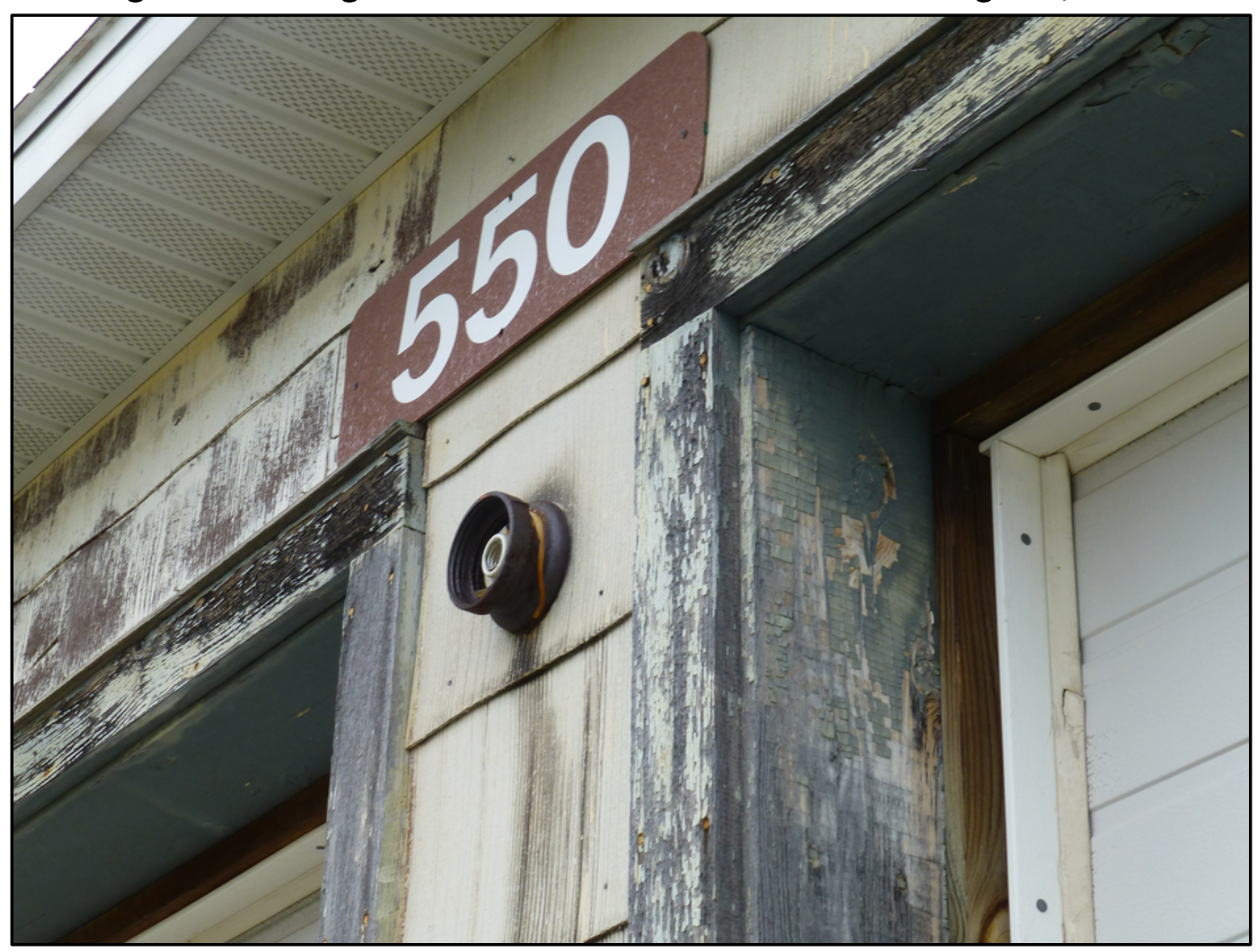

\subsection{Interior wood features}

Some of the interior wood features in Bldg. 550 include horizontally installed tongue and groove walls in the toilet room, wooden steps leading from the apparatus room to the squad area, wood shelves in the toilet room, wood trim around the ceiling vents, and battens on ceiling (see Figures 50 to 53 ).

The 2018 images shown in Figures 50 to 53 provides a visual representation of the condition of the interior wood features, which are in GOOD condition. Judging by the different colors, some portions of the interior wood features have been painted or replaced in past times. 
Figure 50. Original painted tongue and groove walls in the toilet room in Bldg. 550, 2018.

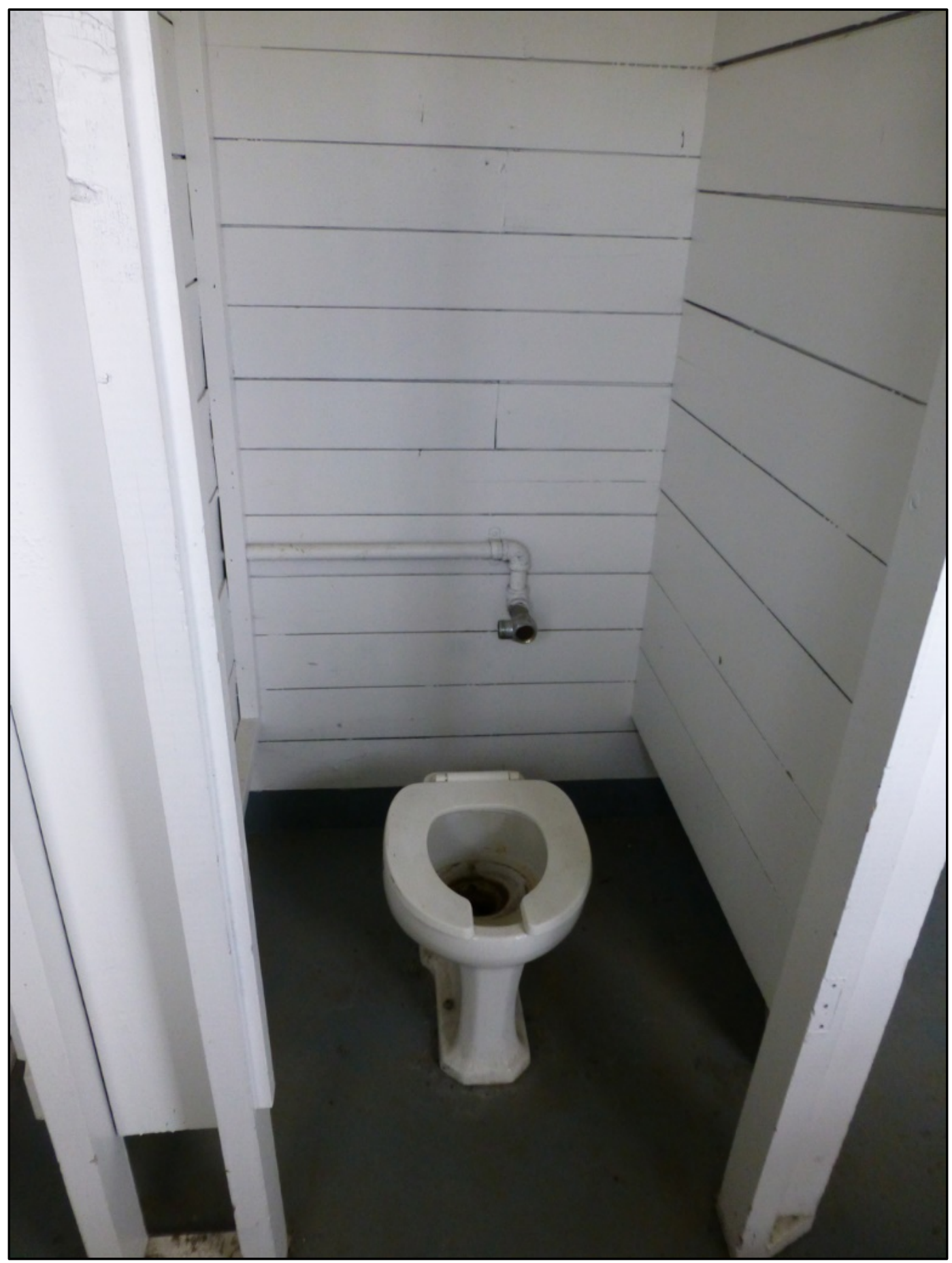


Figure 51. Wooden stair leading from the apparatus room to the squad area in Bldg. 550, 2018.

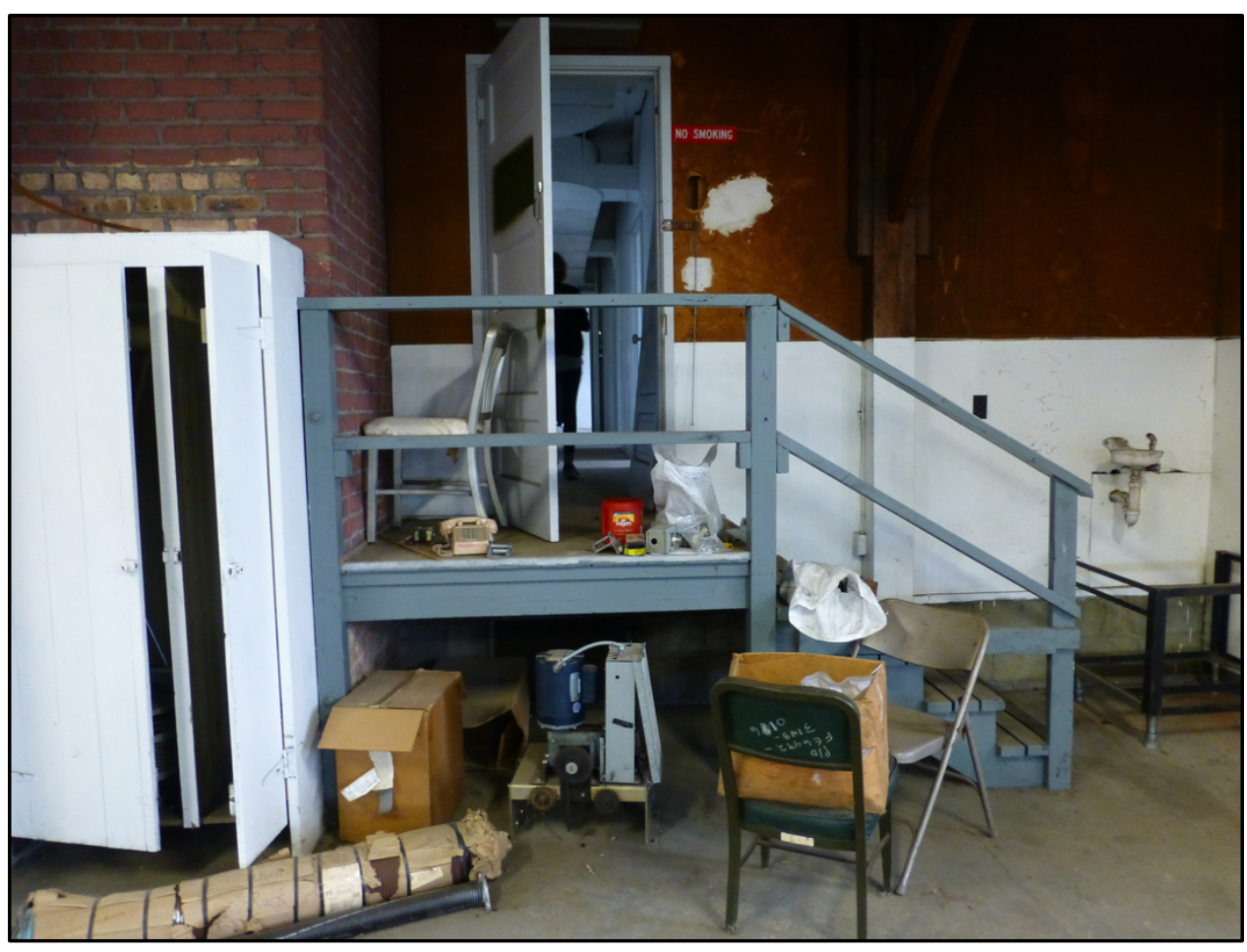

Figure 52. Original wood shelves in toilet room in Bldg. 550, 2018.

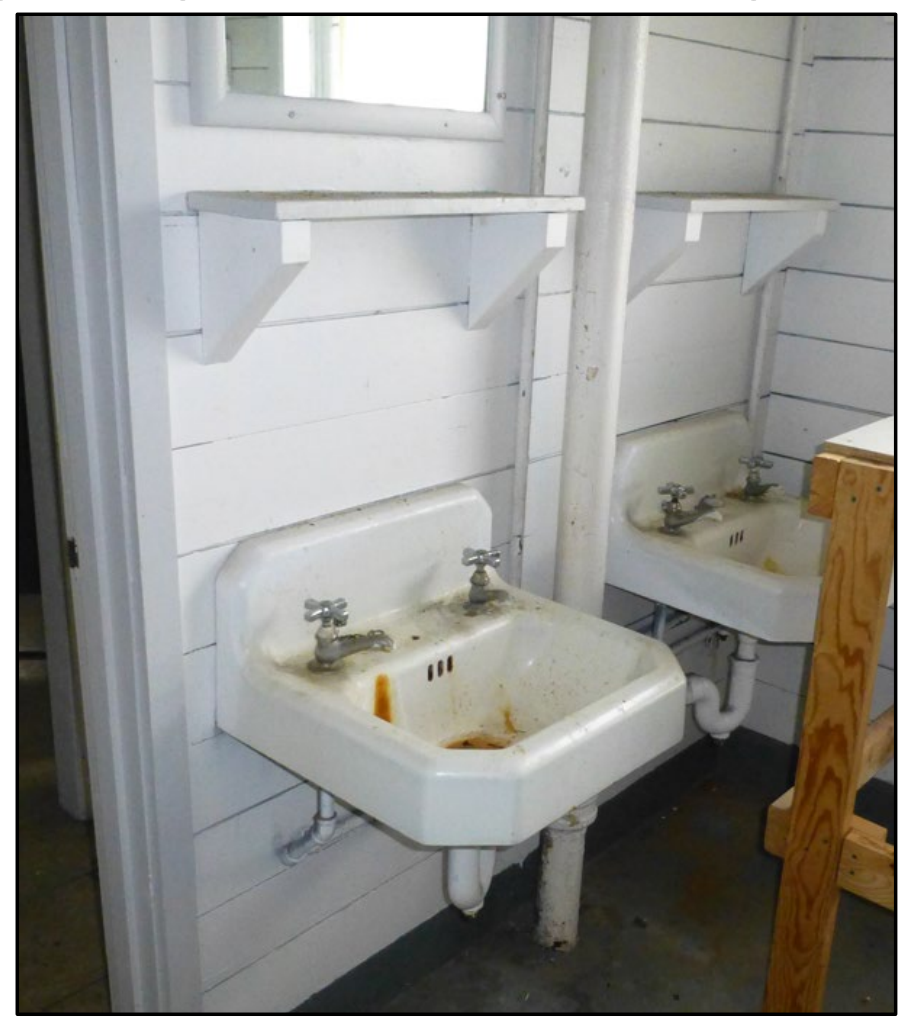


Figure 53. Looking up at the ceiling and wooden battens and wood trim on ceiling vent in the squad bay, 2018.

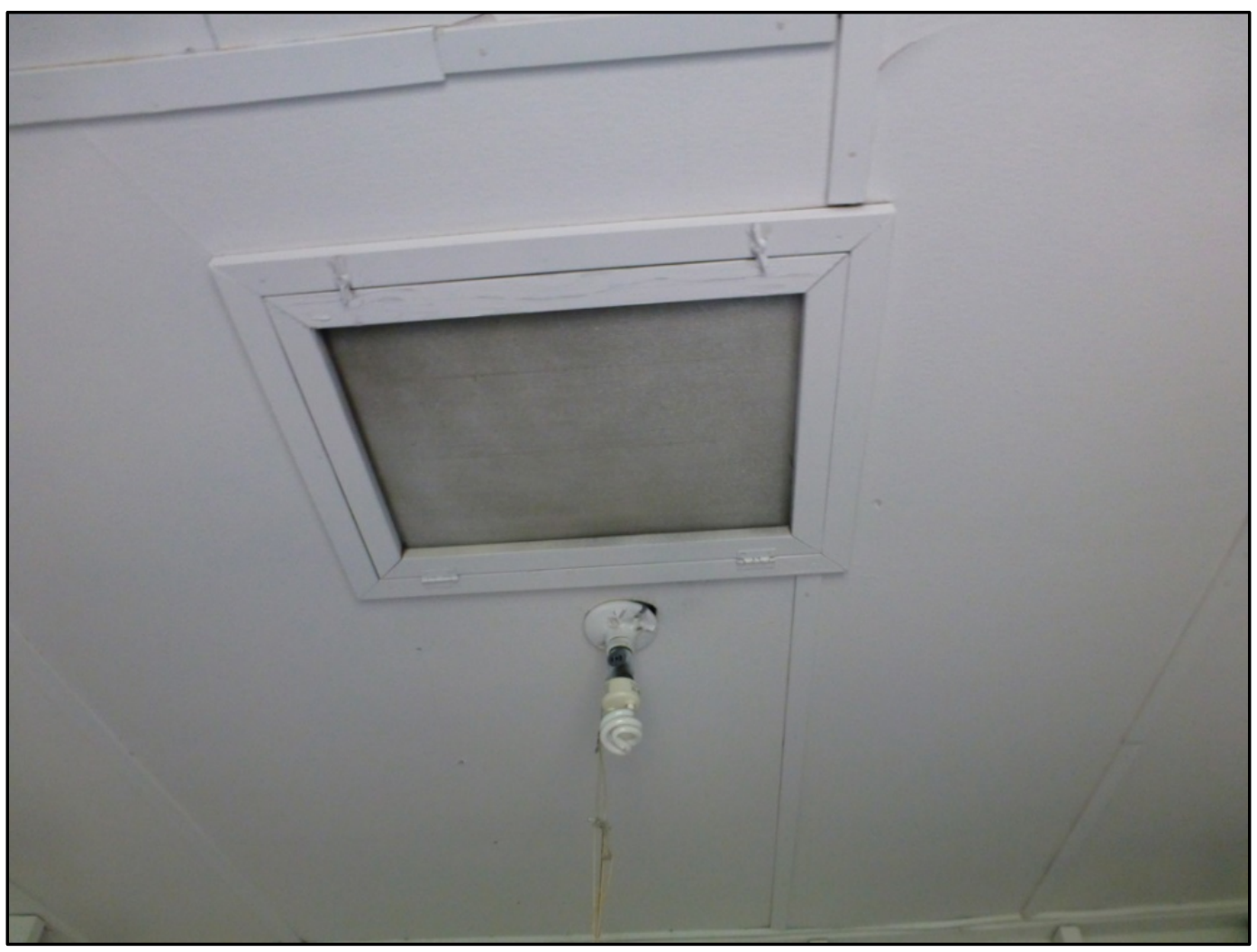

\subsection{Treatment measures for wood surfaces}

The following documents offer treatment measures for exterior and interior wood surfaces that are in poor condition. The sources include information from NPS.gov and GSA.gov. 


\subsubsection{Preservation Brief 10: Exterior paint problem on historic woodwork, 111982 (NPS.gov).}

Technical Preservation Services

PRESERVATION BRIEFS

10

\section{Exterior Paint Problems on Historic Woodwork}

Kay D. Weeks and David W. Look, AIA

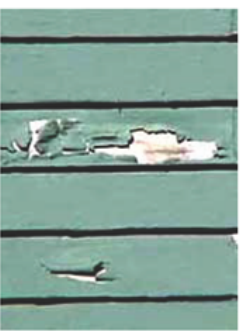

Peeling paint on historic wood siding. Photo: John Leeke,
2002.

\section{圆}

A cautionary approach to paint removal is included in the guidelines to the Secretary of the Interior Standards for Rehabilitation. Removing paints down to bare wood surfaces using harsh methods can permanently damage those surfaces; therefore such methods are not recommended. Also, total removal obliterates evidence of the historical paints and their sequence and architectural context.

This Brief expands on that advice for the architect, building manager, contractor, or homeowner by identifying and describing common types of paint surface conditions and failures, then recommending appropriate treatments for preparing exterior wood surfaces for repainting to assure the best adhesion and greatest durability of the new paint.

Although the Brief focuses on responsible methods of "paint removal," several paint surface conditions will be described which do not require any paint removal, and still others which can be successfully handled by limited paint removal. In all cases, the information is intended to address the concerns related to exterior wood. It will also be generally assumed that, because houses built before 1950 involve one or more layers of lead-based paint, the majority of conditions warranting paint removal will mean dealing with this toxic substance along with the dangers of the paint removal tools and chemical strippers themselves.

\section{Purposes of Exterior Paint}

Paint applied to exterior wood must withstand yearly extremes of both temperature and humidity. While never expected to be more than a temporary physical shield-requiring

reapplication every 5 to 8 years-its importance should not be minimized. Because one of the

11 Retrieved from: https://www.nps.gov/tps/how-to-preserve/briefs.htm. 
main causes of wood de terioration is moisture penetration, a primary purpose for painting wood is to exclude such moisture, thereby slowing deterioration not only of a building's exterior siding and decorative features but, ultimately, its underlying structural members. Another important purpose for painting wood is, of course, to define and accent architectural features and to improve appearance.

\section{Treating Paint Problems in Historic Buildings}

Exterior paint is constantly deteriorating through the processes of weathering, but in a program of regular maintenance-assuming all other building systems are functioning properly-surfaces can be cleaned, lightly scraped, and hand sanded in preparation for a new
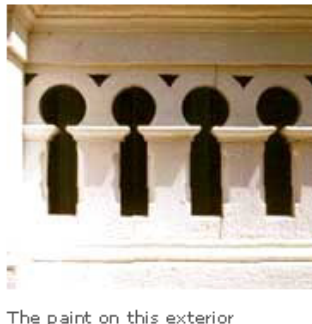

The paint on this exterior decorative feature is sound. Photo: NPS files. finish coat. Unfortunately, these are ideal conditions. More often, complex maintenance problems are inherited by owners of historic buildings, including areas of paint that have failed beyond the point of mere cleaning, scraping, and hand sanding (although much so-called "paint failure" is attributable to interior or exterior moisture problems or surface preparation and application mistakes with previous coats).

Although paint problems are by no means unique to historic buildings, treating multiple layers of hardened, brittle paint on complex, ornamental-and possibly fragile-exterior wood surfaces necessarily requires an extremely cautious approach. In the case of recent construction, this level of concern is not needed because the wood is generally less detailed and, in addition, retention of the sequence of paint layers as a partial record of the building's history is not an issue.

When historic buildings are involved, however, a special set of problems arises-varying in complexity depending upon their age, architectural style, historical importance, and physical soundness of the wood-which must be carefully evaluated so that decisions can be made that are sensitive to the longevity of the resource.

\section{Justification for Paint Removal}

At the outset of this Brief, it must be emphasized that removing paint from historic buildings -with the exception of cleaning, light scraping, and hand sanding as part of routine maintenance-should be avoided unless absolutely essential. Once conditions warranting removal have been identified the general approach should be to remove paint to the next sound layer using the gentlest means possible, then to repaint. Practically speaking as well, paint can adhere just as effectively to existing paint as to bare wood, providing the previous coats of paint are also adhering uniformly and tightly to the wood and the surface is properly prepared for repainting - cleaned of dirt and chalk and dulled by sanding.

But, if painted exterior wood surfaces display continuous patterns of deep cracks or if they are extensively blistering and peeling so that bare wood is visible, then the old paint should be completely removed before repainting. The only other justification for removing all previous layers of paint is if doors, shutters, or windows have literally been "painted shut," or if new wood is being pieced-in adjacent to old painted wood and a smooth transition is desired.

\section{Paint Removal Precautions}

Because paint removal is a difficult and painstaking process, a number of costly, regrettable experiences have occurredand continue to occur-for both the historic building and the building owner. Historic buildings have been set on fire with blow torches; wood irreversibly scarred by sandblasting or by harsh mechanical devices such as rotary sanders and rotary wire strippers; and layers of historic paint inadvertently and unnecessarily removed. In addition, property owners, using techniques that substitute speed for safety, have been injured by toxic lead vapors or dust from the paint they were trying to remove or by misuse of the paint removers themselves.

Owners of historic properties considering paint removal should also be aware of the amount of time and labor involved. While removing damaged layers of paint from a door or porch railing might be readily accomplished within a reasonable period of time by one or two people, removing paint from larger areas of a building can, without professional assistance, easily become unmanageable and produce less than satisfactory results. The amount of work involved in any paint removal project must therefore be analyzed on a case-by-case basis. Hiring qualified professionals will often be a cost-effective decision due to the expense of materials, the special equipment required, and the amount of time involved. Further, paint removal companies experienced in dealing with the inherent health and safety dangers of paint removal should have purchased such protective devices as are needed to mitigate any dangers and should also be aw are of State or local environmental and/or health regulations for hazardous waste disposal. 
All in all, paint removal is a messy, expensive, and potentially dangerous aspect of rehabilitating or restoring historic buildings and should not be undertaken without care ful thought concerning first, its necessity, and second, which of the available recommended methods is the safest and most appropriate for the job at hand.

\section{Repainting Historic Buildings for Cosmetic Reasons}

If existing exterior paint on wood siding, eaves, window sills, sash, and shutters, doors, and decorative features shows no evidence of paint deterioration such as chalking, blistering, peeling, or cracking, then there is no physical reason to repaint, much less remove paint! Nor is color fading, of itself, sufficient justification to repaint a historic building.

The decision to repaint may not be based altoge ther on paint failure. Where there is a new owner, or even where ownership has remained constant through the years, taste in colors often changes. Therefore, if repainting is primarily to alter a building's primary and accent colors, a technical factor of paint accumulation should be taken into consideration.

When paint builds up to a thickness of approximately $1 / 16$ " (approximately 16 to 30 layers), one or more extra coats of paint may be enough to trigger cracking and peeling in limited or even widespread areas of the building's surface. This results because excessively thick paint is less able to withstand the shrinkage or pull of an additional coat as it dries and is also less able to tolerate thermal stresses. Thick paint invariably fails at the weakest point of adhesion-the oldest layers next to the wood. Cracking and peeling follow. Therefore, if there are no signs of paint failure, it may be some what risky to add still another layer of unneeded paint simply for color's sake (extreme changes in color may also require more than one coat to provide proper hiding power and full color). When paint appears to be nearing the critical thickness, a change of accent colors (that is, just to limited portions of the trim) might be an acceptable compromise without chancing cracking and peeling of paint on wooden siding.

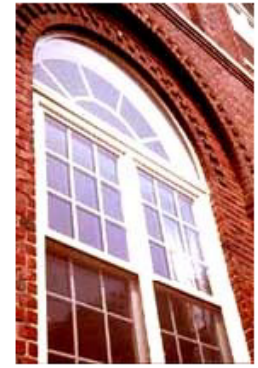

when the paint on the wood window s became too thick, it was removed and the window repainted. Photo: NPS files.

If the decision to repaint is nonetheless made, the "new" color or colors should, at a minimum, be appropriate to the style and setting of the building. On the other hand, where the intent is to restore or accurately reproduce the colors originally used or those from a significant period in the building's evolution, they should be based on the results of a paint analysis.

\section{Identification of Exterior Paint Surface Conditions/Recommended Treatments}

It is assumed that a preliminary check will already have been made to determine, first, that the painted exterior surfaces are indeed wood-and not stucco, metal, or other wood substitutes-and second, that the wood has not decayed so that repainting would be superfluous. For example, if any area of bare wood such as window sills has been exposed for a long period of time to standing water, wood rot is a strong possibility. Repair or replacement of deteriorated wood should take place before repainting. After the se two basic issues have been resolved, the surface condition identification process may commence.

The historic building will undoubtedly exhibit a variety of exterior paint surface conditions. For example, paint on the wooden siding and doors may be adhering firmly; paint on the eaves peeling; and paint on the porch balusters and window sills cracking and alligatoring. The accurate identification of each paint problem is therefore the first step in planning an appropriate overall solution.

Paint surface conditions can be grouped according to their relative severity: CLASS I conditions include minor blemishes or dirt collection and generally require no paint removal; CLASS II conditions include failure of the top layer or layers of paint and generally require

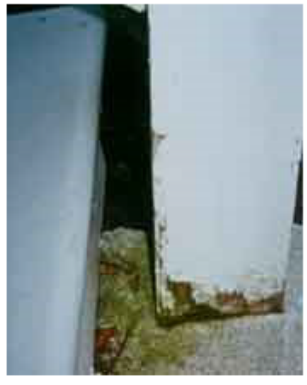

The problem evidenced here by mossy growth and deteriorated wood must be resolved and the wood allowed to dry out before the wood is repainted. Photo: NPS files.

limited paint removal; and CLASS III conditions include substantial or multiple-layer failure and generally require total paint removal. It is precisely because conditions will vary at different points on the building that a careful inspection is critical. Each item of painted exterior woodwork (i.e., siding, doors, windows, eaves, shutters, and decorative elements) should be examined early in the planning phase and surface conditions noted.

\section{CLASS I Exterior Surface Conditions Generally Requiring No Paint Removal Dirt, Soot, Pollution, Cobwebs, Insect Cocoons, etc.}

Cause of Condition

Environmental "grime" or organic matter that tends to cling to painted exterior surfaces and, in particular, protected 
surfaces such as eaves, do not constitute a paint problem unless painted over rather than removed prior to repainting. If not removed, the surface deposits can be a barrier to proper adhesion and cause peeling.

\section{Recommended Treatment}

Most surface matter can be loosened by a strong, direct stream of water from the nozzle of a garden hose. Stubborn dirt and soot will need to be scrubbed off using $1 / 2$ cup of household detergent in a gallon of water with a medium soft bristle brush. The cleaned surface should then be rinsed thoroughly, and permitted to dry before further inspection to determine if repainting is necessary. Quite often, cleaning provides a satisfactory enough result to postpone repainting.

\section{Mildew}

\section{Cause of Condition}

Mildew is caused by fungi feeding on nutrients contained in the paint film or on dirt adhering to any surface. Because moisture is the single most important factor in its growth, mildew tends to thrive in areas where dampness and lack of sunshine are problems such as window sills, under eaves, around gutters and downspouts, on the north side of buildings, or in shaded areas near shrubbery. It may sometimes be difficult to distinguish mildew from dirt, but there is a simple test to differentiate: if a drop of household bleach is placed on the suspected surface, mildew will immediately turn white whereas dirt will continue to look like dirt.

\section{Recommended Treatment}

Because mildew can only exist in shady, warm, moist areas, attention should be given to altering the environment that is conducive to fungal growth. The area in question may be shaded by trees which need to be pruned back to allow sunlight to strike the building; or may lack rain gutters or proper drainage at the base of the building. If the shady or moist conditions can be altered, the mildew is less likely to reappear. A recommend solution for removing mildew consists of one cup nonammoniated detergent, one quart household bleach, and one gallon water. When the surface is scrubbed with this solution using a medium soft brush, the mildew should disappear; however, for particularly stubborn spots, an additional quart of bleach may be added. After the area is mildew-free, it should then be rinsed with a direct stream of water from the nozzle of a garden hose, and permitted to dry thoroughly. When repainting, specially formulated "mildew-resistant" primer and finish coats should be used.

\section{Excessive Chalking}

\section{Cause of Condition}

Chalking-or powdering of the paint surface-is caused by the gradual disintegration of the resin in the paint film. (The amount of chalking is determined both by the formulation of the paint and the amount of ultraviolet light to which the paint is exposed.) In moderation, chalking is the ideal way for a paint to "age, ${ }_{r}$ because the chalk, when rinsed by rainwater carries discoloration and dirt away with it and thus provides an ideal surface for repainting. In excess, however $r_{r}$ it is not desirable because the chalk can wash down onto a surface of a different color beneath the painted area and cause streaking as well as rapid disintegration of the paint film itself. Also, if a paint contains too much pigment for the amount of binder (as the old white lead carbonate/oil paints often did), excessive chalking can result.

\section{Recommended Treatment}

The chalk should be cleaned off with a solution of $\mathrm{I} / 2$ cup household detergent to one gallon water, using a medium soft bristle brush. After scrubbing to remove the chalk, the surface should be rinsed with a direct stream of water from the nozzle of a garden hose, allowed to dry thoroughly, (but not long enough for the chalking process to recur) and repainted, using a non-chalking paint.

\section{Staining}

\section{Cause of Condition}

Staining of paint coatings usually results from excess moisture reacting with materials within the wood substrate. There are two common types of staining, neither of which requires paint removal. The most prevalent type of stain is due to the oxidation or rusting of iron nails or metal (iron, steel, or copper) anchorage devices. A second type of sta in is caused by a chemical reaction between moisture and natural extractives in certain woods (red cedar or redwood) which results in a surface deposit of colored matter. This is most apt to occur in new replacement wood within the first 10-15 years.

\section{Recommended Treatment}

In both cases, the source of the stain should first be located and the moisture problem corrected.

When stains are caused by rusting of the heads of nails used to attach shingles or siding to an exterior wall or by rusting or oxidizing iron, steel $_{r}$ or copper anchorage devices adjacent to a painted surface, the metal objects themselves should be hand sanded and coated with a rust-inhibitive primer followed by two finish coats. (Exposed nail heads should ideally be countersunk, spot primed, and the holes filled with a high quality wood filler except where exposure of the nail head was part of the original construction system or the wood is too fragile to withstand the countersinking procedure.) 
Discoloration due to color extractives in replacement wood can usually be cleaned with a solution of equal parts denatured alcohol and water. After the affected area has been rinsed and permitted to dry, a "stainblocking primer" especially developed for preventing this type of stain should be applied (two primer coats are recommended for severe cases of bleeding prior to the finish coat). Each primer coat should be allowed to dry at least 48 hours.

\section{CLASS II Exterior Surface Conditions Generally Requiring Limited Paint Removal \\ Crazing}

Cause of Condition

Crazing-fine, jagged interconnected breaks in the top layer of paint-results when paint that is several layers thick becomes excessively hard and brittle with age and is consequently no longer able to expand and contract with the wood in response to changes in temperature and humidity. As the wood swells, the bond between paint layers is broken and hairline cracks appear. Al though somewhat more difficult to detect as opposed to other more obvious paint problems, it is well worth the time to scrutinize all surfaces for crazing. If not corrected, exterior moisture will enter the crazed surface, resulting in further swelling of the wood and, eventually, deep cracking and alligatoring, a Class III condition which requires total paint removal.

\section{Recommended Treatment}

Crazing can be treated by hand or mechanically sanding the surface, then repainting. Although the hairline cracks may tend to show through the new paint, the surface will be protected against

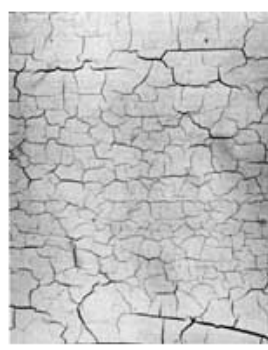

Crazing-or surface cracking -is an exterior surface condition which can be succes fully treated by anding and painting. sanding and painting. Photo: Courtesy, National Decor ating Products Assocation. exterior moisture penetration.

\section{Intercoat Peeling}

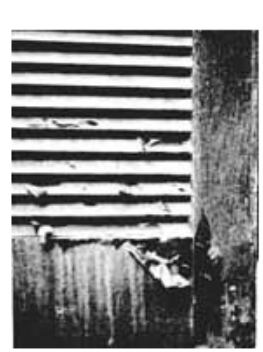

Here, a latex top coat $w$ as applied directly over old oil paint, resulting in intercoat peeling. The latex was unable to adhere. If latex is used over oil, an oil-base primer should be applied first. Photo: Mary L, Oehrlein, AIA.

\section{Cause of Condition}

Intercoat peeling can be the result of improper surface preparation prior to the last repainting. This most often occurs in protected areas such as eaves and covered porches because these surfaces do not receive a regular rinsing from rainfall, and salts from airborne pollutants thus accumulate on the surface. If not cleaned off, the new paint coat will not adhere properly and that layer will peel.

Another common cause of intercoat peeling is incompatibility between paint types. For example, if oil paint is applied over latex paint, peeling of the top coat can sometimes result since, upon aging, the oil paint becomes harder and less elastic than the latex paint. If latex paint is applied over old, chalking oil paint, peeling can also occur because the latex paint is unable to penetrate the chalky surface and adhere.

\section{Recommended Treatment}

First, where salts or impurities have caused the peeling, the affected area should be washed down thoroughly after scraping, then wiped dry. Finally, the surface should be hand or mechanically sanded, then repainted.

Where peeling was the result of using incompatible paints, the peeling top coat should be scraped and hand or mechanically sanded. Application of a high quality oil type exterior primer will provide a surface over which either an oil or a latex topcoat can be successfully used.

\section{Solvent Blistering}

\section{Cause of Condition}

Solvent blistering, the result of a less common application error, is not caused by moisture, but by the action of ambient heat on paint solvent or thinners in the paint film. If solventrich paint is applied in direct sunlight, the top surface can dry too quickly and, as a result, solvents become trapped beneath the dried paint film. When the solvent vaporizes, it forces its way through the paint film, resulting in surface blisters. This problem occurs more often with dark colored paints because darker colors absorb more heat than lighter ones. To distinguish between solvent blistering and blistering caused by moisture, a blister should be cut open. If another layer of paint is visible, then solvent blistering is likely the problem whereas if bare wood is revealed, moisture is probably to blame. Solvent blisters are generally small.

\section{Recommended Treatment}

Solvent-blistered areas can be scraped, hand or mechanically sanded to the next sound layer, then repainted. In order to prevent blistering of painted surfaces, paint should not be applied in direct sunlight. 


\section{Wrinkling}

Cause of Condition

Another error in application that can easily be avoided is wrinkling. This occurs when the top layer of paint dries before the layer underneath. The top layer of paint actually moves as the paint underneath (a primer, for example) is drying. Specific causes of wrinkling include: (1) applying paint too thick; (2) applying a second coat before the first one dries; (3) inadequate brushing out; and (4) painting in temperatures higher than recommended by the manufacturer.

Recommended Treatment

The wrinkled layer can be removed by scraping followed by hand or mechanical sanding to provide as even a surface as possible, then repainted following manufacturer's application instructions.

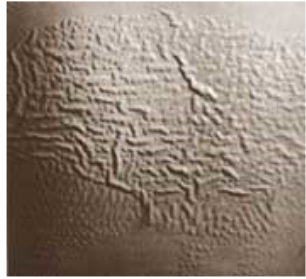

Wrinkled layers can generally be removed by scraping and sanding as opposed to total paint removal. Photo: Courtesy, National Decorating Products Association.

\section{CLASS III Exterior Surface Conditions Generally Requiring Total Paint Removal}

If surface conditions are such that the majority of paint will have to be removed prior to repainting, it is suggested that a small sample of intact paint be left in an inconspicuous area either by covering the area with a metal plate, or by marking the area and identifying it in some way. (When repainting does take place, the sample should not be painted over). This will enable future investigators to have a record of the building's paint history.

\section{Peeling}

\section{Cause of Condition}

Peeling to bare wood is most often caused by excess interior or exterior moisture that collects behind the paint film, thus impairing adhesion. Generally beginning as blisters, cracking and peeling occur as moisture causes the wood to swell, breaking the adhesion of the bottom layer.

\section{Recommended Treatment}

There is no sense in repainting before dealing with the moisture problems because new paint will simply fail. Therefore, the first step in treating peeling is to locate and remove the source or sources of the moisture, not only because moisture will jeopardize the protective coating of paint but because, if left unattended, it can ultimately cause permanent damage to the wood. Excess interior moisture should be removed from the building through installation of exhaust fans and vents. Exterior moisture should be eliminated by correcting the following conditions

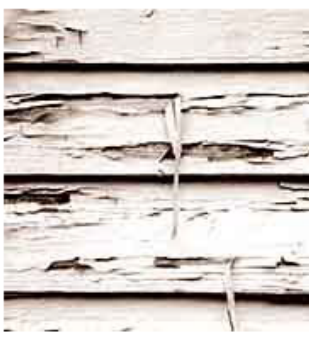

Extensively deteriorated paint needs to be removed to bare wood, then primed and repainted. Photo: NPS files. prior to repainting: faulty flashing; leaking gutters; defective roof shingles; cracks and holes in siding and trim; deteriorated caulking in joints and seams; and shrubbery growing too close to painted wood. After the moisture problems have been solved, the wood must be permitted to dry out thoroughly. The damaged paint can then be scraped off with a putty knife, hand or mechanically sanded, primed, and repainted.

\section{Cracking/Alligatoring}

Cause of Condition

Cracking and alligatoring are advanced stages of crazing. Once the bond between layers has been broken due to intercoat paint failure, exterior moisture is able to penetrate the surface cracks, causing the wood to swell and deeper cracking to take place.

This process continues until cracking, which forms parallel to grain, extends to bare wood. Ultimately, the cracking becomes an overall pattern of horizontal and vertical breaks in the paint layers that looks like reptile skin; hence, "alligatoring." In advanced stages of cracking and alligatoring, the surfaces will also flake badly.

\section{Recommended Treatment}

If cracking and alligatoring are present only in the top layers they can probably be scraped, hand or mechanically sanded to the next sound layer, then repainted. However, if cracking and/or alligatoring have progressed to bare wood and the paint has begun to flake, it will need to be totally removed. Methods include scraping or paint removal with the electric heat plate, electric heat gun, or chemical strippers, depending on the particular area involved. Bare wood should be primed within 48 hours then repainted.

\section{Selecting the Appropriate/Safest Method to Remove Paint}

After having presented the "hierarchy" of exterior paint surface conditions-from a mild condition such as mildewing which simply requires cleaning prior to repainting to serious conditions such as peeling and alligatoring which require total paint removal-one important thought bears repeating: if a paint problem has been identified that warrants either limited or total 
paint removal, the gentlest method possible for the particular wooden element of the historic building should be selected from the many available methods.

The treatments recommended-based upon field testing as well as onsite monitoring of Department of Interior grant-in-aid and certification of rehabilitation projects-are therefore those which take three overriding issues into consideration (1) the continued protection and preservation of the historic exterior woodwork; (2) the retention of the sequence of historic paint layers; and (3) the health and safety of those individuals performing the paint removal. By applying these criteria, it will be seen that no paint removal method is without its drawbacks and all recommendations are qualified in varying degrees.

\section{Methods for Removing Paint}

After a particular exterior paint surface condition has been identified, the next step in planning for repainting-if paint removal is required-is selecting an appropriate method for such removal.

The method or methods selected should be suitable for the specific paint problem as well as the particular wooden element of the building. Methods for paint removal can be divided into three categories (frequently, however, a combination of the three methods is used). Each method is defined below, then discussed further and specific recommendations made:

- Abrasive-"Abrading" the painted surface by manual and/or mechanical means such as scraping and sanding. Generally used for surface preparation and limited paint removal.

- Thermal-Softening and raising the paint layers by applying heat followed by scraping and sanding. Generally used for total paint removal.

- Chemical-Softening of the paint layers with chemical strippers followed by scraping and sanding. Generally used for total paint removal.

\section{Abrasive Methods (Manual)}

If conditions have been identified that require limited paint removal such as crazing, intercoat peeling, solvent blistering, and wrinkling, scraping and hand sanding should be the first methods employed before using mechanical means. Even in the case of more serious conditions such as peeling-where the damaged paint is weak and already sufficiently loosened from the wood surface - scraping and hand sanding may be all that is needed prior to repainting.

\section{Recommended Abrasive Methods (Manual)}

Putty Knife/Paint Scraper: Scraping is usually accomplished with either a putty knife or a paint scraper, or both. Putty knives range in width from one to six inches and have a beveled edge. A putty knife is used in a pushing motion going under the paint and working from an area of loose paint toward the edge where the paint is still firmly adhered and, in effect, "beveling" the remaining layers so that as smooth a transition as possible is made between damaged and undamaged areas.

Paint scrapers are commonly available in $1-5 / 16,2-1 / 2$, and 3-1/2 inch widths and have replaceable blades. In addition, profiled scrapers can be made specifically for use on moldings. As opposed to the putty knife, the paint scraper is used in a pulling motion and works by raking the damaged areas of paint away.

The obvious goal in using the putty knife or the paint scraper is to selectively remove the affected layer or layers of paint; however, both of these tools, particularly the paint scraper with its hooked edge, must be used with care to properly prepare the surface and to avoid gouging the wood.

Sandpaper/Sanding Block/Sanding sponge: After manually removing the damaged layer or layers by scraping, the uneven surface (due to the almost inevitable removal of varying numbers of paint layers in a given area) will need to be smoothed or "feathered out" prior to repainting. As stated before, hand sanding, as opposed to harsher mechanical sanding, is recommended if the area is relatively limited. A coarse grit, open-coat flint sandpaper-the least expensive kind -is useful for this purpose because, as the sandpaper clogs with paint it must be discarded and this process repeated until all layers adhere uniformly.

Blocks made of wood or hard rubber and covered with sandpaper are useful for handsanding flat surfaces. Sanding sponges -rectangular sponges with an abrasive aggregate on their surfaces-are also available for detail work that requires reaching into grooves because the sponge easily conforms to curves and irregular surfaces. All sanding should be done with the grain.

\section{Summary of Abrasive Methods (Manual)}

- Recommended: Putty knife, paint scraper, sandpaper, sanding block, sanding sponge.

- Applicable areas of building: All areas. For use on: Class I, Class II and Class III conditions.

- Health/Safety factors: Take precautions against lead dust, eye damage; dispose of lead paint residue properly. 


\section{Abrasive Methods (Mechanical)}

If hand sanding for purposes of surface preparation has not been productive or if the affected area is too large to consider hand sanding by itself, mechanical abrasive methods, i.e., power-operated tools may need to be employed; however $r_{r}$ it should be noted that the majority of tools available for paint removal can cause damage to fragile wood and must be used with great care.

\section{Recommended Abrasive Methods (Mechanical)}

Orbital sander: Designed as a finishing or smoothing tool-not for the removal of multiple layers of paint-the orbital sander is thus recommended when limited paint removal is required prior to repainting. Because it sands in a small diameter circular motion (some models can also be switched to a back-and-forth vibrating action), this tool is particularly effective for "feathering" areas where paint has first been scraped. The abrasive surface varies from about $3 \times 7$ inches to $4 \times 9$ inches and sandpaper is attached either by clamps or sliding clips. A medium grit, open-coat aluminum oxide sandpaper should be used; fine sandpaper clogs up so quickly that it is ineffective for smoothing paint.

Belt sander: A second type of power tool-the belt sander-can also be used for removing limited layers of paint but, in this case, the abrasive surface is a continuous belt of sandpaper that travels at high speeds and consequently offers much less control than the orbital sander. Because of the potential for more damage to the paint or the wood, use of the belt sander (also with a medium grit sandpaper) should be limited to flat surfaces and only skilled operators should be permitted to operate it within a historic preservation project.

\section{Not Recommended}

Rotary Drill Attach ments: Rotary drill attachments such as the rotary sanding disc and the rotary wire stripper should be avoided. The disc sander-usually a disc of sandpaper about 5 inches in diameter secured to a rubber based attachment which is in turn connected to an electric drill or other motorized housing-can easily leave visible circular depressions in the wood which are difficult to hide, even with repainting. The rotary wire stripper-clusters of metals wires similarly attached to an electric drill-type unit-can actually shred a wooden surface and is thus to be used exclusively for removing corrosion and paint from metals.

Waterblasting: Waterblasting above 600 p.s.i. to remove paint is not recommended because it can force water into the woodwork rather than cleaning loose paint and grime from the surface; at worst, high pressure waterblasting causes the water to penetrate exterior sheathing and damages interior finishes. A detergent solution, a medium soft bristle brush, and a garden hose for purposes of rinsing, is the gentlest method involving water and is recommended when cleaning exterior surfaces prior to repainting.

Sandblasting: Finally-and undoubtedly most vehemently "not recommended"-sandblasting painted exterior woodwork will indeed remove paint, but at the same time can scar wooden elements beyond recognition. As with rotary wire strippers, sandblasting erodes the soft porous fibers (spring wood) faster than the hard, dense fibers (summer wood), leaving a pitted surface with ridges and valleys. Sandblasting will also erode projecting areas of carvings and moldings before it removes paint from concave areas. Hence, this abrasive method is potentially the most damaging of all possibilities, even if a contractor promises that blast pressure can be controlled so that the paint is removed without harming the historic exterior woodwork. (For Additional Information, See Preservation Briefs 6 , "Dangers of Abrasive Cleaning to Historic Buildings".)

\section{Summary of Abrasive Methods (Mechanical)}

- Recommended: Orbital sander, belt sander (skilled operator only).

- Applicable areas of building: Flat surfaces, i.e., siding, eaves, doors, window sills.

- For use on: Class II and Class III conditions.

- Health/Safety factors: Take precautions against lead dust and eye damage; dispose of lead paint residue properly.

- Not Recommended: Rotary drill attachments, high pressure waterblasting, sandblasting.

\section{Thermal Methods}

Where exterior surface conditions have been identified that warrant total paint removal such as peeling, cracking, or alligatoring, two thermal devices-the electric heat plate and the electric heat gun-have proven to be quite successful for use on different wooden elements of the historic building. One thermal method-the blow torch-is not recommended because it can scorch the wood or even burn the building down!

\section{Recommended Thermal Methods}

Electric heat plate: The electric heat plate operates between 500 and 800 degrees Fahrenheit (not hot enough to vaporize lead paint), using about 15 amps of power. The plate is held close to the painted exterior surface until the layers of paint begin to soften 


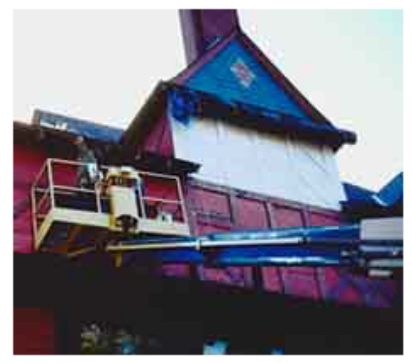

A heat plate $w$ as used on the cornice to remove paint. Photo: NPS files.

and blister, then moved to an adjacent location on the wood while the softened paint is scraped off with a putty knife (it should be noted that the heat plate is most successful when the paint is very thick!). With practice, the operator can successfully move the heat plate evenly across a flat surface such as wooden siding or a window sill or door in a continuous motion, thus lessening the risk of scorching the wood in an attempt to reheat the edge of the paint sufficiently for effective removal. Since the electric heat plate's coil is "red hot," extreme caution should be taken to avoid igniting clothing or burning the skin. If an extension cord is used, it should be a heavy-duty cord (with 3prong grounded plugs). A heat plate could overload a circuit or, even worse, cause an electrical fire; therefore, it is recommended that this implement be used with a single circuit and that a fire extinguisher always be kept close at hand.

Electric heat gun: The electric heat gun (electric hot-air gun) looks like a hand-held hairdryer with a heavy-duty metal case. It has an electrical resistance coil that typically he ats between 500 and 750 degrees Fahrenheit and, again, uses about 15 amps of power which requires a heavy-duty extension cord. There are some heat guns that operate at higher temperatures but they should not be purchased for removing old paint because of the danger of lead paint vapors.

The temperature is controlled by a vent on the side of the heat gun. When the vent is closed, the heat increases. A fan forces a stream of hot air against the painted woodw ork, causing a blister to form. At that point, the softened paint can be peeled back with a putty knife. It can be used to best advantage when a paneled door was originally varnished, then painted a number of times. In this case, the paint will come off quite easily, often leaving an almost pristine varnished surface behind. Like the heat plate, the heat gun works best on a heavy paint buildup. (It is, however, not very successful on only one or two layers of paint or on surfaces that have only been vamished. The varnish simply becomes sticky and the wood scorches.)

Although the heat gun is heavier and more tiring to use than the heat plate, it is particularly effective for removing paint from detail work because the nozzle can be directed at curved and intricate surfaces. Its use is thus more limited than the heat plate, and most successfully used in conjunction with the heat plate. For example, it takes about two to three hours to strip a paneled door with a heat gun, but if used in combination with a heat plate for the large, flat area, the time can usually be cut in half. Although a heat gun

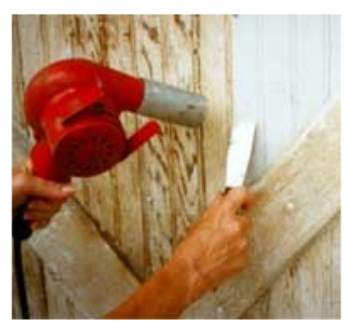

The nozzle on the electric heat gun permits hot air to be aimed into cavities on solid decorative surfaces, such as this carriage house door. After the paint has been sufficiently softened, it can be carefully removed with a scraper. Photo: NPS remo
files. seldom scorches wood, it can cause fires (like the blow torch) if aimed at the dusty cavity between the exterior sheathing and siding and interior lath and plaster. A fire may smolder for hours before flames break through to the surface. Therefore, this thermal device is best suited for use on solid decorative elements, such as molding, balusters, fre twork, or "gingerbread."

\section{Not Recommended}

Blow Torch: Blow torches, such as hand-held propane or butane torches, were widely used in the past for paint removal because other thermal devices were not available. With this technique, the flame is directed toward the paint until it begins to bubble and loosen from the surface. Then the paint is scraped off with a putty knife. Although this is a relatively fast process, at temperatures between 3200 and 3800 degrees Fahrenheit the open flame is not only capable of buming a careless operator and causing severe damage to eyes or skin, it can easily scorch or ignite the wood. The other fire hazard is more insidious. Most frame buildings have an air space between the exterior sheathing and siding and interior lath and plaster. This cavity usually has an accumulation of dust which is also easily ignited by the open flame of a blow torch. Finally, leadbase paints will vaporize at high temperatures, releasing toxic fumes that can be unknowingly inhaled. Therefore, because both the heat plate and the heat gun are generally safer to use - that is, the risks are much more controllable-the blow' torch should de finitely be avoided!

\section{Summary of Thermal Methods}

- Recommended: Electric heat plate, electric heat gun.

- Applicable areas of building: Electric heat plate-flat surfaces such as siding, eaves, sash, sills, doors. Electric heat gun-solid decorative molding, balusters, fretwork, or "gingerbread."

- For use on: Class III conditions.

- Health/Safety factors: Take precautions against eye damage and fire. Dispose of lead paint residue properly.

- Not Recommended: Blow torch. 


\section{Chemical Methods}

With the availability of effective thermal methods for total paint removal, the need for chemical methods-in the context of preparing historic exterior woodwork for repainting-becomes quite limited. Solvent-base or caustic strippers may, however play a supplemental role in a number of situations, including:

- Removing paint residue from intricate decorative features, or in cracks or hard to reach areas if a heat gun has not been completely effective;

- Removing paint on window muntins because heat devices can easily break the glass;

- Removing varnish on exterior doors after all layers of paint have been removed by a heat plate/heat gun if the original varnish finish is being restored;

- Removing paint from detachable wooden elements such as exterior shutters, balusters, columns, and doors by dip stripping when other methods are too laborious.

\section{Recommended Chemical Methods (Use With Extreme Caution)}

Because all chemical paint removers can involve potential health and safety hazards, no wholehearted recommendations can be made from that standpoint. Commonly known as "paint removers" or "strippers, "both solvent-base or caustic products are commercially available that, when poured, brushed, or sprayed on painted exterior woodwork are capable of softening several layers of paint at a time so that the resulting "sludge"-which should be remembered is nothing less than the sequence of historic paint layers - can be removed with a putty knife. Detachable wood elements such as exterior shutters can also be "dip-stripped."

Solvent-base Strippers: The formulas tend to vary, but generally consist of combinations of organic solvents such as methylene chloride, isopropanol, toluol, xylol, and methanol; thickeners such as methyl cellulose; and various additives such as paraffin wax used to prevent the volatile solvents from evaporating before they have time to soak through multiple layers of paint. Thus, while some solvent-base strippers are quite thin and therefore unsuitable for use on vertical surfaces, others, called "semi-paste" strippers, are formulated for use on vertical surfaces or the underside of horizontal surfaces.

However, whether liquid or semi-paste, there are two important points to stress when using any solvent-base stripper: First, the vapors from the organic chemicals can be highly toxic if inhaled; skin contact is equally dangerous because the solvents can be absorbed; second, many solvent-base strippers are flammable. Even though application out-of-doors may somewhat mitigate health and safety hazards, a respirator with special filters for organic solvents is recommended and, of course, solvent-base strippers should never be used around open flames, lighted cigarettes, or with steel wool around electrical outlets.

Although appearing to be the simplest for exterior use, a particular type of solvent-base stripper needs to be mentioned here because it can actually cause the most problems. Known as "water-rinsable," such products have a high proportion of methylene chloride together with emulsifiers. Although the dissolved paint can be rinsed off with water with a minimum of scraping, this ultimately creates more of a problem in cleaning up and properly disposing of the sludge. In addition, these strippers can leave a gummy residue on the wood that requires removal with solvents. Finally, water-rinsable strippers tend to raise the grain of the wood more than regular strippers.

On balance, then, the regular strippers would seem to work just as well for exterior purposes and are perhaps even better from the standpoint of proper lead sludge disposal because they must be hand 'scraped as opposed to rinsed off (a coffeecan with a wire stretched across the top is one effective way to collect the sludge; when the putty knife is run across the wire, the sludge simply falls into the can. Then, when the can is filled, the wire is removed, the can capped, and the lead paint sludge disposed of according to local health regulations).

Ca ustic strippers: Until the advent of solvent-base strippers, caustic strippers were used exclusively when a chemical method was deemed appropriate for total paint removal prior to repainting or refinishing. Now, it is more difficult to find commercially prepared caustic solutions in hardware and paint stores for homeowner use with the exception of lye (caustic soda) because solvent-base strippers packaged in small quantities tend to dominate the market.

Most commercial dip stripping companies, however, continue to use variations of the caustic bath process because it is still the cheapest method available for removing paint. Generally, dip stripping should be left to professional companies because caustic solutions can dissolve skin and permanently damage eyes as well as present serious disposal problems in large quantities.

If exterior shutters or other detachable elements are being sent out for stripping in a caustic solution, it is wise to see samples of the company's finished work. While some companies do a first-rate job, others can leave a residue of paint in carvings and grooves. Wooden elements may also be soaked too long so that the wood grain is raised and roughened, requiring extensive hand sanding later. In addition, assurances should be given by these companies that caustic paint 
removers will be neutralized with a mild acid solution or at least thoroughly rinsed with water after dipping (a caustic residue makes the wood feel slippery). If this is not done, the lye residue will cause new paint to fail.

\section{Summary of Chemical Methods}

- Recommended, with extreme caution: Solvent-base strippers, caustic strippers.

- Applicable areas of buildings: decorative features, window muntins, doors, exterior shutters, columns, balusters, and railings.

- For use on: Class III Conditions.

- Health/Safety factors: Take precautions against inhaling toxic vapors; fire; eye damage; and chemical poisoning from skin contact. Dispose of lead residue properly

\section{General Paint Type Recommendations}

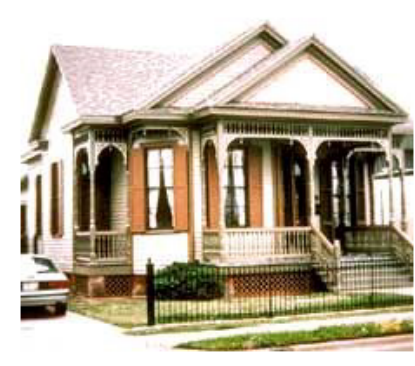

Decorative features were painted with a traditional oil-based paint as a part of the rehabilitation. Photo: NPS files.

Based on the assumption that the exterior wood has been painted with oil paint many times in the past and the existing top coat is therefore also an oil paint, it is recommended that for CLASS I and CLASS II paint surface conditions, a top coat of high quality oil paint be applied when repainting. The reason for recommending oil rather than latex paints is that a coat of latex paint applied directly over old oil paint is more apt to fail. The considerations are two ofold. First, because oil paints continue to harden with age, the old surface is sensitive to the added stress of shrinkage which occurs as a new coat of paint dries. Oil paints shrink less upon drying than la tex paints and thus do not have as great a tendency to pull the old paint loose. Second, when exterior oil paints age, the binder releases pigment particles, causing a chalky surface. Al though for best results, the chalk (or dirt, etc.) should always be cleaned off prior to repainting, a coat of new oil paint is more able to penetrate a chalky residue and adhere than is latex paint. Therefore, unless it is possible to thoroughly clean a heavily chalked surface, oil paintson balance-give better adhesion.

If however, a latex top coat is going to be applied over several layers of old oil paint, an oil primer should be applied first (the oil primer creates a flat, porous surface to which the latex can adhere). After the primer has thoroughly dried, a latex top coat may be applied. In the long run, changing paint types is more time consuming and expensive. An application of a new oil-type top coat on the old oil paint is, thus, the preferred course of action.

If CLASS III conditions have necessitated total paint removal, there are two options, both of which assure protection of the exterior wood: (1) an oil primer may be applied followed by an oil-type top coat, preferably by the same manufacturer; or (2) an oil primer may be applied followed by a latex top coat, again using the same brand of paint. It should also be noted that primers were never intended to withstand the effects of weathering; therefore, the top coat should be applied as soon as possible after the primer has dried.

\section{Summary and References}

The recommendations outlined in this Brief are cautious because at present there is no comple tely safe and effective method of removing old paint from exterior woodwork. This has necessarily eliminated descriptions of several me thods still in a developmental or experimental stage, which can therefore neither be recommended nor precluded from future recommendation. Wth the ever-increasing number of buildings being rehabilitated, however, paint removal technology should be stimulated and, in consequence, existing methods refined and new methods developed which will respect both the historic wood and the health and safety of the operator.

\section{Acknowledgements}

Special thanks go to Baird M. Smith, AIA (formerly Chief, Preservation Technology Branch, TPS) for providing general direction in the development of the manuscript. In addition, the following individuals are to be thanked for their contributions as technical experts in the field: Royal T. Brown, National Paint and Coatings Association, Washington, D.C.; Dr. Judith E. Selwyn, Preservation Technology Associates, Boston, Massachusetts; and Dennis R. Vacca, Pratt \& Lambert Co., Carlstadt, New Jersey. Finally, thanks go to several National Park Service staff members whose valuable comments were incorporated into the text and who contributed to the production of the brief: James A. Caufield, Anne E. Grimmer, Jean E. Travers, David G. Batte, Sharon C. Park, AIA, Charles E. Fisher III, Sara K. Blumenthal, and Martha A. Gutrick. The peeling paint photo used as the logo is provided, courtesy, John Leeke, Preservation Consultant, 2001, and is copyrighted. 
Preservation Services (TPS), National Park Service prepares standards, guidelines, and other educational materials on responsible historic preservation treatments for a broad public.

September 1982

\section{Reading List}

Batcheler, Penelope Hartshorne, "Paint Color Research and Restoration." Technfcal Leaffet 15. Nashville: American Association for State and Local History (undated).

"Danger: Restoration May Be Hazardous to Your Health." The Old House Journal. Vol. 4, No. 5 (May 1976), pp. 911. Gola, Edward F. "Avoiding Mistakes in Exterior Painting." The Old House Journal. Vol. 4, No. 6 (June 1976), pp. 1, 45

"How to Assure a Satisfactory Paint Job." Scfentfffc Section: Cfrcular 784. Washington, DC: National Paint, Varnish and Lacquer Association (undated).

Labine, Clem. "Selecting the Best Exterior Paint." The Odd House Journal. Vol. 4, No. 7 (July 1976), pp. 1, 1011.

Morton, W. Brown III and Hume, Gary L. The Secretary of the Interror's Standards for Historic Preservation Projects with Gudelines for Applying the Standards. Washington, DC: Department of Interior, 1979.

Paint Problem Solver. St. Louis: National Decorating Products Association, 1980.

"Special Issue: Exterior Painting." The Old House Journal. Vol. 4, No. 4 (April 1981), pp. 7194.

Thorsen, John W. "Hazardous Waste: What is it? How to Handle it." Professfonal Decorating and Coating Action. Vol. 43, No. 4 (September 1981), pp. 45. 


\title{
4.3.2 Replacing deteriorated woodwork, ${ }^{12} 2017$ (GSA.gov).
}

\section{GSA U.S. General Services Administration}

\section{Replacing Deteriorated Woodwork}

\author{
Procedure code: \\ $640015 S$ \\ Source: \\ National Capitol Region Specifications \\ Division: \\ Concrete \\ Section: \\ Architectural Woodwork \\ Last Modified: \\ $02 / 24 / 2017$
}

\section{PART 1---GENERAL}

\subsection{SUMMARY}

A. This procedure includes guidance on wood restoration work including repairing existing woodwork by removing damaged or deteriorated material and replacing with new to match existing.

B. See 01100-07-S for general project guidelines to be reviewed along with this procedure. These guidelines cover the following sections:

1. Safety Precautions

2. Historic Structures Precautions

3. Submittals

4. Quality Assurance

5. Delivery, Storage and Handling

6. Project/Site Conditions

7. Sequencing and Scheduling

8. General Protection (Surface and Surrounding)

These guidelines should be reviewed prior to performing this procedure and should be followed, when applicable, along with recommendations from the Regional Historic Preservation Officer (RHPO).

\subsection{REFERENCES}

A. AWI Quality Standard: Comply with applicable requirements of Architectural Woodwork Standards, published by the Architectural Woodwork Institute (www.AWInet.net), except as otherwise indicated.

\subsection{SYSTEM DESCRIPTION}

A. Performance Requirements: Submit written program for each phase of restoration process including protection of surrounding materials on building during operations. Describe in detail materials, methods and equipment to be used for each phase of restoration work.

\footnotetext{
12 Retrieved from: https://www.gsa.gov/real-estate/historic-preservation/historic-preservation-policytools/preservation-tools-resources/technical-documents.
} 


\subsection{QUALITY ASSURANCE}

A. Mock-Ups: Prior to start of wood restoration work, prepare the following sample panels in building where directed by RHPO. Obtain RHPO's acceptance of visual qualities before proceeding with the work. Retain acceptable panels in undisturbed condition, suitably marked, during construction as a standard for judging completed work.

1. Wood Repair: Prepare sample panels for each type of woodwork indicated to be patched, resurfaced, modified or replaced. Prepare mock-up panels on existing woodwork to demonstrate quality of materials and workmanship.

\subsection{PROJECT/SITE CONDITIONS}

A. Existing Conditions:

1. Installer shall advise Contractor of temperature and humidity requirements for woodwork installation areas. Do not install woodwork until required temperature and relative humidity have been stabilized and will be maintained in installation areas.

2. Maintain temperature and humidity in installation area as required to maintain moisture content of installed woodwork within a 1.0 percent tolerance of optimum moisture content, from date of installation through remainder of construction period. The fabricator of woodwork shall determine optimum moisture content and required temperature and humidity conditions.

3. Determine that surfaces to which finishes are to be applied are even, smooth, sound, clean, dry and free from defects affecting proper application. Correct or report defective surfaces to Contracting Officer.

\section{PART 2---PRODUCTS}

\subsection{MATERIALS}

A. New or Replacement Materials:

1. Wood Moisture Content: Provide kiln-dried lumber with an average moisture content range of $6 \%$ to $11 \%$ for interior work. Maintain temperature and relative humidity during fabrication, storage and finishing operations so that moisture content values for woodwork at time of installation do not exceed the above range.

2. Replacement Wood: Match species, grade, grain pattern, and other special characteristics of existing woodwork.

B. Clean, soft cloths

\section{PART 3---EXECUTION}

\subsection{PREPARATION}

A. Surface Preparation:

1. Condition woodwork to average prevailing humidity conditions in installation areas prior to installing.

2. Back prime woodwork on all surfaces which will be concealed with one coat of wood primer. Schedule delivery to allow time for application and drying of Back prime coat before installation of woodwork.

3. Remove miscellaneous hardware, nails, etc., from all existing woodwork as required to provide a first class installation of new or replacement woodwork.

4. Prior to installation of new architectural woodwork, examine shop fabricated work for completion, and complete work as required, including back priming and removal of packing.

\subsection{ERECTION, INSTALLATION, APPLICATION}

A. Carefully remove at locations indicated any damaged or deteriorated woodwork. Unless indicated otherwise, replace the entire length of the existing damaged piece to the next butt joint.

B. For partial replacement of existing pieces, use a neat, well-fitted level cut with grain aligned in transparent finished wood.

C. Install new pieces as described below:

1. Install the work plumb, level, true and straight with no distortions. Shim as required using concealed shims. 
2. Cut to fit unless specified to be shop-fabricated or shop-cut to exact size. Where woodwork abuts other finished work, scribe and cut for accurate fit. Before making cutouts, drill pilot holes at corners.

3. Standing and Running Trim: Install with minimum number of joints possible, using full-length pieces (from maximum length of lumber available) to the greatest extent possible. Stagger joints in adjacent and related members. Cope at returns, miter at corners, and comply with Quality Standards for joinery.

4. Anchor woodwork to anchors or blocking built-in or directly attached to substrates. Secure to grounds, stripping and blocking with countersunk, concealed fasteners and blind nailing as required for a complete installation. Except where prefinished matching fasteners heads are required, use fine finishing nails for exposed nailing, countersunk and filled flush with woodwork, and matching final finish where transparent finish is indicated.

D. Finish replacement woodwork to match adjacent woodwork surfaces. See 06400-05-R and 06400-10-R for guidance.

\subsection{ADJUSTING/CLEANING}

A. Upon completion of this work, all floors, walls, and other adjacent surfaces that are stained, marred, or otherwise damaged by work under this section shall be cleaned and repaired and all work and the adjacent areas shall be left in a clean and perfect condition.

B. All completed work shall be adequately protected from damage by subsequent building operations and effects of weather. Protection shall be by methods recommended by the manufacturer of installed materials and as approved by the RHPO.

C. Repair damaged and defective woodwork wherever possible to eliminate defects functionally and visually; where not possible to repair properly, replace woodwork. Adjust joinery for uniform appearance.

D. Clean woodwork: Dust and damp wipe woodwork with a soft cloth dampened in clean water; dry rub with soft cloth to maintain the polish, rubbing along the grain of the wood.

E. Stain and Spot Removal:

1. Stains may be cleaned by prompt damp wiping with cloth dampened in clear water or rubbing with cloth dampened in solvent. Dry the wood with a soft cloth.

2. White spots may be removed by rubbing them with a small amount of linseed oil. 


\title{
4.3.3 Patching woodwork, ${ }^{13} 2017$ (GSA.gov).
}

GSA U.S. General Services Administration

Patching Cracks And Holes In Woodwork

\author{
Procedure code: \\ $640016 \mathrm{~S}$ \\ Source: \\ National Capitol Region Specifications \\ Division: \\ Concrete \\ Section: \\ Architectural Woodwork \\ Last Modified: \\ 06/01/2017
}

PART 1---GENERAL

\subsection{SUMMARY}

A. This procedure includes guidance on patching cracks and small holes in woodwork

B. See 01100-07-S for general project guidelines to be reviewed along with this procedure. These guidelines cover the following sections:

1. Safety Precautions

2. Historic Structures Precautions

3. Submittals

4. Quality Assurance

5. Delivery, Storage and Handling

6. Project/Site Conditions

7. Sequencing and Scheduling

8. General Protection (Surface and Surrounding)

These guidelines should be reviewed prior to performing this procedure and should be followed, when applicable, along with recommendations from the Regional Historic Preservation Officer (RHPO).

\subsection{REFERENCES}

A. AWI Quality Standard: Comply with applicable requirements of the latest edition of "Architectural Woodwork Standards", published by the Architectural Woodwork Institute (www.AWInet.org), except as otherwise indicated.

\subsection{SYSTEM DESCRIPTION}

A. Performance Requirements: Submit written program for each phase of restoration process including protection of surrounding materials on building during operations. Describe in detail materials, methods and equipment to be used for each phase of restoration work.

13 Retrieved from: https://www.gsa.gov/real-estate/historic-preservation/historic-preservation-policytools/preservation-tools-resources/technical-documents. 


\subsection{QUALITY ASSURANCE}

A. Mock-Ups: Prior to start of wood restoration work, prepare the following sample panels in building where directed by RHPO. Obtain RHPO's acceptance of visual qualities before proceeding with the work. Retain acceptable panels in undisturbed condition, suitably marked, during construction as a standard for judging completed work.

1. Wood Repair: Prepare sample panels for each type of woodwork indicated to be patched, resurfaced, modified or replaced. Prepare mock-up panels on existing woodwork to demonstrate quality of materials and workmanship.

\subsection{PROJECT/SITE CONDITIONS}

A. Existing Conditions:

1. Installer shall advise Contractor of temperature and humidity requirements for woodwork installation areas. Do not install woodwork until required temperature and relative humidity have been stabilized and will be maintained in installation areas.

2. Maintain temperature and humidity in installation area as required to maintain moisture content of installed woodwork within a 1.0 percent tolerance of optimum moisture content, from date of installation through remainder of construction period. The fabricator of woodwork shall determine optimum moisture content and required temperature and humidity conditions.

3. Determine that surfaces to which finishes are to be applied are even, smooth, sound, clean, dry and free from defects affecting proper application. Correct or report defective surfaces to Contracting Officer.

\section{PART 2---PRODUCTS}

\subsection{MATERIALS}

A. Patching Materials:

1. Wood Filler: Standard filler manufactured specifically for restorative patching of woodwork. a. Tint filler to match existing woodwork.

2. Sandpaper: No. 3/0 or No. 5/0 garnet paper.

B. Replacement Wood: Match species, grade, grain pattern, and other special characteristics of existing woodwork.

\section{PART 3---EXECUTION}

\subsection{ERECTION, INSTALLATION, APPLICATION}

A. Remove all minor surface imperfections such as scratches, dents, etc., by rubbing surface with a fine grit sandpaper.

B. Patch all holes and cracks in woodwork up to $1 / 16$ (one sixteenth) inch across with wood filler tinted to match existing wood.

C. Carefully hand rub filled area with a fine grit sandpaper to match surface characteristics of adjacent woodwork.

D. Touch-up patch during finishing so that color and other appearance characteristics of filled area match the $>$ finish of adjacent woodwork. See 06400-05-R, 06400-06-R, and 06400-10-R for guidance on refinishing.

E. Patch holes and cracks in woodwork $1 / 4$ (one quarter) inch and greater across and woodwork damaged from hardware changes with wood plugs or wood patches or as directed by manufacturer.

F. Rout out hole or crack woodwork to receive plus or patch materials. Veneer-type patches shall be comparable to the thickness of the surrounding intact veneer and historically $1 / 16$ (one sixteenth) to $1 / 20$ (one twenthieth) inch thick, referring to the thickness of the veneer.

G. All repair plugs and patches in wood with a transparent finish shall have grain aligned.

\subsection{ADJUSTING/CLEANING}

A. Upon completion of this work, all floors, walls, and other adjacent surfaces that are stained, marred, or otherwise damaged by work under this section shall be cleaned and repaired and all work and the adjacent areas shall be left in a clean and perfect condition. 
B. All completed work shall be adequately protected from damage by subsequent building operations and effects of weather. Protection shall be by methods recommended by the manufacturer of installed materials and as approved by the RHPO.

C. Repair damaged and defective woodwork wherever possible to eliminate defects functionally and visually; where not possible to repair properly, replace woodwork. Adjust joinery for uniform appearance.

D. Clean woodwork: Dust and damp wipe woodwork with a soft cloth dampened in clean water; dry rub with soft cloth to maintain the polish, rubbing along the grain of the wood.

E. Stain and Spot Removal:

1. Stains may be cleaned by prompt damp wiping with cloth dampened in clear water or rubbing with cloth dampened in solvent. Dry the wood with a soft cloth.

2. White spots may be removed by rubbing them with a small amount of linseed oil. 


\section{Stage III - Windows}

The wood and glass in old windows are generally very sturdy, repairable, and these features can last for generations if properly protected and maintained. Windows should be considered significant to a building if they are original, reflect the original design intent for the building, reflect period or regional styles or building practices, reflect changes to the building resulting from major periods or events, and/or they exemplify exceptional craftsmanship or design. Once an evaluation of the significance of the wood window has been completed, it is possible to proceed with planning appropriate treatments, beginning with an investigation of the physical condition of the window (excerpt from Myers 1981 - Preservation Brief \#9).

\subsection{Immediate concerns for windows}

Many factors such as poor design, moisture, vandalism, insect attack, and lack of maintenance can contribute to window deterioration, but moisture is the primary contributing factor in wood window decay. The sill should be examined to ensure that it slopes downward away from the building and allows water to drain off. Generally, the actions necessary to return a window to "like new" condition fall into three categories: (1) routine maintenance procedures, (2) structural stabilization, and (3) parts replacement.

Whatever the causes of deterioration, a careful analysis of the deterioration, supplemented by testing, is vital to the success of any wood window repair project. Repair of historic wood windows may consist of either patching the historic material or filling in with new material worked to match the historic material. If replacement is necessary, duplication of historic materials and detailing should be as exact as possible to ensure a repair that is functionally and aesthetically acceptable.

Wood window elements are evaluated as follows:

- All windows should be inspected to see if water is entering around the edges of the frame and, if so, the joints or seams should be caulked to eliminate this danger.

- Glazing putty should be checked for cracked, loose, or missing sections. 
- Paint failure is seen in paint blistering, cracking, flaking, and peeling, and these conditions usually identify points of water penetration, moisture saturation, and potential deterioration.

All of the windows on Bldg. 550 are original eight-over-eight wood-sash double-hung windows from 1942. It is rare that over time the windows were not replaced. It is important to consider the size, shape, window frame, the pane, the opening mechanisms, and the hardware when evaluating windows. All components of the windows in Bldg. 550 are original. There are two types of windows found on Bldg. 550 were of standard design used throughout the construction of the WWII buildings at Camp McCoy in 1942. One type was labeled "Type A" on the original drawings, and are, according to the Standard Windows Details sheet, Plan 800-151, 4'-6" tall by nearly 3'5 " wide (see Figures 54 and 55). This window type was used on the north and south elevations of Bldg. 550 on the living area/squad room section of the building. A second type "Type B" measured 5'-6" tall by 3'-5" wide as used on the north and south elevations of the apparatus room (see Figure 56). A standard wood frame mesh-screen accompanied each window. According to HABS documentation, the window trim, frame, and muntins and screen frame would have been painted a light-yellow color. 
Figure 54. Plan 800-151, Standard Window Details, 1942.

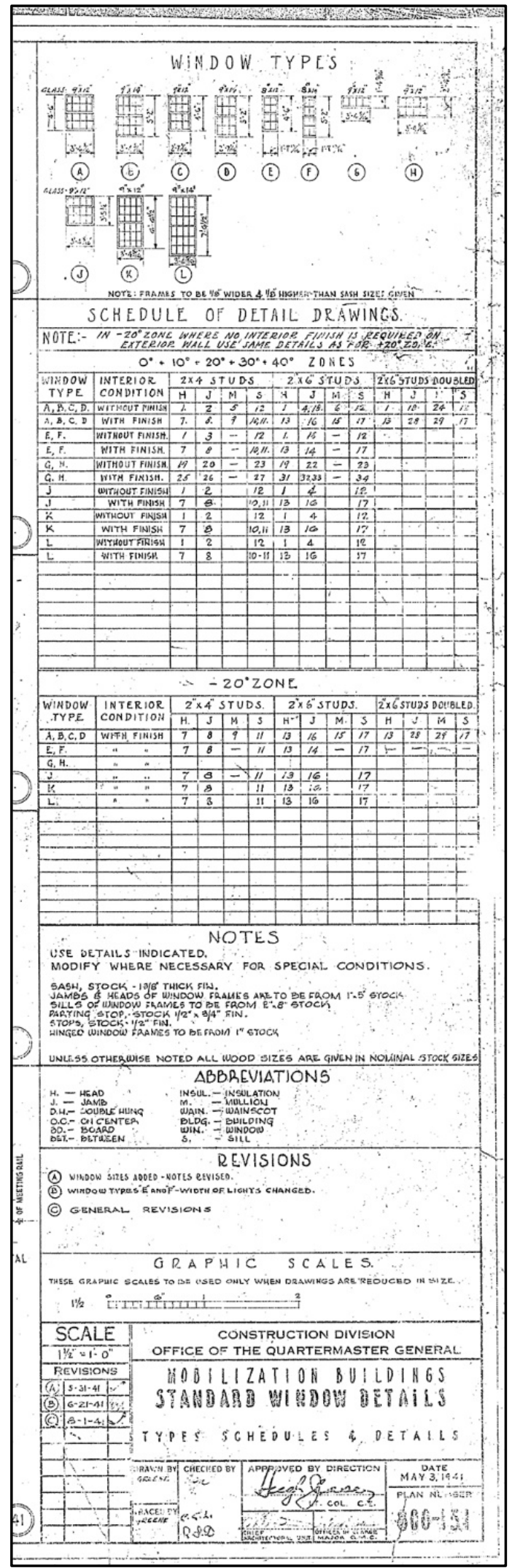

Source: Fort McCoy DPW. 
Figure 55. Close-up detail taken from original plans for standard window Type A, 1942.

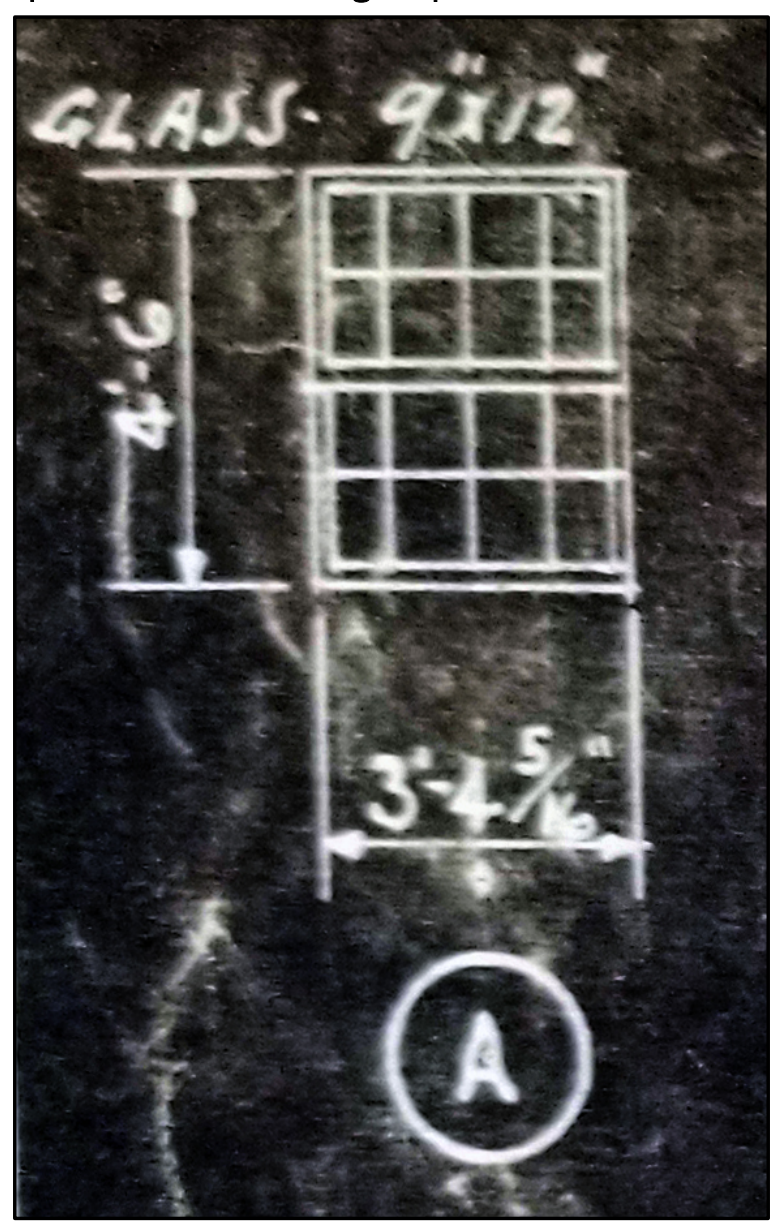

Source: Fort McCoy DPW. 
Figure 56. Close-up detail taken from original plans for standard window Type B, 1942.

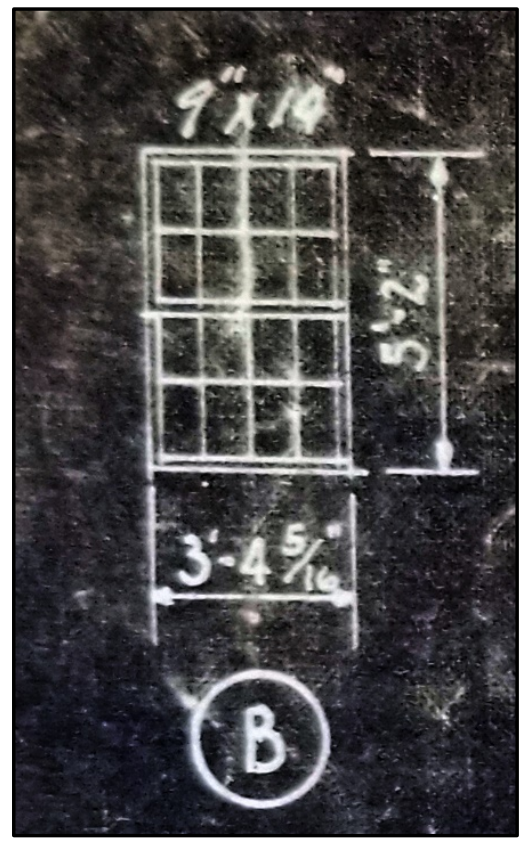

Source: Fort McCoy DPW.

Looking at (Figure 57) below, "Type A" is regularly used in the living area/squad room section of the building, while "Type B" was used in the apparatus room (Figure 58) shows window B used in the apparatus room.

Figure 57. Looking at a portion of "Framing Elevation C" from the original plans, 1942.

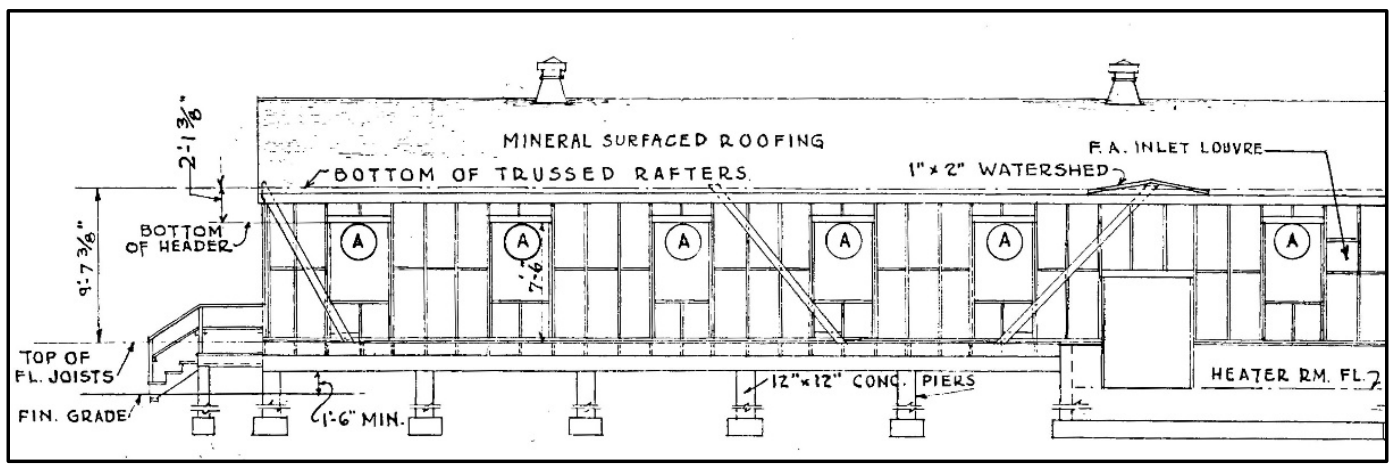

Source: Fort McCoy DPW. 
Figure 58. Looking at Portion of “Framing Elevation B" from the original plans, 1942.

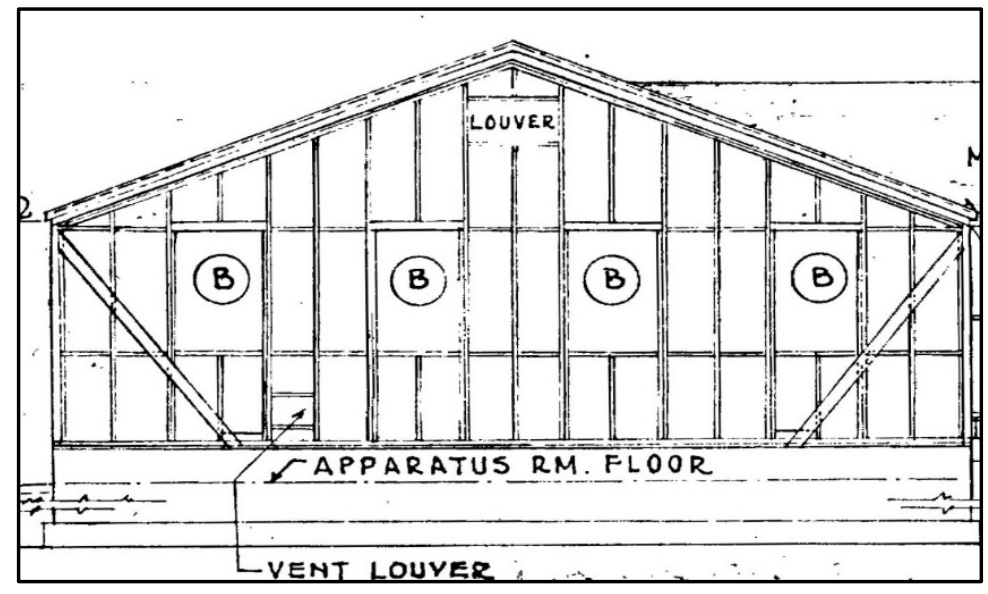

Source: Fort McCoy DPW.

\subsection{Exterior wood windows}

There were 23 original standard design windows on Bldg. 550; 14 "Type A" (see Figure 59) and nine "Type B" (see Figure 60). The windows consist of typical wood muntins with glass placed in a rabbet on the outside and held in place with glazing.

In 2018, the condition of the majority of the windows was considered poor.

Approximately half of the original wooden screen had been removed in 2018. There is obvious paint failure on the frame, muntins, and trim of the window and the frame of the screen. Much of the window glazing is missing. The screen appears to be loose and possibly rusted in the higher regions. The screen should be maintained in the same manner as the wood window.

All windows are original to the period of significance and need to be retained and repaired. If removal and replacement is absolutely necessary, they will need to be replaced in-kind. 
Figure 59. Original eight-over-eight wood-sash double-hung window with wooden screen on Bldg. 550, 2018.

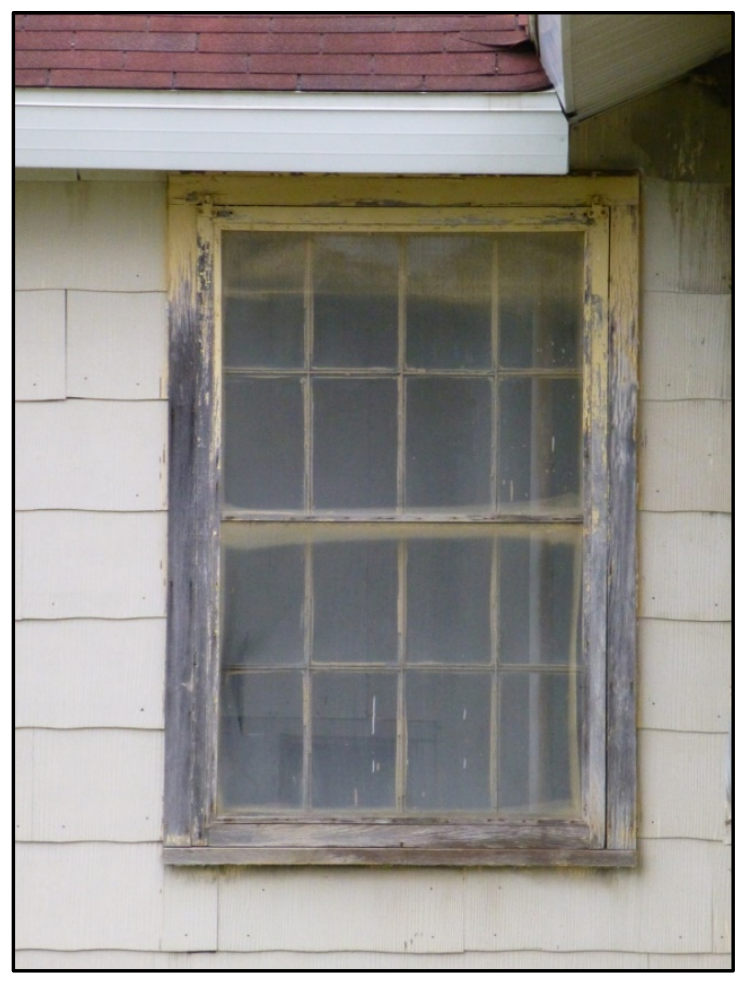

Figure 60. Example of a “Type A” window (left) and “Type B” window (right), 2018.

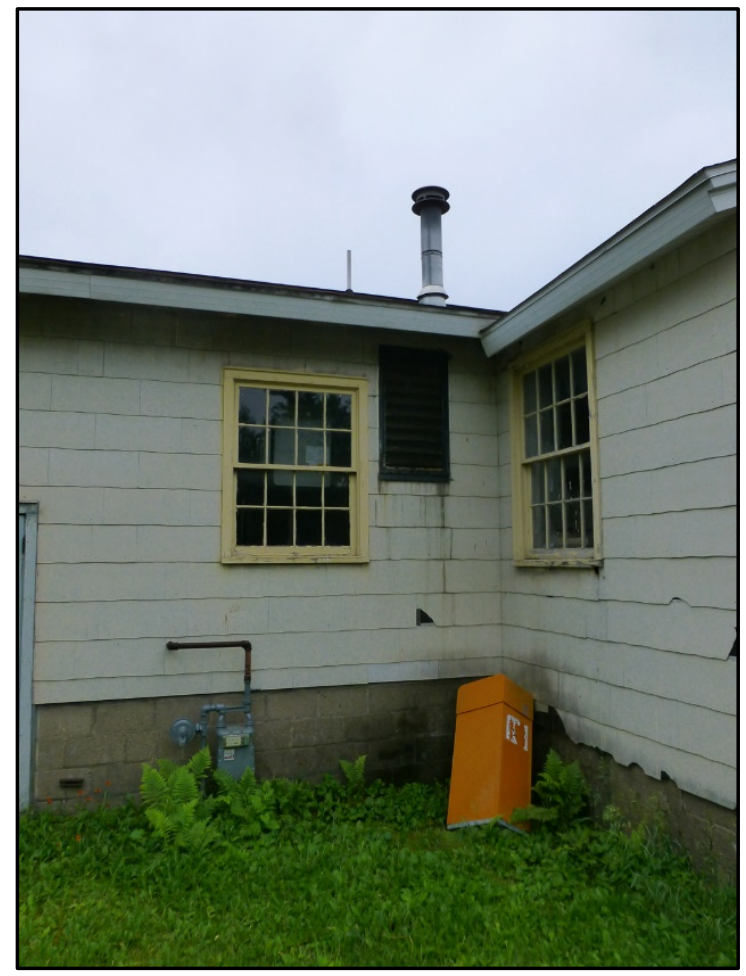




\subsection{Interior wood windows}

The interior window trim is a simple flat wood board casings. Due to the installment of inner plastic coverings and the lack of replacing the glazing, many of the windows are in poor shape (see Figure 61).

Figure 61. Looking at interior wood window on south side of Bldg. 550, 2018.

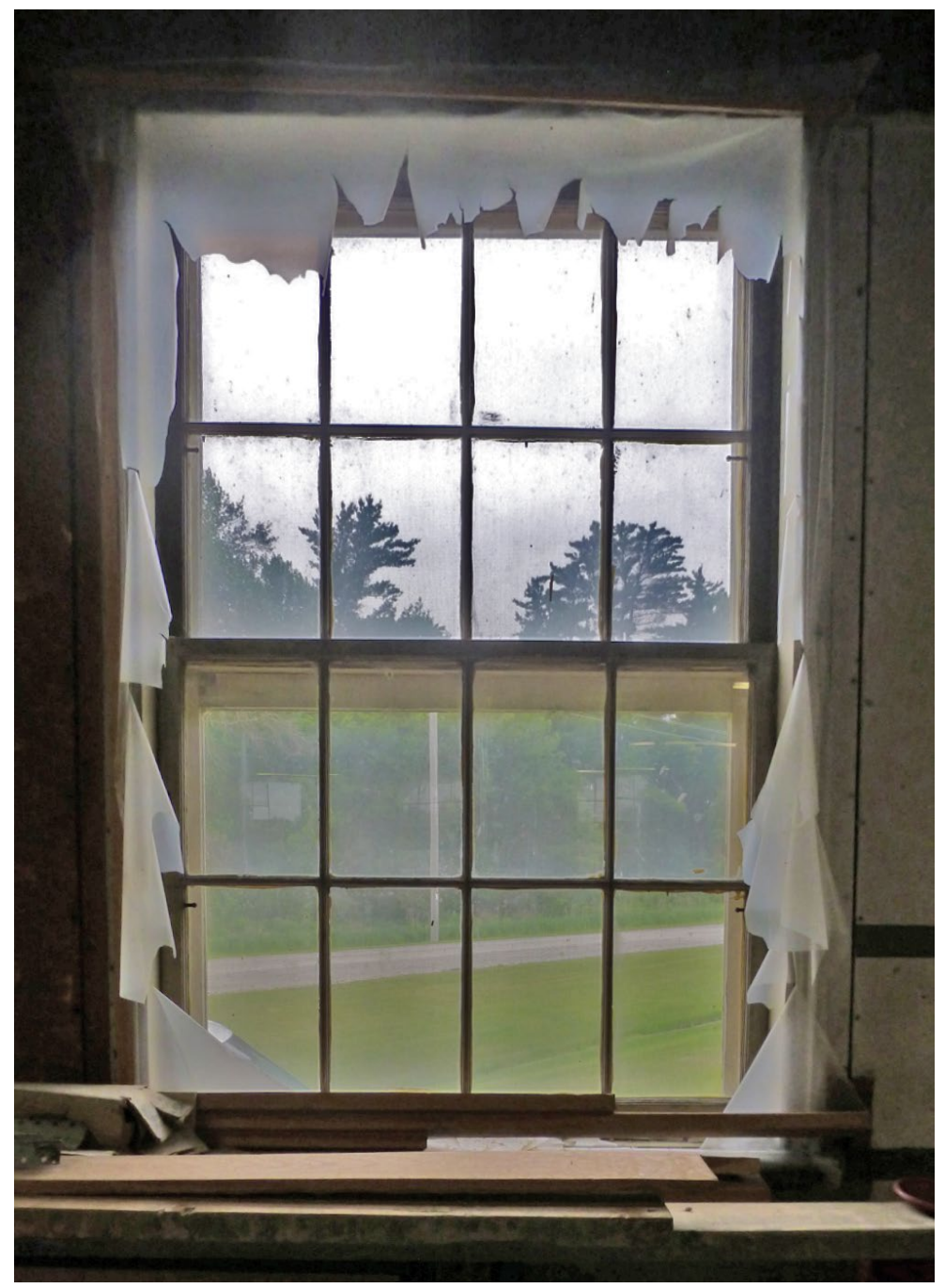

\subsection{Treatment measures}

Bldg. 550 has all of its original wooden windows from 1942. The information provided below gives a background and repair/restoration methods that can be used on wooden windows similar to Bldg. 550 . 


\subsubsection{Preservation Brief 9: ${ }^{14}$ The repair of historic wooden windows, 1981 (NPS.gov).}

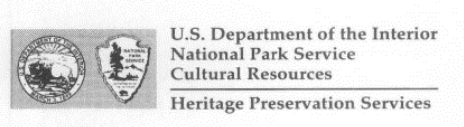

National Park Service
Cultural Resources

Heritage Preservation Services

\section{Preservation Briefs: 9}

The Repair of

Historic Wooden Windows

John H. Myers

The windows on many historic buildings are an important aspect of the architectural character of those buildings. Their design, craftsmanship, or other qualities may make them worthy of preservation. This is self-evident for ornamental windows, but it can be equally true for warehouses or factories where the windows may be the most dominant visual element of an otherwise plain building (see figure 1). Evaluating the significance of these windows and planning for their repair or replacement can be a complex process involving both objective and subjective considerations. The Secretary of the Interior's Standards for Rehabilitation, and the accompanying guidelines, call for respecting the significance of original materials and features, repairing and retaining them wherever possible, and when necessary, replacing them in kind. This Brief is based on the issues of significance and repair which are implicit in the standards, but the primary emphasis is on the technical issues of planning for the repair of windows including evaluation of their physical condition, techniques of repair, and design considerations when replacement is necessary.

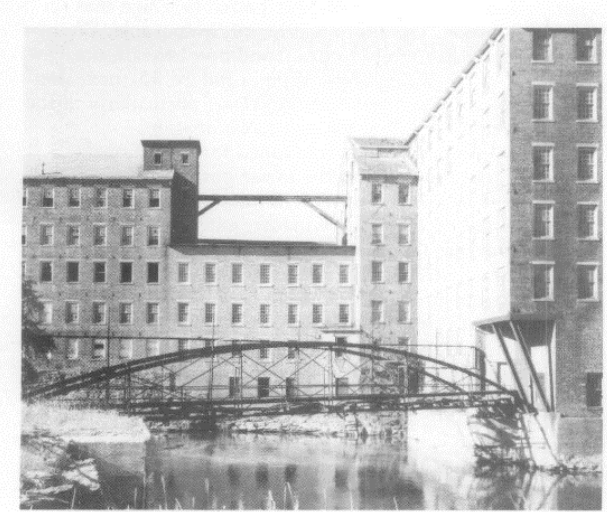

Figure 1. Windows are frequently important visual focal points, especially on simple facades such as this mill building. Replacement of the multipane windows here with larger panes could dramatically change the appearance of the building. The areas of missing windows convey the impression of such a change. Photo: John T. Lowe
Much of the technical section presents repair techniques as an instructional guide for the do-it-yourselfer. The information will be useful, however, for the architect, contractor, or developer on large-scale projects. It presents a methodology for approaching the evaluation and repair of existing windows, and considerations for replacement, from which the professional can develop alternatives and specify appropriate materials and procedures.

Architectural or Historical Significance

Evaluating the architectural or historical significance of windows is the first step in planning for window treatments, and a general understanding of the function and history of windows is vital to making a proper evaluation. As a part of this evaluation, one must consider four basic window functions: admitting light to the interior spaces, providing fresh air and ventilation to the interior, providing a visual link to the outside world, and enhancing the appearance of a building. No single factor can be disregarded when planning window treatments; for example, attempting to conserve energy by closing up or reducing the size of window openings may result in the use of more energy by increasing electric lighting loads use of more energy by increasing electric
and decreasing passive solar heat gains.

and decreasing passive solar heat gains.
Historically, the first windows in early American houses were casement windows; that is, they were hinged at the side and opened outward. In the beginning of the eighteenth century single- and double-hung windows were introduced. Subsequently many styles of these vertical sliding sash windows have come to be associated with specific building periods or architectural styles, and this is an important consideration in determining the significance of windows, especially on a local or regional basis. Siteof windows, especially on a local or regional basis. Site-
specific, regionally oriented architectural comparisons specific, regionally oriented architectural comparisons
should be made to determine the significance of windows in question. Although such comparisons may focus on specific window types and their details, the ultimate determination of significance should be made within the context of the whole building, wherein the windows are one architectural element (see figure 2).

After all of the factors have been evaluated, windows should be considered significant to a building if they: 1) are original, 2) reflect the original design intent for the building, 3) reflect period or regional styles or building practices, 4) reflect changes to the building resulting from major periods or events, or 5) are examples of exceptional craftsmanship or design. Once this evaluation of significance has been completed, it is possible to pro- 


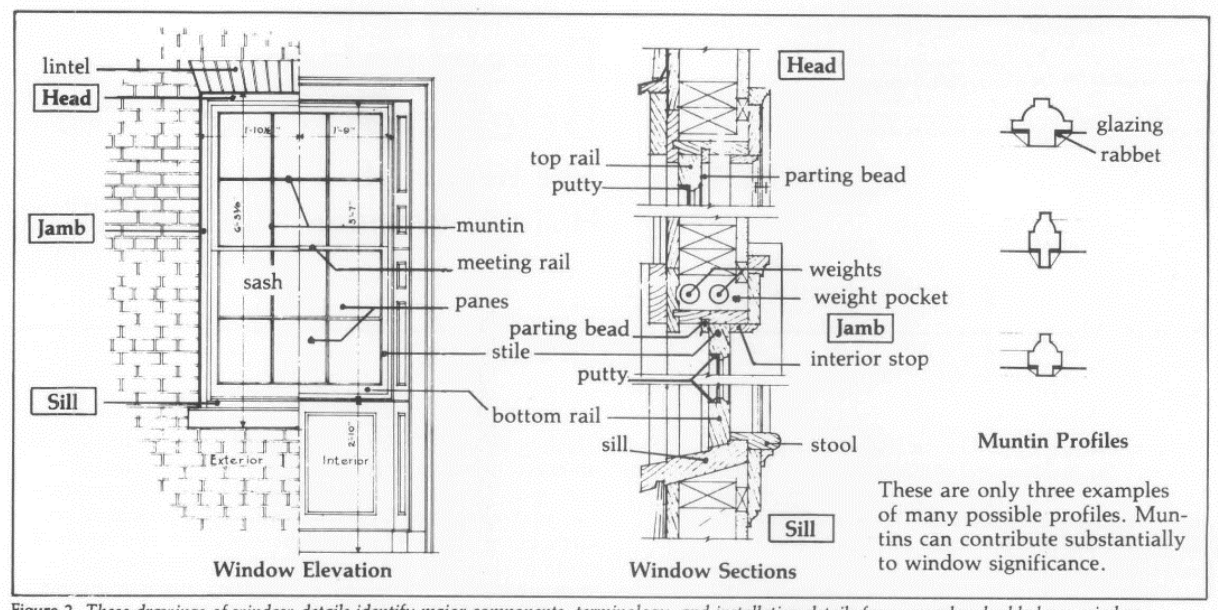

Figure 2. These drawings of window details identify major components, terminology, and installation details for a wooden double-hung window.

ceed with planning appropriate treatments, beginning with an investigation of the physical condition of the windows.

Physical Evaluation

The key to successful planning for window treatments is a careful evaluation of existing physical conditions on a unit-by-unit basis. A graphic or photographic system may be devised to record existing conditions and illustrate the scope of any necessary repairs. Another effective tool is a window schedule which lists all of the parts of each window unit. Spaces by each part allow notes on existing conditions and repair instructions. When such a schedule is completed, it indicates the precise tasks to be performed in the repair of each unit and becomes a part of the specifications. In any evaluation, one should note at a minimum, 1) window location, 2) condition of the paint, 3 ) condition of the frame and sill, 4) condition of the sash (rails, stiles and muntins), 5) glazing problems, 6) hard(rails, stiles and muntins), 5) glazing problems, 6) hard-
ware, and 7) the overall condition of the window (exware, and 7) the overall condition
cellent, fair, poor, and so forth).

Many factors such as poor design, moisture, vandalism, insect attack, and lack of maintenance can contribute to window deterioration, but moisture is the primary contributing factor in wooden window decay. All window units should be inspected to see if water is entering around the edges of the frame and, if so, the joints or seams should be caulked to eliminate this danger. The glazing putty should be checked for cracked, loose, or missing sections which allow water to saturate the wood, especially at the joints. The back putty on the interior side of the pane should also be inspected, because it creates a sea which prevents condensation from running down into the joinery. The sill should be examined to insure that it slopes downward away from the building and allows water to drain off. In addition, it may be advisable to cut a dripline along the underside of the sill. This almost invisible treatment will insure proper water run-off, particu- larly if the bottom of the sill is flat. Any conditions, including poor original design, which permit water to come in contact with the wood or to puddle on the sill must be corrected as they contribute to deterioration of the window.

One clue to the location of areas of excessive moisture is the condition of the paint; therefore, each window should be examined for areas of paint failure. Since excessive moisture is detrimental to the paint bond, areas of paint blistering, cracking, flaking, and peeling usually identify points of water penetration, moisture saturation, and potential deterioration. Failure of the paint should not, however, be mistakenly interpreted as a sign that the wood is in poor condition and hence, irreparable. Wood is frequently in sound physical condition beneath unsightly paint. After noting areas of paint failure, the next step is to inspect the condition of the wood, particularly at the points identified during the paint examination.

Each window should be examined for operational soundness beginning with the lower portions of the frame and sash. Exterior rainwater and interior condensation can flow downward along the window, entering and collecting at points where the flow is blocked. The sill, joints between the sill and jamb, corners of the bottom rails and muntin joints are typical points where water collects and deterioration begins (see figure 3 ). The operation of the window (continuous opening and closing over the years and seasonal temperature changes) weakens the joints, causing movement and slight separation. This process makes the joints more vulnerable to water which is readily absorbed into the end-grain of the wood. If severe deterioration exists in these areas, it will usually be apparent on visual inspection, but other less severely deteriorated areas of the wood may be tested by two traditional methods using a small ice pick.

An ice pick or an awl may be used to test wood for soundness. The technique is simply to jab the pick into a wetted wood surface at an angle and pry up a small sec- 


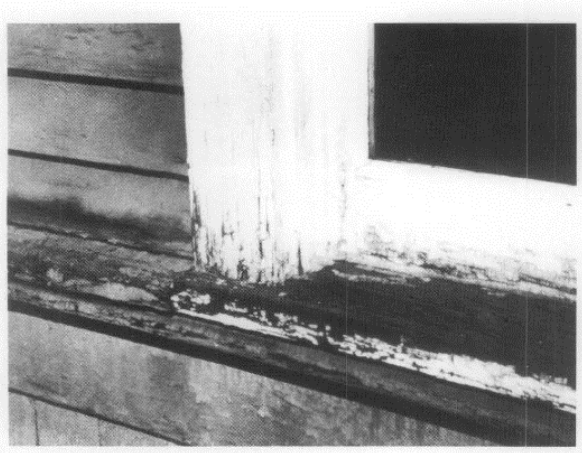

Figure 3. Deterioration of poorly maintained windows usually begins on horizontal surfaces and at joints where water can collect and saturate the wood. The problem areas are clearly indicated by paint failure due to

tion of the wood. Sound wood will separate in long fibrous splinters, but decayed wood will lift up in short irregular pieces due to the breakdown of fiber strength. Another method of testing for soundness consists of pushing a sharp object into the wood, perpendicular to the surface. If deterioration has begun from the hidden side of a member and the core is badly decayed, the visible surface may appear to be sound wood. Pressure on the probe can force it through an apparently sound skin to penetrate deeply into decayed wood. This technique is especially useful for checking sills where visual access to the underside is restricted.

Following the inspection and analysis of the results, the scope of the necessary repairs will be evident and a plan for the rehabilitation can be formulated. Generally the ac tions necessary to return a window to "like new" condition will fall into three broad categories: 1) routine maintenance procedures, 2) structural stabilization, and 3) parts replacement. These categories will be discussed in the following sections and will be referred to respectively as Repair Class I, Repair Class II, and Repair Class III. Each successive repair class represents an increasing level of difficulty, expense, and work time. Note that most of the points mentioned in Repair Class I are routine main tenance items and should be provided in a regular maintenance program for any building. The neglect of these routine items can contribute to many common window problems.

Before undertaking any of the repairs mentioned in the following sections all sources of moisture penetration

should be identified and eliminated, and all existing decay fungi destroyed in order to arrest the deterioration process. Many commercially available fungicides and wood preservatives are toxic, so it is extremely important to follow the manufacturer's recommendations for application, and store all chemical materials away from children and animals. After fungicidal and preservative treatment the windows may be stabilized, retained, and restored with every expectation for a long service life.

Repair Class I: Routine Maintenance

Repairs to wooden windows are usually labor intensive and relatively uncomplicated. On small scale projects this allows the do-it-yourselfer to save money by repairing all or part of the windows. On larger projects it presents the opportunity for time and money which might otherwise be spent on the removal and replacement of existing wise be spent on the removal and replacement of existing
windows, to be spent on repairs, subsequently saving all or part of the material cost of new window units. Regardless of the actual costs, or who performs the work, the evaluation process described earlier will provide the knowledge from which to specify an appropriate work program, establish the work element priorities, and idenprogram, establish the work element priorities,
tify the level of skill needed by the labor force.

The routine maintenance required to upgrade a window to "like new" condition normally includes the following steps: 1) some degree of interior and exterior paint removal, 2) removal and repair of sash (including reglazing where necessary), 3) repairs to the frame, 4) weatherstripping and reinstallation of the sash, and 5) repainting. These operations are illustrated for a typical double-hung wooden window (see figures $4 a-f$ ), but they may be adapted to other window types and styles as applicable.

Historic windows have usually acquired many layers of paint over time. Removal of excess layers or peeling and flaking paint will facilitate operation of the window and restore the clarity of the original detailing. Some degree of paint removal is also necessary as a first step in the proper surface preparation for subsequent refinishing (if paint er surfacin the onset of the paint removal). There are several safe and the onset of the paint removal). There are several safe
effective techniques for removing paint from wood, depending on the amount of paint to be removed. Severa techniques such as scraping, chemical stripping, and the use of a hot air gun are discussed in "Preservation Briefs: 10 Paint Removal from Historic Woodwork" (see Additional Reading section at end).

Paint removal should begin on the interior frames, being careful to remove the paint from the interior stop and the parting bead, particularly along the seam where these stops meet the jamb. This can be accomplished by running a utility knife along the length of the seam, breaking the paint bond. It will then be much easier to remove the stop, the parting bead and the sash. The interior stop may be initially loosened from the sash side to avoid visible scarring of the wood and then gradually pried loose using a pair of putty knives, working up and down the stop in small increments (see figure $4 \mathrm{~b}$ ). With the stop removed, the lower or interior sash may be withdrawn. The sash cords should be detached from the sides of the sash and their ends may be pinned with a nail or tied in a knot to prevent them from falling into the weight pocket.

Removal of the upper sash on double-hung units is similar but the parting bead which holds it in place is set into a groove in the center of the stile and is thinner and more delicate than the interior stop. After removing any paint along the seam, the parting bead should be carefully pried out and worked free in the same manner as the interior stop. The upper sash can be removed in the same manner as the lower one and both sash taken to a convenient work area (in order to remove the sash the interior stop and parting bead need only be removed from one side of the window). Window openings can be covered with polyethylene sheets or plywood sheathing while the sash are out for repair.

The sash can be stripped of paint using appropriate techniques, but if any heat treatment is used (see figure $4 \mathrm{c})$, the glass should be removed or protected from the sudden temperature change which can cause breakage. An 


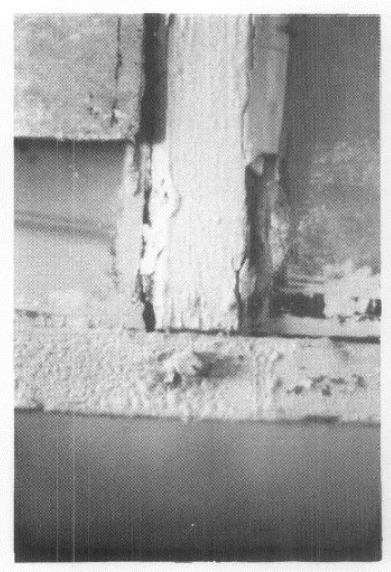

Figure 4a. The following series of photographs of the repair of a historic double-hung window use
unit which is structurally sound but has many layers of paint, some cracked and missing putty, slight separation at the joints, broken sash cords, and one cracked pane. Photo: John H. Myers

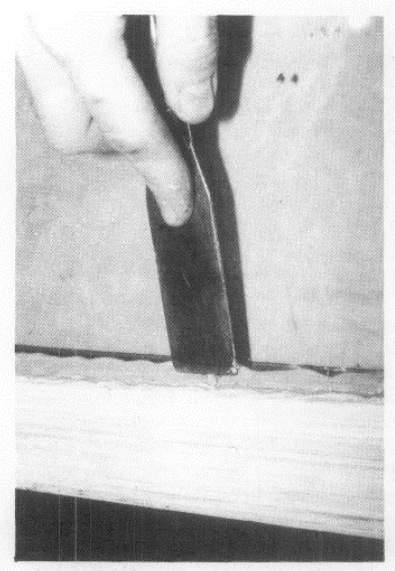

Figure $4 \mathrm{~d}$. Reglazing or replacement of the putty requires that the existing putty be removed

manually, the glazing points be extracted, the
glass removed, and the back putty scraped out. T glass removed, and the back putty scraped out. To
reglaze, a bed of putty is laid around the perimeter reglaze, a bed of putty is laid around the perime of the raboet, the pane is pressed into place, glazing points are inserted to hold the pane around the edge of the glass. Phy is beveled Myers

4

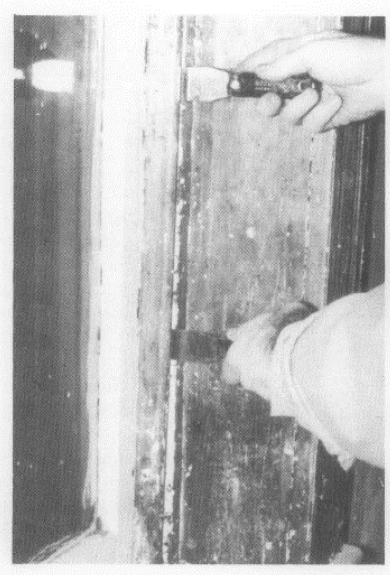

Figure $4 \mathrm{~b}$. After removing paint from the seam between the interior stop and the jamb, the stop can be pried out and gradually worked loose using a pair of putty knives as shown. To avoid visible the stop pried loose initially from the outer side. Photo: John H. Myers

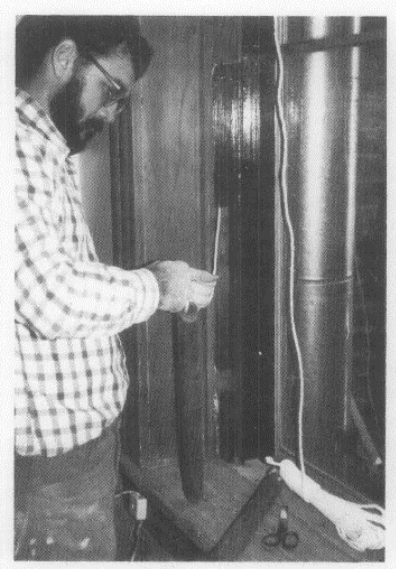

Figure 4e. A common repair is the replacement of broken sash cords with new cords (shown) or wi chains. The weight pocket is often accessible

through a removable plate in the jamb, or by removing the interior trim. Photo: John H. Myers

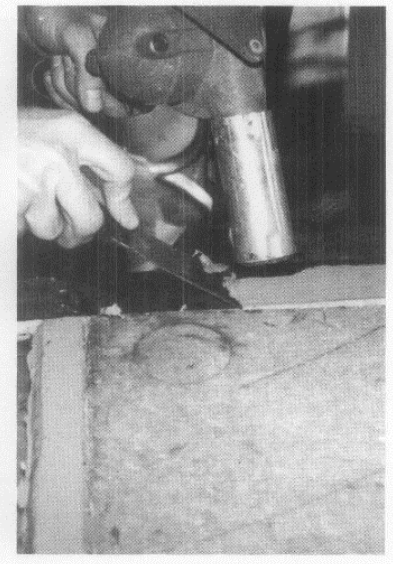

Figure 4c. Sash can be removed and repaired in a convenient work area. Paint is being removed from sheet protects the glass from sudden temperature change. Photo: John H. Myers

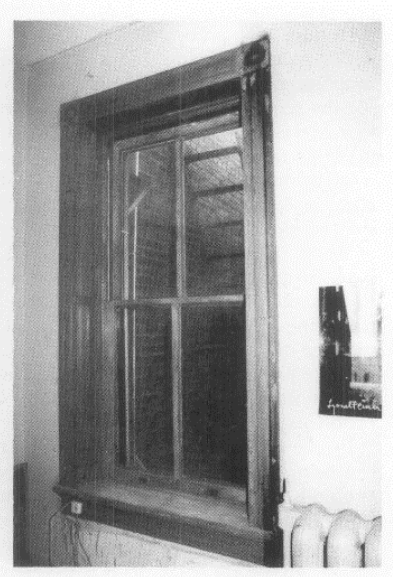

Figure 4f. Following the relatively simple repairs, the window is weathertight, like new in

appearance, and serviceable for many years to come. Both the historic material and the detailing and craftsmanship of this original window have 
overlay of aluminum foil on gypsum board or asbestos can protect the glass from such rapid temperature change. It is important to protect the glass because it may be historic and often adds character to the window. Deteriorated putty should be removed manually, taking care not to damage the wood along the rabbet. If the glass is to be removed, the glazing points which hold the glass in place can be extracted and the panes numbered glass in place can be extracted and the panes numbered
and removed for cleaning and reuse in the same openand removed for cleaning and reuse in the same open-
ings. With the glass panes out, the remaining putty can be removed and the sash can be sanded, patched, and primed with a preservative primer. Hardened putty in the rabbets may be softened by heating with a soldering iron at the point of removal. Putty remaining on the glass may be softened by soaking the panes in linseed oil, and then removed with less risk of breaking the glass. Before reinstalling the glass, a bead of glazing compound or linseed oil putty should be laid around the rabbet to cushion and seal the glass. Glazing compound should only be used on wood which has been brushed with linseed oil and primed with an oil based primer or paint. The pane is then pressed into place and the glazing points are pushed into the wood around the perimeter of the pane (see figure 4d). The final glazing compound or putty is applied and beveled to complete the seal. The sash can be refinished as desired on the inside and painted on the outside as soon as a "skin" has formed on the putty, usually in 2 or 3 days. Exterior paint should cover the beveled glazing compound or putty and lap over onto the glass slightly to complete a weathertight seal. After the proper curing times have elapsed for paint and putty, the sash will be ready for reinstallation.

While the sash are out of the frame, the condition of the wood in the jamb and sill can be evaluated. Repair and refinishing of the frame may proceed concurrently with repairs to the sash, taking advantage of the curing times for the paints and putty used on the sash. One of the most common work items is the replacement of the sash cords with new rope cords or with chains (see figure $4 \mathrm{e})$. The weight pocket is frequently accessible through a door on the face of the frame near the sill, but if no door exists, the trim on the interior face may be removed for access. Sash weights may be increased for easier window operation by elderly or handicapped persons. Additiona repairs to the frame and sash may include consolidation or replacement of deteriorated wood. Techniques for these repairs are discussed in the following sections.

The operations just discussed summarize the efforts necessary to restore a window with minor deterioration to "like new" condition (see figure 4f). The techniques can be applied by an unskilled person with minimal training and experience. To demonstrate the practicality of this approach, and photograph it, a Technical Preservation Services staff member repaired a wooden double-hung, two over two window which had been in service over ninety years. The wood was structurally sound but the window had one broken pane, many layers of paint, broken sash cords and inadequate, worn-out weatherstripping. The staff member found that the frame could be stripped of paint and the sash removed quite easily. Paint, putty and glass removal required about one hour for each sash, and the reglazing of both sash was accomplished in about one hour. Weatherstripping of the sash and frame, replacement of the sash cords and reinstallation of the sash, parting bead, and stop required an hour and a half. These times refer only to individual operations; the entire proc- ess took several days due to the drying and curing times for putty, primer, and paint, however, work on other window units could have been in progress during these lag times.

\section{Repair Class II: Stabilization}

The preceding description of a window repair job focused on a unit which was operationally sound. Many windows will show some additional degree of physical deterioration, especially in the vulnerable areas mentioned earlier but even badly damaged windows can be repaired using simple processes. Partially decayed wood can be waterproofed, patched, built-up, or consolidated and then painted to achieve a sound condition, good appearance, and greatly extended life. Three techniques for repairing and greatly extended life. Three techniques for repairing partially decayed or weathered wood are discussed in this section, and all three can be accomplished using products available at most hardware stores.

One established technique for repairing wood which is split, checked or shows signs of rot, is to: 1) dry the wood, 2) treat decayed areas with a fungicide, 3) waterproof with two or three applications of boiled linseed oil (applications every 24 hours), 4) fill cracks and holes with putty, and 5) after a "skin" forms on the putty, paint the surface. Care should be taken with the use of fungicide which is toxic. Follow the manufacturers' directions and use only on areas which will be painted. When using any technique of building up or patching a flat surface, the finished surface should be sloped slightly to carry water away from the window and not allow it to puddle. Caulking of the joints between the sill and the jamb will help reduce further water penetration.

When sills or other members exhibit surface weathering they may also be built-up using wood putties or homemade mixtures such as sawdust and resorcinol glue, or whiting and varnish. These mixtures can be built up in successive layers, then sanded, primed, and painted. The same caution about proper slope for flat surfaces applies to this technique.

Wood may also be strengthened and stabilized by consolidation, using semi-rigid epoxies which saturate the porous decayed wood and then harden. The surface of the consolidated wood can then be filled with a semi-rigid epoxy patching compound, sanded and painted (see figure 5). Epoxy patching compounds can be used to build up

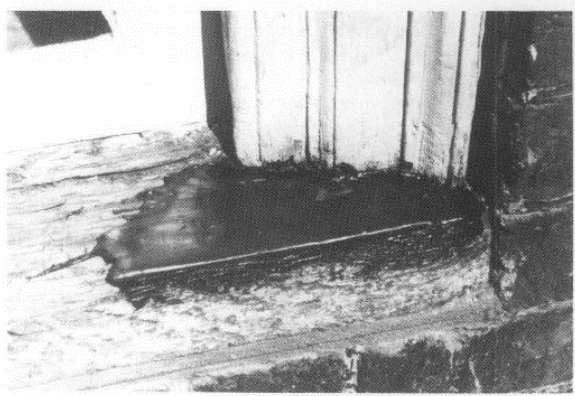

Figure 5. This illustrates a two-part epoxy patching compound used to fill the surface of a weathered sill and rebuild the missing edge. When the epoxy cures, it can be sanded smooth and painted to achieve a durable and
waterproof repair. Photo: John H. Myers 
missing sections or decayed ends of members. Profiles can be duplicated using hand molds, which are created by pressing a ball of patching compound over a sound section of the profile which has been rubbed with butcher's wax. This can be a very efficient technique where there are many typical repairs to be done. Technical Preservation Services has published Epoxies for Wood Repairs in Historic Buildings (see Additional Reading section at end), which discusses the theory and techniques of epoxy repairs. The process has been widely used and proven in marine applications; and proprietary products are available at hardware and marine supply stores. Although epoxy materials may be comparatively expensive, they hold the promise of being among the most durable and long lasting materials available for wood repair.

Any of the three techniques discussed can stabilize and restore the appearance of the window unit. There are times, however, when the degree of deterioration is so advanced that stabilization is impractical, and the only way to retain some of the original fabric is to replace damaged parts.

Repair Class III: Splices and Parts Replacement When parts of the frame or sash are so badly deteriorated that they cannot be stabilized there are methods which permit the retention of some of the existing or original fabric. These methods involve replacing the deteriorated parts with new matching pieces, or splicing new wood into existing members. The techniques require more skill and are more expensive than any of the previously discussed alternatives. It is necessary to remove the sash and/or the affected parts of the frame and have a carpenter or woodworking mill reproduce the damaged or missing parts. Most millwork firms can duplicate parts, such as muntins, bottom rails, or sills, which can then be incorporated into the existing window, but it may be necessary to shop around because there are several factors controlling the practicality of this approach. Some woodworking mills do not like to repair old sash because nails or other foreign objects in the sash can damage expensive knives (which cost far more than their profits on small repair jobs); others do not have cutting knives to duplicate muntin profiles. Some firms prefer to concentrate on larger jobs with more profit potential, and some may not have a craftsman who can duplicate the parts. A little searching should locate a firm which will do the job, and at a reasonable price. If such a firm does not exist locally, there are firms which undertake this kind of repair and ship nationwide. It is possible, however, for the advanced do-it-yourselfer or craftsman with a table saw to duplicate moulding profiles using techniques discussed by Gordie Whittington in "Simplified Methods for Reproducing Wood Mouldings," Bulletin of the Association for Preservation Technology, Vol. III, No. 4, 1971, or illustrated more recently in The Old House. Time-Life Books, Alexandria, Virginia, 1979.

The repairs discussed in this section involve window frames which may be in very deteriorated condition, possibly requiring removal; therefore, caution is in order. The actual construction of wooden window frames and sash is not complicated. Pegged mortise and tenon units can be disassembled easily, if the units are out of the building. The installation or connection of some frames to the surrounding structure, especially masonry walls, can complicate the work immeasurably, and may even require dismantling of the wall. It may be useful, therefore, to take the following approach to frame repair: 1) conduct regular maintenance of sound frames to achieve the longest life possible, 2) make necessary repairs in place wherever possible, using stabilization and splicing techniques, and 3) if removal is necessary, thoroughly investigate the structural detailing and seek appropriate professional consultation.

Another alternative may be considered if parts replacement is required, and that is sash replacement. If extensive replacement of parts is necessary and the job becomes prohibitively expensive it may be more practical to purchase new sash which can be installed into the existing frames. Such sash are available as exact custom reproductions, reasonable facsimiles (custom windows with similar profiles), and contemporary wooden sash which are similar in appearance. There are companies which still manufacture high quality wooden sash which would duplicate most historic sash. A few calls to local building suppliers may provide a source of appropriate replacement sash, but if not, check with local historical associations, the state historic preservation office, or preservation related magazines and supply catalogs for information.

If a rehabilitation project has a large number of windows such as a commercial building or an industrial complex, there may be less of a problem arriving at a solution. Once the evaluation of the windows is completed and the scope of the work is known, there may be a potential economy of scale. Woodworking mills may be interested in the work from a large project; new sash in volume may be considerably less expensive per unit; crews can be assembled and trained on site to perform all of the window repairs; and a few extensive repairs can be absorbed (without undue burden) into the total budget for a large number of sound windows. While it may be expensive for the average historic home owner to pay seventy dollars or more for a mill to grind a custom knife to duplicate four or five bad muntins, that cost becomes negligible on large commercial projects which may have several hundred windows.

Most windows should not require the extensive repairs discussed in this section. The ones which do are usually in buildings which have been abandoned for long periods or have totally lacked maintenance for years. It is necessary to thoroughly investigate the alternatives for windows which do require extensive repairs to arrive at a solution which retains historic significance and is also economically feasible. Even for projects requiring repairs identified in this section, if the percentage of parts replacement per window is low, or the number of windows requiring repair is small, repair can still be a cost effective solution.

\section{Weatherization}

A window which is repaired should be made as energy efficient as possible by the use of appropriate weatherstripping to reduce air infiltration. A wide variety of products are available to assist in this task. Felt may be fastened to the top, bottom, and meeting rails, but may have the disadvantage of absorbing and holding moisture, particularly at the bottom rail. Rolled vinyl strips may also be tacked into place in appropriate locations to reduce infiltration. Metal strips or new plastic spring strips may be used on the rails and, if space permits, in 
the channels between the sash and jamb. Weatherstripping is a historic treatment, but old weatherstripping (felt) is not likely to perform very satisfactorily. Appropriate contemporary weatherstripping should be considered an integral part of the repair process for windows. The use of sash locks installed on the meeting rail will insure that the sash are kept tightly closed so that the weatherstripping will function more effectively to reduce infiltration. Although such locks will not always be historically accurate, they will usually be viewed as an acceptable contem porary modification in the interest of improved thermal performance.

Many styles of storm windows are available to improve the thermal performance of existing windows. The use of exterior storm windows should be investigated whenever feasible because they are thermally efficient, cost-effective, reversible, and allow the retention of original windows (see "Preservation Briefs: $3^{\prime \prime}$ ). Storm window frames may be made of wood, aluminum, vinyl, or plastic; however, the use of unfinished aluminum storms should be avoided. The visual impact of storms may be minimized by selecting colors which match existing trim color. Arched top storms are available for windows with special shapes. Although interior storm windows appear to offer an attractive option for achieving double glazing with minimal visual impact, the potential for damaging condensation problems must be addressed. Moisture which becomes trapped between the layers of glazing can condense on the colder, outer prime window, potentially leading to deterioration. The correct approach to using in terior storms is to create a seal on the interior storm while allowing some ventilation around the prime window. In actual practice, the creation of such a durable, airtight seal is difficult.

\section{Window Replacement}

Although the retention of original or existing windows is always desirable and this Brief is intended to encourage that goal, there is a point when the condition of a window may clearly indicate replacement. The decision process for selecting replacement windows should not begin with a survey of contemporary window products which are available as replacements, but should begin with a look at the windows which are being replaced. Attempt to understand the contribution of the window(s) to the appearance of the facade including: 1) the pattern of the openings and their size; 2) proportions of the frame and sash; 3) configuration of window panes; 4) muntin profiles; 5) type of wood; 6) paint color; 7) characteristics of the glass; and 8) associated details such as arched tops, hoods, or other decorative elements. Develop an understanding of how the window reflects the period, style, or regional characteristics of the building, or represents technological development.

Armed with an awareness of the significance of the existing window, begin to search for a replacement which retains as much of the character of the historic window as possible. There are many sources of suitable new windows. Continue looking until an acceptable replacemen can be found. Check building supply firms, local woodworking mills, carpenters, preservation oriented magazines, or catalogs or suppliers of old building materials, for product information. Local historical associations and state historic preservation offices may be good sources of information on products which have been used successfully in preservation projects.

Consider energy efficiency as one of the factors for

replacements, but do not let it dominate the issue. Energy conservation is no excuse for the wholesale destruction of historic windows which can be made thermally efficient by historically and aesthetically acceptable means. In fact, a historic wooden window with a high quality storm window added should thermally outperform a new doubleglazed metal window which does not have thermal

breaks (insulation between the inner and outer frames intended to break the path of heat flow). This occurs because the wood has far better insulating value than the metal, and in addition many historic windows have high ratios of wood to glass, thus reducing the area of highest heat transfer. One measure of heat transfer is the U-value, the number of Btu's per hour transferred through a square foot of material. When comparing thermal performance, the lower the U-value the better the performance. According to ASHRAE 1977 Fundamentals, the U-values for single glazed wooden windows range from 0.88 to 0.99 . The addition of a storm window should reduce these figures to a range of 0.44 to 0.49 . A non-thermal break, double-glazed metal window has a U-value of about 0.6.

\section{Conclusion}

Technical Preservation Services recommends the retention and repair of original windows whenever possible. We believe that the repair and weatherization of existing wooden windows is more practical than most people realize, and that many windows are unfortunately replaced because of a lack of awareness of techniques for evaluation, repair, and weatherization. Wooden windows which are repaired and properly maintained will have greatly extended service lives while contributing to the historic character of the building. Thus, an important element of a building's significance will have been preserved for the future.

\section{Additional Reading}

ASHRAE Handbook-1977 Fundamentals. New York: American Society of Heating, Refrigerating and Air-conditioning Engineers, 1978 of Heating,
(chapter 26).

Ferro, Maximillian. Preservation: Present Pathway to Fall River's Future. Fall River, Massachusetts: City of Fall River, 1979 (chapter 7)

"Fixing Double-Hung Windows." Old House Journal (no. 12, 1979): 135.

Look, David W. "Preservation Briefs: 10 Paint Removal from Historic Woodwork." Washington, DC: Technical Preservation Services, U.S. Department of the Interior, forthcoming.

Morrison, Hugh. Early American Architecture. New York: Oxford University Press, 1952.

Phillips, Morgan, and Selwyn, Judith. Epoxies for Wood Repairs in Historic Buildings. Washington, DC: Technical Preservation SerStock No. 024-016-00095-1), 1978.

Rehab Right. Oakland, California: City of Oakland Planning Department, 1978 (pp. 78-83).

"Sealing Leaky Windows." Old House Journal (no. 1, 1973): 5 .

Smith, Baird M. "Preservation Briefs: 3 Conserving Energy in Historic Buildings." Washington, DC: Technical Preservation Services, U.S. Department of the Interior, 1978. 


\title{
5.4.2 Rehabilitating wood windows, ${ }^{15} 2017$ (GSA.gov).
}

GSA U.S. General Services Administration

\section{Rehabilitating Wood Windows}

\author{
Procedure code: \\ 8610015 \\ Source: \\ The Old Custom House/New York, Ny - Gsa/Pbs, 1991 \\ Division: \\ Doors and Windows \\ Section: \\ Wood Windows \\ Last Modified: \\ 07/05/2017
}

\section{PART 1---GENERAL}

\subsection{SUMMARY}

A. This procedure includes guidance for the rehabilitation of wood windows. Outlined are the steps one might go through to complete repairs. Each step is cross-referenced to one or more procedures which covers the particular problem. The cross-referenced procedures should be reviewed prior to beginning window repairs and should be followed along with recommendations from the Regional Historic Preservation Officer (RHPO).

B. The steps in the repair of deteriorated sash include but are not limited to the following:

1. Examination, survey and condition assessment of windows.

2. Removal of existing sash, trim, etc.

3. Repair of deteriorated wood through the use of epoxies, dutchmen and/or the replacement with new wood to match the existing appearance.

4. Painting/refinishing sash and trim.

5. Installation of repaired sash.

C. See 01100-07-S for general project guidelines to be reviewed along with this procedure. These guidelines cover the following sections:

1. Safety Precautions

2. Historic Structures Precautions

3. Submittals

4. Quality Assurance

5. Delivery, Storage and Handling

6. Project/Site Conditions

7. Sequencing and Scheduling

8. General Protection (Surface and Surrounding) These guidelines should be reviewed before performing this procedure and should be followed, when applicable, along with recommendations from the RHPO.

D. For general information on the repair of wood windows see 08610-01-S, "Preservation Briefs: 9 - The Repair of Historic Wooden Windows."

15 Retrieved from: https://www.gsa.gov/real-estate/historic-preservation/hioric-preservation-policytools/preservation-tools-resources/technical-documents. 


\subsection{SUBMITTALS}

A. Shop drawings for each type of window, including $1 / 4$-inch scale wall elevations, typical unit elevations at 3/4inch scale, glazing details, and full-size details of typical composite members, include window rehabilitation, wood and hardware replacement, reglazing details and weatherstripping.

B. The RHPO reserves the right to require additional samples that show fabrication techniques and construction and design of hardware and accessories.

\subsection{SEQUENCING AND SCHEDULING}

A. Rehabilitation of windows shall be completed before doing any interior restoration/rehabilitation work to insure weather-tight integrity of interior spaces.

\section{PART 2---PRODUCTS}

\subsection{MATERIALS}

NOTE: See specific procedures for materials and equipment requirements, and their manufacturers and sources.

\section{PART 3---EXECUTION}

\subsection{EXAMINATION}

A. Conduct a window-by-window survey to determine existing conditions and identify the specific work needs of each window.

B. For each window type, the survey should include color photographs which show design details for comparison to new work, and existing conditions.

1. Full frame views, both interior and exterior.

2. Close-up views of typical details, both interior and exterior.

\subsection{ERECTION, INSTALLATION, APPLICATION}

A. Carefully remove window stops, sash and trim as required. Remove only those features which cannot be repaired on- site. All disassembled parts should be indelibly marked or stamped on hidden parts so they can be returned to their exact location.

1. See 06440-03-R, "Closing Open Joints in Wood Wall Ornament"

B. Replace rotted window sills as required.

1. See 08610-04-R, "Replacing a Wood Window Sill"

C. Repair, replace, or rebuild all rotted or deteriorated wood features. These can include but are not limited to stiles, rails, muntins, joints, frame, trim. New work shall match existing profiles or shapes in every respect and shall be flush with existing adjacent surfaces.

1. See 06300-01-R, "Epoxy Repair for Deterioration and Decay in Wooden Members"

2. See 08611-01-R, "Sealing Leaky Wood Double-Hung Windows"

3. See 06440-01-R, "Repairing Cracks and Checks in Wood Wall Ornament

4. See 06440-03-R, "Closing Open Joints in Wood Wall Ornament"

5. See 06440-04-R, "Repairing Scratches, Gouges and Dents in Wood Wall Ornament"

6. See 09560-03-R, "Dutchman Repair of Wood Floor Boards"

D. Remove paint from both interior (where applicable) and exterior surfaces.

1. See 06400-07-R, "Chemically Removing Paint From Wood Features"

2. See 06400-02-S, "Supplemental Guidelines for Removing Paint from Interior and Exterior Wood Surfaces"

3. See 06400-09-R, "Removing Paint From Wood Features Using Thermal Methods"

E. Remove all deteriorated glazing putty and broken glass. Replace glass and reglaze with a flexible elastomeric glazing compound. Clean the existing historic glass. See 08800-01-R, "Replacing Broken Glass in Wood and Metal Windows"

F. Reinstall windows. Inspect pull chains and weights at all double hung windows and adjust, clean or replace as required to ensure proper operation. Lubricate all working parts to assure smooth operation.

1. See 08760-01-R, "Repairing Double-Hung Window Sash Weights and Cords/Chains" 


\title{
5.4.3 General guidelines for wood windows, ${ }^{16} 2016$ (GSA.gov).
}

GSA U.S. General Services Administration

\section{General Guidelines For The Fabrication And Installation Of Wood Windows}

\author{
Procedure code: \\ 8610025 \\ Source: \\ Federal Building/Uspo, Spokane, Wa - Gsa/Pbs \\ Division: \\ Doors and Windows \\ Section: \\ Wood Windows \\ Last Modified: \\ 07/13/2016
}

PREFACE: Windows are an important design element contributing to a building s architectural character. For historic buildings, state historic preservation officers generally apply the Secretary of Interior Standards for Rehabilitation which require that original material be repaired and retained where possible. Condition surveys are typically required to determine if windows are too deteriorated to save, before replacements will be considered. Replacement windows should match the color, size and configuration of the original windows and the material.

PART 1---GENERAL

1.01 SUMMARY

A. This procedure includes general information relating to the fabrication and installation of wood windows andincludes the following window types:

1. Double-Hung Window Units.

2. Casement Window Units.

3. Decorative Window Units

4. Hopper Window Units.

5. Non-Operative (Fixed) Window Units.

6. Single-Hung Window Units.

B. See 01100-07-S for general project guidelines to be reviewed along with this procedure. These guidelines cover the following sections:

1. Safety Precautions

2. Historic Structures Precautions

3. Submittals

4. Quality Assurance

5. Delivery, Storage and Handling

6. Project/Site Conditions

7. Sequencing and Scheduling

16 Retrieved from: https://www.gsa.gov/real-estate/historic-preservation/historic-preservation-policytools/preservation-tools-resources/technical-documents. 
8. General Protection (Surface and Surrounding)

9. These guidelines should be reviewed prior to performing this procedure and should be followed, when applicable, along with recommendations from the Regional Historic Preservation Officer (RHPO).

\subsection{REFERENCES}

A. American Society for Testing and Materials (ASTM), 100 Barr Drive, West Conshohocken, PA 19428 $610-832-9585$ 610-832-9555.

B. American National Standards Institute, Inc. (ANSI), 1430 Broadway, New York, NY 10018.

Flat Glass Marketing Association (FGMA), National Wood Window and Door Association (NWWDA),

\subsection{SYSTEM DESCRIPTION}

A. Performance Grade Classification: Provide wood windows that comply with requirements of NWWDA I.S. 2 for performance grade 40.

B. Performance Grade Classification: Provide wood windows that comply with requirements of NWWDA I.S. 2 for performance grade 60 .

C. Standards: Performance requirements for structural performance, air infiltration, and water penetration for wood windows are those specified in NWWDA I.S."Industry Standard for Wood Window Units".

D. Testing: Manufacturer's stock units of each grade of required wood window shall have been tested by a recognized testing laboratory or agency, in accordance with ASTM E330 for structural performance. Test samples shall comply with requirements in NWWDA I.S. 2 for test sample sizes and methods.

E. Performance Requirements (Grade 60 Windows): Each required window unit shall comply with the following performance requirements:

A. Air Infiltration: Not more than $0.10 \mathrm{cfm}$ per sq. $\mathrm{ft}$. of overall frame area at an inward test pressure of 1.57 lbfper sq. ft.

B. Water Penetration: No water penetration as defined in the test method at an inward test pressure of $6.24 \mathrm{lbf}$ per sq. ft.

c. Structural Performance: No glass breakage, damage to hardware, or permanent deformation that would impair operation of the unit or residual deflection greater than 0.4 percent of the span at a positive (inward) and negative (outward) test pressure of $60 \mathrm{lbf}$ per sq. $\mathrm{ft}$.

D. Forced Entry Resistance: Windows shall comply with requirements for Grade 40 level of resistance to forced entry when tested in accordance with ASTM F588.

\subsection{SUBMITTALS}

A. Product Data: Submit product data for each type of wood window specified, including standard construction details, dimensions of individual components, profiles, finishes, hardware, and accessories.

B. Shop Drawings: Submit shop drawings for each type of window specified, including $1 / 4$-inch scale wall elevations, typical unit elevations at 3/4-inch scale, glazing details, and fill-size details of typical composite members.

\subsection{QUALITY ASSURANCE}

A. Wood Window Standard: Comply with provisions of NWWDA I.S. 2 for standards of performance and fabrication workmanship for wood windows.

B. Glazing Standards: Comply with recommendations of the Flat Glass Marketing Association (FGMA) "Glazing Manual" and "Sealant Manual" except where more stringent requirements are indicated. Refer to those publications for definitions of glass and glazing terms not otherwise defined in this section or referenced standards.

c. Safety Glass Standard: Where safety glass is indicated or required by authorities having jurisdiction, provide the type of products indicated which comply with ANSI Z97.1 and testing requirements of 16 CFR Part 1201 for category II materials. 
D. Insulating Glass Certification Program: Provide insulating glass units permanently marked either on spacers at least one component pane of units with the appropriate certification label of inspecting and testing organization indicated below.

A. Insulating Glass Certification Council (IGCC)

B. Associated Laboratories, Inc. (ALI)

E. Single Source Responsibility:Provide wood windows produced by a single fabricator who is capable of indicating prior successful production of units similar to those required.

\subsection{PROJECT/SITE CONDITIONS}

A. Field Measurements:

A. Check actual window openings in construction work by accurate field measurement before fabrication of custom window units. Show recorded measurements on final shop drawings.

B. Coordinate fabrication with construction progress to avoid delay. Where necessary, proceed with fabrication without measurements, and coordinate tolerances to ensure proper fit of window units.

\subsection{WARRANTY}

A. Submit a written warranty signed by the Manufacturer, agreeing to repair or replace wood window units that fail in materials or workmanship within the specified warranty period.

A. Failures include, but are not limited to, structural failures, including excessive deflection, excessive leakage, air infiltration, failure of weatherstripping, faulty operation of window sash or hardware, and deterioration of metals, finishes, and other materials beyond normal weathering.

B. Warranty period for wood windows is 3 years after the date of substantial completion.

C. This warranty shall be in addition to and not a limitation of other rights the Government may have against the Contractor under the Contract Documents.

PART 2---PRODUCTS

\subsection{MANUFACTURERS}

A. Pella Corp.

102 Main Street

Pella, IA 0219

515/628-1000

B. Andersen Corporation

100 Fourth Avenue North

Bayport, MN 55003

612/439-5150

\subsection{MATERIALS}

A. General: Comply with requirements of NWWDA I.S. 2.

B. Wood: Clear Ponderosa Pine or other suitable fine-grained lumber that has been kiln-dried to a moisture content of 6 to 12 percent at time of fabrication and is free of visible finger-joints, blue stain, knots, pitchpockets and surface checks larger than 1/8-inch deep by 2-inches wide.

1. Lumber shall be water-repellent preservative treated after machining in accordance with NWWDA I.S. 4.

C. Aluminum Cladding: Provide manufacturer's standard aluminum formed sheet or extruded cladding mechanically bonded to exterior wood sash and frame members.

1. Trim members: Aluminum clad wood trim.

2. Finish: Provide factory-applied baked-on enamel finish.

3. Color: Custom color as selected by the Architect from the manufacturer's standard color range.

D. Anchors, Clips, and Accessories: Fabricate anchors, clips and window accessories of aluminum, non-magnetic stainless steel, or hot-dip zinc coated steel complying with ASTM A123; provide strength sufficient to withstand design pressure indicated.

E. Fasteners: Comply with NWWDA I.S. 2 for fabrication and with manufacturer's recommendations and standard industry practices for type and size of installation fasteners.

A. Use zinc-coated or nonferrous nails and screws for window fabrication and installation.

B. Use brass screws for hardware and accessory installation. 
F. Hardware: Provide the manufacturer's standard hardware, necessary to properly operate, tightly close, and securely lock windows. Do not use aluminum in frictional contact with other metals.

A. Provide solid bronze hardware, with plated steel orbrass/bronze operating bars and rods.

G. Compression Weatherstripping: Provide compressible weatherstripping, designed for permanently resilient sealing under bumper or wiper action, completely concealed when sash is closed.

$\mathrm{H}$. Glass and Glazing Materials: Provide the manufacturer's standard clear sealed insulating safety glazing material that complies with ANSI Z97.1.

A. Insulating glass unit shall have metal spacers, sealed between the panes, behind each muntin bar.

I. Glazing Seal: Provide the manufacturer's standard extruded vinyl or butyl glazing gasket providing weathertight seal.

J. Sliding Weatherstripping: Provide woven pile weatherstripping of polypropylene, wool, or nylon pile, with resin-impregnated backing fabric, and aluminum backing strip; comply with AAMA 701.2.

\subsection{EQUIPMENT}

A. General: Comply with minimum operating requirements of NWWDA I.S. 2.

B. Casement Windows: Provide units containing side-hinged swing-out sash, with the following equipment and hardware:

1. Hook or cam-type latch and lever, for operation without screen removal.

2. For sash more than 48 inches high, provide 3 extension hinges and 2 -point latching mechanism.

C. Double-Hung Windows: Provide units containing 2 balanced, vertically-sliding sash with the following equipment and hardware. 2 pair concealed counterbalancing mechanism.

1. Latch at meeting rail.

2. Lift handle on the lower rail.

D. Hopper Windows: Provide units containing bottom-hinged (hopper) sash with the following equipment and hardware:

1. 2 balance support arms and pivots with friction shoes.

2. Cam latch or other latching hardware with lever handle or pull. Provide latch with eye for pole operation for operable sash located more than 6 feet above the floor.

\subsection{FABRICATION}

A. General: Except to the extent that more stringent requirements are indicated, provide the manufacturer's standard fabrication of units. Comply with indicated standards. Include a complete system for assembly of components and anchorage of window units.

1. Comply with requirements of referenced standards for moisture content of lumber at time of fabrication and for relative humidity conditions in the installation areas.

B. Fabricate windows to produce units that are re-glazable without dismantling sash framing. Provide openings and mortises precut, where possible, to receive hardware and other items.

C. Each window unit includes sash, frame, stops, sill (including undersill or nosing), and moldings, integral mullions and muntins, hardware, and accessories

D. Provide weatherstripping at perimeter of each operating sash.

1. Provide weatherstripping at perimeter of each operating sash.

a. For double/single-hung sash, provide weatherstripping only at horizontal rails of operable sash.

2. Provide glazing stops, nailed or snap-on, coordinated with glass selection and glazing system indicated

3. Preglazed Window Units: Preglaze window units at he shop before delivery.

E. Complete fabrication, assembly, finishing, hardware application, and other work before shipment to the project site, to the maximum extent possible. Disassemble components only as necessary for shipment and installation. Where necessary for fitting at site, provide ample allowance for scribing, trimming, and fitting.

F. Wood Finish: Provide the following finish on exposed wood in units:

1. 1Shop-Primed Units: Provide the fabricator's standard shop prime coat on exterior wood surfaces only.

\section{PART 3---EXECUTION}

\subsection{EXAMINATION}

A. Inspect openings before beginning installation. Verify that the rough or masonry opening is correct and the sill plate is level. Do not proceed with installation of window units until unsatisfactory conditions have beencorrected.

1. Masonry surfaces shall be visibly dry, and free of excess mortar, sand, and other construction debris. 
2. Wood frame walls shall be dry, clean, sound and well-nailed, free of voids, and without offsets at joints. Ensure that nail heads are driven flush with surfaces in the opening and within 3 inches of the opening.

\subsection{ERECTION, INSTALLATION, APPLICATION}

A. Comply with manufacturer's instructions and recommendations for installation of window units, hardware, operators, accessories, and other window components.

B. Set units plumb, level, true to line, without warp or rack of frames or sash. Provide proper support and anchor securely in place.

C. Set sill members in a bed of compound or with joint fillers or gaskets as indicated, to provide weathertight construction. Coordinate window installation with wall flashings and other built-in components.

\subsection{ADJUSTING/CLEANING}

A. Adjust operating sash and hardware to provide a tight fit at contact points and weatherstripping, and to provide smooth operation and a weathertight closure. Lubricate hardware and moving parts.

B. Clean interior and exterior surfaces promptly after installation of windows. Take care to avoid damage to protective coatings and finishes. Remove excess glazing and sealants, dirt, and other substances.

C. Clean glass of preglazed window units promptly after installation. Wash and polish glass on both faces not more than 4 days prior to date scheduled for final inspection. Comply with manufacturer's recommendations for final cleaning and maintenance.

D. Remove and replace glass that is broken, chipped, cracked, abraded or damaged in other ways during construction period, including natural causes, accidents, and vandalism.

\subsection{PROTECTION}

A. Institute and maintain protection and other precautions required through remainder of construction period to ensure that, except for normal weathering, window units

B. will be without damage or deterioration at the time of substantial completion. 


\section{Stage III - Doors}

Original wood doors are important features on historic buildings. Their spacing contributes to the visual rhythm of the facade, while providing insight into the interior spaces and uses of the building. Their design, craftsmanship, and materials contribute to a property's historic value. Historic solid and paneled wood doors have good thermal properties and should be retained.

Major signs of wood door deterioration include moisture, dry rot, insect damage, splitting, and peeling paint. The causes of deterioration and careful analysis of the door deterioration (supplemented by testing) is vital to the success of any historic wood repair project. Repair of a wood door may consist of either patching the historic material or filling in with new material to match the historic material. If replacement is necessary, duplication of historic materials and details should be as exact as possible to ensure a repair that is functionally and aesthetically acceptable.

\subsection{Immediate concern for wood doors}

Ensure that frames and doors have proper maintenance, regular painting or other finishing material, and that caulking and weatherstripping are applied, as necessary. Wood doors that are repaired and properly maintained will have an extended service life, while also contributing to the historic character of the building. Improper maintenance can cause long-term deterioration of wood elements. If deterioration occurs, selective repair or replacement of damaged parts and implementation of a regular maintenance program is often all that is required to retain a historic door.

All the following actions should be taken to avoid deterioration of wood doors:

- Verify that doors fit properly in their frames and joints are tight.

- Verify that thresholds are installed properly.

- Remove thresholds that are deteriorated beyond rehabilitation -and replace with in-kind materials to inhibit water and insect damage.

- Remove damaged or peeling paint to expose the next sound layer by using the gentlest methods possible; then repaint or otherwise refinish the wood element. 
- Repair damaged wood and treat as per preservation standards.

- Replace a severely damaged door, if needed, with an in-kind door; consult original plans for specifications.

- Inspect the wood to determine whether repainting is necessary or if cleaning is all that is required.

- Make repairs to wood after cleaning the surface gently, if necessary.

- Maintain undamaged wood doors and reapply paint or stain as needed to prevent the wood from deteriorating.

All of the pedestrian doors on Bldg. 550 are original from 1942. It is rare that over time the doors were not replaced. It is important to consider the size, shape, door frame, panes, the opening mechanisms, and the hardware when evaluating doors. There are four types of doors found on Bldg. 550 that were of standard design used throughout the construction of the WWII buildings at Camp McCoy in 1942 (see Figure 62). One type was labeled "Type D" on the original drawings, and are, according to the Standard Windows Details sheet, Plan 800-151, 6'-8" tall by 2'-8" wide and had a four-pane divided-light on the top half and three inset wood panels on the bottom half (see Figure 63). "Type C" doors were a set of two doors that measured 6'-8" tall and total of 5'-4" wide (see Figure 64).

There were originally three large overhead doors on the west elevation of Bldg. 550. According to original drawings Plan Number 800-801 these doors were a mixture of wood and glass (see Figure 65). These have since been removed and replaced with metal overhead doors. There was one standard type interior door used in Bldg. 550, "Type E," which measured 6', 8" tall by 2', 8" wide and was wood five-panel door (see Figure 66). Interior wooden screen doors were located at the single-entry doors. According to HABS documentation, the exterior of the wood doors would have been painted a medium gray color. The interior of the doors was typically painted and possibly in the same medium gray color. It is unknown if the interior screen doors were left unpainted or painted. 
Figure 62. Plan 800-154, Standard Door Details, 1942.

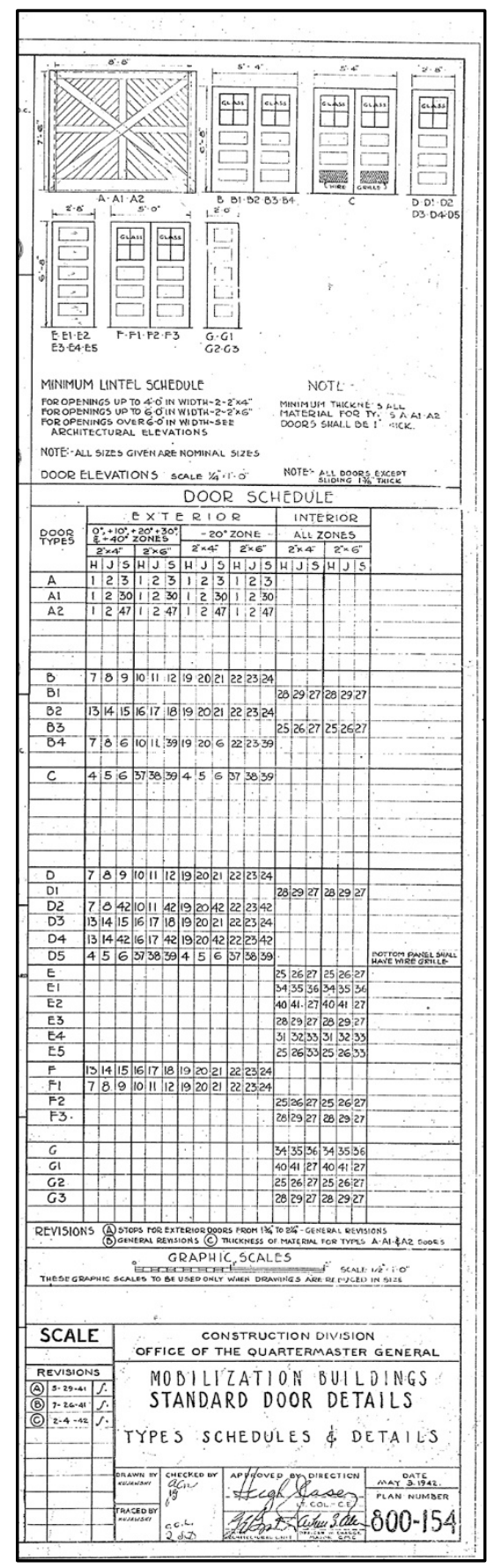

Source: Fort McCoy DPW. 
Figure 63. Close-up detail of standard door “Type D,” 1942.

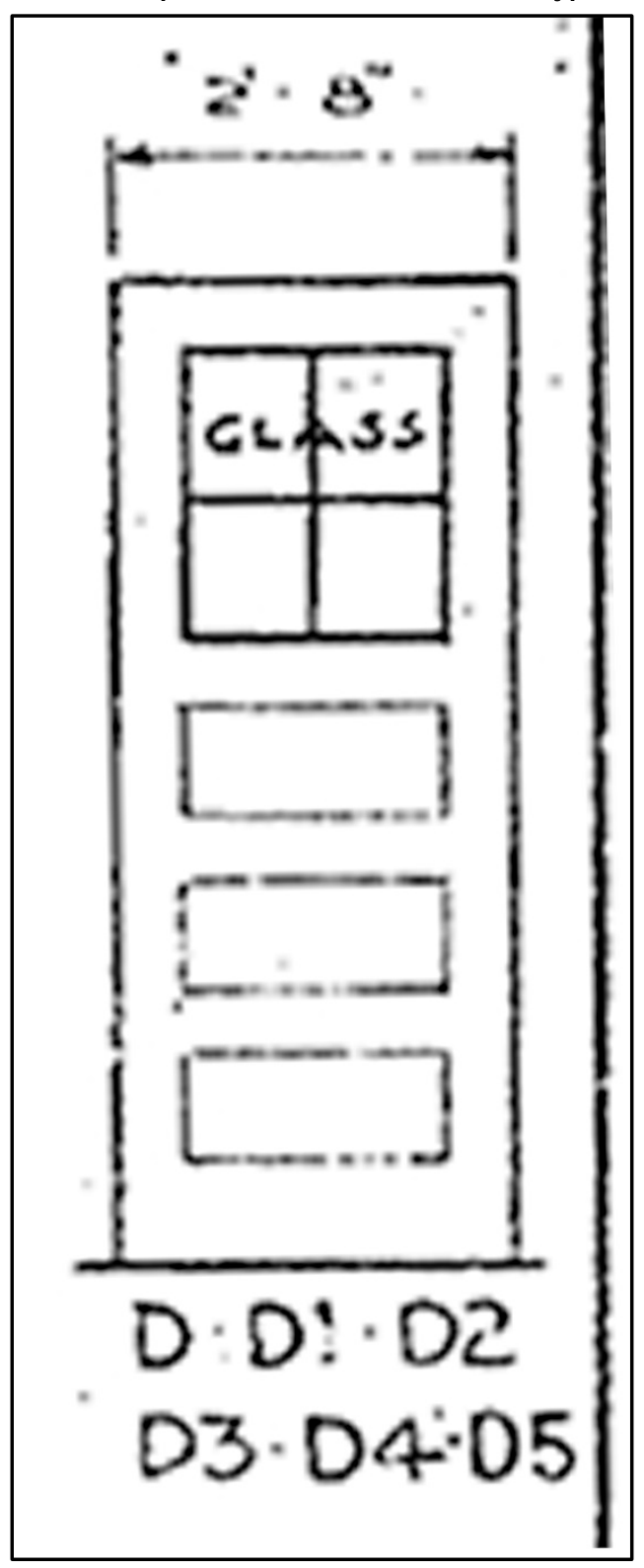

Source: Fort McCoy DPW. 
Figure 64. Close-up detail of standard door “Type C," 1942.

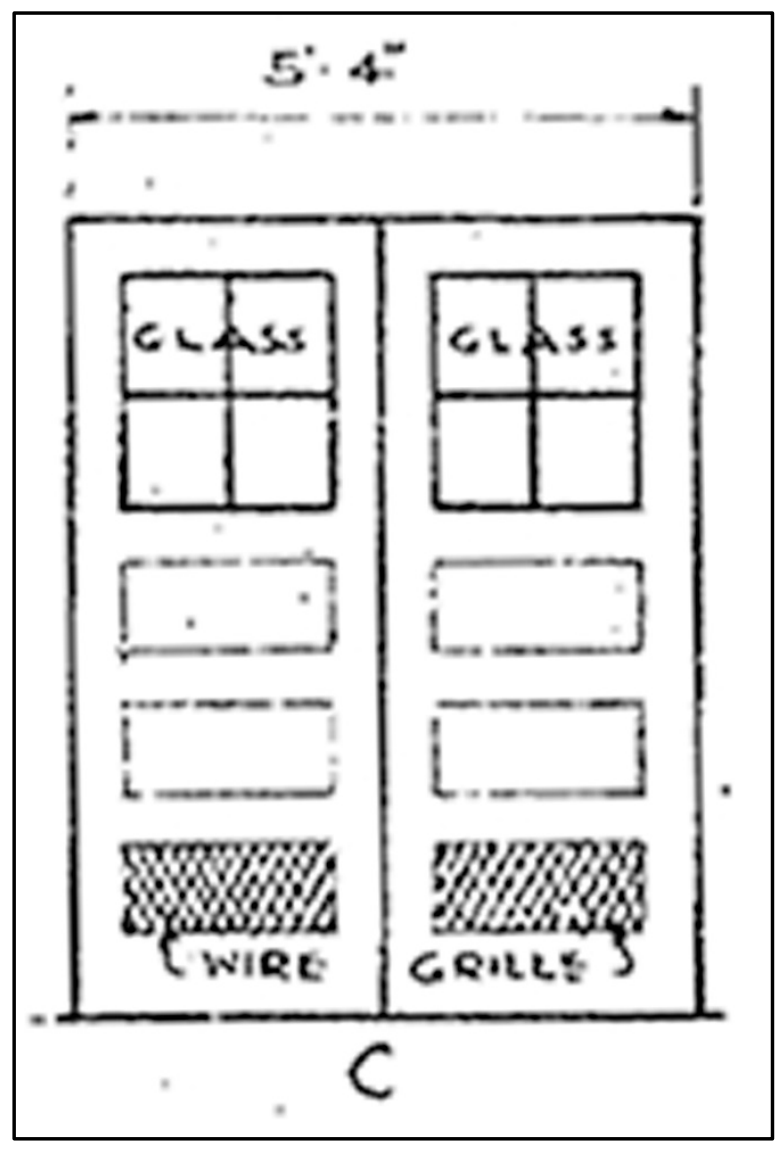

Source: Fort McCoy DPW. 
Figure 65. Detail of wood and glass overhead doors for Bldg. 550, Plan 800-801, 1942.

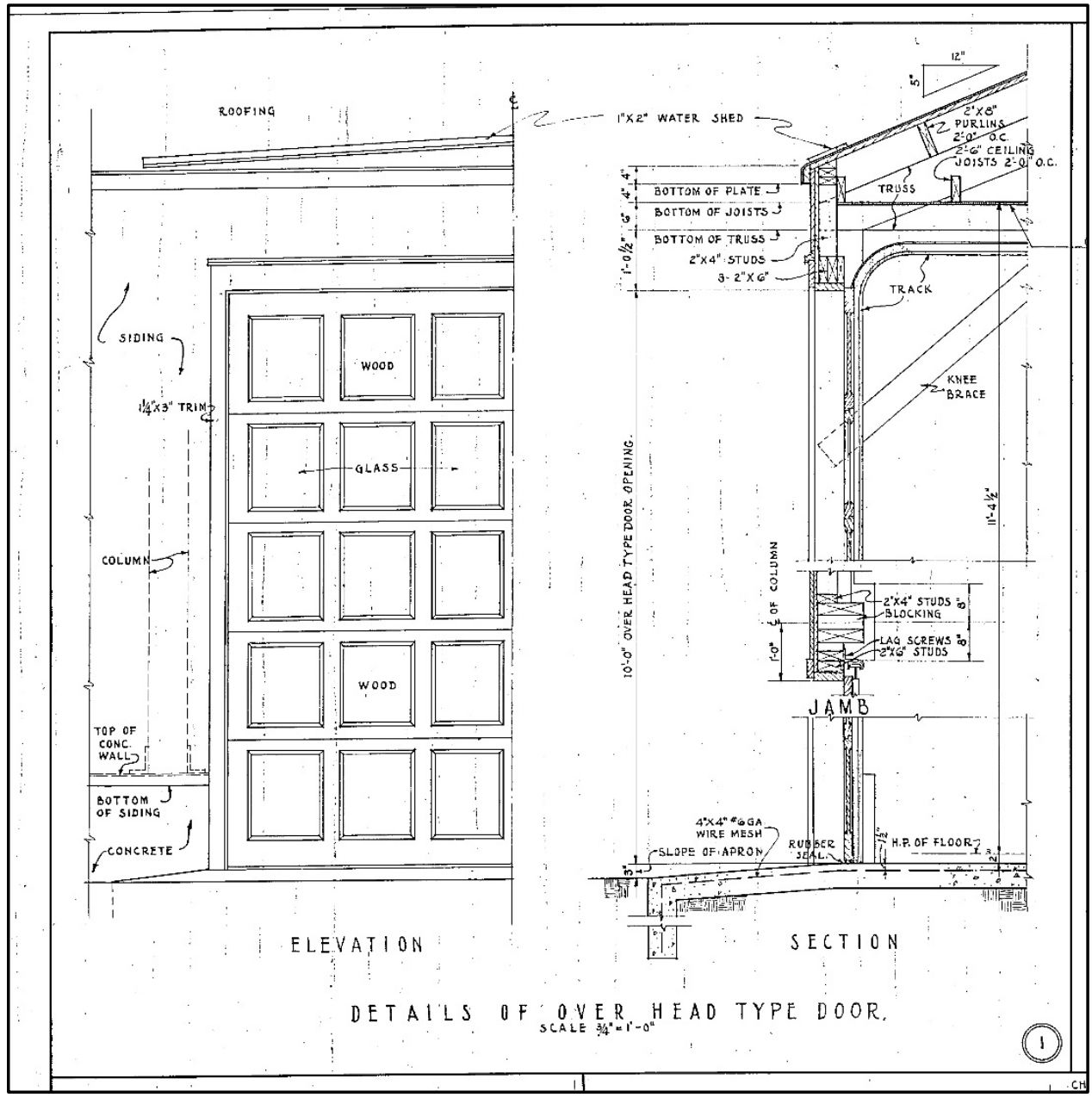

Source: Fort McCoy DPW. 
Figure 66. Close-up detail of standard door “Type E," 1942.

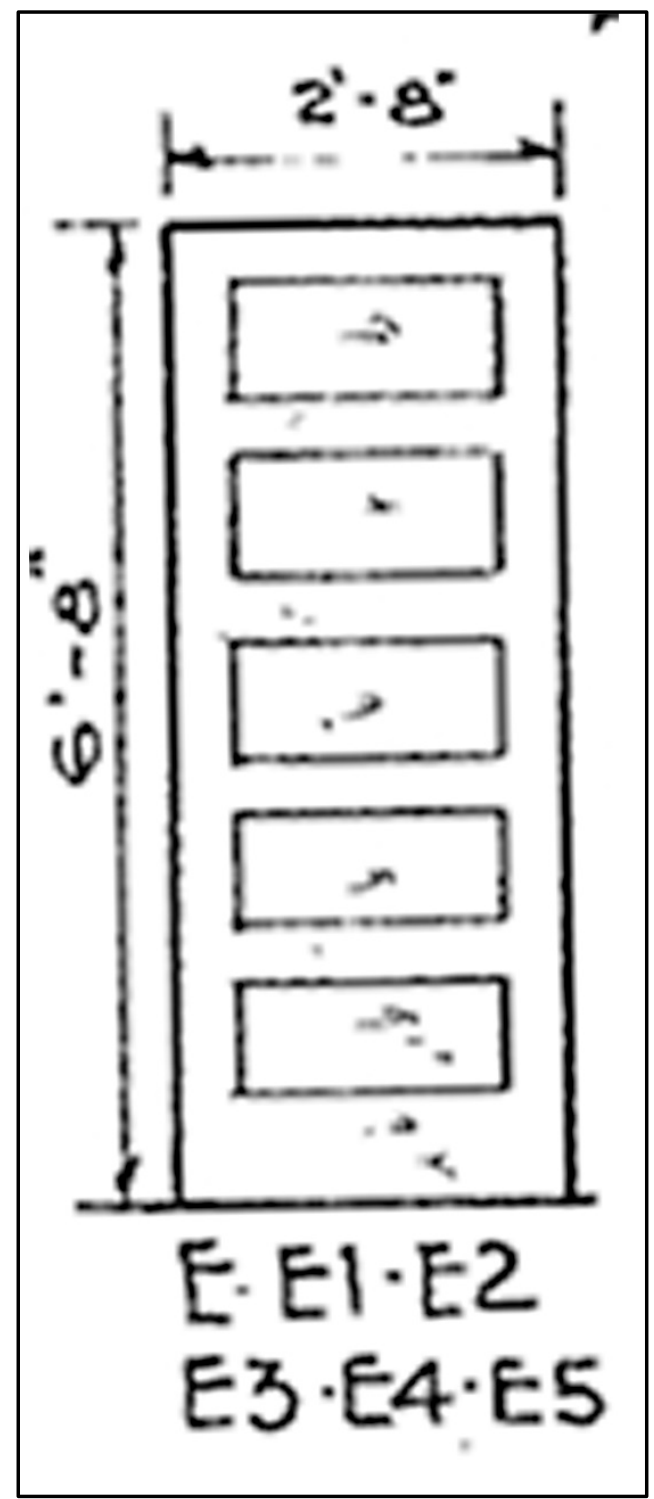

Source: Fort McCoy DPW.

\subsection{Exterior wood doors}

There are two single-entry doors, “Type D," which were on the north elevation into the apparatus room and on the east elevation into the squad room (see Figures 67 and 68). There was one "Type C" doors used on the north elevation and provided access into the heater room (see Figure 69). An original interior screen door is found at both single-entry "Type D" doors (see Figure 70).

The condition of the door on the north elevation are FAIR. There is some staining on the painted surfaces of the door and the trim (see Figure 67). The 
condition of the door on the east elevation is POOR. The majority of the paint has deteriorated, leaving the wood exposed and vulnerable to the elements (see Figure 68). The condition of the doors into the heater room on the north elevation are POOR. They appear to be slightly off hinges, which allows air, debris, and other elements into the building. The paint is peeling (see Figure 69). The hardware on all of the doors is POOR and showing signs of rust. The condition of the screen door is FAIR (see Figure 70). Screen doors can be maintained in the same manner as regular wood doors.

Figure 67. Original divided-light and wood panel “Type D" door on the north elevation into the apparatus room of Bldg. 550, 2018.

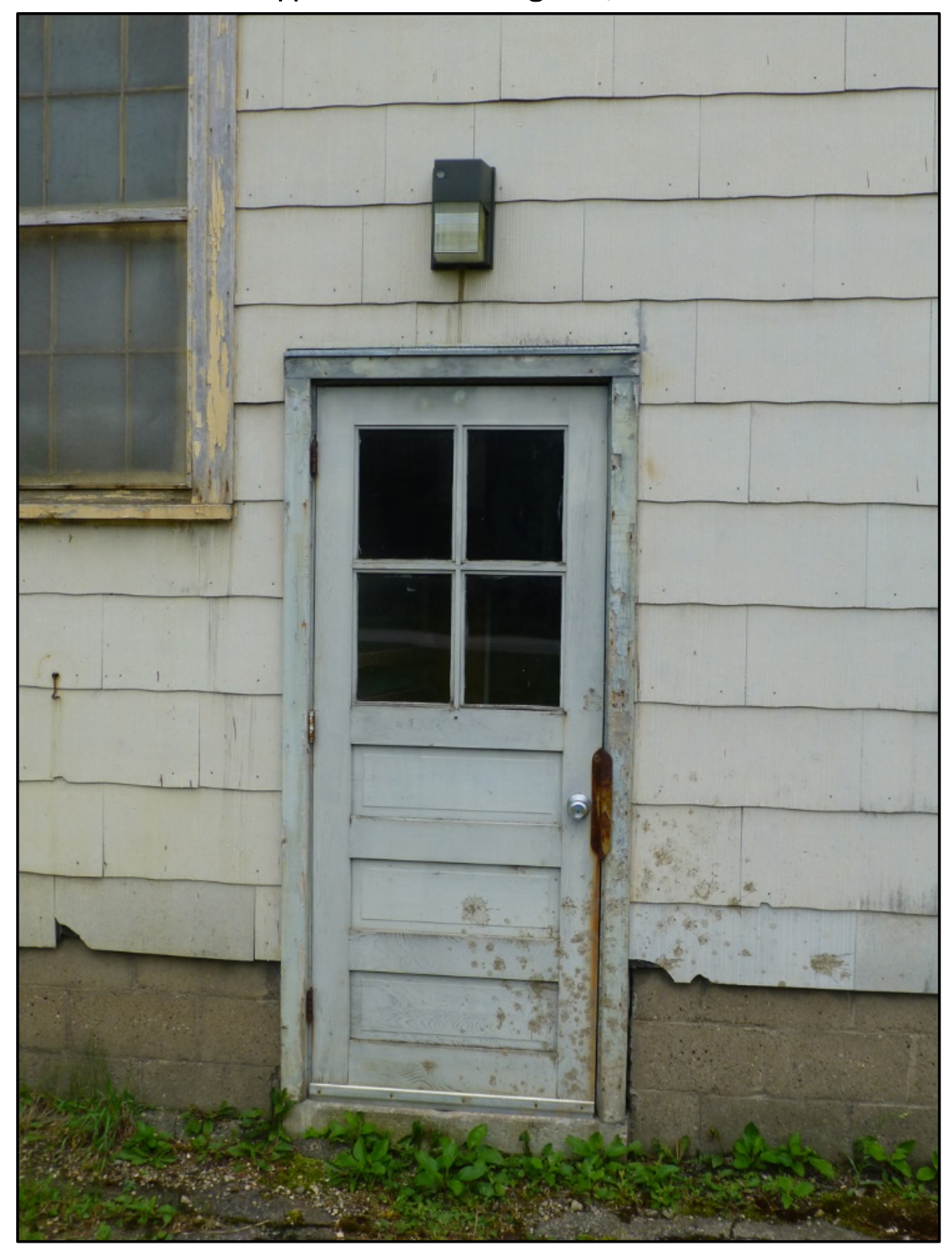


Figure 68. Original divided-light and wood panel “Type $D$ ” door on the east elevation leading into the squad room in Bldg. 550, 2018.

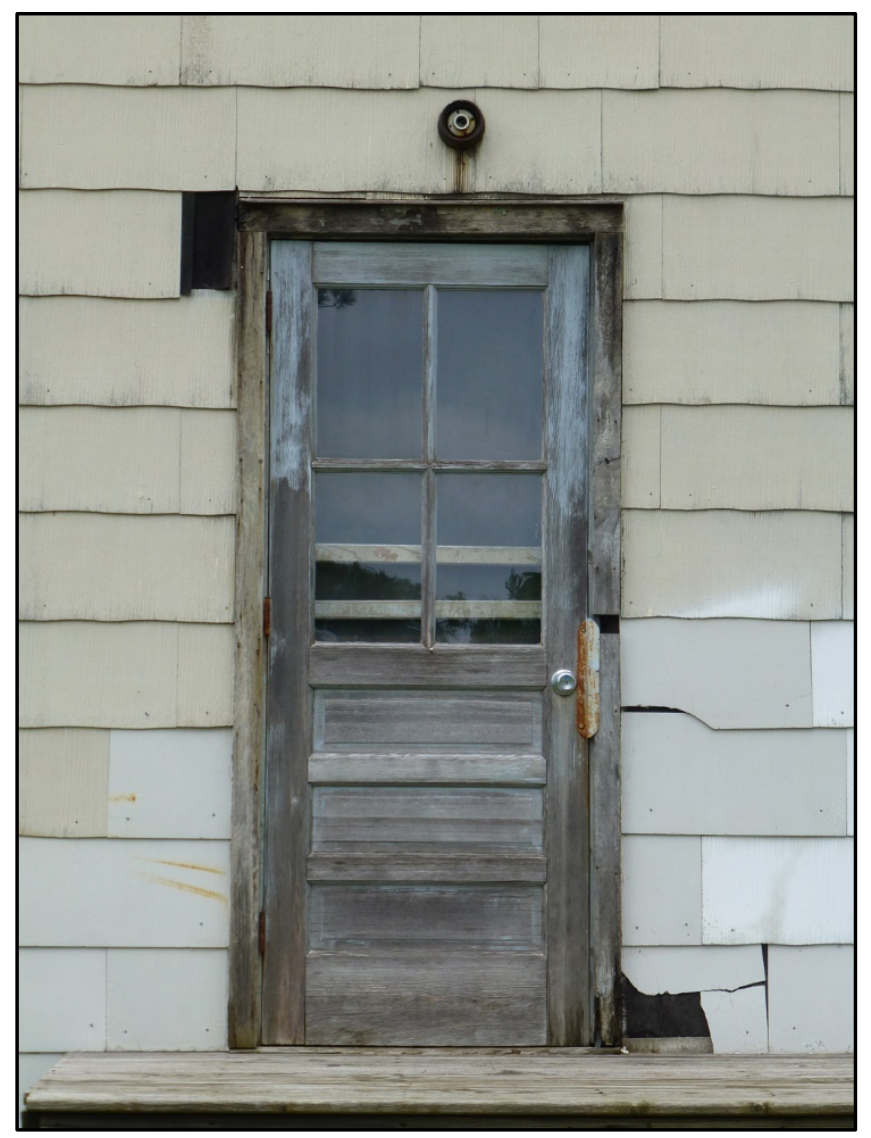

Figure 69. Original set of divided-light and wood panel doors "Type C" on the north elevation into the heater room in Bldg. 550, 2018.

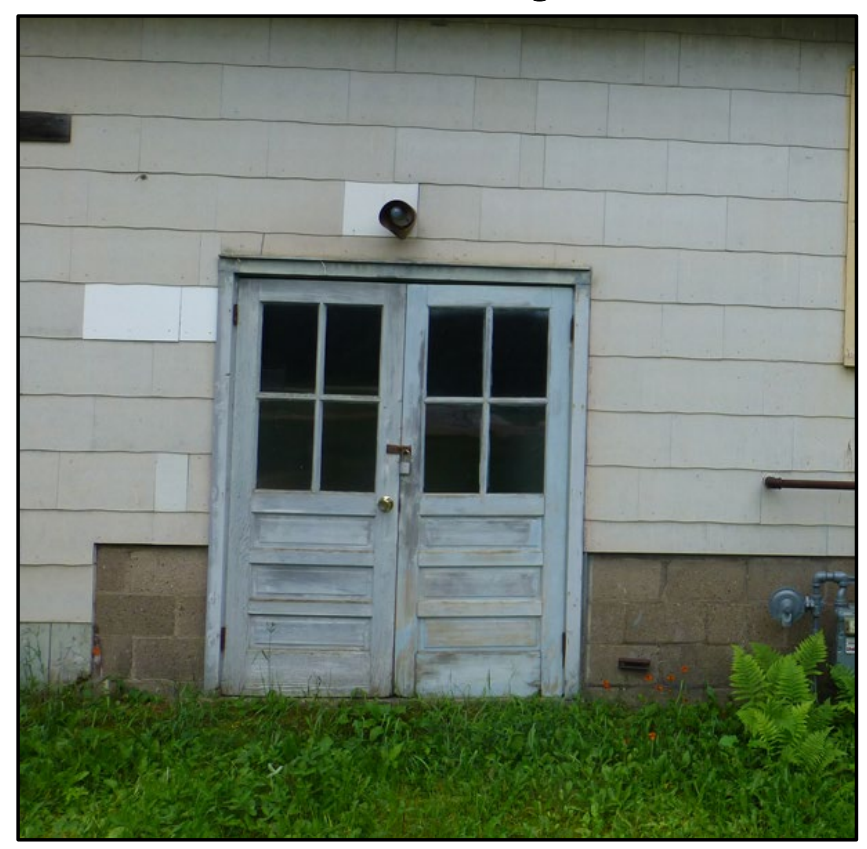


Figure 70. Looking at original divided-light and wood door "Type D" with original interior wooden screen door on the north side of Bldg. 550, 2018.

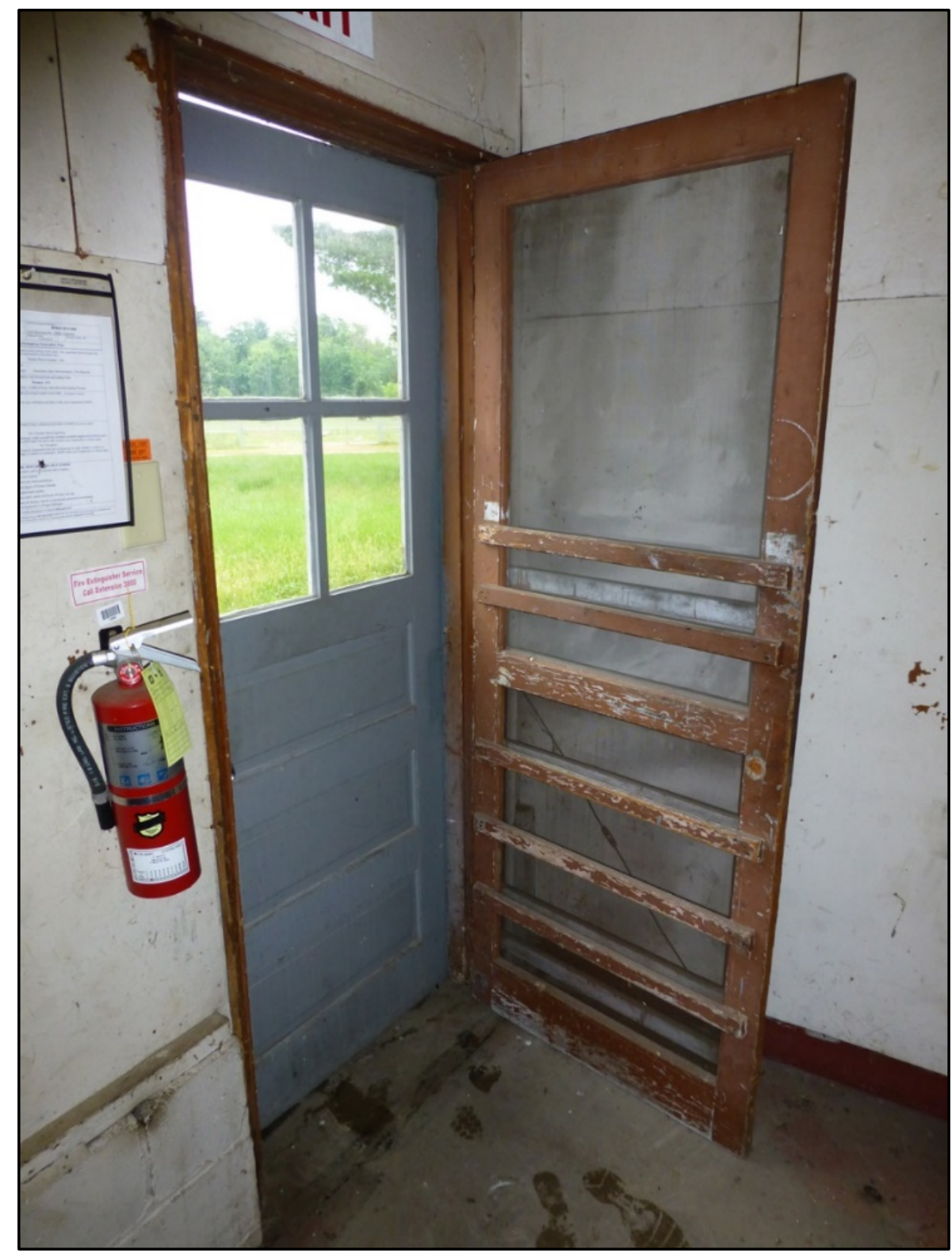

There are three replacement metal overhead doors on the west side of Bldg. 550. These are not in-kind to the original wood and glass doors (see Figure 71), and any renovation/rehabilitation plan of Bldg. 550 should include recreating these doors. 
Figure 71. Replacement metal overhead doors on the west elevation of Bldg. 550, 2018.

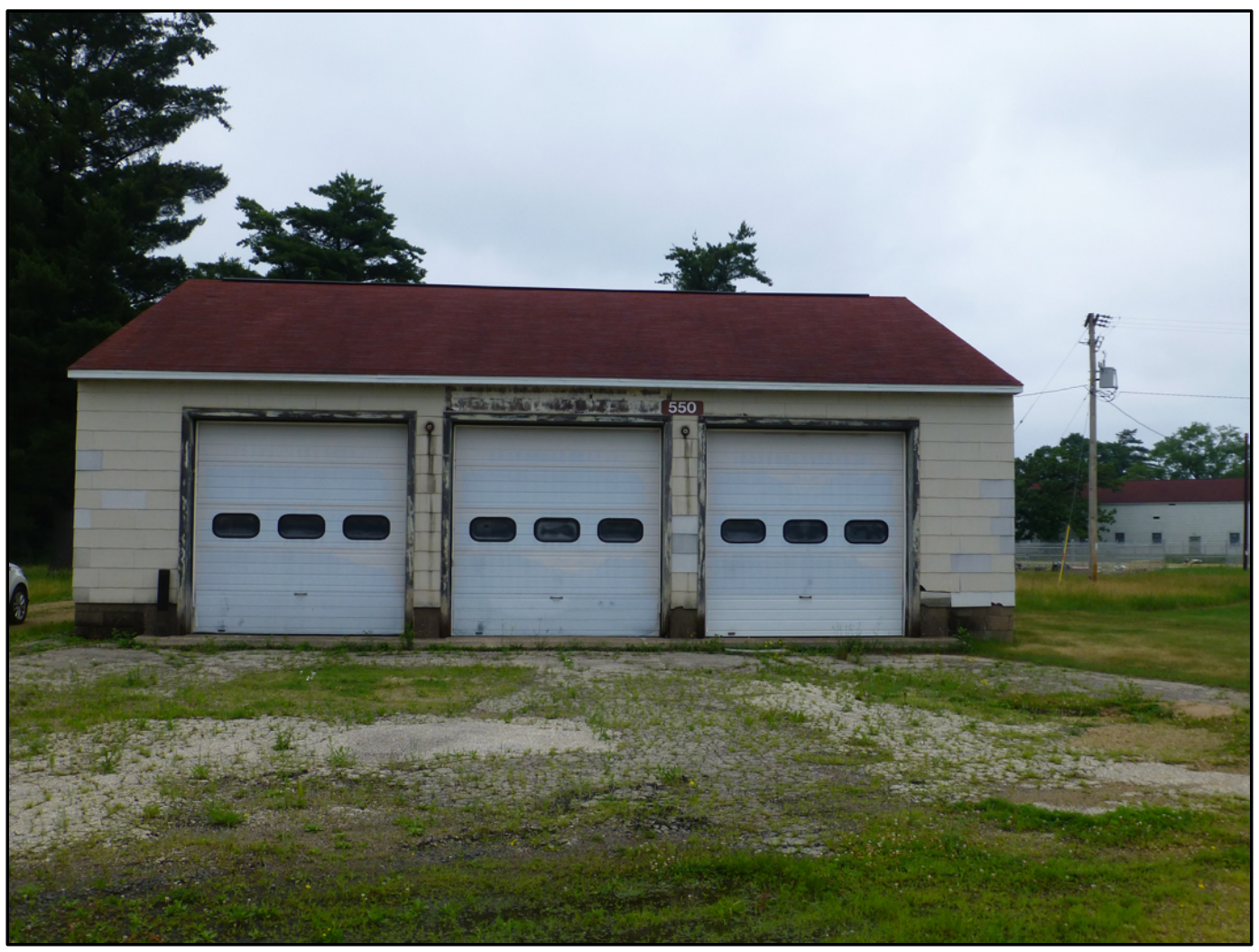

\subsection{Interior wood doors}

According to the original floor plan, there were six "Type E" wood panel interior doors. The doors are in FAIR condition (see Figure 72). The doors have been painted over time and some of the door trim has been removed. 
Figure 72. Looking at original wood panel interior door “Type E," 2018.

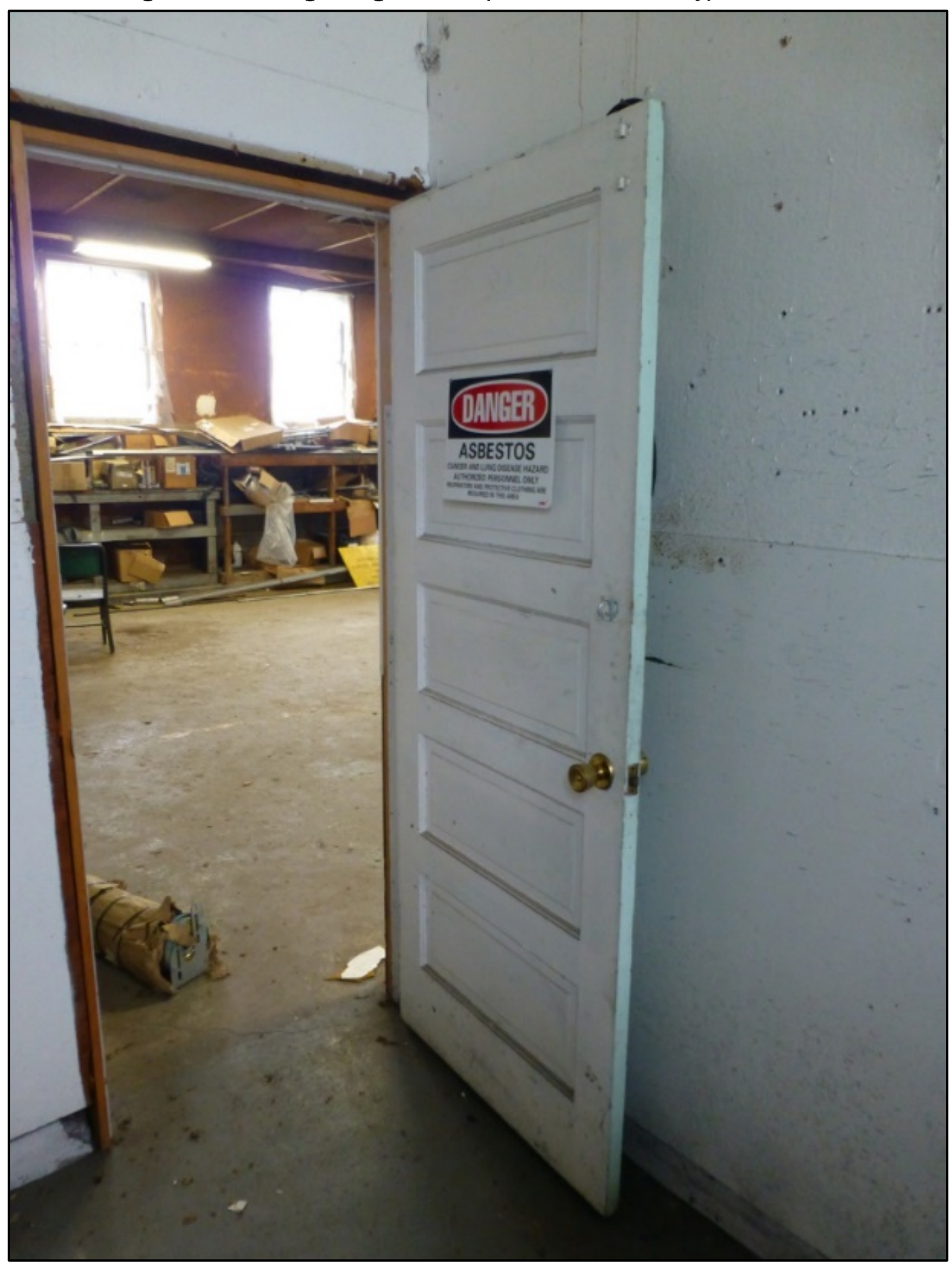

The 2018 photo shown in (Figure 73) appears to be a metal interior door. The plans indicate that this was simply a cased opening, and there are no metalcovered doors in the door schedule. The door appears to be a later addition. 
Figure 73. Looking at non-original door in Bldg. 550, 2018.

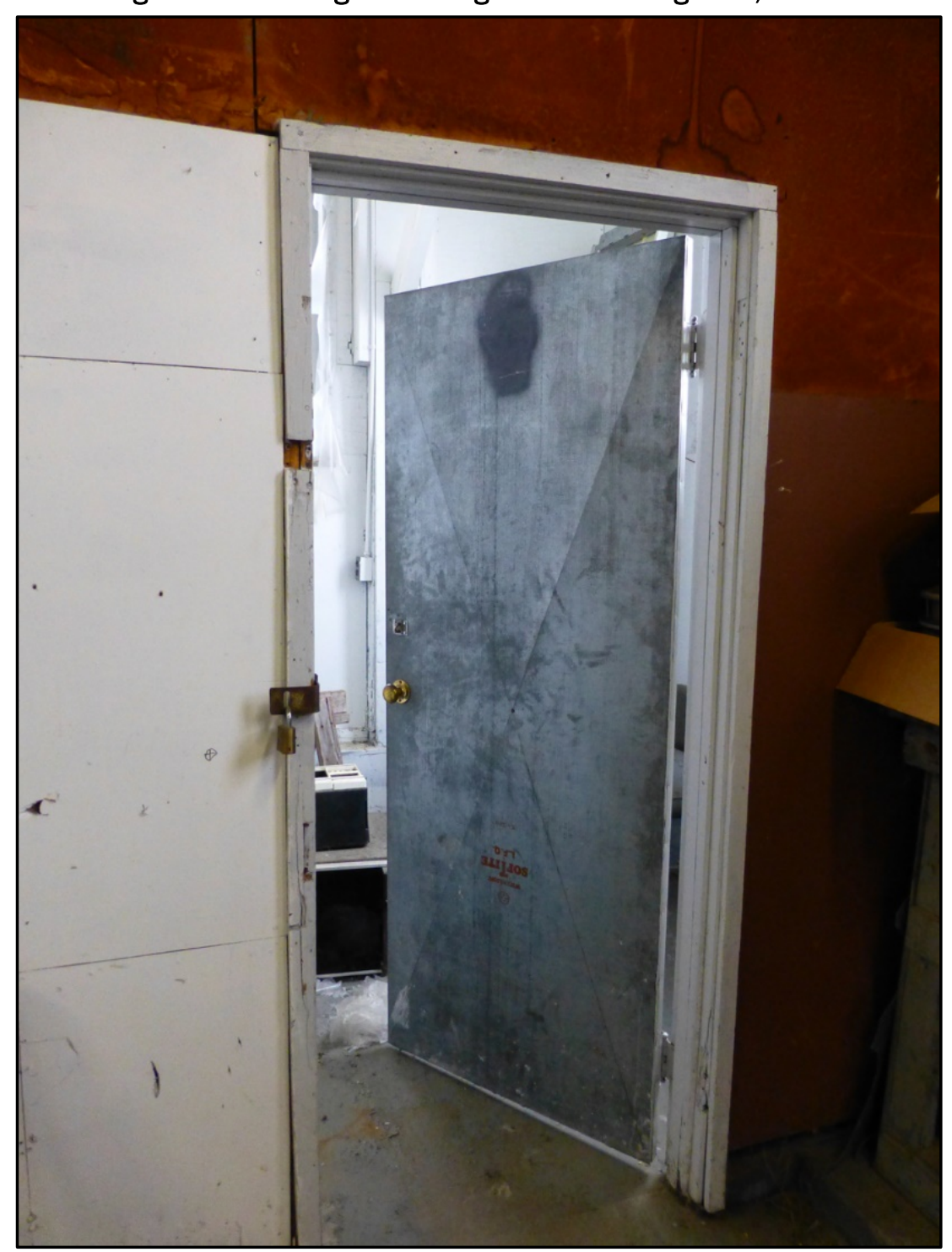

\subsection{Treatment measures}

Bldg. 550 has original wooden doors from their 1942 installment. Below are documents that inform how to refinish or repair wooden doors that have potential issues based on their age. 
6.4.1 Stripping and refinishing stained and varnished wood doors, ${ }^{17} 2017$ (GSA.gov).

\section{Stripping and Refinishing Stained and Varnished Wood Doors}

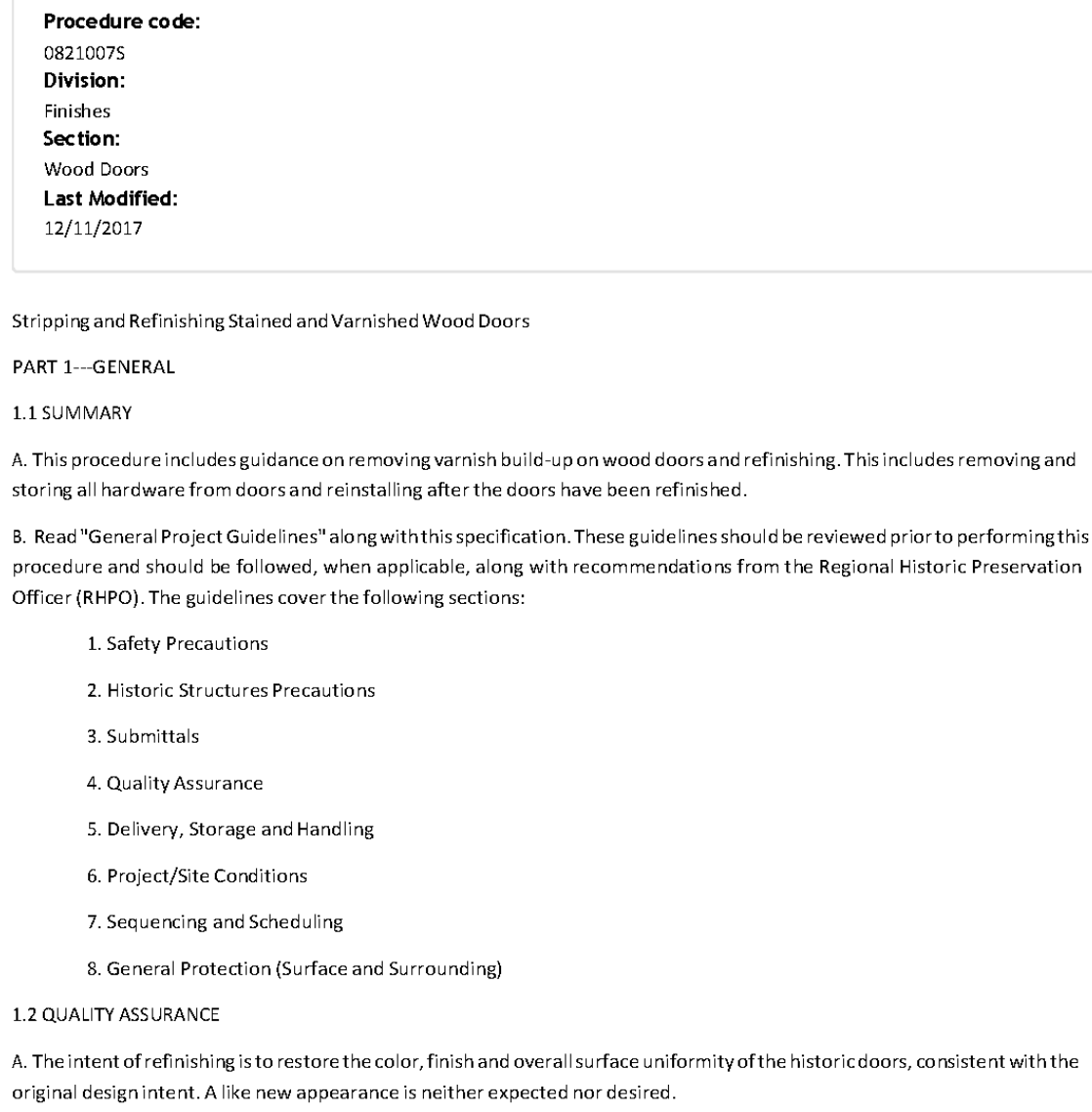

17 Retrieved from: https://www.gsa.gov/real-estate/historic-preservation/historic-preservation-policytools/preservation-tools-resources/technical-documents. 
B. Splotches, streaks, runs or other inconsistencies caused by improper application of finishing products will not be accepted.

C. Regulatory Requirements: Comply with municipal and Federal regulations governing the refinishing operations, chemical waste disposal and scaffolding.

1.3 SUBMITTALS

Samples:

A. Submit sample of wood stain and finish for approval of Contracting Officer.

B. Prepare a sample refinishing area for review of color and finish.

1.4 PROJECT/SITE CONDITIONS

Determine that surfaces to which finishes are to be applied are even, smooth, sound, clean, dry and free from defects affecting proper application. Correct or report defective surfaces to Contracting Officer.

1.5 CAUTIONS

A. Projects involving removal of coatings or use of hazardous chemicals are subject to employee safety and environmental laws governing lead paint disposal and use of volatile organic compounds (VOCs). Specified products may not be permitted or appropriate for all locations.

B. Products containing chemicals known to present health or environmental hazards should be used only as a last resort, where permissible, in accordance with manufacturer's directions and government requirements. Test milder formulations for effectiveness before proceeding to stronger alternatives.

C. Protect adjacent materials from damage or discoloration by cleaning run off. To avoid discoloring stone or driving stains deeper into porous stones, use the products and techniques described herein only for the combinations of dirt/stain and stone specified.

PART 2--PRODUCTS

2.1 MANUFACTURERS

A. Epifanes N.A., Inc.

B. Sherwin-Williams

C. Scotch-Brite (The 3MCompany)

D. W.M. Barr \& Co.

E. Samax Enterprises, Inc.

F. The Spic and Spancompany

2.2 MATERIALS

NOTE: Chemical products are sometimes sold under a common name. This usually means that the substance is not as pure as the same chemical sold under its chemical name. The grade of purity of common name substances, however, is usually adequate for stain removal work, and these products should be purchased when available, as they tend to be less expensive. Common names are indicated below by an asterisk $\left({ }^{*}\right)$.

A. Commercial Stripper suchas "KWIK Superfast Paint and Varnish Remover" (W. M. Barr \& Co.) "Rock Miracle" (Samax Enterprises, Inc.) or approved equal.

B. Stain: Penetrating, permanent oil-based stain such as "Wood Classics Interior Oil Stain", "Deckscapes Exterior Semi- 
Transparent Oil Stain" (Sherwin-Williams) or approved equal, colored to match existing interior and exterior wood.

C. Varnish: tung-oil modified phenolic spar varnish such as "Epifanes High Gloss Clear Varnish" (Epifanes N.A., Inc.) or approved equal.

D. Solvent: Mineral spirits, turpentine or denatured alcohol.

E. Mineral Spirits:

1. A petroleum distillate that is used especially as a paint or varnish thinner.

2. Other chemical or common names include Benzine* (not Benzene); Naphtha*; Petroleum spirits*; Solvent naphtha*. 3. Potential Hazards: TOXIC AND FLAMMABLE.

4. Safety Precautions:

a. AVOID REPEATED OR PROLONGED SKIN CONTACT.

b. ALWAYS wear rubber gloves when handling mineral spirits.

c. If any chemical is splashed onto the skin, wash immediately with soap and water.

5. Available from construction specialties distributor, hardware store, paint store, or printer's supply distributor. F. Turpentine:

1. Typically used as a solvent and thinner.

2. Potential Hazards: TOXIC AND FLAMMABLE.

3. Safety Precautions:

a. Work in a well-ventilated area.

b. Observe safety rules as turpentine is flammable, and the fumes can trip an ionization smoke detection system.

c. Store soiled cloths in a metal safety container to guard against spontaneous combustion.

d. Available from hardware store or paintstore.

G. Denatured Alcohol:

1. Other chemical or common names include Methylated spirit*.

2. Potential hazards: TOXIC ANDFLAMMABLE.

3. Available from hardware store, paint store or printer's supply distributor.

4. Denatured alcohol should be a satisfactory substitute for ethyl alcohol for stain removing purposes.

H. Alternative solvent: A mixture of $75 \%$ toluene, $24 \%$ acetone and $1 \%$ butyl acetate.

I. Toluene (C7H8):

1. A liquid, aromatic hydrocarbon that resembles benzene but is less volatile, flammable and toxic, and is produced commercially from light oils from coke- oven gas and coal tar and from petroleum, and is used as a solvent, in organic synthesis and an antiknock agent for gasoline.

2. Other chemical or common names include Toluol. 
3. Potential hazards: TOXIC AND FLAMMABLE.

4. Available from chemical supply house, hardware store, paint store or printer's supply distributor.

J. Acetone $(\mathrm{C} 3 \mathrm{H} 6 \mathrm{O})$ :

1. A volatile fragrant flammable liquid ketone used chiefly as a solvent and in organic synthesis.

2. Other chemical or common names include Dimethyl ketone; Propanone

3. Potential Hazards: VOLATILE AND FLAMMABLE SOLVENT

4. Available from chemical supply house or hardware store.

K. Detergent containing trisodium phosphate, such as "Spic and Span Multi-Surface and Floor Cleaner" (The Spic and Span Company), or approved equal.

L. Steel Wool: Grade 000 steelwool.

M. Aluminum oxide sandpaper -220 grit.

N. Silicon carbide paper - 400 grit.

o. Bronze wool.

P. Sandpaper -80 and 120 grit.

Q. Shellac burn-in sticks.

R. Tack rag.

S. Clean cotton cloths.

T. Soft, natural bristle brushes.

U. Mild soap.

V. Abrasive pad such as "Scotch-Brite" or approved equal.

PART 3--EXECUTION

3.1 PREPARATION

A. Protection:

1. Make sure work area is well-ventilated and wear protective clothing and rubber gloves.

2. Do not allow smoking in the work area.

3. Place a fire extinguisher for Class B fires at entrances for emergency use.

4. Change clothes as often as necessary to be effective in cleaning.

5. Daily, dispose of all used solutions, finishing products, solvent residue and soiled rags in sealed noncombustible containers to prevent a fire hazard.

6. Protect all surfaces adjacent to wood being refinished.

7. Maintain a healthy level of air circulation within the space being treated. Regularly employ and maintain exhaust fans or other air moving devices to the satisfaction of the Contracting officer's Representative.

8. Curtain off areas being treated from other trades and occupants to prevent fumes from reaching other parts of the 
building.

9. Wear appropriate safety devices such as respirators fitted with the correct cartridge, gloves, and other protective clothing.

B. Surface Preparation:

1. Remove all non-original door louvers, panels and transom panels taking care not to damage the remainder of the door, frame orpaneling.

2. Replace the non-original elements with new wood which matches the species of the original wood and which matches the detailing of the original millwork.

3. Hardware: Remove existing hardware, door numbers, and other applied elements, and store for reinstallation.

4. Make minor repairs to doors as required:

a. Fill holes exceeding 1 inch in diameter with matching Dutchmen. See "Dutchman Repair of Wood Floorboards" for guidance.

b. Fill smaller holes with patching compound tinted to match wood. See "Filling of Holes in Wood Veneer Paneling" for guidance.

3.2 ERECTION, INSTALLATION, APPLICATION

A. Strip the existing varnish finish:

1. Wet steel wool with solvent and rub over the doors to remove varnish build-up and smooth out checked surface. 2. Replace soiled steel wool frequently with clean and continue with wiping process until a smooth, even-colored surface is achieved.

a. Use no water on wood surface under any circumstances.

b. Work only one 4' square area at a time. Work area should be within a comfortable arms reach.

c. If solvent affects the stained color of the wood, discontinue use and use an alternative solvent mixture as listed in Section 2.02Materials.

3. Allow surface to dry thoroughly; no less than 24 hours.

-OR-

1. Apply commercial stripper following manufacturer's instructions.

2. Wash the surface with acetone to remove stripper residue.

3. Lightly sand the surface with 220 grit al uminum oxide sandpaper as needed to remove carbon soiling and finish damage not removed by solvent application.

4. Wipe surface with a tack rag to remove traces of bronze wool, sand and dust prior to applying new finishes. B. Remove shallow scratches:

1. Lightly sand, in the direction of the grain only, to remove shallow scratches, against the grain sanding, and finish damage not removed by stripper application.

2. Remove scratches using 80 grit sandpaper.

3. Finish using 120 grit sandpaper until smooth surface is attained. 
4. Smooth surface sufficiently to ensure uniform stain absorption.

5. Wipe surface with a tack rag to remove traces of steel wool, sand, and dust prior to applying new finishes. C. Apply the stain:

1. Color mix stain to match original finish.

2. Apply stain to bare wood surfaces using a soft cloth or bristle brush.

3. Allow stain to set as required for proper color match and maximum surface uniformity.

4. Wipe off excess stain by rubbing parallel to the grain with a soft dry cloth.

5. Allow surface to dry for at least 24 hours.

D. Fill deep scratches and gouges with shellac burn-in sticks tinted to match the wood stain.

E. Apply the finish coating:

1. Makesure that surface is clean, level and free of defects. Promptly report to Contractor Officer's Representative any unanticipated conditions which may affect the quality of the finish.

2. Apply 3 coats of varnish using a brush or sprayer to produce a uniform sheen and appearance. 3 . Allow each coat to dry for at least 4 hours.

4. Lightly sand with $\# 400$ grit silicon carbide paper or rub with fine steel wool between coats.

5. Vacuum surface and wipe with a dry tack rag to remove all grit and dust prior to applying next finish coat.

6. After curing, lightly rub surface with fine steel wool to replicate original finish.

F. Clean hardware:

1. General:

a. For bronze and stainless steel hardware (door knobs, escutcheon plates, hinges and closers), clean using a mild soap and water.

b. For stubborn dirt and hard to clean areas, apply detergent with a Scotch-Brite abrasive pad or bristle brush. Rinse thoroughly and buff dry with soft cotton.

c. Remove grease on closers and hinges with sponge and detergent.

d. Scrape gently with a non-metallic spatula to remove paint drips.

e. See also "Cleaning and Polishing Brass-Plate" and "Cleaning and Polishing Solid Brass" for guidance.

2. If required, carefully remove adhesive residue, paint and varnish drips from escutcheon plates using a paint stripper.

a. Apply with softcloths.

b. If necessary, apply light pressure using a natural bristle brush.

3. If necessary, remove adhesive residue from door knobs using a mild solvent.

a. Test an inconspicuous area to avoid damaging the finish.

b. DONOT APPLY SOLVENTS WHICH MAY REMOVE PATINA.

G. Repair locksets: 
1. Repair inoperable locksets, reusing original knobs and escutcheons.

2. Where locksets are missing or irreparably damaged, furnish new locksets matching originals. Replicate existing escutcheons in color, sheen, overall configuration, and detailing.

a. Conceal existing cutouts, but do not cover portions of door not originally concealed.

b. Replicate original knob's finish.

H. Install new closers where missing or irreparably damaged. Furnish closers matching originals in form and finish as closely as possible.

I. Replace glazing as required.

J. Clean glazing as required:

1. Remove adhesive residues, paint spatters, and other soiling using soft cloths and detergent.

2. Use a mild solvent and Scotch Brite pad or bristle brush to remove stubborn residues.

3. Remove paint splatters with solvent or by scraping gently with a razor blade held at a shallow angle.

4. DO NOT USE TOOLS OR CLEANING PRODUCTS WHICH MAY ETCH THE GLASS.

K. Reinstall kickplates and other hardware as required.

L. Remove and repaint louvers to match original as required.

M. Refinish jambs and frames to match original as required.

3.3 ADJUSTING/CLEANING

A. Adjust door to assure proper operation. Replace or rehang doors which are hinge bound and do not swing or operate freely. Replace worn hinge pins with replicates.

B. Refinish or replace job-finished doors damaged during installation.

C. For guidance on the periodic cleaning of woodwork see "Biennial Cleaning andStain Removal of Woodwork".

END OFSECTION 


\title{
6.4.2 Replacement of damaged wood doors, ${ }^{18} 2017$ (GSA.gov).
}

\section{Replacement of Damaged Wood Doors}

\author{
Procedure code: \\ 8210035 \\ Source: \\ National Capitol Region Specifications - Fed Trade Commission \\ Division: \\ Doors and Windows \\ Section: \\ Wood Doors \\ Last Modified: \\ 05/02/2017 \\ REPLACEMENT OF DAMAGED WOOD DOORS \\ PART 1---GENERAL \\ 1.01 SUMMARY \\ A. This procedure includes guidance on the removal and \\ replacement of deteriorated or damaged wood doors with \\ new to match original historic doors. It also includes \\ the removal, storage and reinstallation of original door \\ hardware. \\ B. See 01100-07-S for general project guidelines to be \\ reviewed along with this procedure. These guidelines \\ cover the following sections:
}

18 Retrieved from: https://www.gsa.gov/real-estate/historic-preservation/historic-preservation-policytools/preservation-tools-resources/technical-documents. 
1. Safety Precautions

2. Historic Structures Precaution $=\mathrm{s}$

3. Submittals

4. Quality Assurance

5. Delivery, Storage and Handling=

6. Project/Site Conditions

7. Sequencing and Scheduling

8. General Protection (Surface and Surrounding)

These guidelines should be reviewed prior to performing this procedure and should be followed, when applicable, along with recommendations from the Regional Historic Preservation Officer (RHPO).

\subsection{REFERENCES}

A. Architectural Woodwork Institute (AWI) www.awinet.org

1.03 System Description

A.

1. Architectural Woodwork Standards http://www.awinet.org/

2. Take all required field measurements and verify installation conditions for work.

\subsection{SUBMITTALS}

A. Shop Drawings: 
1. Indicate general construction, jointing methods, hardware locations and cut-outs for glazing and vents.

2. Indicate opening identifying symbol (room number), sizes, door type and grade, and show elevation, swing, light and grill cut-out sizes, locations and undercuts.

B. Samples:

1. Submit sectional sample of door construction indicating grade and species.

2. Submit sample of stain and finish for approval of Contracting Officer.

1.05 PROJECT/SITE CONDITIONS:

A. Field Measurements: Take all required field measurements and verify installation conditions for work of this section.

PART 2---PRODUCTS

2.01 MATERIALS

A. Wood doors, premium grade, to match existing species face and edge grain.

202 FABRICATION

A. Moisture content shall be $12 \%$ maximum at time of fabrication for all wood material. 
B. Fabricate doors in accordance with requirements of AWI

Quality Standards to match existing in construction and quality.

C. Allowable Tolerances for Fabrications of Doors:

1. Size: Plus or minus $1 / 16 "$ overall dimensions.

2. Maximum warp: 1/4"

3. Squareness: Length of diagonal measured on face of door from upper right corner to lower left corner between length of diagonal measured on upper left corner to lower right corner - maximum difference of $1 / 4^{\prime \prime}$.

4. Factory finish thickness: Minimum 1 mil., cured.

5. Show-through (photographing): 1/100" deviation from true plane in any $3 "$ span on door face.

D. Provide doors with minimum 1/2" thick edge strips (Door Stops); often found at 1 inch to $11 / 2$ inch wide and $3 / 8$ inch thick.

E. Make cut-outs and provide stops for glass in original locations using profiles matching the original.

F. Bevel strike edge of single acting doors $1 / 8^{\prime \prime}(3 \mathrm{~mm})$ in $2^{\prime \prime}(51 \mathrm{~mm})$.

G. Strike doors to receive hardware. Hardware locations to match its location on original doors. 
PART 3---EXECUTION

3.01 PREPARATION

A. Protection: Remove all non-original door louvers, panels and transom panels taking care not to damage the remainder of the door, frame or paneling.

B. Replace the non-original elements with new wood which matches the species of the original wood and which matches the detailing of the original millwork.

3.02 ERECTION, INSTALLATION, APPLICATION

A. Carefully remove existing damaged and altered doors to be replaced and label with location.

B. Remove all hardware from doors and store. New noncompatible hardware should be discarded and replaced with hardware matching the original.

C. Remove all glazing and vents and restore to original condition.

D. Fitting and Machining:

1. Fit doors for width by planning; for height by sawing.

a. $3 / 8$ inch maximum from bottom.

b. 1/8 inch maximum from top.

c. Bevel lock and hinge edges, $1 / 8$ " to $2 "$. 
2. Machine doors for Hardware.

3. Cut doors for glazing and vents. Cuts in replacement doors shall match size and location of openings cut in original doors.

4. Refinish all job site cut surfaces before final hanging of doors. See 08210-07-R for guidance on refinishing wood doors.

E. Install doors in accordance with requirements of AWI Standard and as indicated.

\section{Clearances:}

a. Allow maximum of $1 / 8^{\prime \prime}$ at jamb and head for fit doors.

b. Allow maximum of 3/16" over threshold or saddle.

c. Allow maximum of $3 / 8$ " over decorative floor coverings.

2. Install, using original hardware in original locations. Replace any damaged, incompatible, or missing hardware with that which is as close as possible to original.

3. Install original glazing and vents in original locations.

3.03 ADJUSTING/CLEANING

A. Replace or rehang doors which are hinge bound and do not 
swing or operate freely.

B. Refinish or replace job-finished doors damaged during installation. 


\section{Stage III - Concrete}

Major signs of concrete deterioration include cracking, spalling, deflection, stains, erosion, and corrosion. Whatever the causes of deterioration, careful analysis, supplemented by testing is vital to the success of any historic concrete repair project. Repair of historic concrete may consist of either patching the historic material or filling in with new material worked to match the historic material. If replacement is necessary, duplication of historic materials and detailing should be as exact as possible to ensure a repair that is functionally and aesthetically acceptable.

\subsection{Immediate concerns for concrete}

Deterioration of concrete can be caused by environmental factors, inferior materials, poor workmanship, inherent structural design defects, and inadequate maintenance. Environmental factors are a principal source of concrete deterioration. Concrete absorbs moisture readily, and this is particularly troublesome in regions of recurrent freeze-thaw cycles. Freezing water produces expansive pressure in the cement paste or in nondurable aggregates. ${ }^{19}$

Improper maintenance of historic buildings can cause long-term deterioration of concrete. Water is a principal source of damage to historic concrete, and prolonged exposure to it can cause serious problems. Unrepaired roof and plumbing leaks, leaks through exterior cladding, and unchecked absorption of water from damp earth are potential sources of building damage. ${ }^{20}$

Bldg. 550 has concrete features that are original to its 1942 design and construction. The west end of the building where the former apparatus room is located has a concrete block foundation with a concrete floor, while the rest of the building (living area/squad room) is supported by a concrete pier foundation system. Over time, concrete can spall, it can crack, and it can also shift.

\footnotetext{
19 Excerpt from Paul Gaudette and Deborah Slaton, Preservation Brief 15: Preservation of Historic Concrete: Problems and General Approaches. (Washington, DC: US Department of the Interior, National Park Service, Technical Preservation Services, n.d.) Available at: http://www.nps.gov/tps/how-to-preserve/briefs/15-concrete.htm. 20 ibid.
} 


\subsection{Concrete floors}

Concrete floors are in the apparatus room, the heater room, and the toilet room in Bldg. 550 (see Figures 74 and 75). The floor in the apparatus room is in FAIR condition, and it is the same with the floor in the toilet room. The researchers did not have access to the heater room. The floor in the apparatus room does have some major cracking, most likely due to the settling of structural support beam in the corner (see Figure 76).

Figure 74. Concrete floor in the apparatus room in Bldg. 550, 2018.

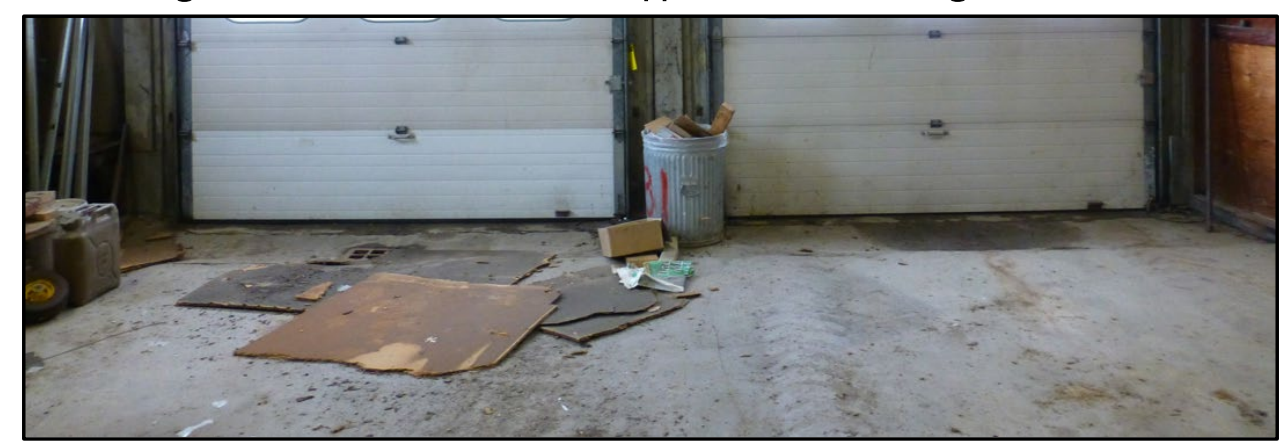

Figure 75. Concrete floor in the toilet room of Bldg. 550, 2018.

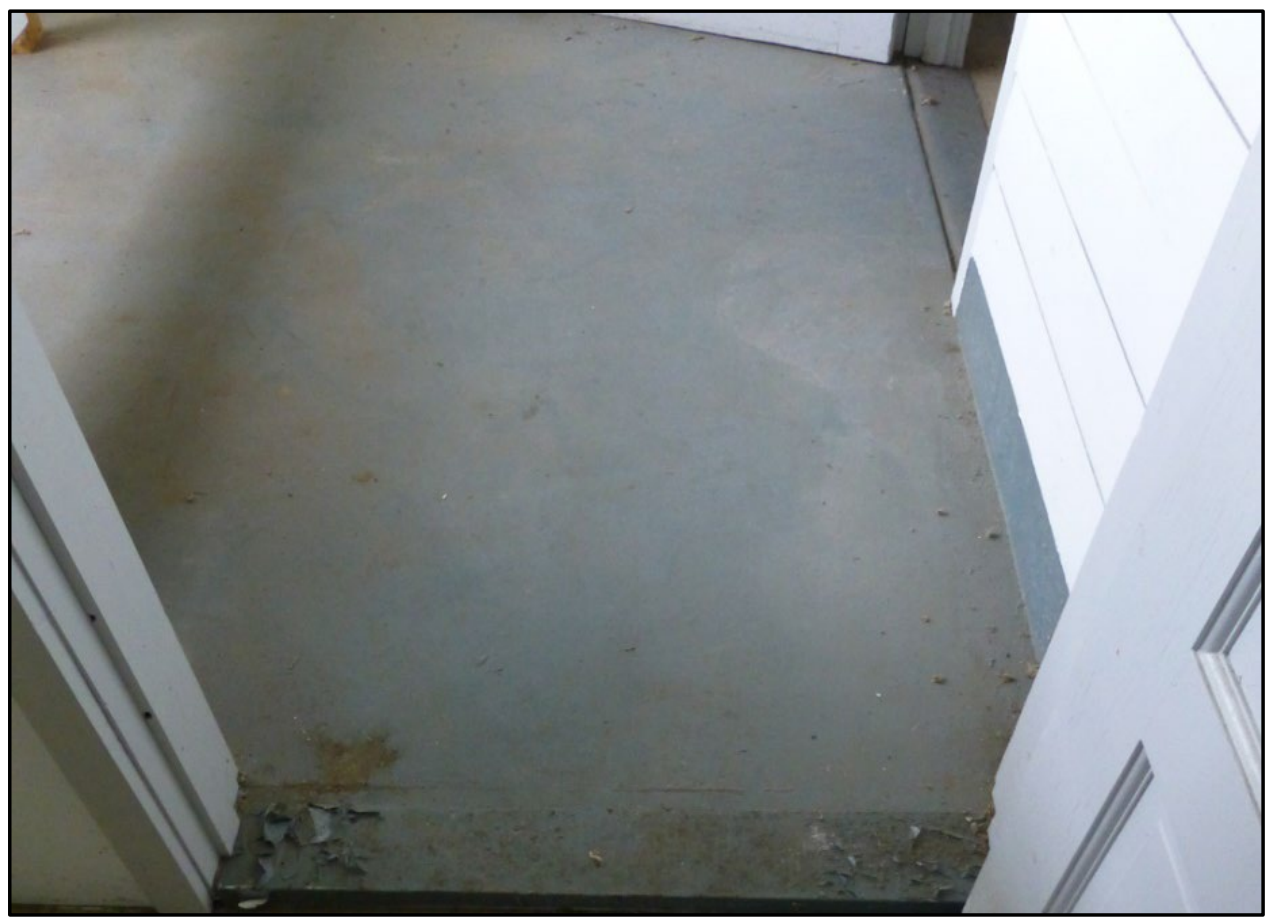


Figure 76. Looking at poured concrete floor in poor condition, 2018.

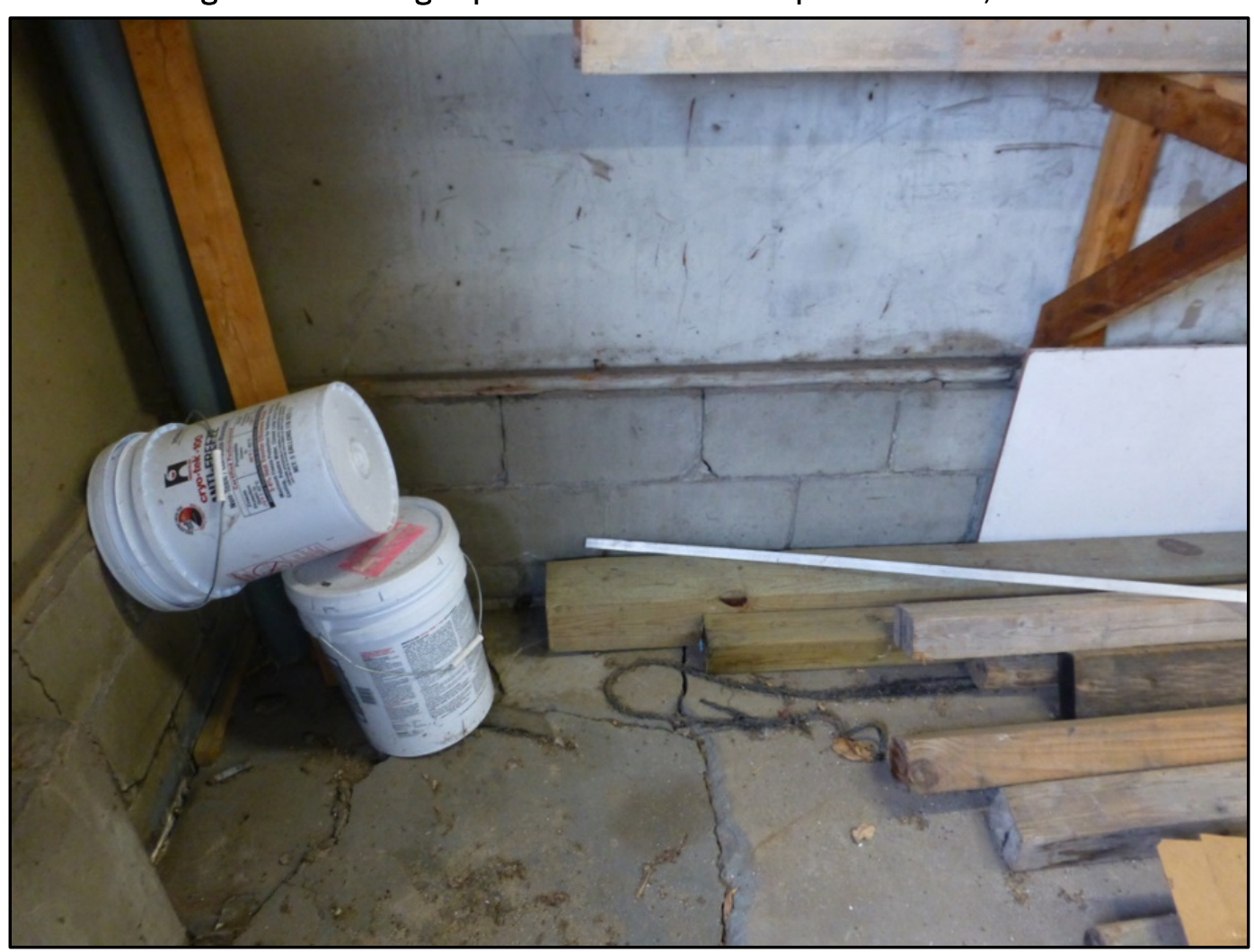

\subsection{Concrete block}

Concrete block walls were used in the apparatus room and the heater room of Bldg. 550 (see Figures 77 and 78). The condition of the concrete block is FAIR. In some areas there is major discoloration, and there appears to be patching. It is important to maintain concrete block of this manner because it is foundational. 
Figure 77. Concrete block is used for the heater room and the apparatus room in Bldg. 550, 2018.

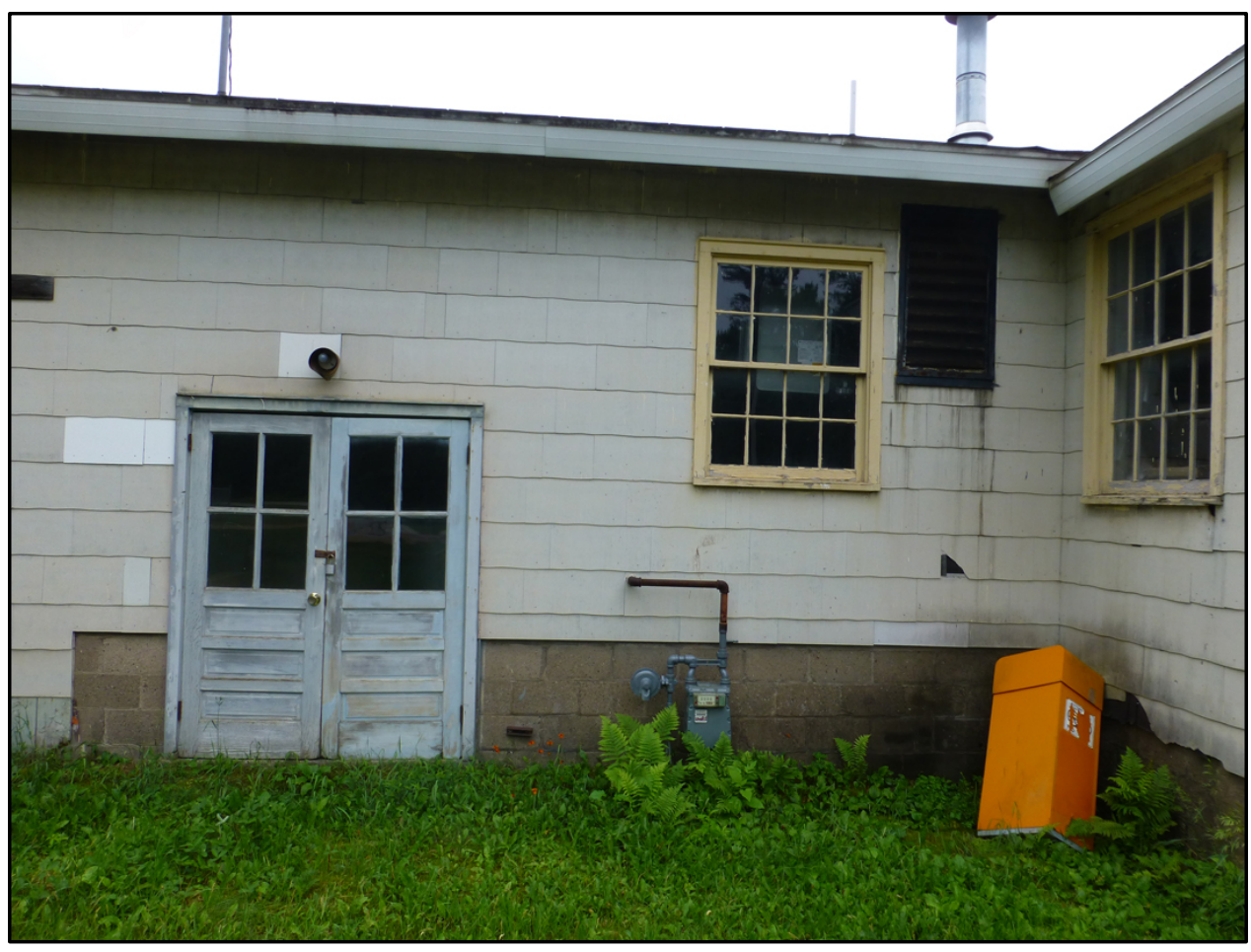

Figure 78. Looking at concrete block from exterior on the south side of Bldg. 550, 2018.

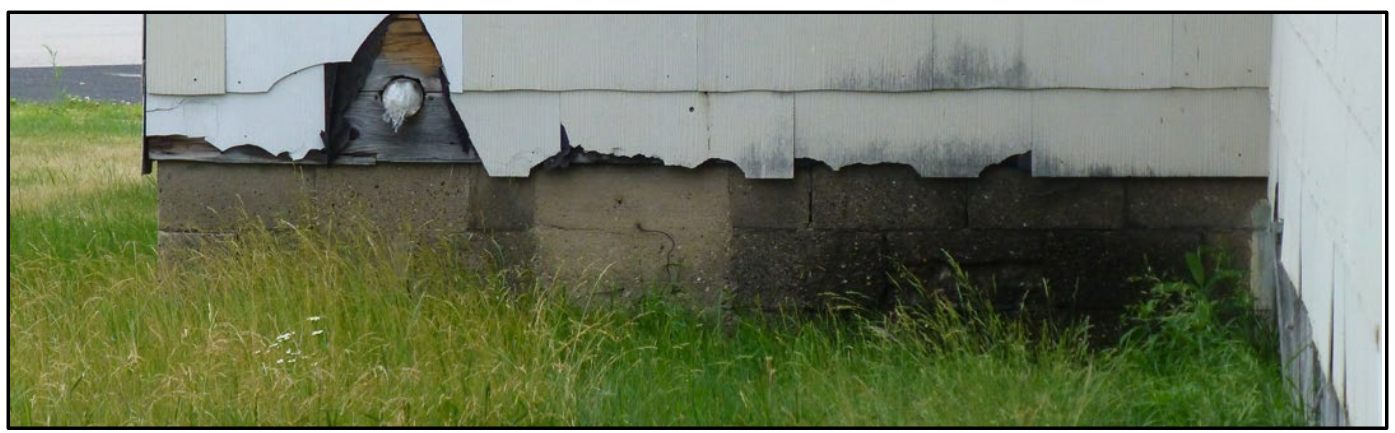

In the 2018 photo shown in Figure 79, cracking and chipping in the original concrete block. Over time, the mortar in stone masonry decays and crumbles away. This could allow water, air, or pests to seep into the building's interior.

Figure 79. Looking at concrete block from interior, 2018.

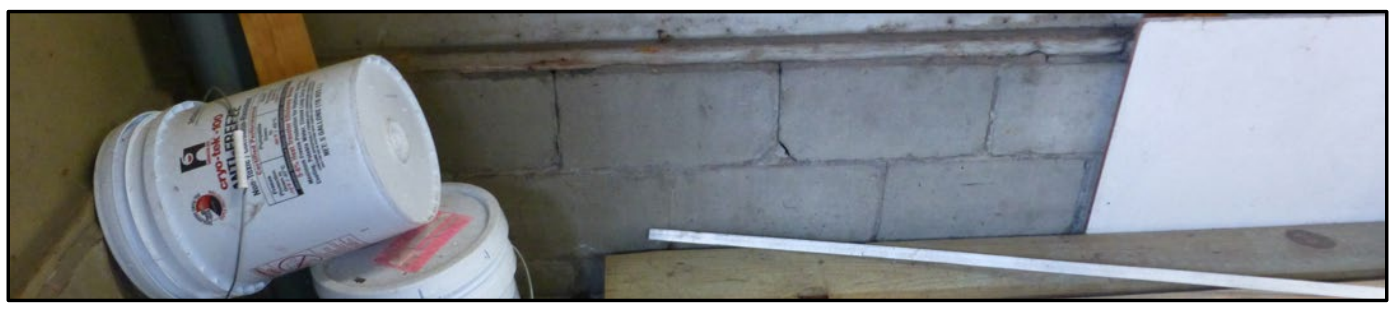




\subsection{Treatment measures}

The two uses of concrete in Bldg. 550 is the poured floors and the concrete block. The condition of concrete can change drastically in time due to the weather and the settling of a structure. Below is information discussing repair methods of aged concrete. 


\subsubsection{Types of cracks in concrete and typical causes, ${ }^{21} 2016$ (GSA.gov).}

GSA U.S. General Services Administration

\section{Types of Cracks in Concrete and Typical Causes}

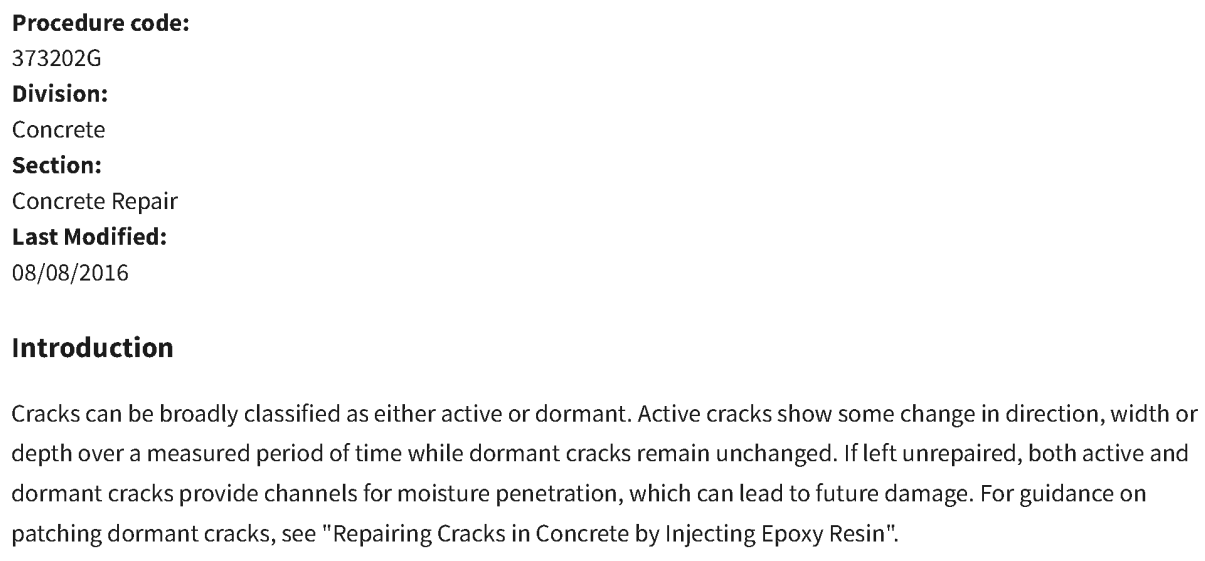

References

- "Identifying Cracks in Slabs". Concrete Construction. Web. July 31, 2014.

- Interval, V. "Concrete Cracks: An Overview of Types of Cracking/Deterioration and Their Implications." Wikispaces Classroom. Web.

- Portland Cement Association. "IS-177 Concrete Slab Surface Defects- Causes, Prevention, Repair". Concrete Information. 2001.

\section{Types of Cracks}

The severity of a crack can be characterized in terms of its direction, width, and depth; cracks may be longitudinal, transverse, vertical, diagonal or random. Different risks for cracking exist for cured versus uncured concrete, and for reinforced concrete. Breakages occur through thermal, chemical or mechanical processes causing shrinkage, expansion or flexural stress. Below is a list of types of concrete cracks, and some of their possible causes:

${ }^{21}$ Retrieved from: https://www.gsa.gov/real-estate/historic-preservation/historic-preservation-policytools/preservation-tools-resources/technical-documents. 
A. Plastic-shrinkage cracking: Cracks that run to the mid-depth of the concrete, are distributed across the surface unevenly, and are usually short in length.

- Most often occurs while concrete is curing, due to the surface of the concrete drying too rapidly relative to the concrete below.

B. Crazing/Map cracking/Checking: A web of fine, shallow cracks across the surface of the concrete.

- Also occur during curing due to the surface of concrete drying faster than the interior concrete, but the surface drying occurs at a lesser depth.

- Because this type of cracking is limited to the surface, it does not usually pose serious structural problems.

C. Hairline cracking: Very thin but deep cracks.

- Due to settlement of the concrete while it is curing.

- Due to their depth, these cracks can allow for more serious cracking once the concrete is hardened.

D. Pop-Outs: Conical depressions in the concrete surface

- Occurs when a piece of aggregate near the concrete surface is particularly absorbent, causing it to expand and pop out of the surface of the concrete.

E. Scaling: Small pock marks in the concrete surface, exposing aggregate underneath.

- Once cured, if concrete does have an adequate finish to prevent water penetration, water that seeps into the concrete will expand when it freezes, pushing off pieces of the concrete surface.

- Scaling can also be caused by delamination, which occurs when too much water (due to insufficient curing) or air (due to insufficient vibrating) remains in the concrete when it is finished. The water and air rise to the top and form pockets below the surface. These pockets may form blisters or which may break open to create scaling.

F. Spalling: Surface depressions that are larger and deeper than scaling, often linear when following the length of a rebar.

- Also caused by pressure from under the surface of the concrete.

- Most often occur due to improperly constructed joints or the corrosion of rebar in the concrete

- Corrosion creates pressure as rust forms, which can push away large chunks of concrete, and expose the corroded metal below.

- Spalling that exposes corroded metal can be particularly problematic because the corrosion is likely to accelerate due to exposure to air and water.

G. D-Cracking: Cracks that runs roughly parallel or stem from a concrete joint and are deeper than surface cracks. 
- Due to moisture infiltration at the joint.

H. Offset cracking: Cracks where the concrete on one side of the crack is lower than the concrete on the other side

- Due to uneven surfaces below the concrete, such as subgrade settlement or pressure from objects such as tree roots, previously-placed concrete, or rebar.

I. Diagonal corner cracking: Cracks that run from one joint to its perpendicular joint at the corner of a slab

- The corners of concrete slabs can be prone to curling(due to differences in temperature at different depths in the curing concrete) or warping (due to differences in moisture evaporation at different depths in the curing concrete). The dryer or colder level of concrete will shrink more and create cracks as the concrete dries.

- Because the warped or curled-up corners often have some empty space below them, they are also prone to cracking after curing due to weight overload causing the corner to snap downward into the empty space.

Last Reviewed: 2017-09-2ধ 
7.4.2 Removing and replacing deteriorated concrete paving, ${ }^{22} 2015$ (GSA.gov).

\title{
Removing And Replacing Deteriorated Concrete Paving
}

\author{
Procedure code: \\ 3732035 \\ Source: \\ Hsr - Icc Bldg/Wash, Dc - Grier, Brown, Renfrow Architects \\ Division: \\ Concrete \\ Section: \\ Concrete Repair \\ Last Modified: \\ 06/09/2015
}

PART 1---GENERAL

1.01 SUMMARY

A. This procedure includes guidance on replacing damaged sections of concrete paving.

B. See 01100-07-S for general project guidelines to be reviewed along with this procedure. These guidelines cover the following sections:

1. Safety Precautions

2. Historic Structures Precautions

3. Submittals

4. Quality Assurance

5. Delivery, Storage and Handling

6. Project/Site Conditions

7. Sequencing and Scheduling

8. General Protection (Surface and Surrounding) These guidelines should be reviewed prior to performing this procedure and should be followed, when applicable, along with recommendations from the Regional Historic Preservation Officer (RHPO).

\subsection{PROJECT/SITE CONDITIONS}

A. Environmental Requirements: Maintain a constant temperature of 45 degrees F. or above for at least 48 hours prior to placing concrete.

22 Retrieved from: https://www.gsa.gov/real-estate/historic-preservation/historic-preservation-policytools/preservation-tools-resources/technical-documents. 


\section{PART 2---PRODUCTS}

\subsection{MATERIALS}
A. Portland cement
B. Aggregate
C. Clean, potable water
D. Polyethylene moisture barrier
E. Moisture-retaining cover such as waterproof paper, polyethylene film or polyethylene-coated burlap
F. Expansion joint material such as cork strips

\subsection{EQUIPMENT}
A. Spade
B. Mechanical vibrating equipment
C. Stiff bristle brushes
D. Straight edge
E. Bull float or darby

\section{PART 3---EXECUTION}

\subsection{ERECTION, INSTALLATION, APPLICATION}

A. Remove deteriorated sections of paving to edge of sound pavers and down to structural slab.

B. Remove dirt and debris from surface to receive new concrete.

C. Cut and fit polyethylene moisture barrier to cover the area to receive new concrete.

D. Position expansion joint material at edges of floor slab as required.

E. Lay concrete in layers. DO NOT allow the concrete to harden before placing the next layer.

1. Consolidate concrete while laying by hand spading, rodding or tamping along with using mechanical vibrating equipment.

2. Work concrete thoroughly into corners.

F. Fill area flush with adjacent sound concrete and level with a straight edge.

G. Smooth the surface with a bull float or darby to eliminate humps or hollows.

H. Finish surface to match adjacent paving.

\subsection{ADJUSTING/CLEANING}

A. Protect newly placed concrete from excessive hot and cold temperatures. Cover with moisture-retaining cover while curing. Lap ends at least 3 inches and seal with waterproof tape or adhesive.

B. Allow to cure for a minimum of 7 days. 


\title{
7.4.3 Patching spalled concrete, 2017 (GSA.gov).
}

\section{Patching Spalled Concrete}

\author{
Procedure code: \\ 3732045 \\ Source: \\ National Capitol Region Specification - Agriculture Building \\ Division: \\ Concrete \\ Section: \\ Concrete Repair \\ Last Modified: \\ 07/07/2017
}

\section{PART 1---GENERAL}

\subsection{SUMMARY}

A. This procedure includes guidance on patching spalls and holes in concrete with a cementitious patching material.

B. See 01100-07-S for general project guidelines to be reviewed along with this procedure. These guidelines cover the following sections:

1. Safety Precautions

2. Historic Structures Precautions

3. Submittals

4. Quality Assurance

5. Delivery, Storage and Handling

6. Project/Site Conditions

7. Sequencing and Scheduling

8. General Protection (Surface and Surrounding)

These guidelines should be reviewed prior to performing this procedure and should be followed, when applicable, along with recommendations from the Regional Historic Preservation Officer (RHPO).

\subsection{QUALITY ASSURANCE}

A. Masonry and Concrete Repair: Prepare sample panels of size indicated for each type of masonry material indicated to be patched, rebuilt or replaced. 


\section{PART 2---PRODUCTS}

\subsection{MANUFACTURERS}

A. Sika Corporation usa.skie.com

B. General Polymers, www.generalpolymers.com

C. BASF Master Builders Solutions, www.master-builders-solutions-basf.us

D. Euclid Chemical, www.euclidchemical.com

\subsection{MATERIALS}

A. Concrete Patching Material: One component, earlystrength, cementitious patching material "Sike Repair 222" (Sika Corporation); "TPM 723" (General Polymers); (Master Builders), or approved equal.

B. Water: Clean, free of oils, acids, alkalis and organic matter.

\subsection{EQUIPMENT}
A. Trowels
B. Chisels
C. Stiff bristle brushes (non-metallic)

\section{PART 3---EXECUTION}

\subsection{PREPARATION}

A. Protection:

1. Protect persons, motor vehicles, surrounding surfaces of building whose masonry surfaces are being restored, building site, and surrounding buildings from injury resulting from masonry restoration work.

2. Erect temporary protection covers over pedestrian walkways and at points of entrance and exit for persons and vehicles which must remain in operation during course of masonry restoration work.

3. Contractor shall test those areaway drains, window well drains, etc., which will be used to assure that drains are functioning properly prior to performing masonry restoration operations in those areas. The Contractor shall report immediately to the Construction Engineer the location of drains which are found to be stopped up or blocked.

4. Prevent grout or mortar used in repointing and repair work from staining face of surrounding masonry and other surfaces. Remove immediately grout and mortar in contact with exposed masonry and other surfaces.

5. Protect sills, ledges, windows, and projections from patching material droppings.

\subsection{ERECTION, INSTALLATION, APPLICATION}

A. Remove deteriorated concrete at spalls to sound material. Grind, chisel or saw cut 1" deep undercut around perimeter of patch. Clean with compressed air. Thoroughly remove any concrete showing traces of oils or grease.

B. Thoroughly wet patched area prior to casting concrete patching material. If cement patching material manufacturer recommends a different procedure, such procedure is to be followed and executed in accordance with published instructions and in accordance with approved test patch.

C. Install cement patching material in strict accordance with manufacturer's published instructions.

D. Finish surface to match surface being patched, by grinding, troweling, sacking, or brushing.

\subsection{ADJUSTING/CLEANING}

A. After mortar has fully hardened, thoroughly clean exposed masonry surfaces of excess mortar and foreign matter using stiff nylon or bristle brushes and clean water, spray applied at low pressure.

B. Use of metal scrapers or brushes will not be permitted.

C. Use of acid or alkali cleaning agents will not be permitted. 


\title{
7.4.4 Treating dusting of concrete floors, ${ }^{23} 2017$ (GSA.gov).
}

\section{Treating Dusting of Concrete Floors}

\author{
Procedure code: \\ 3710025 \\ Division: \\ Concrete \\ Section: \\ Concrete Cleaning \\ Last Modified: \\ 02/21/2017
}

\section{PART 1---GENERAL}

\subsection{SUMMARY}

A. This specification explains how to treat dusting of concrete floors. Dusting is a powder that develops at the surface of hardened concrete, and is composed of disintegrated concrete.

B. The treatment described in this procedure includes mopping with a manufactured liquid treatment (typically a mixture of fluosilicate and water).

1. Fluosilicate makes the concrete surface harder and more impermeable.

2. This treatment also improves the concrete surface for the application of oil base paints and varnishes.

C. Other treatment options for dealing with dusting of concrete floors include:

1. Removal of the dusting layer down to a hard durable layer if the floor elevation can be modified, or;

2. Removal of the dusting layer down to a hard durable layer and applying a topping coating to bring the floor surface up to the prior level.

D. Safety Precautions:

1. Do not save unused portions of stain-removal materials.

2. Do not store any chemicals in unmarked containers.

3. Excellent ventilation must be provided wherever any solvent is used. Use respirators with solvent filters.

23 Retrieved from: https://www.gsa.gov/real-estate/historic-preservation/historic-preservation-policytools/preservation-tools-resources/technical-documents. 
4. No use of organic solvents indoors should be allowed without substantial air movement. Use only spark-proof fans near operations involving flammable liquids.

5. Provide adequate clothing and protective gear where the chemicals are indicated to be dangerous.

6. Have available antidote and accident treatment chemicals where noted.

E. Read "General Project Guidelines" along with this specification. These guidelines should be reviewed prior to performing this procedure and should be followed, when applicable, along with recommendations from the Regional Historic Preservation Officer (RHPO). The guidelines cover the following sections:
1. Safety Precautions
2. Historic Structures Precautions
3. Submittals
4. Quality Assurance
5. Delivery, Storage and Handling
6. Project/Site Conditions
7. Sequencing and Scheduling
8. General Protection (Surface and Surrounding)

\section{PART 2---PRODUCTS}

\subsection{MANUFACTURERS}

Manufactured concrete chemical products are available such as L\&M Seal Hard (made by Laticrete International, Inc.) or approved equal.

\subsection{MATERIALS}

NOTE: Chemical products are sometimes sold under a common name. This usually means that the substance is not as pure as the same chemical sold under its chemical name. The grade of purity of common name substances, however, is usually adequate for stain removal work, and these products should be purchased when available, as they tend to be less expensive. Common names are indicated below by an asterisk $\left({ }^{\star}\right)$.

A. Fluosilicate:

1. Magnesium Fluosilicate:

a. Other chemical or common names include Magnesium silicofluoride.

b. Potential Hazards: TOXIC.

c. Available from construction specialties distributor (often sold under manufacturer's brand name; the chemical name may appear on the label). 
2. Zinc Fluosilicate:

a. Other chemical or common names include Zinc silicofluoride.

b. Available from construction services distributor (often sold under a manufacturer's brand name; the chemical name may appear on the label).

B. Clean, potable water.

\subsection{EQUIPMENT}

A. Garden hose and pneumatic spray nozzle.

B. Stiff bristle brushes (non-metallic).

C. Mopping Tools:

1. Wet mop.

2. Mop bucket.

3. Wringer.

4. Sponge mop and squeegee.

\section{PART 3---EXECUTION}

\subsection{PREPARATION}

Protection: Provide adequate wash solutions (e.g., water, soap and towels) before starting the job.

\subsection{ERECTION, INSTALLATION, APPLICATION}

NOTE: Do not try more than one treatment on a given area unless the chemicals used from prior treatment have been washed away.

A. Make sure the floor is clean before you begin.

B. Apply an appropriate manufacturer-supplied concrete hardening treatment and follow the manufacturer's guidance on the application.

Or, if a contractor, this prepared treatment is proposed:

C. Mix 1 pound of fluosilicate (a mixture of $20 \%$ zinc fluosilicate and $80 \%$ magnesium fluosilicate) with 1 gallon of water.

D. Mop the mixture generously on the concrete surface and allow to dry.

E. If additional coats are necessary, mix 2 pounds of fluosilicate (20/80 as in $3.02 \mathrm{C}$ above) in 1 gallon of water and apply as described in $3.02 \mathrm{~B}$ above. Two or three coats may be necessary. 
F. When the last coat has dried, thoroughly rinse the surface with clean, clear water and scrub with a stiff bristle brush to remove any crystals that have formed. 


\title{
7.4.5 Removing surface dirt from concrete, ${ }^{24} 2016$ (GSA.gov).
}

\section{Removing Surface Dirt From Concrete}

\author{
Procedure code: \\ 3710155 \\ Source: \\ Hstrc Concrete: Investigation \& Rpr/Pre-Conf Training - 1989 \\ Division: \\ Concrete \\ Section: \\ Concrete Cleaning \\ Last Modified: \\ $08 / 02 / 2016$
}

PREFACE: The cleaning or removal of stains from concrete may involve the use of liquids, detergents or solvents which may run off on $\backslash$ adjacent material, discolor the concrete or drive the stains deeper into porous concrete. Use the products and techniques described here only for the combinations of dirt/stain and concrete specified.

PART 1---GENERAL

1.01 SUMMARY

A. This procedure includes guidance on removing dirt from concrete using a detergent, chemical solvent or steam.

B. Dirt encompasses deposits of almost any material in a location where it's not wanted, but it usually includes fine, dark-colored solid particles, often surrounded by some kind of oily film. It is particularly troublesome on architectural and decorative concrete, including exposed aggregate surfaces.

C. Safety Precautions:

1. DO NOT save unused portions of stain-removal materials.

2. DO NOT store any chemicals in unmarked containers.

3. EXCELLENT VENTILATION MUST BE PROVIDED WHEREVER ANY SOLVENT IS USED. USE RESPIRATORS WITH SOLVENT FILTERS.

4. Whenever acid is used, the surface should be thoroughly rinsed with water as soon as its action has been adequate. Otherwise it will continue etching the concrete even though the stain is gone.

5. Provide adequate clothing and protective gear where the chemicals are indicated to be dangerous.

6. Have available antidote and accident treatment chemicals where noted.

D. See "General Project Guidelines" for general project guidelines to be reviewed along with this procedure. These guidelines cover the following sections:

1. Safety Precautions

2. Historic Structures Precautions

3. Submittals

4. Quality Assurance

5. Delivery, Storage and Handling

6. Project/Site Conditions

${ }^{24}$ Retrieved from: https://www.gsa.gov/real-estate/historic-preservation/historic-preservation-policytools/preservation-tools-resources/technical-documents. 
7. Sequencing and Scheduling

8. General Protection (Surface and Surrounding)

These guidelines should be reviewed prior to performing this procedure and should be followed, when applicable, along with recommendations from the Regional Historic Preservation Officer (RHPO).

PART 2---PRODUCTS

2.01 MATERIALS

NOTE: Chemical products are sometimes sold under a common name. This usually means that the substance is not as pure as the same chemical sold under its chemical name. The grade of purity of common name substances, however, is usually adequate for stain removal work, and these products should be purchased when available, as they tend to be less expensive. Common names are indicated below by an asterisk $\left({ }^{*}\right)$.

A. Hydrochloric Acid:

1. A strong corrosive irritating acid.

2. Other chemical or common names include Chlorhydric acid; Hydrogen chloride; Muriatic acid*; Marine

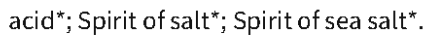

3. Available from chemical supply house, drugstore, hardware store.

B. Detergent:

1. CAUTION: SOME DETERGENTS CONTAIN AMMONIA AND MAY REACT VIGOROUSLY WITH HYDROCHLORIC ACID.

C. Clean, potable water

D. Clean white cloths or towels

\subsection{EQUIPMENT}

A. Steam cleaning equipment

B. Stiff bristle brushes (non-metallic)

PART 3---EXECUTION

3.01 PREPARATION

A. Protection:

1. Provide adequate wash solutions (i.e. water, soap and towels) before starting the job.

2. Whenever acid is used, the surface should be thoroughly rinsed with water as soon as its action has been adequate. Otherwise it will continue etching the concrete even though the stain is gone.

\subsection{ERECTION, INSTALLATION, APPLICATION}

\section{NOTE: DO NOT TRY MORE THAN ONE TREATMENT ON A GIVEN AREA UNLESS THE CHEMICALS USED FROM PRIOR} TREATMENT HAVE BEEN WASHED AWAY.

A. Brush affected area with water and strong detergent

B. Rinse the area thoroughly with clean, clear water and blot the surface dry with clean towels.

C. Repeat the treatment as necessary until the desired level of cleanliness is achieved. -OR-

D. Mix 1 part hydrochloric acid in 19 parts water.

E. Scrub the concrete surface with this solution. NOTE: THIS IS A STRONG METHOD AND MAY ROUGHEN THE CONCRETE.

F. Rinse the area thoroughly with clean, clear water, blot the surface dry with clean towels.

$\mathrm{G}$. Repeat the treatment as necessary until the desired level of cleanliness is achieved. -OR-

$\mathrm{H}$. Steam cleaning is generally effective and may be used in combination with proprietary materials, such as detergents for dirt removal.

I. If there is oil present in the dirt, follow the procedure described for removing lubricating oil, see 03710-31-R "Poulticing Lubricating and Petroleum Oil Stains From Concrete". 


\title{
7.4.6 Poulticing iron rust stains from concrete, 2016 (GSA.gov).
}

\author{
GSA U.S. General Services Administration
}

Poulticing Iron Rust Stains From Concrete

\author{
Procedure code: \\ 3710265 \\ Source: \\ Hstrc Concrete: Investigation \& Rpr/Pre-Conf Training - 1989 \\ Division: \\ Concrete \\ Section: \\ Concrete Cleaning \\ Last Modified: \\ 08/04/2016
}

PREFACE: The cleaning or removal of stains from concrete may involve the use of liquids, detergents or solvents which may run off on adjacent material, discolor the concrete or drive the stains deeper into porous concrete. Use the products and techniques described here only for the combinations of dirt/stain and concrete specified.

PART 1---GENERAL

1.01 SUMMARY

A. This procedure includes guidance on removing both surface and penetrating rust stains from concrete by poulticing with chemical solvents.

B. Stains of a rust color on concrete are usually caused by rusting of steel in or on concrete, from use of curing water that contains iron in solution or from pyrites (iron sulfide particles) occasionally found in aggregate.

C. Safety Precautions:

1. DO NOT save unused portions of stain-removal materials.

2. DO NOT store any chemicals in unmarked containers.

3. EXCELLENT VENTILATION MUST BE PROVIDED WHEREVER ANY SOLVENT IS USED. USE RESPIRATORS WITH SOLVENT FILTERS.

4. No use of organic solvents indoors should be allowed without substantial air movement. Use only spark-proof fans near operations involving flammable liquids.

5. Provide adequate clothing and protective gear where the chemicals are indicated to be dangerous.

6. Have available antidote and accident treatment chemicals where noted.

D. See "General Project Guidelines" for general project guidelines to be reviewed along with this procedure. These guidelines cover the following sections:

1. Safety Precautions

2. Historic Structures Precautions

3. Submittals

4. Quality Assurance 
5. Delivery, Storage and Handling

6. Project/Site Conditions

7. Sequencing and Scheduling

8. General Protection (Surface and Surrounding)

These guidelines should be reviewed prior to performing this procedure and should be followed, when applicable, along with recommendations from the Regional Historic Preservation Officer (RHPO).

\section{PART 2---PRODUCTS}

\subsection{MATERIALS}

NOTE: Chemical products are sometimes sold under a common name. This usually means that the substance is not as pure as the same chemical sold under its chemical name. The grade of purity of common name substances, however, is usually adequate for stain removal work, and these products should be purchased when available, as they tend to be less expensive. Common names are indicated below by an asterisk (*).

A. For Light Shallow Stains:

1. Oxalic Acid $(\mathrm{COOH}) 2$ or $(\mathrm{H} 2 \mathrm{C} 2 \mathrm{O} 4)$ :

a. A poisonous strong acid that occurs in various plants as oxalates and is used especially as a bleaching or cleaning agent and in making dyes.

b. Other chemical or common names include Ethanedioic acid.

c. Potential Hazards: TOXIC; CORROSIVE TO CONCRETE, STEEL, WOOD OR GLASS.

d. Available from chemical supply house, dry cleaning supply distributor, drugstore or pharmaceutical supply distributor, hardware store, or photographic supply distributor (not camera shop). (Often sold under a manufacturer's brand name; the chemical name may appear on the label.)

2. Ammonium Hydrogen Fluoride: USE EXTREME CAUTION WITH THIS MATERIAL.

a. Other chemical or common names include Acid Ammonium fluoride; Ammonium bifluoride.

b. Potential Hazards: TOXIC; CAUSTIC TO FLESH; CORROSIVE TO CONCRETE, STEEL, WOOD OR GLASS; FLAMMABLE.

c. Available from chemical supply house or dairy supply distributor.

d. USE EXTREME CAUTION WITH THIS MATERIAL.

B. For Deep Penetrating Stains:

1. Sodium Citrate (appears like enlarged salt granules):

a. Other chemical or common names include Citrate of soda*.

b. Available from chemical supply house, drug store or pharmaceutical supply distributor.

2. Glycerol:

a. Other chemical or common names include Glycerine; Glyceryl hydroxide; Glycyl alcohol; 1,2,3propanetriol; Propenyl alcohol.

b. Potential Hazards: FLAMMABLE.

c. Available from chemical supply house, drug store or hardware store.

3. Hydrogen Peroxide (H202):

a. An unstable compound used especially as an oxidizing and bleaching agent, an antiseptic, and a propellant.

b. Other chemical or common names include Peroxide of hydrogen*; Solution of hydrogen dioxide*; Superoxol*; (hydrogen peroxide is commonly sold as a 3\% solution; Superoxol is a $30 \%$ solution; Superoxol causes flesh burns; $3 \%$ hydrogen peroxide does not).

c. Potential Hazards: TOXIC (when concentrated); CAUSTIC TO FLESH; FLAMMABLE (in high concentration).

d. Available from chemical supply house, drugstore, pharmaceutical supply distributor, or hardware store.

4. Sodium Thiosulfate - white sal or "hypo" of photographic fixing agent (NA2S2O3):

a. A hygroscopic crystalline salt used especially as a photographic fixing agent and a reducing or bleaching agent

b. Other chemical or common names include Sodium hydrosulfite; Sodium Hyposulfite; Sodium subsulfite; Antichlor*; Hypo*; Hyposulfite of soda*.

c. Potential Hazards: TOXIC; CORROSIVE TO CONCRETE, STEEL, WOOD OR GLASS. 
C. Soap Powder

d. Available from chemical supply house, dry cleaning supply distributor, drugstore or pharmaceutical supply distributor, photographic supply distributor (not camera shop), or water and sanitation supply distributor.

D. Filler material such as diatomaceous earth, talc or cotton wadding

E. Mineral water

F. Plastic sheeting

G. Clean dry towels for blotting the area after treatment

H. Masking tape

I. Clean, potable water

J. Accessible source of water, soap and towels for washing and rinsing in case of emergencies associated with the use of chemicals

\subsection{EQUIPMENT}

A. Stiff bristle brushes (non-metallic)

B. Glass or ceramic container for mixing the solution

C. Wooden utensil for stirring the ingredients

D. Wood or plastic spatula

PART 3---EXECUTION

\subsection{PREPARATION}

A. Protection:

1. Provide adequate wash solutions (i.e. water, soap and towels) before starting the job.

2. Whenever acid is used, the surface should be thoroughly rinsed with water as soon as its action has been adequate. Otherwise it will continue etching the concrete even though the stain is gone.

\subsection{ERECTION, INSTALLATION, APPLICATION}

NOTE: DO NOT TRY MORE THAN ONE TREATMENT ON A GIVEN AREA UNLESS THE CHEMICALS USED FROM PRIOR TREATMENT HAVE BEEN WASHED AWAY.

A. For Light Surface Stains:

1. Mix 1 pound oxalic acid in 1 gallon of water. CAUTION: OXALIC ACID IS TOXIC. OBSERVE WARNINGS ON THE LABEL.

2. Swab the stained area with the solution.

3. To quicken the stain removal action, add $1 / 2$ pound of ammonium acid fluoride to the solution. CAUTION: AMMONIUM ACID FLUORIDE GENERATES HYDROFLUORIC ACID, WHICH IS HIGHLY AGGRESSIVE AND TOXIC TO SKIN, EYES AND MUCOUS MEMBRANES. THE EFFECTS ARE FAIRLY LONGLASTING, SO THIS COMPOUND SHOULD BE HANDLED WITH EVEN MORE CARE THAN MOST MINERAL ACIDS.

4. Allow the solution to sit on the stained concrete for 2 or 3 hours.

5. Thoroughly rinse the surface by scrubbing with a stiff bristle brush and clean, clear water.

6. Repeat the treatment as necessary to achieve the desired level of cleanliness.

B. For Deep Penetrating Stains:

1. Dissolve 11 ounces by weight of sodium citrate in 2 quarts of lukewarm water and add 2 quarts and 12 ounces of glycerol.

2. Mix the solvent solution above with diatomaceous earth or talc to form a thick paste having the consistency of oatmeal.

3. Thoroughly wet the concrete surface to be treated with clean, clear water.

4. Apply the poultice to the stained area using a wood or plastic spatula and allow to dry. Be sure to spread the poultice well beyond the stained area. The liquid portion of the paste will migrate into the concrete where it will dissolve some of the staining material. Then the liquid will gradually move back beyond the concrete surface and into the poultice, where it will evaporate, leaving the dissolved staining material in the poultice.

5. When the poultice has dried, brush or scrape it off with a wooden scraper. 
6. Using a stiff bristle brush, scrub the surface with scouring powder and clean water to remove any residual staining.

7. Thoroughly rinse the area with clean, clear water and allow to dry.

8. Repeat the process as necessary to sufficiently remove the stain.

$-\mathrm{OR}-$

NOTE: THIS METHOD REQUIRES GOOD VENTILATION TO REMOVE THE ACRID FUMES OF SULFUR DIOXIDE GIVEN OFF.

9. Dissolve 22 ounces by weight of sodium citrate in 1 gallon of lukewarm water.

10. Saturate a bandage of cotton wadding in the solvent solution and apply the bandage for 30 minutes, or brush the solution on the surface every 5 or 10 minutes.

11. For stains on horizontal surfaces:

a. Sprinkle a thin layer of sodium thiosulfate over the surface and moisten it with a light mist of water from a spray nozzle.

b. Cover the treated area with a poultice made with diatomaceous earth or talc moistened with water.

12. For stains on vertical surfaces:

a. Place a poultice of diatomaceous earth or talc mixed with water on a trowel.

b. Sprinkle sodium thiosulfate crystals on the poultice and moisten slightly.

c. Trowel the poultice mixture onto the surface so that the crystals are in direct contact with the stained surface.

d. NOTE: If the thiosulfate crystals turn the brown stains to black, brush apply a diluted solution of hydrogen peroxide to the stained area and repeat the thiosulfate treatment.

13. After 1 hour, remove the poultice. Repeat the treatment with fresh materials as necessary to achieve the desired level of cleanliness.

14. Scrub the surface thoroughly with clean, clear water and apply another treatment of the sodium citrate solution to prevent the stain from coming 
7.4.7 Concrete block: characteristics, uses, and problems, ${ }^{25} 2017$ (GSA.gov).

囬 An official website of the United States government

GSA U.S. General Services Administration

Concrete Block: Characteristics, Uses And Problems

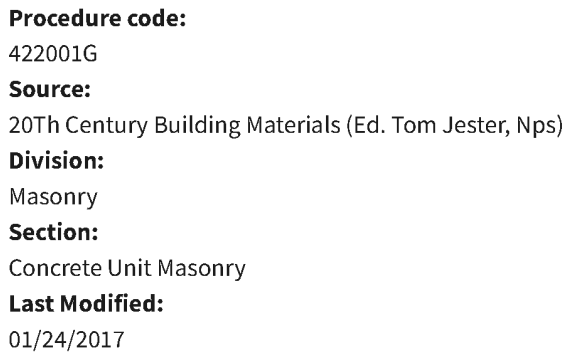

This standard includes general information on the characteristics and common uses of concrete block and identifies typical problems associated with this material along with common causes of its deterioration.

Reference: National Park Service Preservation Brief \#15: Preservation of Historic Concrete

https://www.nps.gov/tps/how-to-preserve/briefs/15-concrete.htm

\section{Characteristics of Concrete Block:}

- Made from a mixture of Portland cement, blended cement, various types of aggregates, and water.

- Also referred to as concrete masonry units (CMU).

- Advantages:

- Inexpensive,

- Lightweight

- Durable,

- Easy to install

- Fireproof

- Low maintenance

- Could be ornamented

- Face plates were used to create a variety of surface finishes, including cobblestone, brick, ashlar and rockface (the most common type); more decorative finishes included designs of scrolls, wreaths and roping.

25 Retrieved from: https://www.gsa.gov/real-estate/historic-preservation/historic-preservation-policytools/preservation-tools-resources/technical-documents. 
- Typical size manufactured is nominally for a stretcher block 8 by 8 by 16 inches; this was the standard size manufactured by 1930 (actual dimensions 8 by $73 / 4$ by $153 / 4$ inches).

- Typical size manufactured is nominally for a stretcher block 8 by 8 by 16 inches; this was the standard size manufactured by 1930 (actual dimensions 8 by $73 / 4$ by $153 / 4$ inches).

- They may be solid or hollow with two or three cores for such stretcher blocks; various other types of standard shapes are also often available and one should consult the local market to determine availability. Block ends may be flat or flanged.

- Compressive strength and fire resistance of the each block is dependent upon the block's configuration.

- Lightweight aggregates were introduced around 1917 and cinder blocks were patented.

- Advantages of using cinder blocks included its strength, ability to receive nails and ease of installation.

- Lightweight aggregates were either natural materials, by-products or manufactured.

- Natural aggregate materials included pumice.

- By-products aggregate materials included cinders and slag; Pottsco or Celocrete is one example of slag product used around 1930 in the manufacture of blocks; Waylite is another example introduced in the late 1930s.

- Manufactured aggregate materials included expanded shale, clay and slate; Haydite is one example of an expanded shale product used in the early 1920s in the manufacture of blocks.

\section{Typical Uses}

- Typical historical uses for concrete block include:

- Foundation walls - typically rockfaced.

- Basement walls.

- Partition walls - usually plainfaced.

- Exterior walls - usually plainfaced and then often covered with stucco.

- Most concrete block was used as a back-up material or for cavity wall construction.

- Coatings are often are applied to concrete block in order to prevent water penetration; some of these include Portland cement paints, latex paints, oil- and rubber-based coatings, epoxy coatings, alkyd paints, urethanes and silicones; a single type may be selected for a specific function including its water resistance; other factors to consider might also include its resistance to ultraviolet rays, its breathability, its resistance to alkalis, and its colorationor visual appearance when applied to the block.

\section{Natural or Inherent Problems}

- Cracking: Often due to shrinkage of the concrete or movement of the wall.

- Efflorescence: Occurs when accumulations of salt are carried to the surface by water migrating through the masonry.

- Staining: Staining may appear in many forms, including dirt build-up, metallic staining or painted Rising Damp: When ground water enters the wall from the base and migrates upward.

\section{Vandalism and other Human-induced Problems}

- Spalling: May be caused by the composition of the concrete mixture, prolonged exposure to water which has infiltrated the wall, or mechanical failure. 
7.4.8 Patching chips and cracks in ornamental concrete block, ${ }^{26} 2017$ (GSA.gov).

\title{
Patching Chips And Cracks In Ornamental Concrete Block
}

\author{
Procedure code: \\ 422003S \\ Source: \\ Developed For Hspg (Nps - Sero) \\ Division: \\ Masonry \\ Section: \\ Concrete Unit Masonry \\ Last Modified: \\ $04 / 17 / 2017$
}

PART 1---GENERAL

1.01 SUMMARY

A. This procedure includes guidance on repairing chips, cracks or holes in ornamental concrete block. Small areas may be patched using a mortar mixture; larger areas may require pins and mortar for additional reinforcement.

B. See 01100-07-S for general project guidelines to be reviewed along with this procedure. These guidelines cover the following sections:

1. Safety Precautions

2. Historic Structures Precautions

3. Submittals

4. Quality Assurance

5. Delivery, Storage and Handling

6. Project/Site Conditions

7. Sequencing and Scheduling

8. General Protection (Surface and Surrounding)

These guidelines should be reviewed prior to performing this procedure and should be followed, when applicable, along with recommendations from the Regional Historic Preservation Officer (RHPO).

1.02 REFERENCES

A. American Society for Testing and Materials (ASTM) www.astm.org

1.03 PROJECT/SITE CONDITIONS

A. Environmental Requirements:

26 Retrieved from: https://www.gsa.gov/real-estate/historic-preservation/historic-preservation-policytools/preservation-tools-resources/technical-documents. 
1. Check manufacturer's literature for precautions and effects of products and procedures on adjacent building materials, components, and especially vegetation; Take appropriate protective measures as necessary.

2. Wet Weather: Do not apply or mix mortar on outside surfaces with standing water or outside during rain.

3. Do not proceed with patching under adverse weather conditions, or when temperatures are below or above manufacturer's recommended limitations for installation; Proceed with the work only when forecasted weather conditions are favorable for proper cure.

4. Cold Weather, winter construction is not allowed without consent of Regional Architect; Winter construction is defined as any time when surface temperature of masonry is below 50 degrees $\mathrm{F}$. or air temperature is predicted to be below 40 degrees F. within 48 hours; Heat mortar materials to above 50 degrees $F$. if necessary.

5. Work must not be done at temperatures above 80 degrees F. unless shading and water-misted burlap over new work is provided. Mortar mixing should be done only in the shade; cover mortar in hot weather to reduce evaporation. Pointing work should be done in the shade. Work around the building during the day so that the fresh work will be shielded from direct sunlight to reduce evaporation rate.

\section{PART 2---PRODUCTS}

\subsection{MATERIALS}

A. Clean, potable water

B. Portland cement

C. Hydrated lime

D. Sand

E. Epoxy cement

F. Teflon or nylon pins with scored and threaded surface. (Stainless steel or bronze may be used if teflon or nylon are unavailable).

\subsection{EQUIPMENT}

A. Stiff bristle brushes or vacuum

B. Trowels

C. Hawks

D. Carbon-tipped masonry bit

\section{PART 3---EXECUTION}

\subsection{ERECTION, INSTALLATION, APPLICATION}

A. Patching small cracks and holes with grout (a wetter version of mortar mix):

1. Cut out the deteriorated area to a sound surface. Under-cut the edge where possible to create a "key".

2. Brush or vacuum out all dirt or debris.

3. Flush the area with clean, clear water. Be sure no standing water remains.

4. Mix 1 part Portland cement, ASTM C150, Type I, part hydrated lime, and 2 to 3 parts sand -OR-

1 part Portland cement Type $\mathrm{P}$ (lime pre-mixed), and 2 to 3 parts sand NOTE: ADJUST MIX TO MATCH COLOR, TEXTURE AND PHYSICAL PROPERTIES OF THE ORIGINAL MORTAR.

5. Thin mortar to a slushy batter consistency.

6. Trowel apply mortar to damaged area in layers no more than 1 inch thick. Several layers may be required. DO NOT APPLY PATCHES OVER JOINTS.

B. Patching large chips, holes or broken corners using pins and mortar:

1. Using a stiff bristle brush, clean surfaces to be joined or patched.

2. Using a carbon-tipped masonry bit, drill staggered rows of holes approximately 2 inches deep (no more than 4 times the pin diameter), 1-1/2 inch apart and $1 / 8$ inch wider than the pin diameter.

3. Again, brush debris from the surface.

4. Fill the holes with mortar mix or epoxy cement.

5. Set the pins in the holes. 
6. Trowel apply mortar to damaged area in layers no more than 1 inch thick. Several layers may be required. DO NOT APPLY PATCHES OVER JOINTS.

7. To patch a chipped corner, fill the cavity with the concrete mix or bonding material, forcing it in and around the exposed pins, which will act to support and reinforce the patch.

C. To rejoin two broken parts (such as a concrete baluster), coat both broken surfaces with epoxy adhesive and gently tap the parts together. 


\section{Stage III - Lighting}

Historic light fixtures and the quality of light they produce, in combination with original daylighting features, contribute significantly to the character and authenticity of historic buildings. However, many historic buildings, unfortunately, have been compromised by inappropriate lighting alterations. ${ }^{27}$

Standard lighting was used throughout the cantonment during the construction of the WWII buildings like Bldg. 550 (see Figure 80). Bldg. 550 has most of its original light fixtures on the exterior and the interior as detailed in the plans from 1942. Below are figure photographs showing detail and examples along with treatment measures and information regarding lighting.

Figure 80. Plan Number 800-199, Standard Electrical Details, 1942.

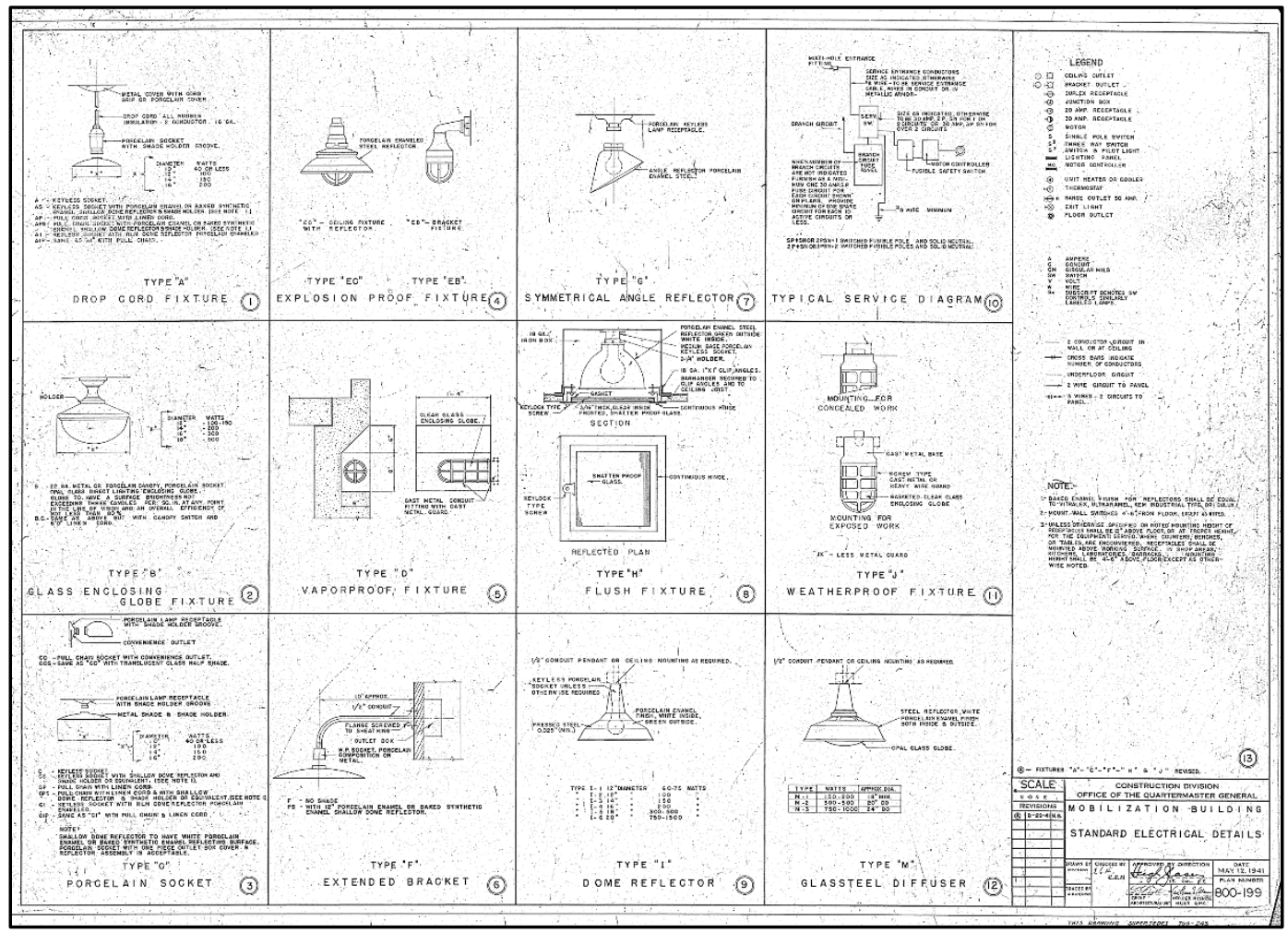

Source: Fort McCoy DPW.

27 https://www.gsa.gov/cdnstatic/TechnicalGuideLightingFINAL2.pdf. 


\subsection{Immediate concerns for lighting}

Successful lighting design for historic buildings considers a variety of factors for meeting multiple goals, including but not limited to:

- Preservation of historic materials and character,

- Occupant comfort,

- Functionality,

- Initial costs,

- Operational costs,

- Maintenance requirements,

- Disposal costs and environmental impact, and

- Aesthetics.

Consider rewiring historic fixtures as necessary to extend their lifespan.

\subsection{Exterior lighting}

As shown in Figure 81, the exterior light fixture is missing its bulb and shade, and there is a significant amount of rust present. Due to this fixture's condition, it needs to be replaced in-kind. In the original drawings, this type of fixture is not shown for exterior use, see Figure 82 for the typical exterior light fixture.

Figure 81. Looking at an exterior original base of light fixture with original light socket, missing the bulb and shade, located on west side of Bldg. 550, 2018.

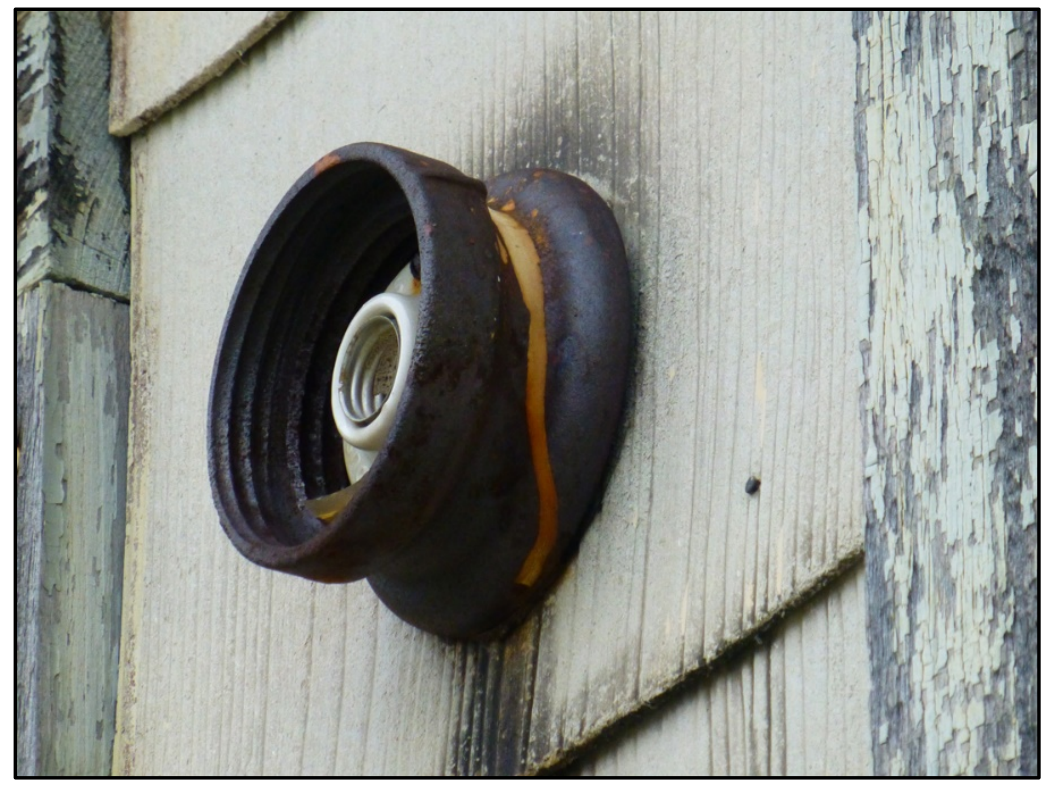


Figure 82. Detail in original plans of exterior light fixtures, 1942.

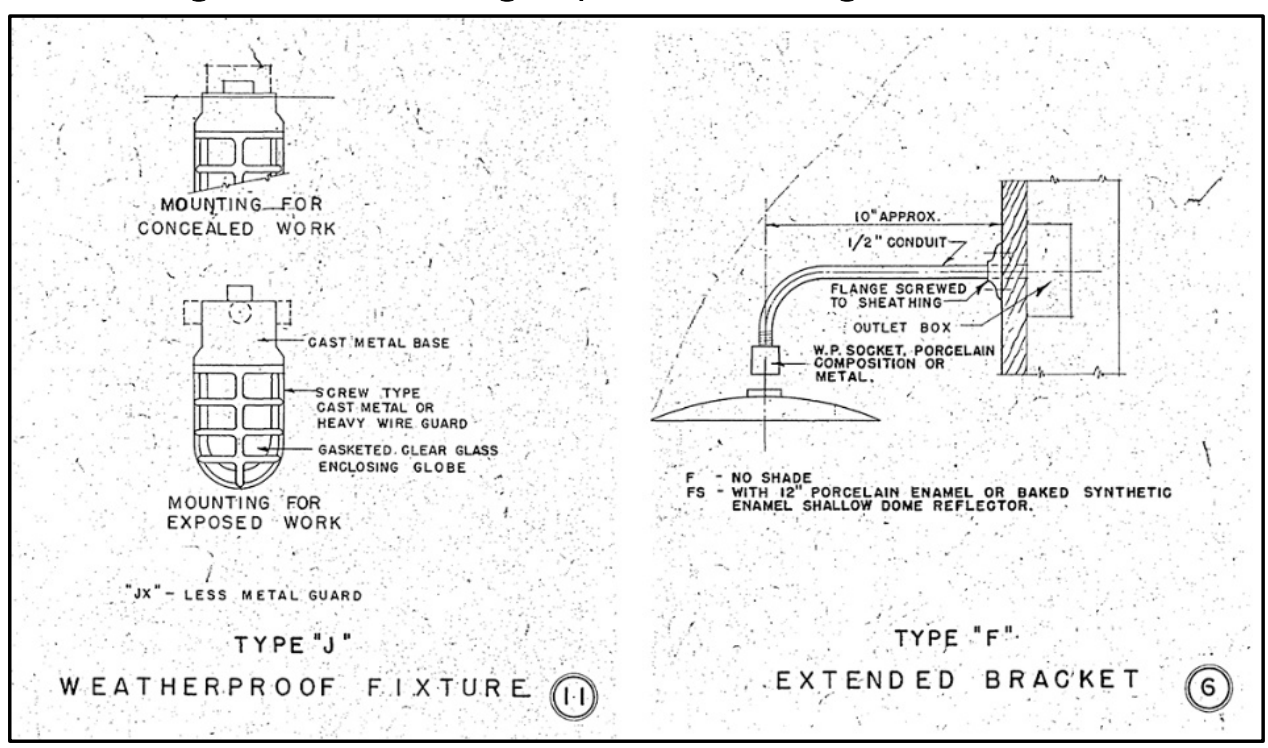

Source: Fort McCoy DPW.

The light fixture shown in Figure 83 is not original. Lights of this kind should be replaced with in-kind fixtures appropriate for the time of construction (see Figure 80) for examples of original fixtures.

Figure 83. Image of replacement exterior light fixture on north side over main entrance door, 2018.

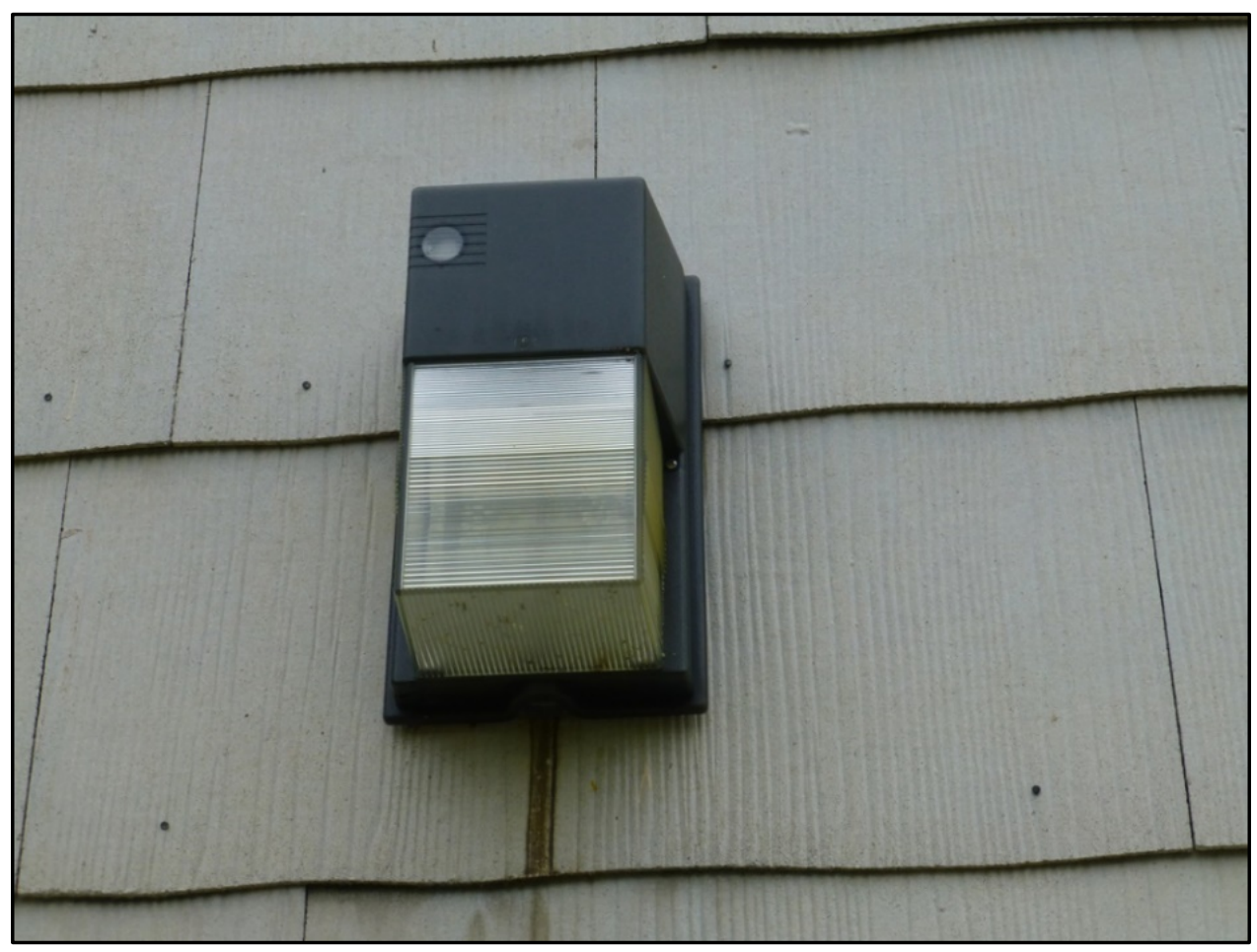




\subsection{Interior lighting}

There are two original light fixtures located in the toilet room above the sinks in Bldg. 550. The porcelain lamp receptacle and the translucent glass half-shade (see Figure 84), was a standard design pull-chain light fixture according to Plan Number 800-199 it was a "Type C" fixture, also detailed in (Figure 85). These two fixtures are in GOOD condition. It is rare for a building to have its original light fixtures from as early as 1942.

Figure 84. Image of original “Type C” porcelain socket, pull-chain lights in Bldg. 550, 2018.

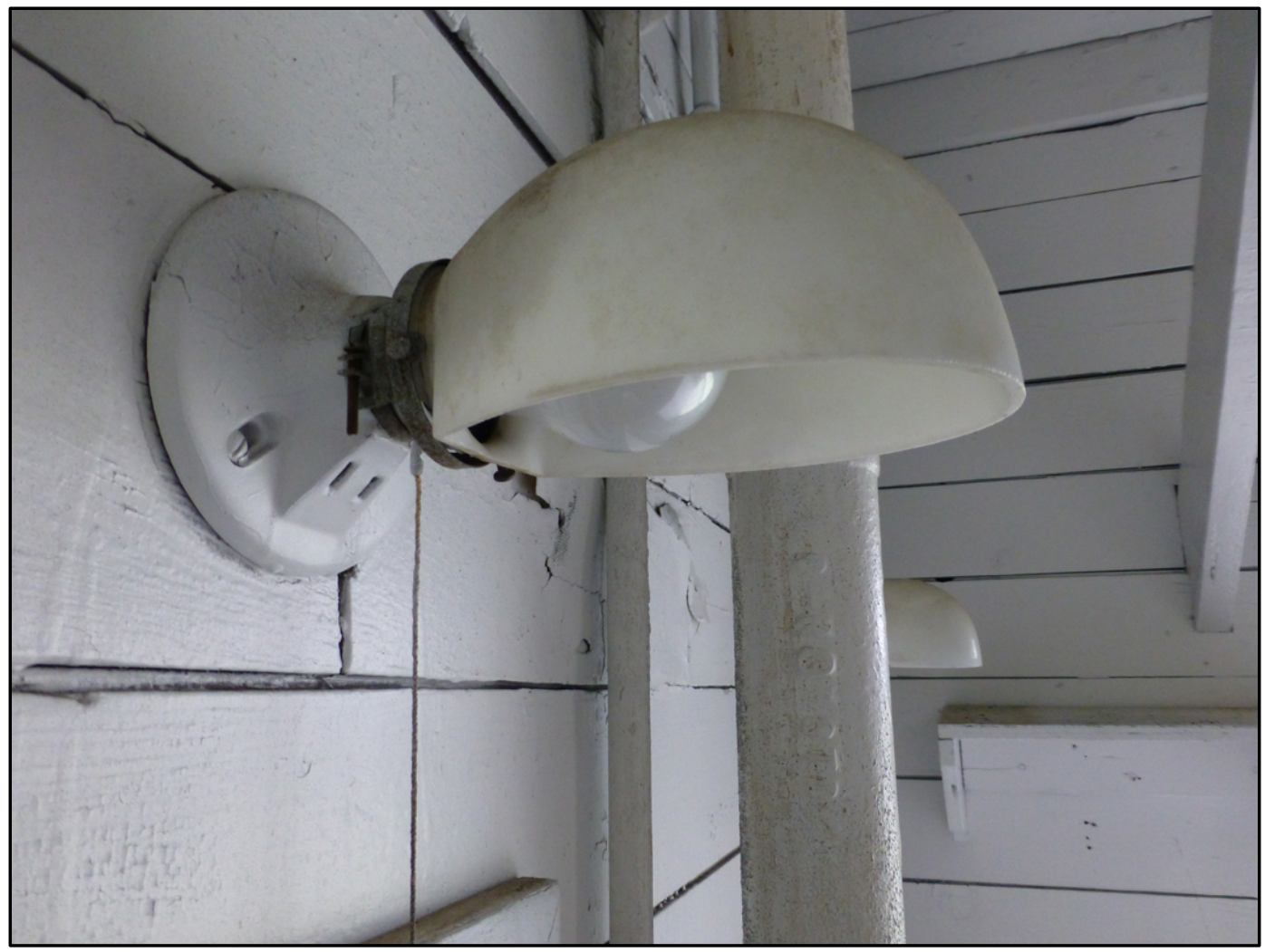

Figure 85. Detail from original Plan Number 800-199 of light fixture, 1942.

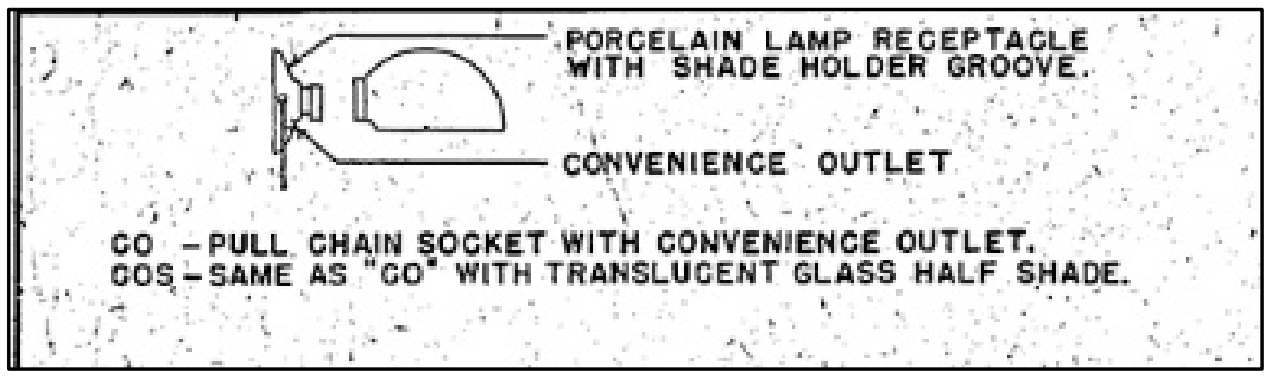

Source: Fort McCoy DPW. 
Another original light fixture in Bldg. 550 is located on the ceiling in a small room within the apparatus room. It includes a porcelain lamp receptacle and a metal shade and shade holder, as seen in Figure 87. In Figure 86 it is clear that there is rust on the inner metal shade. The pull-chain has been damaged and repaired.

Figure 86. Looking at an original ceiling light, 2018.

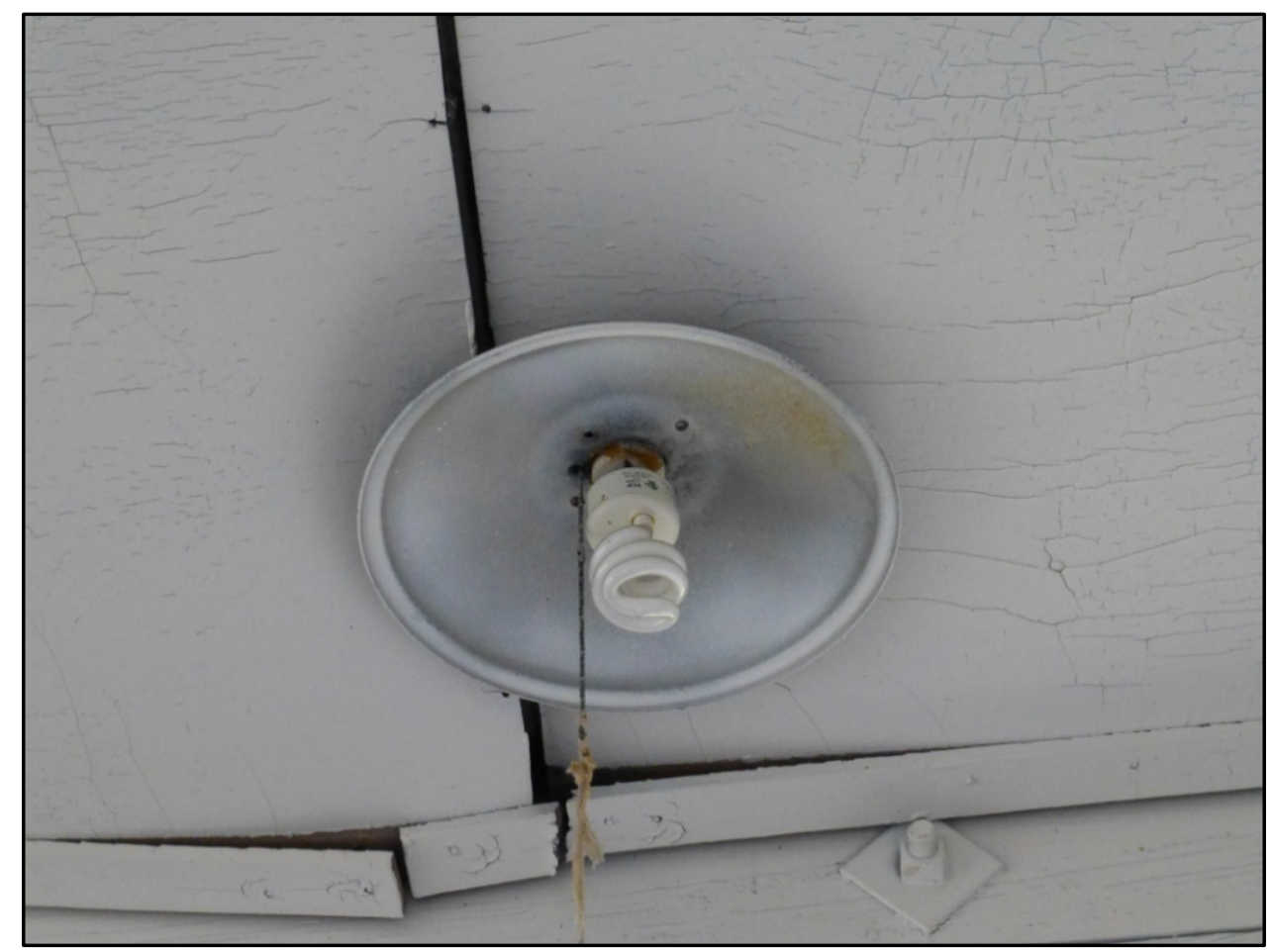


Figure 87. Detail from original Plan Number 800-199 of ceiling light fixture, 1942.

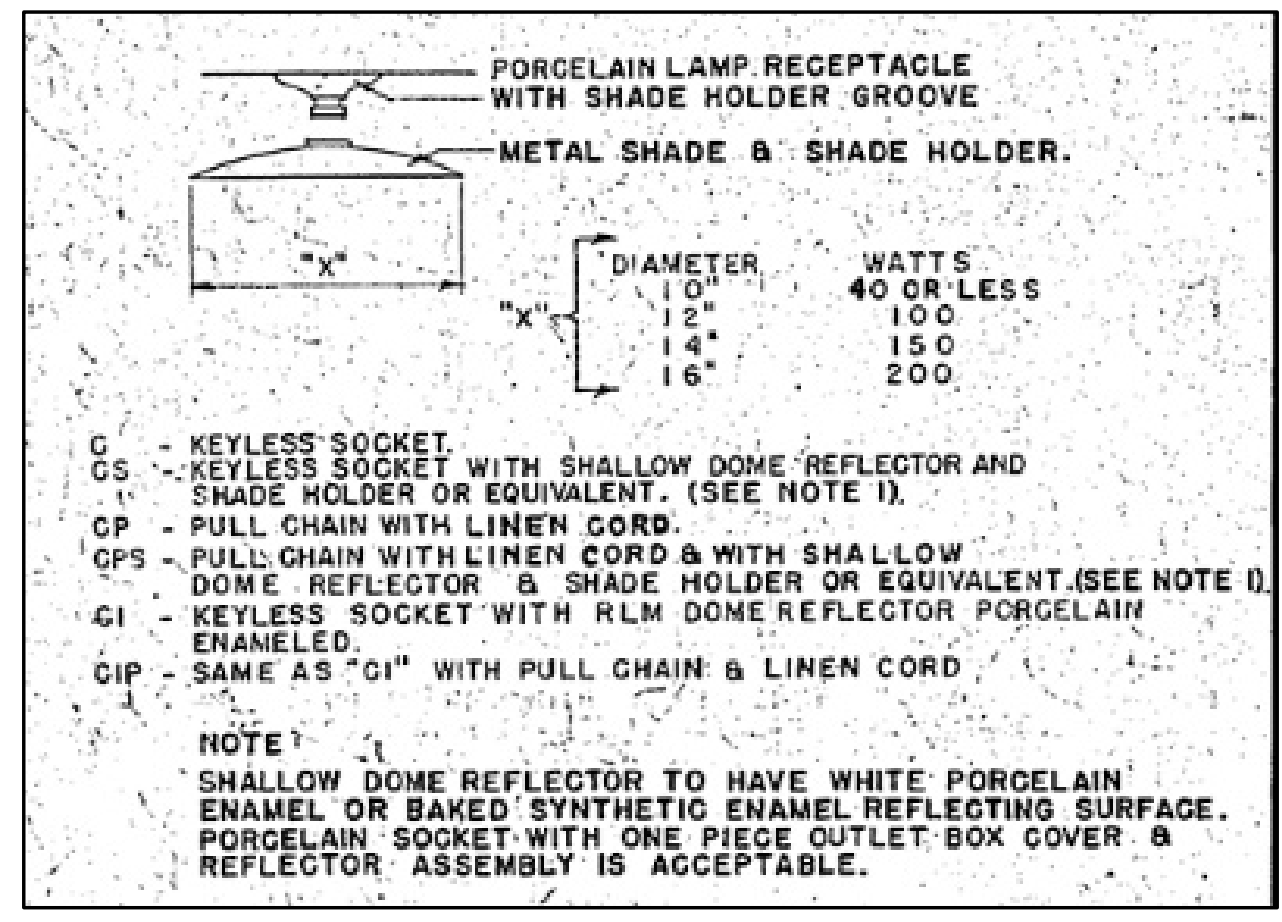

Source: Fort McCoy DPW.

\subsection{Treatment measures}

There is a mixture of original and replacement light fixtures in Bldg. 550. Below are documents that discuss upgrading historic lighting and sources to turn to when working with original light fixtures. All of these serve as appropriate preservation guidelines and conform to the Secretary of the Interior's standards for the treatment of historic buildings. 


\subsubsection{Technical Preservation Guidelines, 282009 (GSA.gov).}

\section{TECHNICAL PRESERVATIDN GUIDELINES}

\section{UPERADING HISTORIC BUILDING LIGHTING}

\section{CARDLINE ALDERSDN$$
\text { EXECUTIVE SLMMARY }
$$

Historic light fixtures and the quality of light they produce in combination with original daylighting features, contribute significantly to the character and authenticity of historic buildings. Many historic buildings, unfortunately, have been compromised by inappropriate lighting alterations. Electrical upgrades, space alterations and modernization projects often provide opportunities to reclaim compromised historic character while improving a historic building's marketability and value

This guide focuses on preservation-appropriate solutions for restoring, retrofitting and supplementing historic incandes cent lighting to reduce energy use and improve lighting functionality. Often the need to supplement historic fixtures can be eliminated by making the most of the building's original daylighting features and by appropriately retrofitting existing fixtures with new energy conserving lamps. Although this guide does not address in detail upgrading of modern-era fluorescent lighting, many of the solutions outlined here are applicable to buildings and fixtures of any era.

Additional guidance for meeting other lighting improvement goals is available from GSA's High Performance Gree Buildings program. All projects affecting historic building restoration zones require review by GSA's Regional Historic Preservation Officer (RHPO), beginning early in project planning to ensure that design scopes, qualifications, and budgets address preservation compliance requirements.

GSA Building Preservation Plans (BPPs) and Historic Struc ture Reports (HSRs) identify significant spaces and historic fixtures that may require restoration or special care retrofitting appropriately to meet current performance or energy conservation goals. BPPs and HSRs also identify locations where historic lighting has been removed that should be replicated as part of an overall lighting or space upgrade.

Historic lighting qualities to be considered in planning GSA historic buildings projects include

- Electric lighting thoughtfully supplementing daylight

- Variable light levels with electric lighting placed to support tasks and highlight architectural design
Lamps in a temperature range that casts "warm" light

Successful lighting design for GSA historic buildings consid ers a variety of factors for meeting multiple goals, including but not limited to:

- Preservation of historic materials and character,

- Occupant comfort

Energy conservation

Initial costs,

- Operational costs

- Maintenance requirements

- Disposal costs and environmental impact, and

- Aesthetics.

When historic drawings for restoring historic chandelier and other decorative fixtures or replicating historic fixtures are not available in GSA's BPP, HSR or the Public Buildings records of the National Archives and Records Administration, work with GSA's RHPO to identify similar buildings that may serve as a source for comparable examples to be used as a basis for restoration. Existing historic fixtures may be retrofitted with compact fluorescent lamps, reflectors, light emitting diode (LED) and other light sources to inc rease light output and energy efficiency. High efficiency incandescent lamps appropriate for use in historic light fixtures featuring exposed incandescent bulbs are currently in development and expected to be available in 2010 . When specifying replica fixtures, consult the GSA's RHPO and electrica engineering or sustainability experts to determine the most cost effective, preservation-appropriate lamp or lamp/ballast combination for meeting GSA performance, energy conservation and preservation goals in new period fixtures.

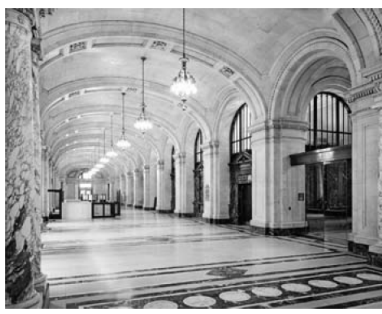

FIGURE 1 Electric lighting thoughtfully supplements daylight in this historic lobby.

\section{CENTER FUR HISTORIC BLILDINGS DFFICE DF THE CHIEF ARCHITEC PUBLIC BUILDINGS SERVILE GSA
U.5. GENERAL SERVICES ADMINISTRATION}

28 Retrieved from: https://www.gsa.gov/real-estate/historic-preservation/historic-preservation-policytools/preservation-tools-resources/technical-documents. 
Any new supplementary lighting necessary to meet GSA lighting requirements should be designed and placed inconspicuously to avoid detracting from the historic lighting and architecture. All lights can be placed on motion sensors to conserve energy when spaces are not occupied. Supplementary lights can be activated by daylight sensors, where appropriate, to meet space lighting needs when daylight is insufficient.

Indirect lighting tucked in coves or on cornices, combined with task lighting, can be a good alternative for boosting light levels in historically significant spaces. Simple sconces designed to blend into walls are another preservation-appropriate option for increasing ambient light levels without competing with historic ceiling lights. Recessed ceiling lights should be installed only as a last resort for supplementing historic ceiling lights and may be installed only in ceiling perimeters.

To ensure that GSA's preservation and performance expectations are met, specifications for historic lighting replication modification, or supplementation need to include requirements for product data, sample review and mock up test installation.

\section{INTRDDUETIDN}

Historic light fixtures and daylighting play a major part in a building's unique historic character. GSA's historic public buildings typically offer abundant windows and light courts admitting generous daylight to perimeter offices in doubleloaded corridor floor plans. Awnings, shades, and blinds provided light and glare control for exterior windows, with transoms, glazed doors, and glazed partitions admitting light into corridors and other interior spaces. Skylights provided daylight for spaces lacking windows

Ceremonial corridors, stairways and public spaces were often embellished with custom-fabricated pendant lights, chandeliers, or sconces hung from ornamental ceilings and walls. Desk lamps and torchieres supplemented ceiling lights in standard offices and courtrooms to put light where it was most needed.

When fluorescent lighting emerged as the principal approach for achieving uniformly high light levels, daylighting features such as glazed transoms and partitions were often removed or obscured. Skylights were commonly covered over as an emergency measure for public safety during World War II blackouts or during subsequent roof repairs to eliminate maintenance. At some historic buildings, original light fixtures have been replaced with inappropriate lighting that introduces glare from lamps rendering cool hues unsympathetic with the building's historic character

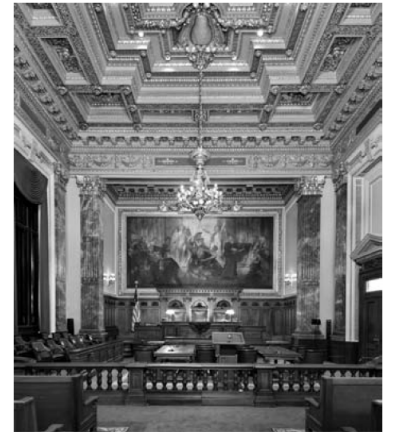

FIGURE 2 Variable light levels and multiple light sources contibute to the historic character and elegance of this early 20th century courtroom.

GSA's Office of Federal High Performance Green Buildings provides guidance on sustainable design, energy efficiency, resource conservation, and workspace quality for GSA repair and alteration projects of all scales. There are many ways that energy reduction and workspace needs can be met while preserving character-defining historic light fixtures and making the most of inherent energy conserving features in historic buildings. Lighting upgrades and interior renovation projects often provide opportunities to remove inappropriate alterations and restore compromised spaces to improve tenant satisfaction and make a historic building more marketable.

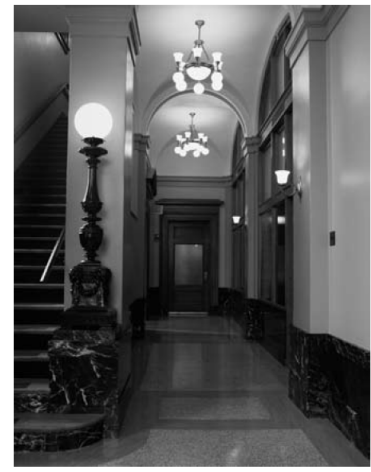

FIGURE 3 Interior upgrade projects often provide opportunitios to provide opportunities to proces and make a his spaces and make a historic building more marketable

Preservation scope of work requirements for lighting upgrades at historic buildings include, in order of priority

- Retaining and restoring historic light fixtures

- Optimizing historic daylighting features

- Retrofitting historic light fixtures appropriately with new lamps to meet current energy conservation and

CENTER FUR HISTORIC ELILDINGS

QFFICE OF THE CHIEF ARCHITECT PUBLIC BUILDINGS SERVICE U.5. GENERAL SERVICES ADMINISTRATIDN 
performance requirements

- Supplementing historic lighting appropriatelyonly when performance requirements cannot be met by daylighting and retrofitting solutions

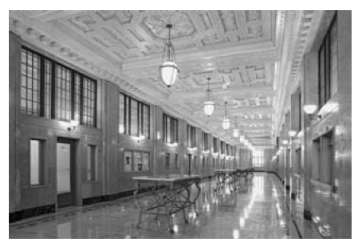

FIGURE 4 Good lighting depends on effective distribution of natural and electric light.

New guidelines recognize that good workspace lighting depends as much on effective light distribution as light output measured in foot candles and that excessive lighting can hamper productivity while consuming more energy than necessary. Uniform high-output "factory style" ambient lighting approaches formerly used to upgrade open area workspaces are no longer considered appropriate for most GSA properties. Variable lighting levels tailored to the specific tasks and design of a historic space can support preservation goals while improving workspace quality and comfort. Multiple lighting sources offer greater flexibility for addressing differing needs for ambient, task, safety, and accent lighting.

\section{FEDERAL GUIDES AND STANDARDS}

Every successful GSA historic building project begins with review of the specific building's Building Preservation Plan (BPP) or Historic Structure Report (HSR) to ensure that GSA teams involved in developing project requirements and overseeing design and execution are well informed on the building's preservation goals at the earliest stages of project planning and design. The BPP or HSR identifies spaces of architectural importance and character-defining features, such as historic light fixtures, to be preserved. BPPs and HSRs also outline restoration goals for altered public spaces, sometimes including detail drawings of original fixtures as a basis for replication.

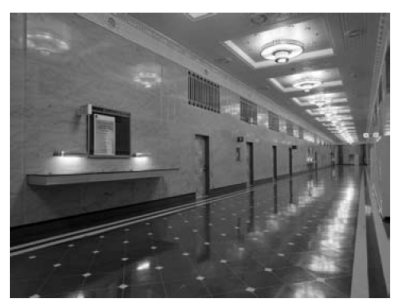

FIGURE 5 GSA Building Preservation Plans identify spaces of architectural importance and character and character-defining historic light fixtures, to be preserved.
Standards and guidance for all federal projects involving historic buildings are provided in the Secretary of the Interior's Standards for Rehabilitation and guidelines for applying the standards published by the National Park Service (NPS), U.S. Department of the Interior (DOI Standards). The National Park Service has also published a variety of briefs and technical guidance addressing a range of lighting improvement issues. All guidance publications are available free online at www.nps.gov/hps/tps/publications. htm.

Central to the DOIStandards, which are based on European standards used throughout the world, are the principal goals of:

- maintaining authenticity, by

doing no harm to historic materials,

- designing changes sympathetically, and

- restoring significant spaces correctly, based on historic documentation.

GSA's PBS P100 Facility Standards provide guidance for lighting improvements at GSA historic properties, as follows:

PBS P100 Federal Standards for the Public Buildings Service

Chapter 6. Electrical Engineering, Lighting - Historic Structures

Historic fixtures may be upgraded with energy efficient lamps, ballasts, reflectors, or other means to achieve required light levels, if changes can be made without affecting the appearance of the fixture. Energy efficient light sources should match the warm to white color range of incandescent light or daylight as closely as possible. In restoration zones, opportunities should be sought to replace unsympathetic contemporary lighting with replicas of original historic fixtures. Replica fixtures in which light sources are not exposed should incorporate high output, energy efficient lamps as necessary to achieve required light levels and meet energy conservation standards. Supplemental lighting, if required, should be designed and installed to avoid competing visually with historic lighting. Freestanding torchieres, task lighting and discrete accent lighting are recommended for increasing light levels in ceremonial spaces containing ornamental ceilings and historic chandeliers.

CENTER F DR HISTORIC BUILDINGS OFFICE DF THE CHIEF ARCHITECT PUELIC BUILDINGS SERVICE ப.5. GENERAL SERVICES ADMINISTRATION 


\section{RETRDFITTING HISTDRIC LIGHTS}

The simplest and least expensive method of reducing ener gy use in spaces containing historic light fixtures with incandescent lamps concealed by translucent or opaque globes, shades, or lenses, is to replace the incandescent lamps with compact fluorescent lamps. Changing to a fluorescent source with color temperature as close as possible to that of the incandescent lighting ( $2700 \mathrm{Kelvin}$ ) will help to ensure against lighting color changes that may have a negative impact on the appearance of historic finishes and features.

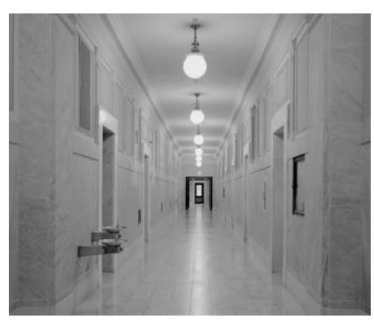

FIGURE 6 Replacing incandescent lamps incandescent lamps with compact fluorescent lamps is one of the simplest ways to reduce energy use in spaces containing historic light fixtures. Installing energy efficient ballasts and lamps ballasts and lamps provides even greater long-term operational
cost savings.

Recent advances have increased the range of preservationappropriate retrofitting options, with initial cost generally proportionate to operational savings. Select lamps providing color temperatures as close as possible to that of origina lighting (2700 Kelvin for standard incandescent lights). Lighting consultants specializing in historic lighting will be best qualified to analyze existing historic lighting (foot candles and temperature) and identify appropriate alternatives.

Where slow intitial lamp brightness (five to six minutes) is no a concern, screw-in metal halide lamps in the 3000 Kelvin range with lifetimes up to 12,000 hours are now available as another option for replacing incandescent lamps concealed by translucent globes or housings. Screw-in lamps containing LEDs that will fit many historic fixtures are also available in a 2700 Kelvin option, offering lifetimes up to 50,000 hours (compared to $750-1500$ hours for incandescent lamps), where the fixture configuration and room ventilation will prevent excessive heat build up. Where feasible, this option of fers the greatest potential for operational savings, albeit at the highest initial cost, making its use most advantageous for difficult to reach locations and for fixtures that must be partially disassembled for lamp replacement.

Programmed start ballasts, occupancy sensors, bilevel ballasts, daylight sensors and addressable controls offer additional operational savings that should be explored as part of a comprehensive lighting upgrade. Care should be exercised in the selection of the appropriate lamp in fixtures that may be part of the building emergency egress lighting system. Fixtures that are connected to the buildig emergency geerator must be able to come to full brightness very quickly

Compact fluorescent, metal halide, and screw in LED lamps are not appropriate for historic chandeliers featuring exposed incandescent lamps. However, High Efficiency Incandescent (HEI) lamps that use $25 \%$ of the energy standard incandescent bulbs use will offer an energy saving alternative for these fixtures as early as 2010 .

Historic fixtures that do not provide adequate lighting may sometimes be modified to include additional light sources, such as light emitting diodes (LEDs) or fiber optics, where modifications can be done inconspicuously and lamp temperatures (color) will be compatible.

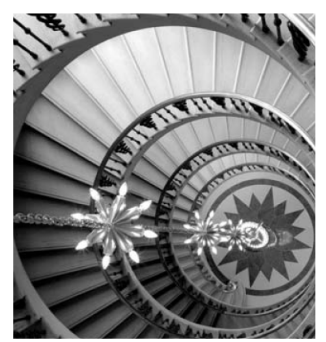

FIGURE 7 Installing highefficiency incandescent (HEI) lamps in historic exposed builb fixtures reduces energy use by $50-75$ percent with no impact on the appearance of the fixture.

In historic spaces requiring increased light levels, apply the following order of preference:

1. Retrofit historic lights

2. Supplement historic lights with discretely placed preferably indirect, lighting to avoid competing with historic lighting.

\section{REPLICATING HISTDRIC LIGHTS}

Historic lighting replication projects often create opportunities to improve lighting performance and energy efficiency in a visually authentic reproduction fixture. When specifying replica fixtures, consult the GSA's RHPO and electrical engineering or sustainability experts to determine the most cost effective, preservation-appropriate lamp or lamp/ballast combination for meeting GSA performance, energy conservation and preservation goals.

Most period lights can accommodate retrofit for ballasts for compact fluorescent lamps and some will accommodate a substantial reconfiguration of concealed additional light-

CENTER F OR HISTORIC BUILDINGS DFFICE OF THE CHIEF ARCHITECT PUELIC BDILDINGS SERVICE U.5. GENERAL SERVICES ADMINISTRATIUN 
ing sources for improved light output. Reproduction historic lights for significant spaces such as courtrooms may be fitted with a combination of light sources and separate switched or dimmable settings to allow for multiple light levels such as a working light level and historic light level for ceremonia events.

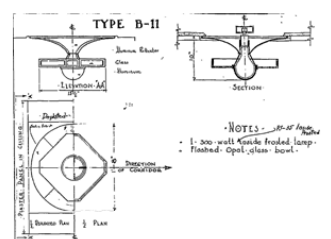

FIGURES 8-10 Replica historic ceiling light incorporating a lengthened pendant and $\mathrm{CF}$ lamps to provide side lights in addition to the historic fixture's downlight, originally provided by a single incandescent bulb.
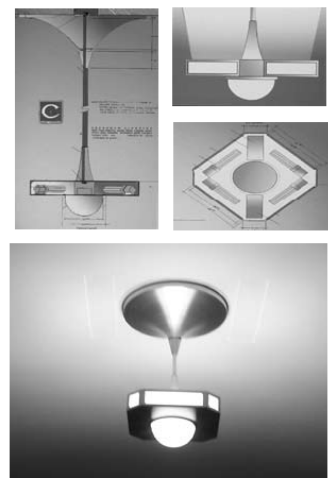

INTEGRATING DAYLIGHT

Recent research increasingly links daylight with productivity and health. Poor daylighting can lead to uncomfortable work environments, low employee satisfaction, poor productivity, and untenable buildings. Effective daylighting reduces the energy required to light and heat or cool a building. Most historic office buildings, fortunately, were designed with generous windows and a variety of features to convey and manage daylight through surface transparency, diffusion, and daylight control.

Historic daylighting design elements include:

- Windows and window shading devices

- Skylights and laylights

- Glazed doors, transoms, and sidelights

- Glazed and low partitions

- Transparent, translucent and reflective surfaces
Appropriately designed and installed window treatments (shades, blinds) and treated glazing control heat gain and glare while maintaining window views and admitting daylight. Some shading systems use photosensor-driven operating devices to increase or reduce daylight based on outdoor conditions. Energy conserving glazing films change thermal conductivity to control heat gain. Low-e films, as they are known, come in a variety of types that range in visual impact from negligible to inappropriately reflective for use on historic buildings.

Since the amount of light penetrating a room is limited by the height of the opening, it is important that windows are not partially blocked by suspended ceilings, wall partitions and other alterations. Design suspended ceilings to be recessed from window openings by creating pockets or soffits around windows or configuring ceilings to slope upward toward the head of the window or step down toward the corridor to accommodate ductwork and other building systems.

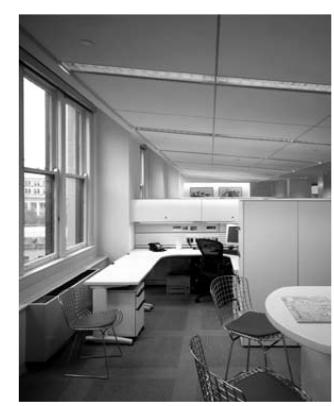

FIGURE 11 Preserve window opening clearance by recessing suspended ceilings from windows or configuring ceilings to slope upward toward the window head or step doun toward the building interior.

Low or glazed partitions allow light to penetrate into open office workspaces for building occupants not seated along exterior walls. Studies show that employees will generally choose a space offering generous daylight over a space offering privacy with no daylight, underscoring the importance of workspace design features that allow light to penetrate as far as possible into the building floorplate.

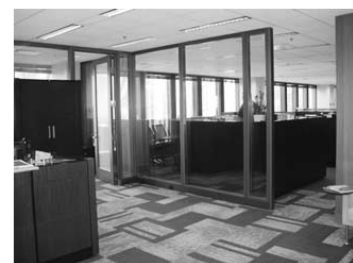

FIGURE 12 Low partitions and glazed partitions allow light to penetrate into workspaces.

CENTER FOR HISTORIC BUILDINGS DFFICE QF THE CHIEF ARCHITECT PUBLIC BUILDINGS SERVICE J.S. GENERAL SERVICES ADMINISTRATION 
Glazed doors, transoms, and interior windows "borrow" or capture daylight from exterior windows to provide natural lighting in corridors and interior spaces that otherwise would have little or no daylight. It is important to maintain the transparency (or translucency) of these features to gain these in tended daylighting benefits.

Many historic buildings include skylights designed to adm daylight to interior cores, underground spaces and other windowless areas. Skylight systems typically include interior laylights that may include a daylight control mechanism. Skylight repair and replication projects provide opportunitie to improve the thermal performance and life-cycle of these assemblies while improving the marketability of windowles workspaces

Reflective finishes such as polished stone walls and terrazzo floors can contribute significantly to lighting levels in corridors and other public spaces and also to the perception that these spaces are adequately lit. Accordingly, installa tion of carpeting or light absorbing materials outside of office areas where they are necessary for workspace noise reduction should be discouraged in favor of appropriate soundmasking devices.

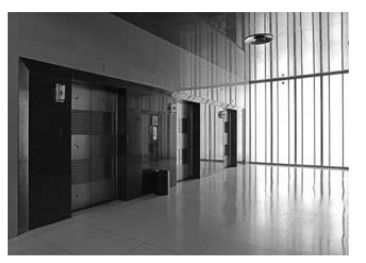

FIGURE 13 Reflective surfaces contribute significantly to lighting levels and quality in corridors and other public spaces.

All lights can be placed on motion sensors to conserve energy when spaces are not occupied. Supplementary lights can be activated by daylight sensors, where appropriate, to meet space lighting needs when daylight is insufficient.

For additional guidance on integrating daylight design, electrical illumination, architectural design and mechanical systems, refer to P100 6.8 Interior Lighting, Daylighting, and Control Systems. See also NPS Preservation Note 44 Natural Light in Historic Buildings.

\section{SUPPLEMENTAL LIGHTING}

Where historic fixtures cannot accommodate additional ligh sources or modification of existing lights will not achieve necessary light levels, existing light may be supplemented by contemporary lighting carefully designed and placed to maintain the visual predominance of the space's historic lighting.

First consideration for supplementing historic lights should be given to traditional task lighting solutions such as desk lamps that direct light onto the work surface where it is needed to perform tasks. Most open space furniture systems feature task lighting integrated into workspace shelving, significantly reducing ambient lighting needs. Occupants should be encouraged to use and maintain task lighting. In combination with photo-sensors and manual controls that reduce demand for ambient lighting in large work areas, task lighting gives occupants a means of controlling light levels within their own workspace, often netting substantial savings, as workers tend to prefer less ambient light than is typically installed in contemporary offices.

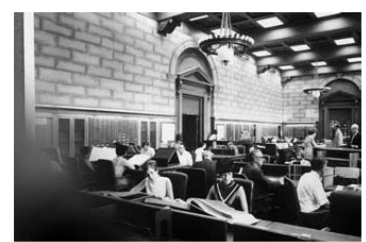

FIGURES 14-16 Traditional supplemental Fus (lition lamps preserve the architectural dominance of historic chandeliers and place light where it is needed to perform work. Top (before restoration): Surface mounted fluorescent lighting compromises the reading promises the reading room's coffered ceiling and competes with the chandeliers. Middle and Bottom (after restoration): Reader-controlled desk lamps eliminate the need for high ambient light levels, enabling the ceiling to be restored to its original appearance

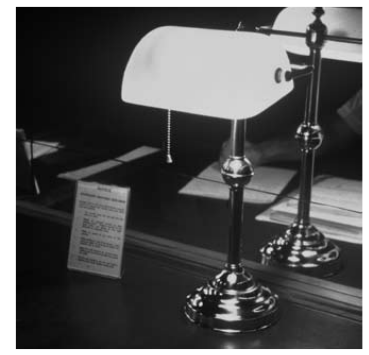

When historic fixture modifications and task lighting cannot adequately address ambient lighting needs, explore additional light-supplementing options that will not compete with historic lights. In historically significant spaces, supplementing chandeliers, pendant lights or other ceiling mounted lights with wall mounted sconces, uplights mounted on fur-

CENTER FOR HISTORIC BUILDINGS DFFICE QF THE CHIEF ARCHITECT PUBLIC BUILDINGS SERVICE U.S. GENERAL SERVICES ADMINISTRATION 
niture, or freestanding lamps is preferable to installing additional ceiling fixtures.

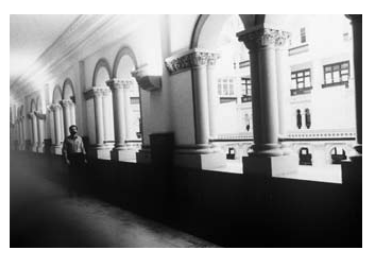

FIGURE 17 Contemporary sconce lights illuminate walls and ceilings in corridors where daylight originally served as the principal light source for this 19th-century post office. The opaque shades of the sconces reflect light against the walls of the corridor rather than beaming light down on corridor occupants, subtly maintaining the visual dominance of the skylit atrium the corridors overlook.

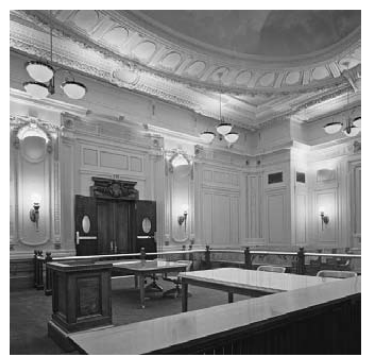

FIGURE 18 Quietly designed sconces maintain visual focus on the historic chandeliers and decorative finishes embellishing this historic courtroom

Contemporary wall sconces or staggered channel florescent or cold cathode tube lighting concealed in coves or on cor nices may be used illuminate corridor walls and ceilings with reflected light that maintains historic character and does not compete with historic ceiling lights or character-defining day lighting features.

Installing recessed or other ceiling lights in significant spaces containing original or reproduction ceiling lights should be considered as a last resort only for achieving light levels required to make a space functional. Recessed down lights may only be installed outside of the central ceiling field, along the perimeter or other edge locations. Care should be taken to avoid high output, direct lighting sources that may overpower or distract from ornamental historic lighting, reducing it to an architecturally and functionally ancillary role.

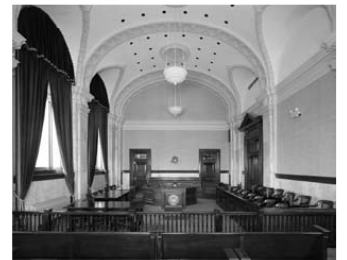

FIGURES 19-20

Fig. 16. Limit recessed lighting to the ceiling perimeter to avoid overpowering historic chandeliers.

Top: The punctures created by the installation of recessed lighting in this plaster ceiling introduce a

distracting contemporary grid and compete with the historic chandeliers, parhistoric chandeliers, par-

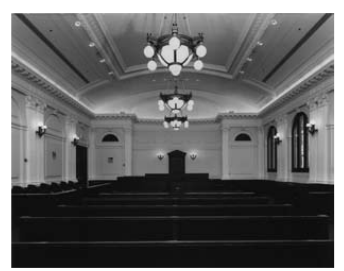

the new fix-

tures are It. Bottom: Plac-

ing recessed lighting away from the center, against the historic molding, minimizes competition with the historic chandeliers, the intended ornamental focus of the courtroom.

\section{SPECIAL ISSUES}

Modern-era buildings often contain historic fluorescent fixtures that need to be replaced or relamped for energy conservation or improved performance. Lighting specialists and architects specializing in buildings of this era are more equipped to assess the significance and adaptability of original lighting fixtures and the availability of appropriate replacement lighting than generalists unfamiliar with the unique attributes and requirements of modernist buildings. Modern-era light lenses, for example, may be significant components of the fixtures, but degraded, requiring analysis of conservation and component replacement options.

Exterior facade lighting and interpretive lighting to illuminate artwork or exhibits require specialized lighting design skills to identify options satisfying the interpretive, conservation, and maintenance needs of materials being illuminated. Any specialists retained to plan, design, or install exterior or interior interpretive lighting at historic properties must demonstrate an understanding and support of federal preservation standards and GSA preservation policy.

\section{MAINTENANCE PLANNINE}

Maintenance planning as part of design contributes to lighting solutions that perform well over the long term. Recommended steps for the continued success of a historic building lighting upgrade include:

1. Confirming the convenient availability of replacement lamps for new and upgraded fixtures before committing to purchasing or modifying any fixture;

CENTER FOR HISTORIC BUILDINGS QFFICE DF THE CHIEF ARCHITECT PUBLIC BUILDINGS SERVICE U.S. GENERAL SERVICES ADMINISTRATION 
- Maintaining a stock of replacement lamps;

- Ensuring that new and historic fixtures have similar temperature lamps to avoid mixing "warm" and "cool" hues in the same space; and

- Relamping fixtures with a space as a group to avoid uneven temperature (light color) distribution, as lamps change temperature over time.

\section{CDNCLபSIDN}

For all lighting modifications within historic building restoration zones, be sure to specify sample review of any new or modified lighting to be installed and require a mock up installation for RHPO and tenant review and approval prior to overall fabrication and installation, to ensure that preservation design criteria and the project's performance and esthetic requirements have been met. Budgeting time and funding for mock up installation serves as insurance against the possibility of cost increases and delays if a planned solution falls short of GSA and tenant expectations. Allow time and funds to make appropriate lighting adjustments.

Help GSA promote imaginative solutions that preserve historic lights and make the most of original daylighting and architectural features that make GSA's historic buildings unique and valuable. To share images and information documenting your own project successes for the benefit of future updates to this guide, contact caroline.alderson@gsa.gov.

For additional guidance and building specific inhistoricpreservation>Contacts for a current listing). 


\title{
8.4.2 Sources of historic lighting reproductions, ${ }^{29} 2017$ (GSA.gov).
}

\section{GSA U.S. General Services Administration \\ Sources of Historic Lighting Reproductions}

\author{
Procedure code: \\ $1650001 R$ \\ Source: \\ Technology \& Conservation, Summer/Fall 1992, pp. 22-24 \\ Division: \\ Electrical \\ Section: \\ Lighting \\ Last Modified: \\ 01/04/2017
}

This reference list includes manufacturers and suppliers of historic lighting reproductions as identified in the Summer/Fall 1992 issue of Technology \& Conservation.

- A.K. Exteriors (outdoor wall lanterns)

- American Lantern Company (chandeliers, outdoor wall lanterns, post lights (Colonial Williamsburg style))

- Appleton Electric Company

- Classic Illumination, Inc. (ceiling mounted units (1900-present), chandeliers, wall sconces (1870-present))

- Edison Price Lighting (ceiling mounted units)

- Gates Moore Lighting (chandeliers, outdoor wall lanterns, post lights)

- Gibson and Gibson Antique Lighting (ceiling mounted units, floor lamps, wall sconces (1880s-1940s styles))

- Herwig Lighting (outdoor wall lanterns, post lights)

- Historical Arts \& Casting, Inc. (chandeliers)

- King's Chandelier Co. (chandeliers, wall sconces (18th and 19th century))

- Lighting by Hammerworks

- Lite Makers, Inc.

- Luxo Lamps Corporation

- Metroplitan Lighting Fixture Co., Inc.

- Newstamp Lighting

- Progress Lighting

- Rambusch Lighting

- Saint Louis Antique Lighting Co.

29 Retrieved from: https://www.gsa.gov/real-estate/historic-preservation/historic-preservation-policytools/preservation-tools-resources/technical-documents. 
- Spring City Electrical Mfg. Co.

- Sternberg Vintage Lighting

- Sterner Lighting Systems Inc. (chandeliers, outdoor wall lanterns, post lights)

- Tower Lighting (chandeliers, outdoor wall lanterns, post lights (Colonial style))

- Versailles Lighting Inc. (ceiling mounted units, chandeliers, wall sconces)

- Victorian Lighting Works, Inc. (chandeliers, wall sconces) 


\section{Stage III - Plumbing Fixtures}

It is rare for a building of this age to still have toilet room fixtures intact, such as toilets, sinks, and shower stalls. Due to the age of these fixtures and their materials, there is minimal information regarding it.

\subsection{Enameled sinks}

There are two original enameled sinks located in the toilet room in Bldg. 550 (see Figure 88). The faucets are original. It is unclear if these are still functioning. They are in FAIR condition with some rust staining.

Figure 88. Two original porcelain enameled sinks in the toilet room in Bldg. 550, 2018.

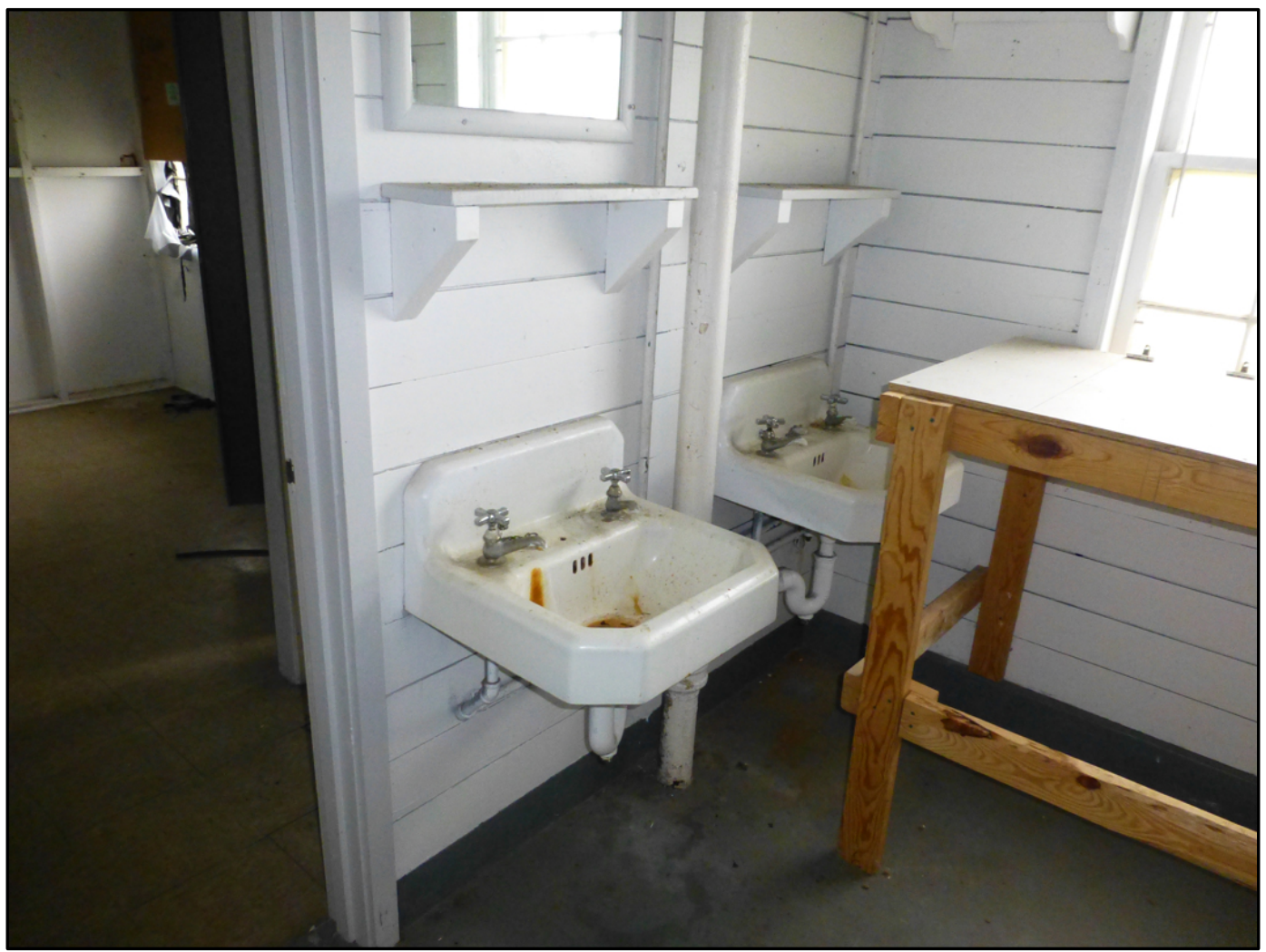

An original enameled slop sink is located in the former work room within the apparatus room in Bldg. 550 (see Figure 89). It is in POOR condition with exposed plumbing and major discoloration and stains. 
Figure 89. Looking at enameled slop sink in Bldg. 550, 2018.

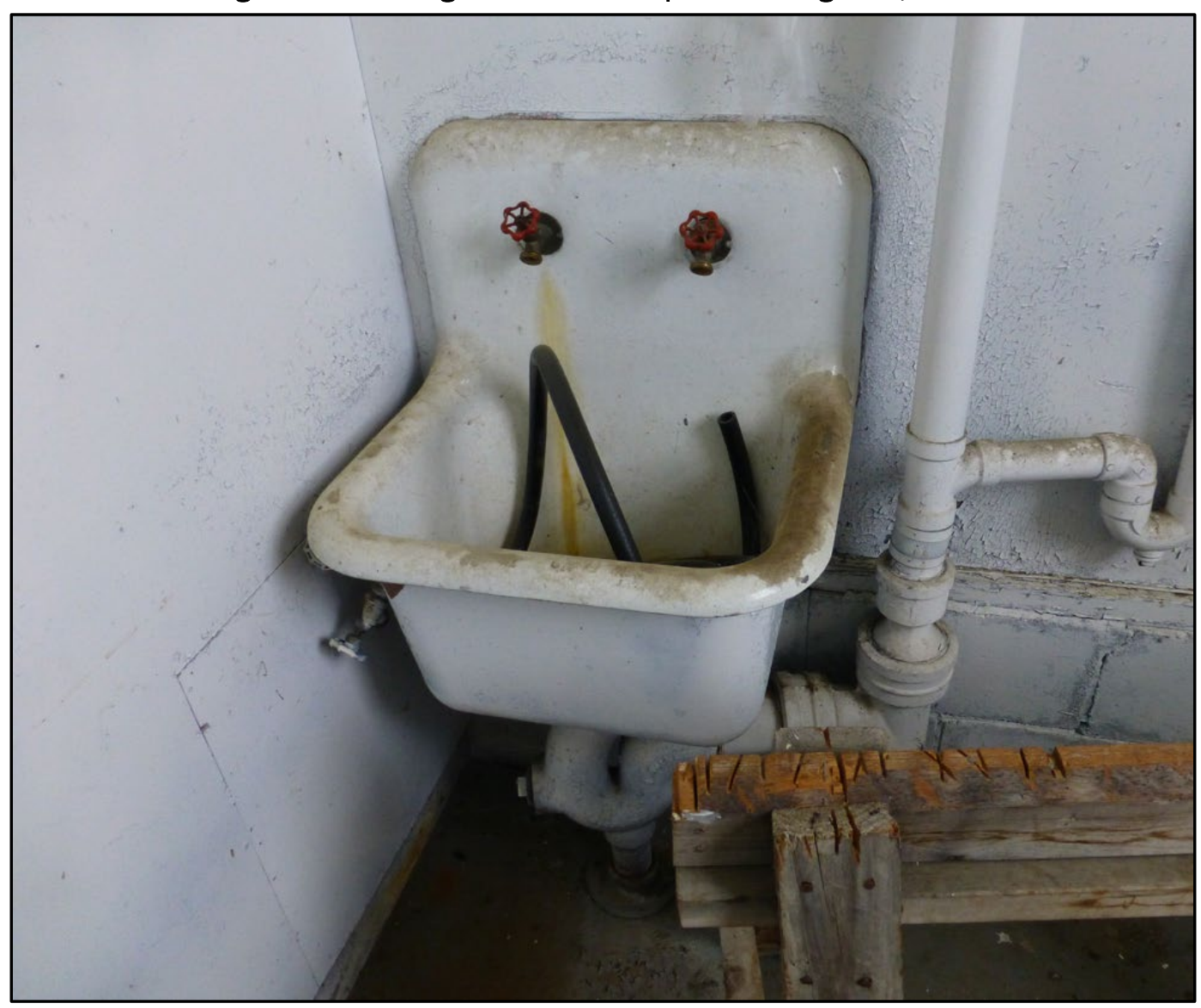

This 2018 photo shown in (Figure 90) shows an original enameled sink in the alarm room that is in POOR condition. There is major discoloration and stains, there appears to be pealing, scratches, and nicks, and the faucets are missing. As mentioned in footnote 5 , the original plans for the alarm room do not show an enamel sink. The enamel sink in the alarm room is of the period of significance and is treated as original to the building. 
Figure 90. Looking at enameled sink in Bldg. 550, 2018.

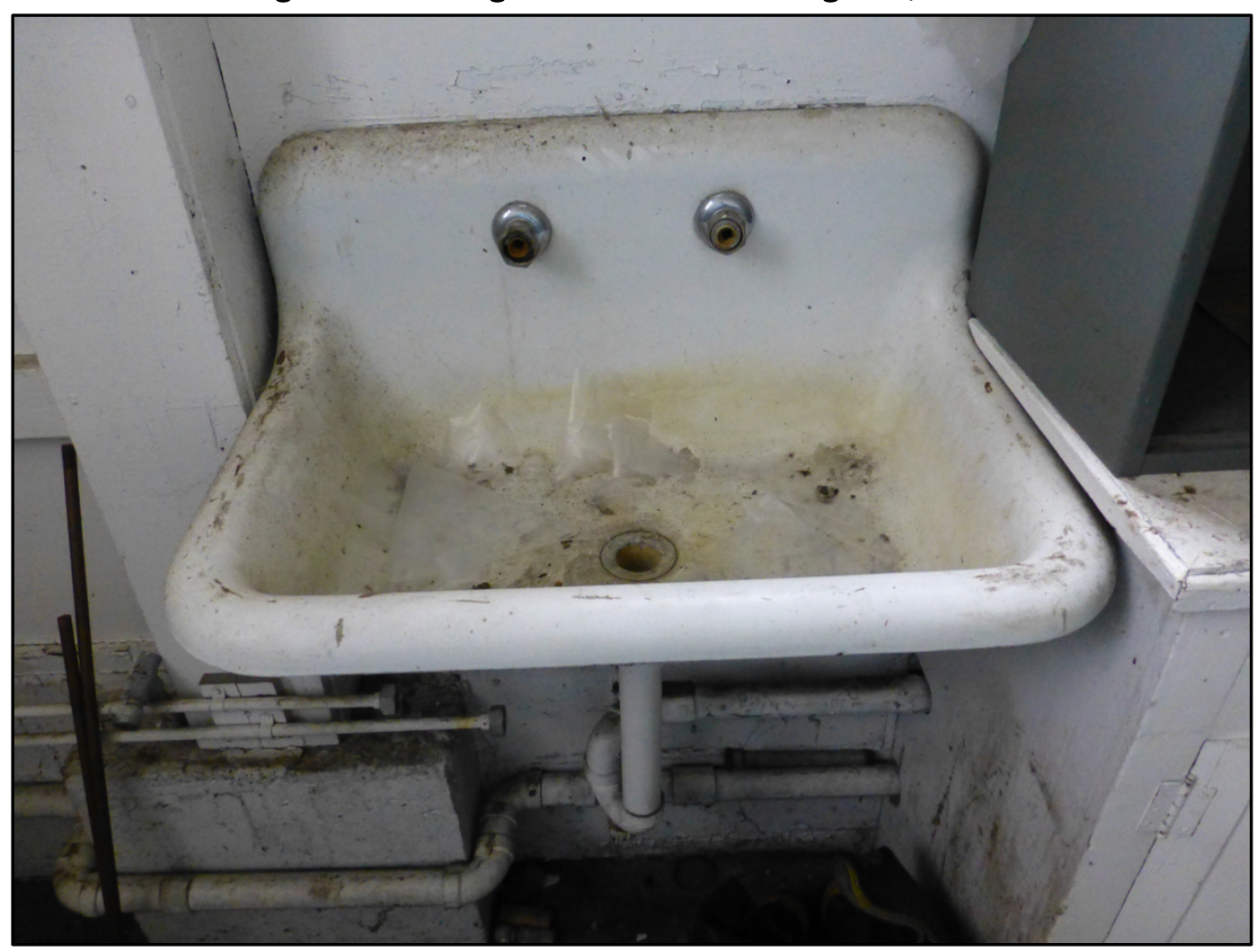

\subsection{Zinc sink}

There is a zinc sink located in the alarm room in Bldg. 550 (Figure 91). The faucets are not extant, and it is unclear if it is connected to the plumbing system. It is in GOOD condition. As mentioned in footnote 5, the original plans for the alarm room do not show a zinc sink. The zinc sink in the alarm room is of the period of significance and is treated as original to the building. 
Figure 91. Zinc sink in the alarm room in Bldg. 550, 2018.

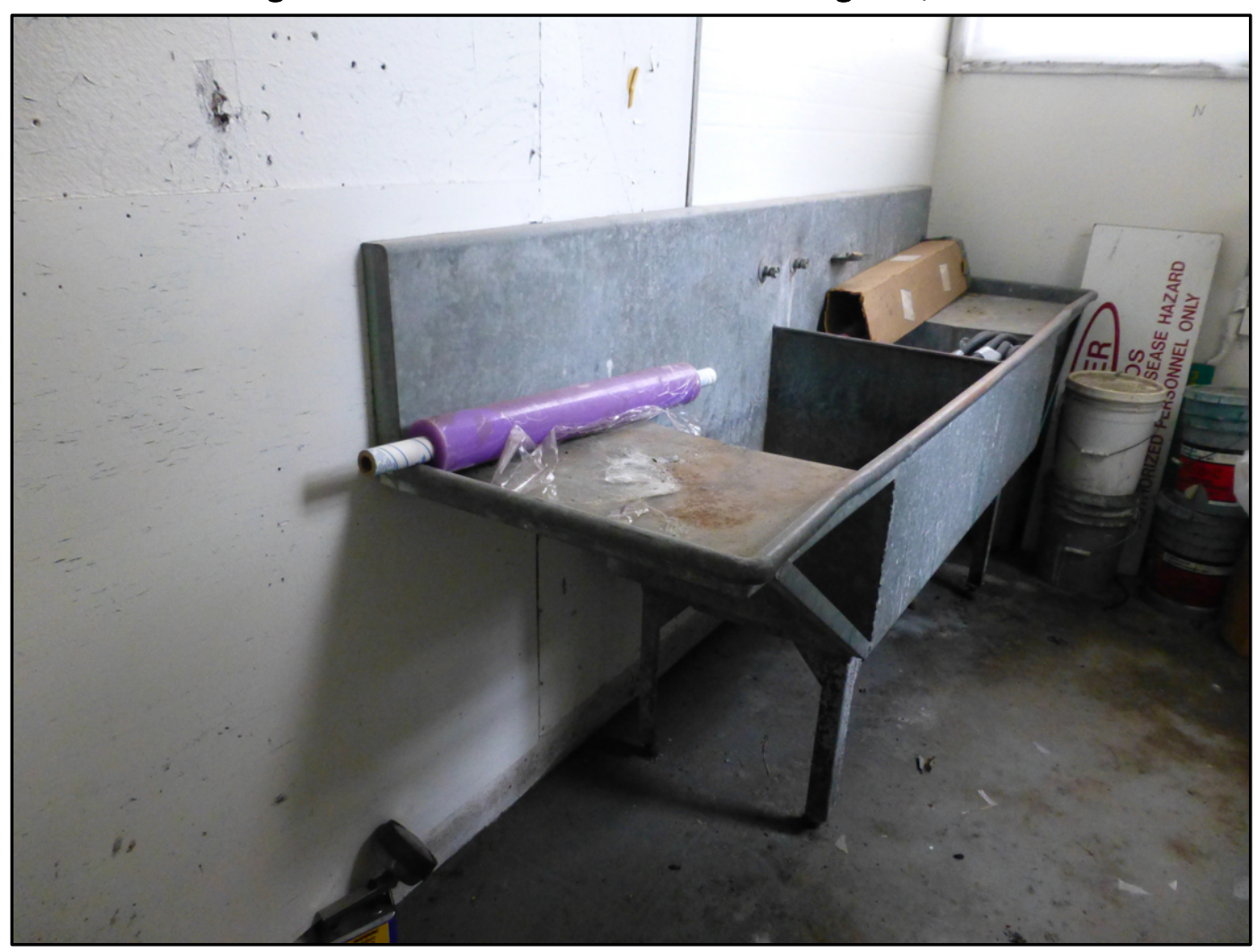

\subsection{Porcelain toilets and urinals}

There are two original porcelain toilets in Bldg. 550 (see Figure 92) for an example. This toilet itself appears to be in FAIR condition; however, there is discoloration. Both toilets are missing plumbing connections to the water supply and the original seat has been replaced. 
Figure 92. Looking at porcelain toilet in Bldg. 550 (toilet seat is not original), 2018.

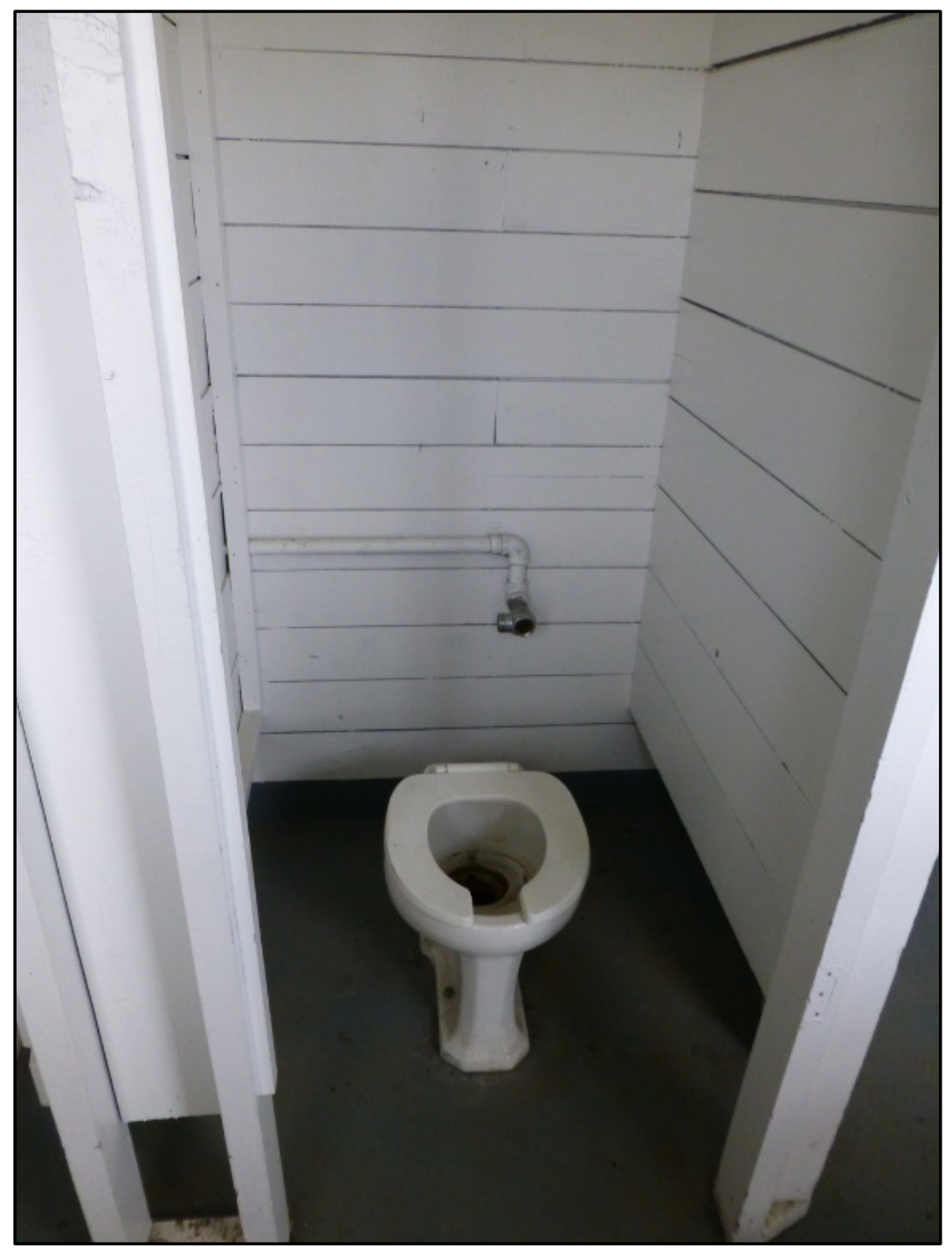

There is one original porcelain enameled urinal located in the toilet room in Bldg. 550 (see Figure 93). The urinal itself appears to be in FAIR condition; however, there is major discoloration. 
Figure 93. Original porcelain enameled urinal in the toilet room in Bldg. 550, 2018.

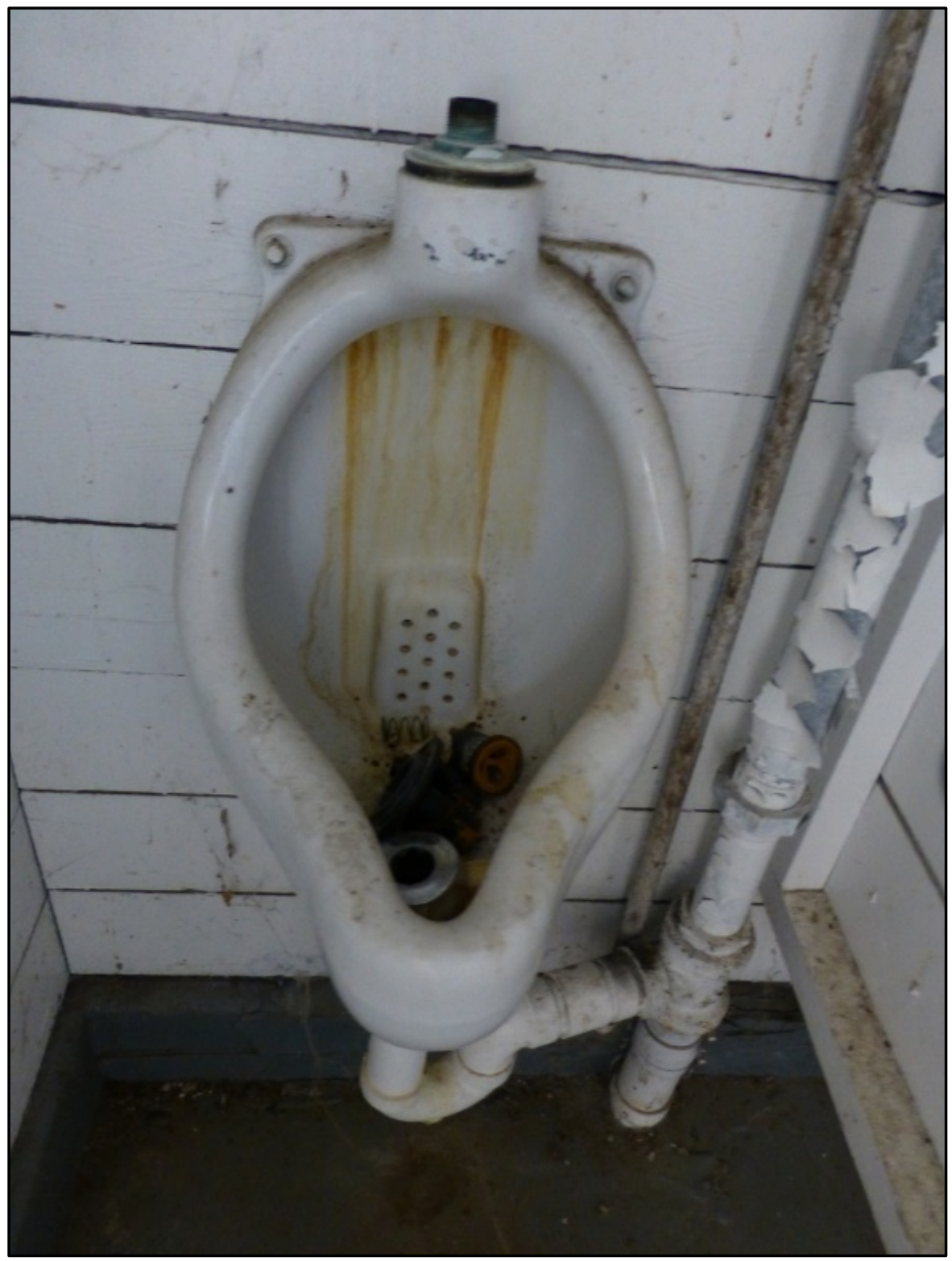

\subsection{Shower}

An original shower stall is intact in the toilet room in Bldg. 550 (see Figure 94). The condition appears to be POOR, as there is major water damage and possible mold. The blue-green portion of the shower may not be original. The wood-insert shower floor is also most likely not original; however, there may have been something similar to it used in the early days of Building 550. 
Figure 94. Looking at shower stall in Bldg. 550, 2018.

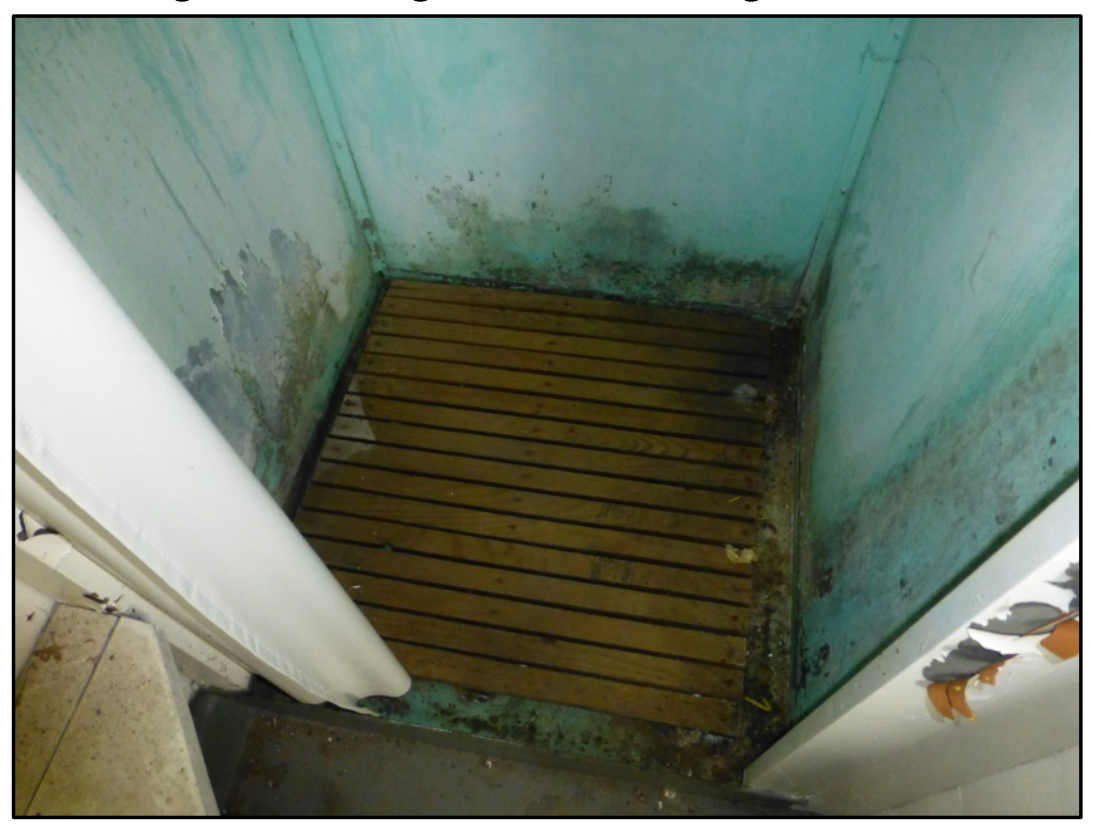

\subsection{Treatment measures}

It is rare to find a building constructed in a year as early as 1942 that contains original sinks and toilets. Due to this, there is minimal information regarding these fixtures. It is suggested that if the fixtures are repairable that they be repaired and maintained. The surfaces can be cleaned as normal.

There are no widely used treatment measures for porcelain, enamel, or zinc plumbing fixtures. The best manner to clean them and restore them to use is to use:

- Nonabrasive sponge

- Dishwashing soap

- Bar Keepers Friend (liquid)

- White vinegar

- Bleach (for white porcelain ONLY)

- Oxygen bleach or hydrogen peroxide

- Vinegar or lemon juice

- Naval jelly

- Paper towels

- Spray bottle

- Dish towel

- Muscles. 


\section{Stage III - Wood Floors}

Wood was the most common material used for floors in the construction of the WWII buildings at Camp McCoy in 1942. An original wood floor is a character-defining feature in an historic building. As the wood ages and is exposed to different temperatures or conditions, abnormal gaps can appear. The boards can buckle and cup if the wood expands in any way. Of course, depending on a buildings use, wear and tear, stains and blemishes are common.

\subsection{Wood strip flooring}

In Bldg. 550, original wood flooring is in the living area of the building, including the squad room, the NCO room, and the officers' room (see Figure 95). The condition of the flooring is GOOD. There is some visible gapping noticed, which might be caused by cupping, where the outer sides of the boards curl upward which results in space between the boards. These floors lack proper finishing. Looking at where the floor meets the wall, the floor appears to be raised/sunk in some areas. 
Figure 95. Looking at wood flooring in Bldg. 550, 2018.

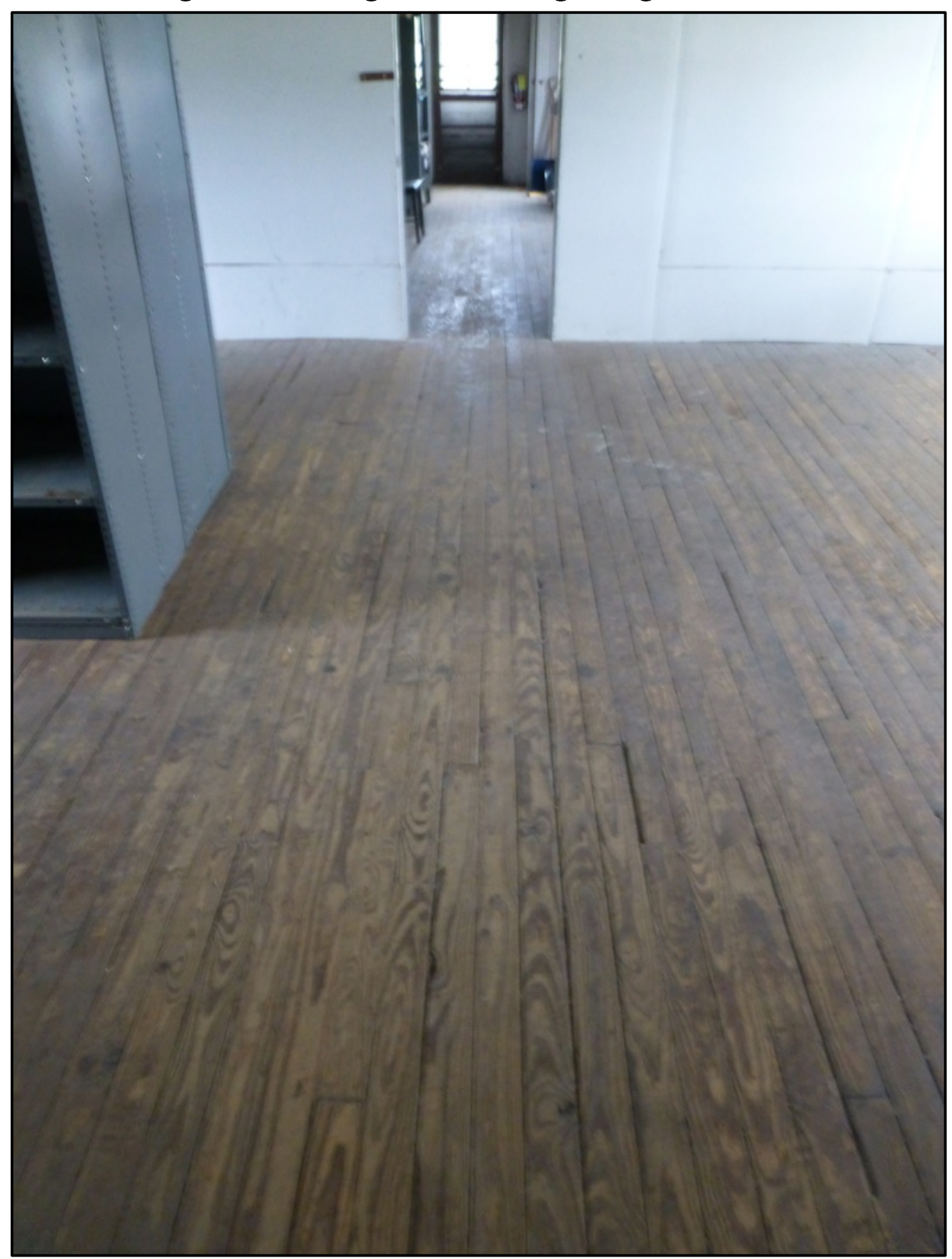

\subsection{Treatment measures}

Wood flooring is usually a key feature in an old building. In Bldg. 550, there is wood flooring, but it does not make up the entirety of the floor area throughout the building. Wood expands and shrinks over time as the temperature and air conditions fluctuate. Following are documents that include information on how to repair and protect aged wood floors. 
10.2.1 Stripping, staining, and polishing wood floors, ${ }^{30}$ GSA.gov

GSA U.S. General Services Administration

\title{
Stripping, Staining And Polishing Wood Floors
}

\author{
Procedure code: \\ 9550025 \\ Source: \\ National Capitol Region Specifications \\ Division: \\ Finishes \\ Section: \\ Wood Flooring \\ Last Modified: \\ 02/24/2012
}

PART 1---GENERAL

\subsection{SUMMARY}

A. This procedure includes guidance on stripping, staining and refinishing floors.

B. See 01100-07-S for general project guidelines to be reviewed along with this procedure. These guidelines cover the following sections:

1. Safety Precautions

2. Historic Structures Precautions

3. Submittals

4. Quality Assurance

5. Delivery, Storage and Handling

6. Project/Site Conditions

7. Sequencing and Scheduling

8. General Protection (Surface and Surrounding) These guidelines should be reviewed prior to performing this procedure and should be followed, when applicable, along with recommendations from the Regional Historic Preservation Officer (RHPO).

\subsection{PROJECT/SITE CONDITIONS}

A. Existing Conditions: Determine that surfaces to which finishes are to be applied are even, smooth, sound, clean, dry and free from defects affecting proper application. Correct or report defective surfaces to Contracting Officer.

30 Retrieved from: https://www.gsa.gov/real-estate/historic-preservation/historic-preservation-policytools/preservation-tools-resources/technical-documents. 


\section{PART 2---PRODUCTS}

\subsection{MANUFACTURERS}

A. Hillyard Chemical Company 800/365-1555

B. The Sherwin Williams Company 101 Prospect Ave. N.W. Cleveland, OH 44101 216/566-2000

\subsection{MATERIALS}

A. Sealer: Penetrating type, pliable, wood-hardening finish/sealer such as "Penetrating Seal \#21" (Hillyard Chemical Company), or approved equal.

B. Stain: Penetrating, permanent oil-based stain such as "Oil Stain" (Sherwin Williams), or approved equal, colored to match existing floor stain.

C. Clean, soft cloths

D. Fasteners: Nails and screws, select material, type, size and finish to match original installation.

E. Sandpaper: 3 grades, finest grade 00 .

\subsection{EQUIPMENT}

A. Hand-held Orbital Sanders (NO ROTARY OR DISK SANDERS)

B. Machine belt or orbital sander (NO ROTARY OR DISK SANDERS)

C. Stiff bristle brushes

\section{PART 3---EXECUTION}

\subsection{PREPARATION}

A. Surface Preparation:

1. Carefully remove any floor mounted elements and store in location selected by the Contracting Officer.

2. Protect floor borders from damage during sanding and other floor refinishing operations.

3. Machine sand the wood floor to remove stains, indentations and old finish. NOTE: BELT AND ORBITAL SANDERS SHOULD ONLY BE USED BY EXPERIENCED PERSONNEL. THEY WORK VERY QUICKLY AND IT IS EASY TO DAMAGE THE WOOD SUBSTRATE IF THEY ARE NOT USED CAREFULLY. For additional guidance on stripping wood floors and precautions to take when operating sanding equipment on wood, see 06300-02-R, 06400-10-R and 09910-01-S.

a. Sand floor in direction of wood grain. Remove only the amount of wood necessary to remove old finish but no more than $1 / 16$ " depth.

b. Use hand-held sanders at edges of floor.

c. Final sanding shall be with 00 grade sandpaper.

d. Vacuum clean, remove sanding dusted with tack cloth, and immediately apply stain finish.

e. Do not allow traffic on floor until final finishing is complete.

\subsection{ERECTION, INSTALLATION, APPLICATION}

A. Apply stain to wood floor to obtain a finish to match the original.

B. Apply floor sealer (two coats) in accordance with manufacturer's printed instructions, including machine buffing with steel wool as recommended by the manufacturer.

C. Reinstall floor mounted accessories in original locations. Protect wood flooring during reinstallation.

D. For guidance on cleaning and maintaining woodwork and wood floors, see 06400-02-P, 06400-01-R, and 0640001-P. 


\subsubsection{Spot cleaning stains on wood floors, ${ }^{31} 2016$ (GSA.gov).}

\section{Spot Cleaning Stains on Wood Floors}

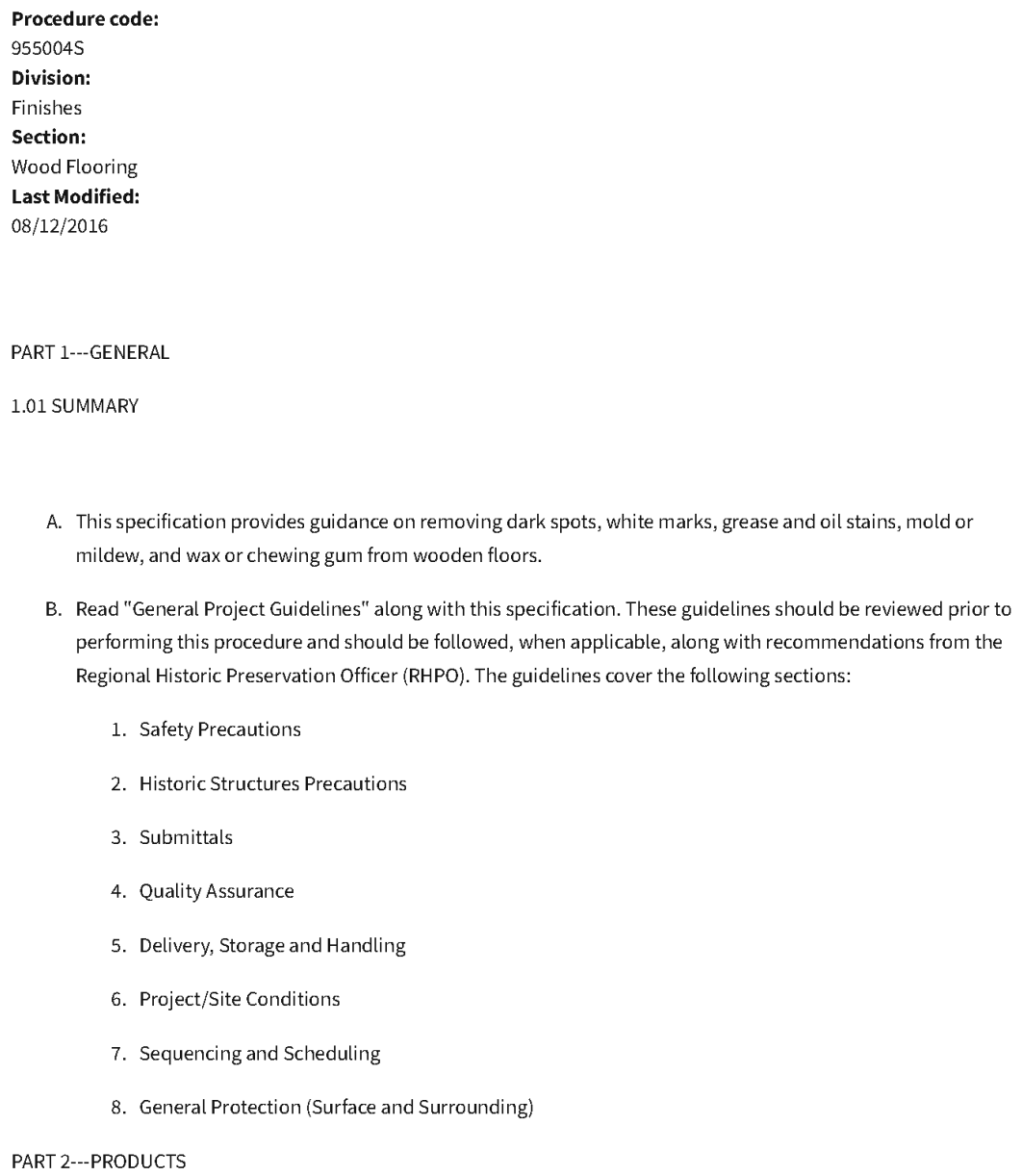

31 Retrieved from: https://www.gsa.gov/real-estate/historic-preservation/historic-preservation-policytools/preservation-tools-resources/technical-documents. 


\subsection{MATERIALS}

NOTE: Chemical products are sometimes sold under a common name. This usually means that the substance is not as pure as the same chemical sold under its chemical name. The grade of purity of common name substances, however, is usually adequate for stain removal work, and these products should be purchased when available, as they tend to be less expensive. Common names are indicated below by an asterisk ( $\left.{ }^{*}\right)$.

A. Steel wool pads (Fine Grade 000).

B. Mineral Spirits:

1. A petroleum distillate that is used especially as a paint or varnish thinner.

2. Other chemical or common names include Benzine* (not Benzene); Naphtha*; Petroleum spirits*; Solvent naphtha*.

3. Potential Hazards: TOXIC AND FLAMMABLE.

4. Safety Precautions:

a. AVOID REPEATED OR PROLONGED SKIN CONTACT.

b. ALWAYS wear rubber gloves when handling mineral spirits.

c. If any chemical is splashed onto the skin, wash immediately with soap and water.

5. Available from construction specialties distributor, hardware store, paint store, or printer's supply distributor.

C. Sealer.

D. Solvent-based floor wax.

E. Household Vinegar:

1. Potential Hazards: CORROSIVE TO CONCRETE, STEEL, WOOD AND GLASS.

2. Available from grocery store or supermarket.

3. Vinegar contains about $4 \%$ acetic acid and may be suitable for some purposes requiring acetic acid.

F. Household bleach:

1. Other chemical or common names include bleaching solution ${ }^{*}$, laundry bleach ${ }^{*}$, sodium hypochlorite $(\mathrm{NaOCl})$ and solution of chlorinated soda*.

2. Potential Hazards: CAUSTIC TO FLESH.

3. Available from chemical supplier, grocery store or supermarket, hardware store or janitorial supply distributor.

G. Oxalic acid, aka $(\mathrm{COOH}) 2$ or $\mathrm{C} 2 \mathrm{H} 2 \mathrm{O} 4$ : 
1. A poisonous strong acid that occurs in various plants as oxalates and is used especially as a bleaching or cleaning agent and in making dyes.

2. Other chemical or common names include dibasic acid, ethanedioic acid, acid of sugar*.

3. Potential Hazards: TOXIC; CORROSIVE TO CONCRETE, STEEL, WOOD AND GLASS.

4. Available from chemical supplier, dry cleaning supply distributor, drugstore or pharmaceutical supply distributor, hardware store, or photographic supply distributor (not camera shop). (Often sold under a manufacturer's brand name; the chemical name may appear on the label.)

H. Paper towels and clean, soft cotton cloths.

I. Plastic bags.

J. Ice cubes.

K. Clean, potable water.

2.02 EQUIPMENT

A. Industrial-sized floor buffer.

B. Plastic spatula.

PART 3---EXECUTION

3.01 PREPARATION

A. Protection:

1. Carefully read manufacturer s instructions for any chemical to be used for cleaning. Follow recommendations for safety and handling as well as application.

2. Be sure to provide adequate ventilation when using solvents.

B. Surface Preparation: ALWAYS test selected method of cleaning on a small area before beginning the actual job.

3.02 ERECTION, INSTALLATION, APPLICATION

NOTE: USE CAUTION WHEN USING SCOURING POWDER AS IT CAN PERMANENTLY SCRATCH WOOD FLOORS. DO NOT USE WATER-BASED CLEANING SOLUTIONS OR CLEANERS THAT REQUIRE RINSING WITH WATER. USE A SOLVENT OR SOLVENT-BASED CLEANING WAX.

A. For Dark Spots:

1. Rub area with steel wool and mineral spirits.

2. Then, wet a rag with household vinegar and apply to the stain. Allow to sit on dark spot for a few minutes.

3. Repeat this process if it seems to lighten the stain. 
4. If the spot does not lighten, mix a 50/50 solution of household bleach and water and apply to the stain.

5. If the spot still does not lighten, then apply oxalic acid to the center of the spot. Avoid getting bleach on the surrounding wood. Several applications may be necessary.

6. When the spot has disappeared, neutralize the bleach with vinegar and allow to dry. Sand, stain and refinish to match the original color.

B. For White Water Marks:

1. White marks are usually a stain in the finish, not in the wood

2. Rub the mark with very fine steel wool and apply paste wax.

C. For Grease and Oil Stains:

1. Blot stain with clean towels.

2. Saturate stain with dry cleaning fluid. Check appearance every few minutes.

D. For Mold or Mildew:

1. Wipe area with rag soaked in household bleach.

2. Provide adequate ventilation in future to eliminate damp, stagnant air conditions that can result in the growth of mold and mildew.

E. For Wax or Chewing Gum:

1. Put ice cubes in a plastic bag and place on top of the wax or chewing gum. Allow it to sit for a few minutes.

2. When the material has hardened, scrape it away with a plastic spatula and recoat the floor with floor polish.

3.03 ADJUSTING/CLEANING

Upon completion of stain removal, rinse the area thoroughly, and allow to dry. Repair any areas where finish has been removed. 


\title{
10.2.3 Repairing cupped floorboards, ${ }^{32} 2016$ (GSA.gov)
}

\section{Repairing Cupped Floorboards}

\author{
Procedure code: \\ 9560055 \\ Source: \\ National Oak Flooring Manufacturer's Association \\ Division: \\ Finishes \\ Section: \\ Wood Strip Flooring \\ Last Modified: \\ 08/04/2016
}

PART 1---GENERAL

1.01 SUMMARY

A. This procedure includes guidance on repairing cupped floorboards.

B. Cupping of hardwood floors is caused by one side of the board gaining or losing moisture faster than the other side. Some cupping, especially in wide plank floors, is considered normal. It is usually barely noticeable. More severe cupping is of two types:

C. Convex cupping ("crowning"): When the center of

1. the board is higher than the edges of the board.

2. Concave cupping: When the edges of the board are higher than the center of the board.

3. Cupping may result from the inability of moisture to evaporate through the boards. In the case of concave cupping, the presence of an impervious surface finish on the floor may prohibit the transmission of moisture, causing it to build-up on the back side of the board.

4. See 01100-07-S for general project guidelines to be

5. reviewed along with this procedure. These guidelines cover the following sections:

a. Safety Precautions

b. Historic Structures Precautions

c. Submittals

d. Quality Assurance

e. Delivery, Storage and Handling

f. Project/Site Conditions

g. Sequencing and Scheduling

h. General Protection (Surface and Surrounding)

These guidelines should be reviewed prior to performing this procedure and should be followed, when applicable,along with recommendations from the Regional Historic Preservation Officer (RHPO).

1.02 REFERENCES

32 Retrieved from: https://www.gsa.gov/real-estate/historic-preservation/historic-preservation-policytools/preservation-tools-resources/technical-documents. 
A. National Wood Flooring Association Chesterfield, MO

PART 2---PRODUCTS

2.01 MANUFACTURERS

A. Moisture Meters: Moisture Register Products Div. Aqua Measure Instrument $\mathrm{Co}$.

B. Delmhorst Instrument Company

E, Towaco, NJ

C. Lignomat USA, Ltd.

Portland, OR

D. Wagner Electronic Inc.

Jeffersontown, $\mathrm{KY}$

E. Hygrometers and Sling Psychometers:Fisher Scientific

Pittsburgh, PA

F. Forestry Suppliers, Inc. Jackson, MS

\subsection{MATERIALS}

A. Wood screws

B. Soap or paraffin to lubricate screw

C. Commercial wood putty

D. Headless steel pins

E. Wood floor refinishing materials (see 06400-10-R)

F. Clean, white towels

G. Clean, potable water

\subsection{EQUIPMENT}

A. Moisture meter or sling psychrometer

B. Dehumidifier

C. Screwdriver

D. Hammer

E. Floor refinishing equipment (see 06400-10-R)

PART 3---EXECUTION

\subsection{ERECTION, INSTALLATION, APPLICATION}

A. Correct moisture levels by removing the source of excess moisture.

B. Use a dehumidifier or check outside drainage to see that water is draining properly away from the building.

C. Regrade if necessary.

D. Allow the boards to dry out (this may take several weeks or months).

E. Take moisture readings bi-weekly or bi-monthly to accurately determine humidity levels. Use a sling psychrometer or digital thermometer hygrometer.

NOTE: Do not proceed with any repairs until the moisture readings are balanced between the face and back of the board for at least 30 days.

NOTE: Floors with a surface finish will dry out much slower as they tend to respond much slower to moisture changes in the environment.

F. If the cupped boards dry out, they should return to their normal position.

$G$. If the boards flatten when fully dried, resecure loose boards to the subfloor.

H. For floors installed using nails:

a. Check for loose nails. These may be quickly located by identifying squeaks in the floor or loose boards.

b. Face-nail loose boards or refasten with wood screws. See also 09560-04-R for guidance on silencing squeaking wood floors.

I. For Floors Installed Using Mastic: 
1. Check adhesion of floorboards to subfloor. Walk across the floor and listen to the sounds made. If a popping sound is heard, or if the floor sounds hollow when tapped, adhesion is probably lost.

2. Remove affected boards. Some adhesives such as asphalt cut-back mastic can be reactivated by lightly spraying it with kerosene. Consult manufacturer for recommendations.

-OR-

Drive headless steel pins through the affected boards and into a wood or concrete subfloor.

J. Fill cracks with commercial wood putty. See 09560-02-R for guidance on repairing small holes and cracks in wood floors.

A. Reapply surface finish.

-OR-

Buff with 00 steel wool, clean and rewax.

NOTE: IF A POLYURETHANE FINISH WAS USED, RECOAT ONLY IF THE FLOOR HAS NOT BEEN WAXED.

$K$. If the floor remains cupped after drying, it must be completely resanded and refinished (see 06400-10-R for guidance).

NOTE: Be sure to fill cracks, check fasteners and make any repairs before sanding.

L. A severely warped or buckled floorboard which cannot be worked back into place, will have to be replaced, see 09560-01-R "Replacing Damaged Floorboards" for guidance. 


\title{
10.2.4 Replacing damaged floorboards, ${ }^{33} 2017$ (GSA.gov).
}

\section{Replacing Damaged Floorboards}

\author{
Procedure code: \\ 9560015 \\ Source: \\ NPS Southeast Regional Office \\ Division: \\ Finishes \\ Section: \\ Wood Strip Flooring \\ Last Modified: \\ $12 / 26 / 2017$
}

\section{PART 1---GENERAL}

\subsection{SUMMARY}

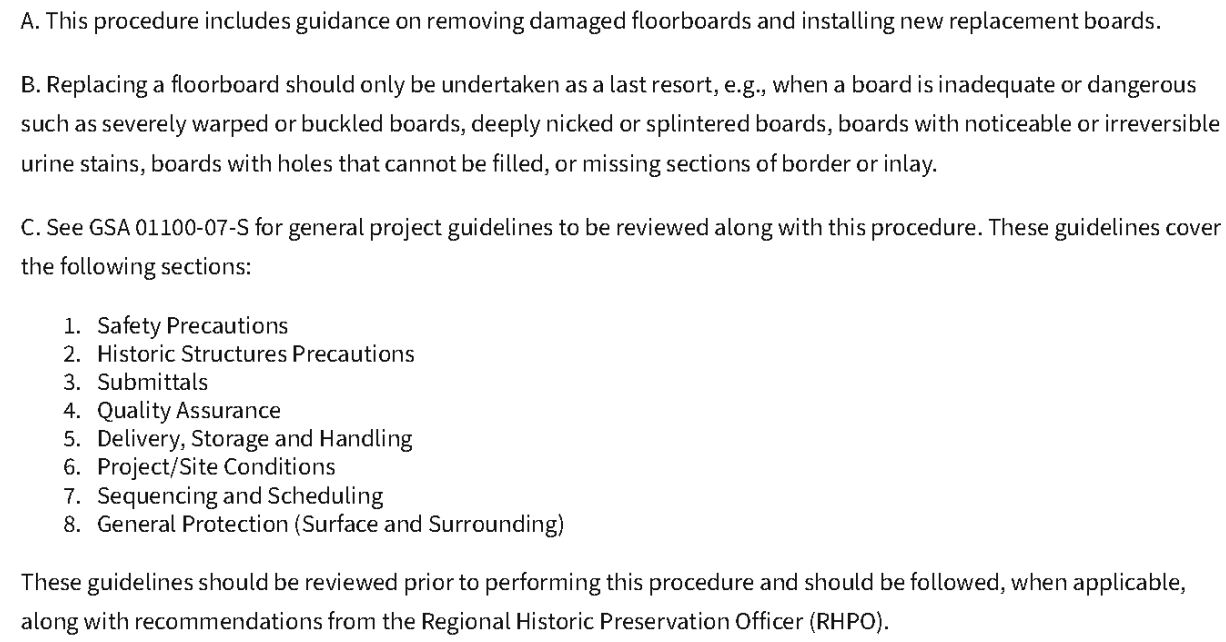

33 Retrieved from: https://www.gsa.gov/real-estate/historic-preservation/historic-preservation-policytools/preservation-tools-resources/technical-documents. 


\subsection{SYSTEM DESCRIPTION}

A. A wood floor surface can be either a series of connected planks or parquet (small wood pieces arranged in decorative patterns). The wood used is either plain sawn or quarter sawn. Plank flooring, the more common type, is assembled by joining: butt joint, tongue and groove, shiplap, doweled or spline. Wood floors are usually secured to the under structure by countersinking nails, blind-nailing, or screwing and plugging.

B. A wood floor surface in proper condition does not sag, is not inadvertently stained, is free from protruding nails, and is not warped.

\subsection{DELIVERY, STORAGE AND HANDLING}

A. Storage and Protection:

1. Every effort must be made to use and reuse materials that are original to the structure. When removed from their rightful place, these materials must be stored under cover inside the building where they cannot be damaged.

2. If many pieces are to be removed, they must be marked inconspicuously in a consistent manner as to their location originally.

3. If salvage material is to be used, treat it as the original material with regards to its storage.

4. If new material must be used, keep it dry during delivery, storage and handling.

5. Do not allow materials to be stored in contact with damp surfaces.

\section{PART 2---PRODUCTS}

\subsection{MANUFACTURERS (The following, or approved equal)}

A. Craftsman Lumber Co.

2.02 MATERIALS

A. Replacement board (to match existing wood type, grain, etc.) from a salvage yard, new lumber yard, or inconspicuous place in building.

NOTE: In buildings where tenant areas were originally finished with wood plank or parquet, the wood is often retained as a sub-floor for carpet. Such concealed areas may be a source of replacement flooring for areas of exposed wood.

B. Wood for shims (no shingles)

C. Wood putty to fill holes

D. Colors-in-oils or residue from stain container to stain putty to match

E. Flooring nails

\subsection{EQUIPMENT}

A. Keyhole saw, circular saw, or mallet and chisel to remove damaged board

B. Drill to make a pilot hole for the keyhole saw

C. Nailset 
D. Tools for accurate measurement

E. Carpet scrap or newspaper and pounding block for knocking new piece into place

\section{PART 3---EXECUTION}

\subsection{EXAMINATION}

A. Inspect for wear in the surface such as chips or gouges. If the wear is minimal, holes can be filled and the surface restored.

B. Inspect for the signs of insect infestation such as mold, fungus, bore holes, and sawdust piles. Probe the wood with an ice pick or thin knife blade to determine the existence of rot.

\subsection{ERECTION, INSTALLATION, APPLICATION}

A. Removing a damaged board:

NOTE: THE DAMAGED BOARD WILL HAVE TO BE DESTROYED TO REMOVE IT.

1. If the gap between boards is wide enough, remove the tongue of the damaged board with a chisel and lift board out. -OR-

2. Remove board with a saw:

a. Drill a hole, next to the joist edge, large enough for a keyhole saw to be inserted. DO NOT DRILL THROUGH THE SUBFLOOR. $b$. With the saw, make a cut along the width of the board and along the length of the section to be removed. c. Carefully pry the board out, protecting adjacent wood. d. If a circular saw is used, set the blade depth to the thickness of the finished floor. Use a carbide flooring blade that will also cut through nails. CAUTION: Do not cut all the way across to the edge of adjacent floor boards. A loss of control can do irreparable damage to adjacent boards.

b. Use a chisel to finish the cut.

$-\mathrm{OR}-$

3. Remove board with a mallet and chisel:

a. Cut along the width of the board to the nearest joist on either side of damage with the beveled edge of the chisel facing the damage. Make sure that the joints in floorboards remain staggered.

b. To free the board from nails, channel cut a wedge from each end holding the chisel at a 30 degree angle with the bevel side down, or drive nails through board with nailset

c. Remove the center section down the face of the board. The other pieces should then come out easily.

B. Installing a new board:

1. Square up the edges of the hole before inserting replacement board.

2. Measure the new board to fit exactly. If it is tongue and groove, remove the bottom shoulder of the groove. Shim if necessary.

3. If no subfloor exists, add blocking to joist below to support new floorboard.

4. Knock the new board into place protecting the surface with a carpet scrap or newspaper and a pounding block.

5. Face nail the board to the subfloor or nail board ends into joists or attached nailing blocks.

6. Fill the nail holes with wood filler stained to match floor.

a. Add filler in layers and allow to dry between each layer.

b. To stain wood filler use either colors-in-oils or the settled pigment from the bottom of a stain container. When staining to match, go darker than the original color rather than lighter. 


\section{Stage III - Brick and Mortar}

The majority of the WWII buildings had a brick chimney stack projecting above the roofline or a detached brick chimney stack adjacent to the building.

There is minimal masonry in Bldg. 550. The original chimney is located toward the center of the building right off the stairs into the apparatus room. The chimney is accessible from the heater room.

\subsection{Chimney}

This base of the chimney in Figure 96 shows the FAIR condition of the brick. There is discoloration in some areas, and some spacing in the mortar. Brick of this age is prone to problems with the pointing.

Figure 96. Looking at base of the brick chimney located on the east side of the apparatus room, 2018.

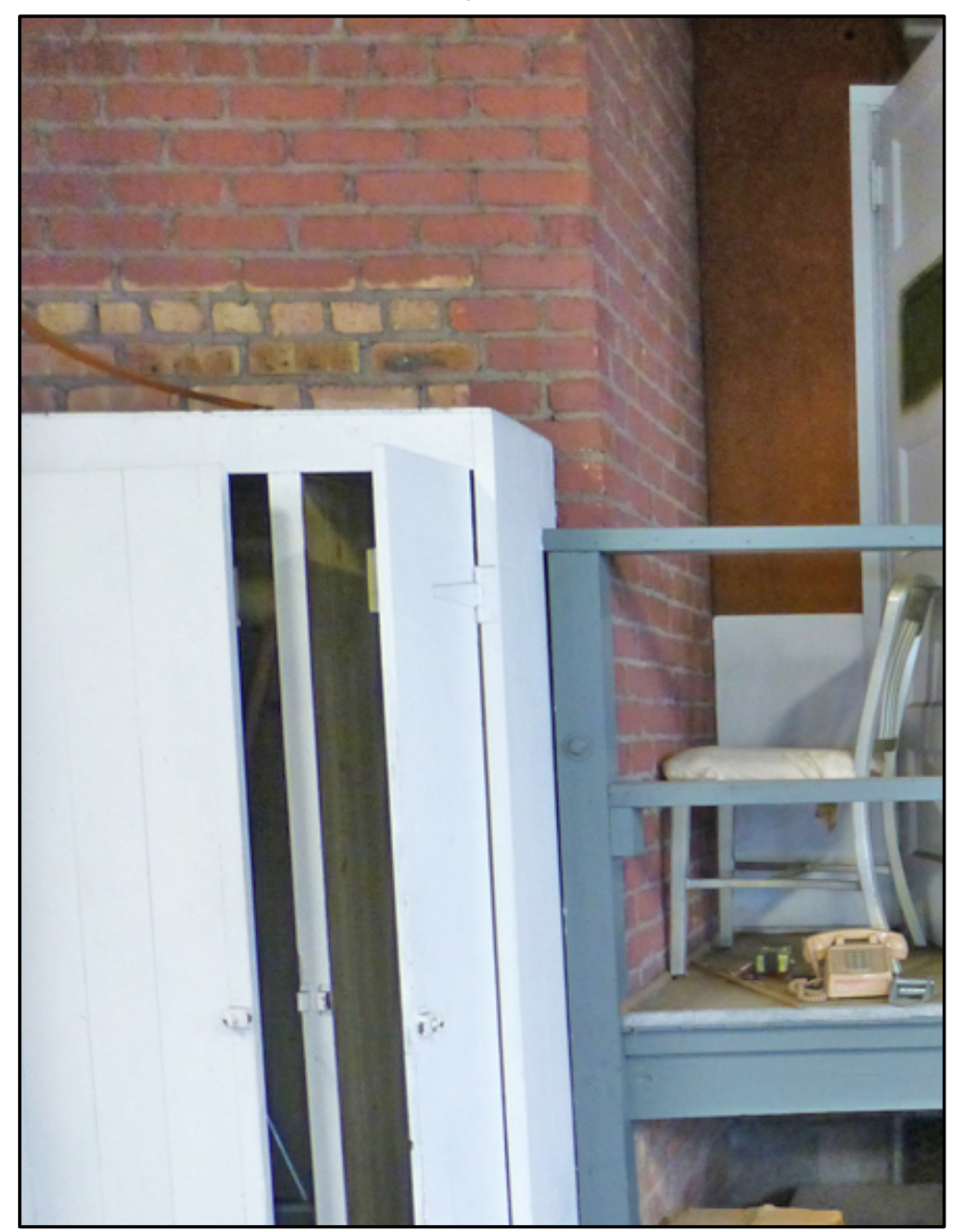


Bldg. 550 used to have a full brick chimney extending out of the roof, as seen in Figure 97. In later years, the exterior portion of the chimney was removed and replaced with a metal attachment, seen in Figure 98. The original brick still extends from the floor to the roof. Even though this brick feature is character defining, there is no need to re-construct the chimney to match the original plans.

Figure 97. Plan Number $800-800$ detail of original brick chimney extending out of the roof, 1942.

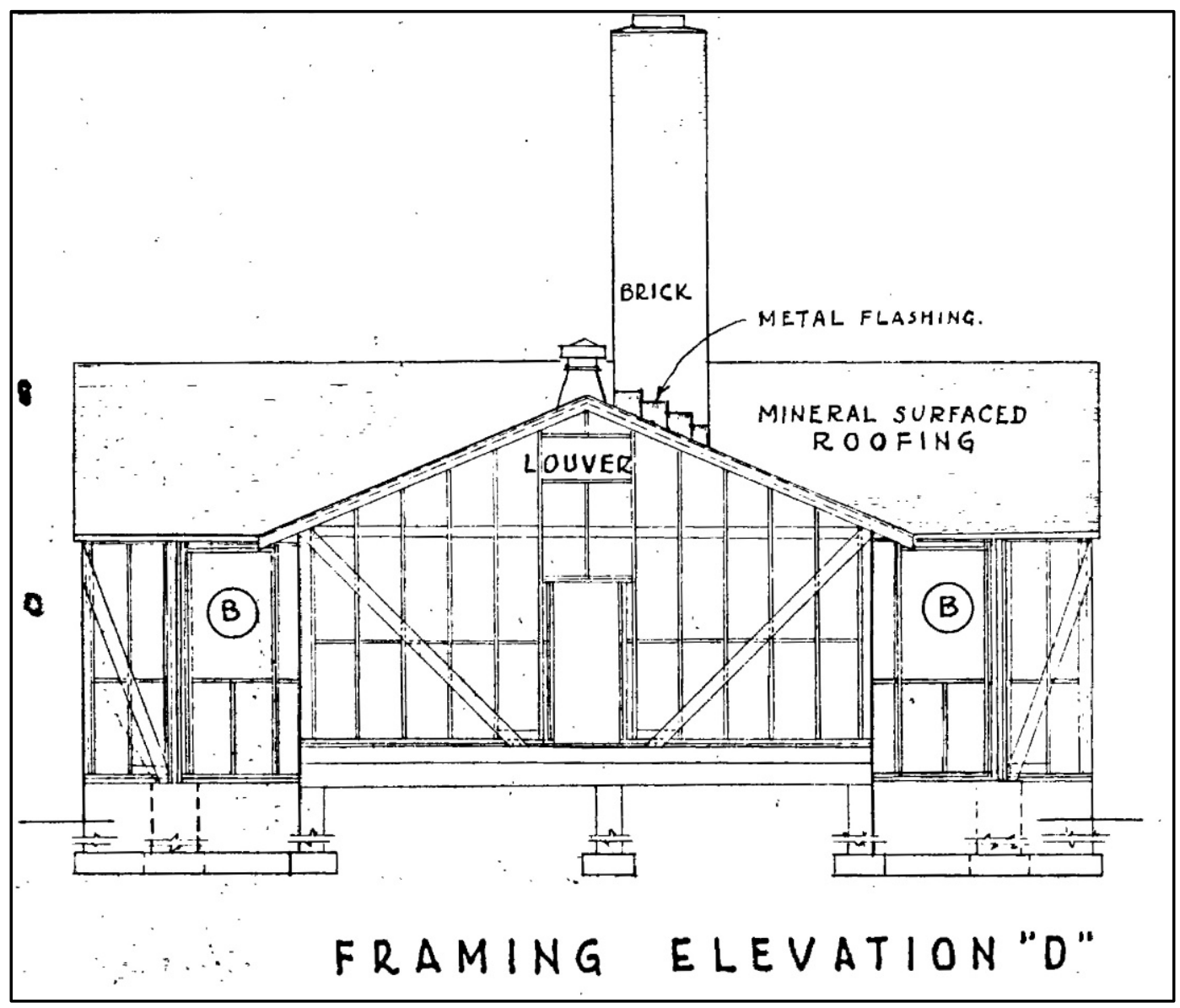

Source: Fort McCoy DPW. 


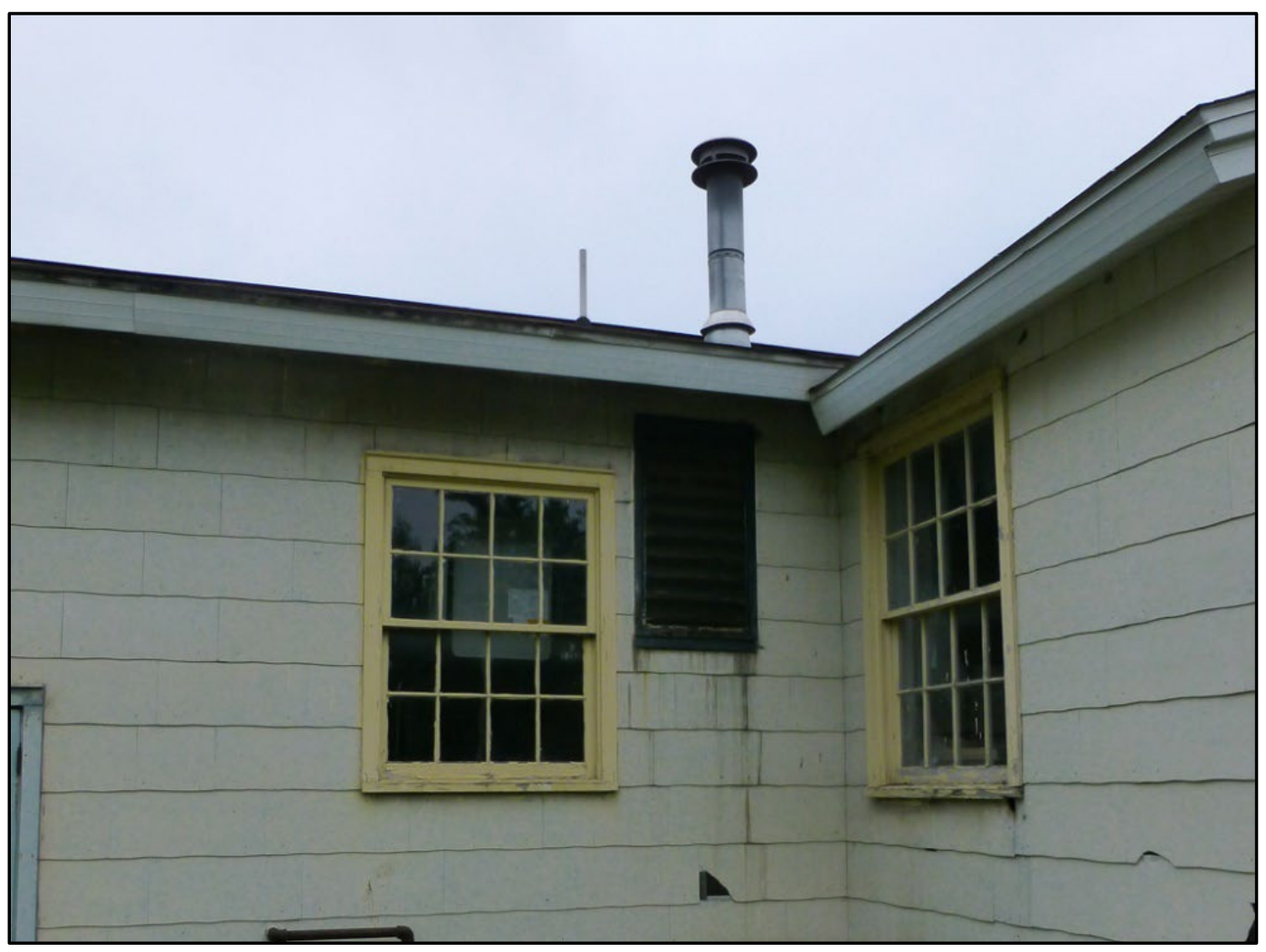

\subsection{Treatment measures}

There is minimal usage of brick masonry in Bldg. 550. The chimney consists of brick that was installed in 1942; however, the chimney was modified at a later date. The brick that is still present can be repaired and/or maintained based on the guidance in the following documents. 


\subsubsection{Preservation Brief $\mathbf{2}^{34}$ - Repointing joints in historic masonry buildings, 1998 (NPS.gov).}

Technical Preservation Services

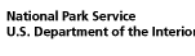

PRESERVATION BRIEFS

2

\section{Repointing Mortar Joints in Historic Masonry Buildings}

Robert C. Mack, FAIA, and John P. Speweik

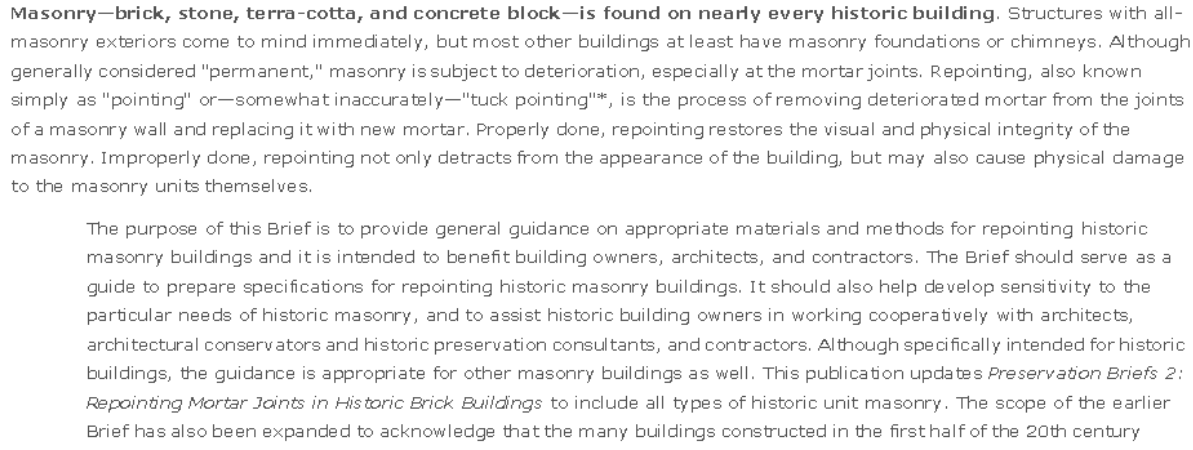

34 Retrieved from: https://www.nps.gov/tps/how-to-preserve/briefs.htm. 
iw historic and eligible for listing in the National Register of Historic Places, and that they may have been originally constructed th portland cement mortar.

* Tuckpainting technically describes a primanily decorative application of a raised mortar jaint or lime putty jaint on top of flush mortar joints.

\section{Historical Background}

Mortar consisting primarily of lime and sand has been used as an integral part of masonry structures for thousands of years. Up until about the mid-19th century, lime or quicklime (sometimes called lump lime) was delivered to construction sites, where it had to be slaked, or combined with water. Mixing with water caused it to boil and resulted in a wet lime putty that was left to mature in a pit or wooden box for several weeks, up to a year. Traditional mortar was made from lime putty, or slaked lime, combined with local sand, generally in a ratio of 1 part lime putty to 3 parts sand by volume. often other ingredients, such as crushed marine shells (another source of lime), brick dust, clay, natural cements, pigments, and even animal hair were also added to mortar, but the basic formulation for lime putty and sand mortar remained unchanged for centuries until the advent of portland cement or its forerunner, Roman cement, a natural, hydraulic cement.

Portland cement was patented in Great Britain in 1824. It was named after the stone from Portland in Dorset which it resembled when hard. This is a fast-curing, hydraulic cement which hardens under water. Portland cement was first manufactured in the United States in 1872, although it was imported before this date. But it was not in common use throughout the country until the early 20th century. Up until the turn of the century portland cement was considered primarily an additive, or "minor ingredient" to help accelerate mortar set time. By the 1930s, however, most masons used a mix of equal parts portland cement and lime putty. Thus, the mortar found in masonry structures built between 1873 and 1930 can range from pure lime and sand mixes to a wide variety of lime, portland cement, and sand combinations.

In the 1930 s more new mortar products intended to hasten and simplify masons' work were introduced in the U.S. These included masonry cement, a premixed, bagged mortar which is a combination of portland cement and ground limestone, and hydrated lime, machine-slaked lime that eliminated the necessity of slaking quicklime into putty at the site.

\section{Identifying the Problem Before Repointing}

The decision to repoint is most often related to some obvious sign of deterioration, such as disintegrating mortar, cracks in mortar joints, loose bricks or stones, damp walls, or damaged plasterwork. It is, however, erroneous to assume that repointing alone will solve deficiencies that result from other problems. The root cause of the deterioration-leaking roofs or gutters, differential settlement of the building, capillary action causing rising damp, or extreme weather exposure-should always be dealt with prior to beginning work.

Without appropriate repairs to eliminate the source of the problem, mortar deterioration will continue and any repointing will have been a waste of time and money.

\section{Use of Consultants}

Because there are so many possible causes for deterioration in historic buildings, it may be desirable to retain a consultant, such as a historic architect or architectural conservator, to analyze the building. In addition to determining the most appropriate solutions to the problems, a consultant can prepare specifications which reflect the particular requirements of each job and can provide oversight of the work in progress. Referrals to preservation consultants frequenty can be obtained from State Historic Preservation offices, the American

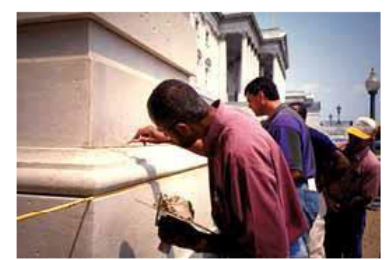

Masons practioe using lime putty mortar to repair historic marble. Photo: NPS files. Institute for Conservation of Historic and Artistic Works (AIC), the Association for Preservation Technology (APT), and local chapters of the American Institute of Architects (AIA)

\section{Finding an Appropriate Mortar Match}

Preliminary research is necessary to ensure that the proposed repointing work is both physically and visually appropriate to the building. Analysis of unweathered portions of the historic mortar to which the new mortar will be matched can suggest appropriate mixes for the repointing mortar so that it will not damage the building because it is excessively strong or vapor impermeable.

Examination and analysis of the masonry units-brick, stone or terra cotta-and the techniques used in the original construction will assist in maintaining the building's historic appearance. A simple, non- technical, evaluation of the masonry units and 
mortar can provide information concerning the relative strength and permeability of each-critical factors in selecting the repointing mortar-while a visual analysis of the historic mortar can provide the information necessary for developing the new mortar mix and application techniques.

Athough not crucial to a successful repointing project, for projects involving properties of special historic significance, a mortar analysis by a qualified laboratory can be useful by providing information on the original ingredients. However, there are limitations with such an analysis, and replacement mortar specifications should not be based solely on laboratory analysis. Analysis requires interpretation, and there are important factors which affect the condition and performance of the mortar that cannot be established through laboratory analysis. These may include: the original water content, rate of

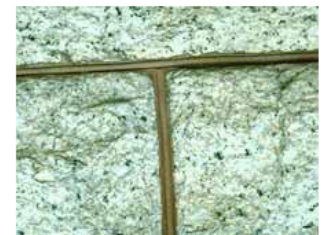

This late 19th century granite has recently been repointed with the joint matched to the original. Photo: NPS files. curing, weather conditions during original construction, the method of mixing and placing the mortar, and the cleanliness and condition of the sand. The most useful information that can corre out of laboratory analysis is the identification of sand by gradation and color. This allows the color and the texture of the mortar to be matched with some accuracy because sand is the largest ingredient by volume.

In creating a repointing mortar that is compatible with the masonry units, the objective is to achieve one that matches the historic mortar as closely as possible, so that the new material can coexist with the old in a sympathetic, supportive and, if necessary, sacrificial capacity. The exact physical and chemical properties of the historic mortar are not of major significance as long as the new mortar conforms to the following criteria:

- The new mortar must match the historic mortar in color, texture and tooling. (If a laboratory analysis is undertaken, it may be possible to match the binder components and their proportions with the historic mortar, if those materials are available.)

- The sand must match the sand in the historic mortar. (The color and texture of the new mortar will usually fall into place if the sand is matched successfully.)

- The new mortar must have greater vapor permeability and be softer (measured in compressive strength) than the masonry units.

- The new mortar must be as vapor permeable and as soft or softer (measured in compressive strength) than the historic mortar. (Softness or hardness is not necessarily an indication of permeability; old, hard lime mortars can still retain high permeability.)

\section{Mortar Analysis}

Methods for analyzing mortars can be divided into two broad categories: wet chemical and instrumental. Many laboratories that analyze historic mortars use a simple wet-chemical method called acid digestion, whereby a sample of the mortar is crushed and then mixed with a dilute acid. The acid dissolves all the carbonate-containing minerals not only in the binder, but also in the aggregate (such as oyster shells, coral sands, or other carbonate-based materials), as well as any other acid-soluble materials. The sand and fine-grained acid-insoluble material is left behind. There are several variations on the simple acid digestion test. One involves collecting the carbon dioxide gas given off as the carbonate is digested by the acid; based on the gas volume the carbnate content of the mortar can be accurately determined (Jedrze jewska, 1960). Simple acid digestion methods are rapid, inexpensive, and easy to perform, but the information they provide about the original composition of a mortar is limited to the color and texture of the sand. The gas collection method provides more information about the binder than a simple acid digestion test.

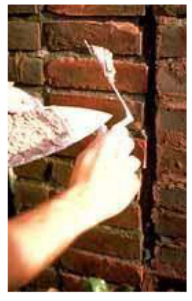
This mortar is the proper consistency for repointing historic brick.
Photo: John P. Speweik.

Instrumental analysis methods that have been used to evaluate mortars include polarized light or thin-section microscopy, scanning electron microscopy, atomic absorption spectroscopy, X-ray diffraction, and differential thermal analysis. Al instrumental methods require not only expensive, specialized equipment, but also highly-trained experienced analysts. However, instrumental methods can provide much more information about a mortar. Thin-section microscopy is probably the most commonly used instrumental method. Examination of thin slices of a mortar in transmitted light is often used to supplement acid digestion methods, particularly to look for carbonate-based aggregate. For example, the new ASTM test method, ASTM C 1324-96 "Test Method for Examination and Analy sis of Hardened Mortars" which was designed specifically for the analysis of modern lime-cement and masonry cement mortars, combines a complex series of wet chemical analyses with thin-section microscopy.

The drawback of most mortar analysis methods is that mortar samples of known composition have not been analyzed in order to evaluate the method. Historic mortars were not prepared to narrowly defined specifications from materials of 
uniform quality; they contain a wide array of locally derived materials combined at the discretion of the mason. While a particular method might be able to accurately determine the original proportions of a lime-cement-sand mortar prepared from modern materials, the usefulness of that method for evaluating historic mortars is questionable unless it has been tested against mortars prepared from materials more commonly used in the past.

\section{Properties of Mortar}

Mortars for repointing should be softer or more permeable than the masonry units and no harder or more impermeable than the historic mortar to prevent damage to the masonry units. It is a common error to assume that hardness or high strength is a measure of appropriateness, particularly for lime-based historic mortars. Stresses within a wall caused by expansion, contraction, moisture migration, or settlement must be accommodated in some manner; in a masonry wall, these stresses should be relieved by the mortar rather than by the masonry units. A mortar that is stronger in compressive strength than the masonry units will not "give," thus causing stresses to be relieved through the masonry units-resulting in permanent damage to the masonry, such as cracking and spalling, that cannot be repaired easily.

While stresses can also break the bond between the mortar and the masonry units, permitting water to penetrate the resulting hairline cracks, this is easier to correct in the joint through repointing than if the break occurs in the masonry units.

Permeability, or rate of vapor transmission, is also critical. High lime mortars are more permeable than denser cement mortars. Historically, mortar acted as a bedding material-not unlike an expansion joint-rather than a "glue" for the masonry units, and moisture was able to migrate through the mortar joints rather than the masonry units. When moisture evaporates from the masonry it deposits any soluble salts either on the surface as efflorescence or below the surface as sub forescence. While salts deposited on the surface of masonry units are usually relatively harmless, salt crystallization within a masonry unit creates pressure that can cause parts of the outer surface to spall off or delaminate. If the mortar does not permit moisture or moisture vapor to migrate out of the wall and evaporate, the result will be damage to the

masonry units.

\section{Components of Mortar}

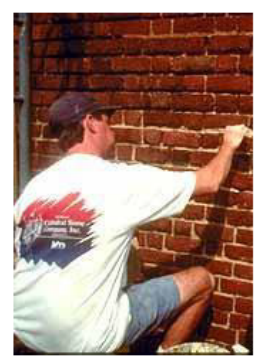

\section{Sand}

Sand is the largest component of mortar and the material that gives mortar its distinctive color, texture and cohesiveness Sand must be free of impurities, such as salts or clay. The three key characteristics of sand are: particle shape, gradation and void ratios.

When viewed under a magnifying glass or low-power microscope, particles of sand generally have either rounded edges, such as found in beach and river sand, or sharp, angular edges, found in crushed or manufactured sand. For repointing mortar, rounded or natural sand is preferred for two reasons. It is usually similar to the sand in the historic mortar and provides a better visual match. It also has better working qualities or plasticity and can thus be forced into the joint more easily, forming a good contact with the remaining historic mortar and the surface of the adjacent masonry units. Al though manufactured sand is frequenty more readily available, it is usually possible to locate a supply of rounded sand.

The gradation of the sand (particle size distribution) plays a very important role in the durability and cohesive properties of a mortar. Mortar must have a certain percentage of large to small particle sizes in order to deliver the optimum performance. Acceptable guidelines on particle size distribution may be found in ASTM C 144 (American Society for Testing and Materials). However, in actuality, since neither historic nor modern sands are always in compliance with ASTM C 144, matching the same particle appearance and gradation usually requires sieving the sand.

A scoop of sand contains many small voids between the individual grains. A mortar that performs well fills all these small voids with binder (cement/lime combination or mix) in a balanced manner. Well-graded sand generally has a 30 per cent void ratio by volume. Thus, 30 per cent binder by volume generally should be used, unless the historic mortar had a different binder: aggregate ratio. This represents the $1: 3$ binder to sand ratios often seen in mortar specifications.

For repointing, sand generally should conform to ASTM C 144 to assure proper gradation and freedom from impurities; some variation may be necessary to match the original size and gradation. Sand color and texture also should match the original as closely as possible to provide the proper color match without other additives.

\section{Lime}

Mortar formulations prior to the late-19th century used lime as the primary binding material. Lime is derived from heating limestone at high temperatures which burns off the carbon dioxide, and turns the limestone into quicklime. There are three 
types of limestone-calcium, magnesium, and dolomitic-differentiated by the different levels of magnesium carbonate they contain which impart specific qualities to mortar. Historically, calcium lime was used for mortar rather than the dolomitic lime (calcium magnesium carbonate) most often used today. But it is also important to keep in mind the fact that the historic limes, and other components of mortar, varied a great deal because they were natural, as opposed to modern lime which is manufactured and, therefore, standardized. Because some of the kinds of lime, as well as other components of mortar, that were used historically are no longer readily available, even when a conscious effort is made to replicate a "historic" mix, this may not be achievable due to the differences between modem and historic materials.

Lime, itself, when mixed with water into a paste is very plastic and creamy. It will remain workable and soft indefinitely, if stored in a sealed container. Lime (calcium hydroxide) hardens by carbonation absorbing carbon dioxide primarily from the air, converting itself to calcium carbonate. Once a lime and sand mortar is mixed and placed in a wall, it begins the process of carbonation. If lime mortar is left to dry too rapidly, carbonation of the mortar will be reduced, resulting in poor adhesion and poor durability. In addition, lime mortar is slightly water soluble and thus is able to re-seal any hairline cracks that may develop during the life of the mortar. Lime mortar is soft, porous, and changes little in volume during temperature fluctuations thus making it a good choice for historic buildings. Because of these qualities, high calcium lime mortar may be considered for many repointing projects, not just those invalving historic

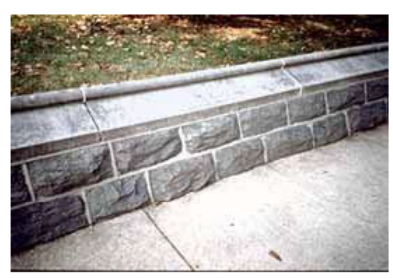
Caulking was inappropriately used here in place of mortar on the top of the wall. As a
not been durable. Photo: NPS files. buildings.

For repointing, lime should conform to ASTM C 207, Type S, or Type SA, Hydrated Lime for Masonry Purposes. This machine-slaked lime is designed to assure high plasticity and water retention. The use of quicklime which must be slaked and soaked by hand may have advantages over hydrated lime in some restoration projects if time and money allow.

\section{Lime Putty}

Lime putty is slaked lime that has a putty or paste-like consistency. It should conform to ASTM C 5. Mortar can be mixed using lime putty according to ASTM C 270 property or proportion specification.

\section{Portland Cement}

More recent, 20th-century mortar has used portland cement as a primary binding material. A straight portland cement and sand mortar is extremely hard, resists the movement of water, shrinks upon setting, and undergoes relatively large thermal movements. When mixed with water, portland cement forms a harsh, stiff paste that is quite unworkable, becoming hard very quickly. (Unlike lime, portland cement will harden regardless of weather conditions and does not require wetting and drying cycles.) Some portland cement assists the workability and plasticity of the mortar without adversely affecting the finished project; it also provides early strength to the mortar and speeds setting. Thus, it may be appropriate to add some portland cement to an essentially lime-based mortar even when repointing relatively soft 18 th or 19th century brick under some circumstances when a slighty harder mortar is required. The more portland cement that is added to a mortar formulation the harder it becomes-and the faster the initial set.

For repointing, portland cement should conform to ASTM C 150. White, non-staining porttand cement may provide a better color match for some historic mortars than the more commonly available grey portland cement. But, it should not be assumed, however, that white portland cement is always appropriate for all historic buildings, since the original mortar may have been mixed with grey cement. The cement should not have more than 0.60 per cent alkali to help avoid efflorescence.

\section{Masonry Cement}

Masonry cement is a preblended mortar mix commonly found at hardware and home repair stores. It is designed to produce mortars with a compressive strength of 750 psi or higher when mixed with sand and water at the job site. It may contain hydrated lime, but it always contains a large amount of portland cement, as well as ground limestone and other workability agents, including air-entraining agents. Because masonry cements are not required to contain hydrated lime, and generally do not contain lime, they produce high strength mortars that can damage historic masonry. For this reason, they generally are not recommended for use on historic masonry buildings.

\section{Lime Mortar (pre-blended)}

Hydrated lime mortars, and pre-blended lime putty mortars with or without a matched sand are commercially available. Custom mortars are also available with color. In most instances, pre-blended lime mortars containing sand may not provide an exact match; however, if the project calls for total repointing, a pre-blended lime mortar may be worth considering as long as the mortar is compatible in strength with the masonry. If the project involves only selected, "spot" repointing, then it may be better to carry out a mortar analysis which can provide a custom pre-blended lime mortar with a matching sand. 
In either case, if a pre-blended lime mortar is to be used, it should contain Type S or SA hydrated lime conforming to ASTM C 207

\section{water}

Water should be potable-clean and free from acids, alkalis, or other dissolved organic materials.

\section{Other Components}

\section{Historic components}

In addition to the color of the sand, the texture of the mortar is of critical importance in duplicating historic mortar. Most mortars dating from the mid-19th century on-with some exceptions-have a fairly homogeneous texture and color. Some earlier mortars are not as uniformly textured and may contain lumps of partially burned lime or "dirty lime", shell (which often provided a source of lime, particularly in coastal areas), natural cements, pieces of clay, lampblack or other pigments, or even animal hair. The visual characteristics of these mortars can be duplicated through the use of similar materials in the repointing mortar.

Replicating such unique or individual mortars will require writing new specifications for each project. If possible, suggested sources for special materials should be included. For example, crushed oyster shells can be obtained in a variety of sizes from poul try supply dealers.

\section{Pigments}

Some historic mortars, particularly in the late 19 th century, were tinted to match or contrast with the brick or stone. Red pigments, sometimes in the form of brick dust, as well as brown, and black pigments were commonly used. Modern pigments are available which can be added to the mortar at the job site, but they should not exceed 10 per cent by weight of the portland cement in the mix, and carbon black should be limited to 2 per cent. only synthetic mineral oxides, which are alkali-proof and sun-fast, should be used to prevent bleaching and fading.

\section{Modern Components}

Admixtures are used to create specific characteristics in mortar, and whether they should be used will depend upon the individual project. Air entraining agents, for example, help the mortar to resist freeze-thaw damage in northern climates. Accelerators are used to reduce mortar freezing prior to setting while retarders help to extend the mortar life in hot climates. Selection of admixtures should be made by the architect or architectural conservator as part of the specifications, not something routinely added by the masons.

Generally, modern chemical additives are unnecessary and may, in fact, have detrimental effects in historic masonry projects. The use of antifreeze compounds is not recommended. They are not very effective with high lime mortars and may introduce salts, which may cause efflorescence later. A better practice is to warm the sand and water, and to protect the completed work from freezing. No definitive study has determined whether air-entraining additives should be used to resist frost action and enhance plasticity, but in areas of extreme exposure requiring high-strength mortars with lower permeability, air-entrainment of 10-16 percent may be desirable (see formula for "severe weather exposure" in Mortar Type and Mix). Bonding agents are not a substitute for proper joint preparation, and they should generally be avoided. If the joint is properly prepared, there will be a good bond between the new mortar and the adjacent surfaces. In addition, a bonding agent is difficult to remove if smeared on a masonry surface.

\section{Mortar Type and Mix}

Mortars for repointing projects, especially those involving historic buildings, typically are custom mixed in order to ensure the proper physical and visual qualities. These materials can be combined in varying proportions to cre ate a mortar with the desired performance and durability. The actual specification of a particular mortar type should take into consideration all of the factors affecting the life of the building including: current site conditions, present condition of the masonry, function of the new mortar, degree of weather exposure, and skill of the mason.

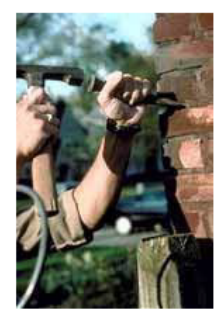

Thus, no two repointing projects are exacty the same. Modern materials specified for use in repointing mortar should conform to specifications of the American Society for Testing and Materials (ASTM) or comparable federal specifications, and the resulting mortar should conform to ASTM C 270, Mortar for Unit Masonry.

Specifying the proportions for the repointing mortar for a specific job is not as difficult as it might seem. Five mortar types, each with a corresponding recommended mix, have been established by ASTM to distinguish high strength mortar from soft flexible mortars. The ASTM designated them in decreasing order of approxima te general strength as Type $M(2,500$ psi), Type $S$ ( 1,800 psi), Type $N$ (750 psi), Type $O$ ( 350 psi) and Type K (75 psi). (The letters identifying the types are from the words MASON WORK using every other letter.) Type K has the highest lime content of the mixes that 
Here, a hammer and chisel are being correctly repointing. Photo: John P. Speweik.

contain portland cement, al though it is seldom used today, except for some historic preservation projects. The designation " $L$ " in the accompanying chart identifies a straight lime and sand mix. Specifying the appropriate ASTM mortar by proportion of ingredients, will ensure the desired physical properties. Unless specified otherwise, measurements or proportions for mortar mixes are always given in the following order: cement-lime-sand. Thus, a Type $K$ mix, for example, would be referred to as $1-3-10$, or 1 part cement to 3 parts lime to 10 parts sand. Other requirements to create the desired visual qualities should be included in the specifications.

The strength of a mortar can vary. If mixed with higher amounts of portland cement, a harder mortar is obtained. The more lime that is added, the softer and more plastic the mortar becomes, increasing its workability. A mortar strong in compressive strength might be desirable for a hard stone (such as granite) pier holding up a bridge deck, whereas a softer, more permeable lime mortar would be preferable for a historic wall of soft brick. Masonry deterioration caused by salt deposition results when the mortar is less permeable than the masonry unit. A strong mortar is still more permeable than hard, dense stone. However, in a wall constructed of soft bricks where the masonry unit itself has a relatively high permeability or vapor transmission rate, a soft, high lime mortar is necessary to retain sufficient permeability.

\section{Budgeting and Scheduling}

Repointing is both expensive and time consuming due to the extent of handwork and special materials required. It is preferable to repoint only those areas that require work rather than an entire wall, as is often specified. But, if 25 to 50 per cent or more of a wall needs to be repointed, repointing the entire wall may be more cost effective than spot repointing.

Total repointing may also be more sensible when access is difficult, requiring the erection of expensive scaffolding (unless the majority of the mortar is sound and unlikely to require replacement in the foreseeable future). Each project requires judgement based on a variety of factors. Recognizing this at the outset will help to prevent many jobs from becoming prohibitively expensive.

In scheduling, seasonal aspects need to be considered first. Generally speaking, wall temperatures between 40 and 95 degrees $F$ ( 8 and 38 degrees $C$ ) will prevent freezing or excessive evaporation of the water in the mortar. Ideally, repointing should be done in shade, away from strong sunlight in order to slow the drying process, especially during hot weather. If necessary, shade can be provided for large-scale projects with appropriate modifications to scaffolding.

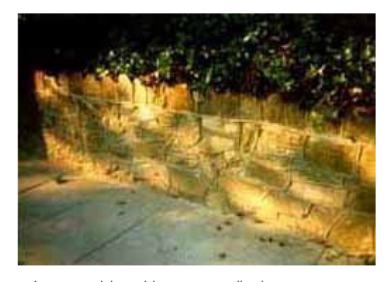

When repairing this stone wall, the mason
matched the raised profile of the original matched the raised profile of the
tuckpointing. Photo: NPS files.

\section{The relationship of repointing to other work proposed on the building must also be}

recognized. For example, if paint removal or cleaning is anticipated, and if the mortar joints are basically sound and need only selective repointing, it is generally better to postpone repointing until after completion of these activities. However, if the mortar has eroded badly, allowing moisture to pene trate deeply into the wall, repointing should be accomplished before cleaning. Related work, such as structural or roof repairs, should be scheduled so that they do not interfere with repointing and so that all work can take maximum advantage of erected scaffolding.

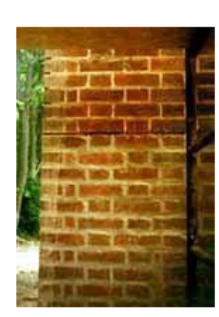

A mechanical grinder improperly used to out at incompatible repointing have seriously damaged the 19 th century brick. Photo: NPS files.
Building managers also must recognize the difficulties that a repointing project can create. The process is time consuming, and scaffolding may need to remain in place for an extended period of time. The joint preparation process can be quite noisy and can generate large quantities of dust which must be controlled, especially at air intakes to protect human health, and also where it might damage operating machinery. Entrances may be blocked from time to time making access difficult for both building tenants and visitors. Clearly, building managers will need to coordinate the repointing work with other events at the site.

\section{Contractor Selection}

Contractor Selection The ideal way to select a contractor is to ask knowledgeable owners of recenty repointed historic buildings for recommendations. Qualified contractors then can provide lists of other repointing projects for inspection. More commonly, however, the contractor for a repointing project is selected through a competitive bidding process over which the client or consultant has only limited control. In this situation it is important to ensure that the specifications stipulate that masons must have a minimum of five years' experience with repointing historic masonry buildings to be eligible to bid on the project. Contracts are aw arded to the lowest responsible bidder, and bidders who have performed poorly on other projects usually can be elimina ted from consideration on this basis, even if they have the lowest prices. 
The contract documents should call for unit prices as well as a base bid. Unit pricing forces the contractor to determine in advance what the cost addition or reduction will be for work which varies from the scope of the base bid. If, for example, the contractor has fifty linear feet less of stone repointing than indicated on the contract documents but thirty linear feet more of brick repointing, it will be easy to determine the final price for the work. Note that each type of work -brick repointing, stone repointing, or similar items-will have its own unit price. The unit price also should reflect quantities; one linear foot of pointing in five different spots will be more expensive than five contiguous linear feet.

\section{Execution of the Work}

\section{Test Panels}

These panels are prepared by the contractor using the same techniques that will be used on the remainder of the project. Several panel locations-preferably not on the front or other highly visible location of the building-may be necessary to include all types of masonry, joint styles, mortar colors, and other problems likely to be encountered on the job.

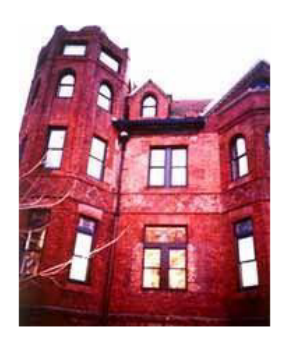

Unskilled repointing has negatively impacted the character of this late-19th century building. Photo: NPS files.

If cleaning tests, for example, are also to be undertaken, they should be carried out in the same location. Usually a 3 foot by 3 foot area is sufficient for brickwork, while a somewhat larger area may be required for stonework. These panels establish an acceptable standard of work and serve as a benchmark for evaluating and accepting subse quent work on the building.

\section{Joint Preparation}

old mortar should be removed to a minimum depth of 2 to 2-1/2 times the width of the joint to ensure an adequate bond and to prevent mortar "popouts." For most brick joints, this will require removal of the mortar to a depth of approximately $\Omega$ to 1 inch; for stone masonry with wide joints, mortar may need to be removed to a depth of several inches. Any loose or disintegrated mortar beyond this minimum depth also should be removed.

Although some damage may be inevitable, careful joint preparation can help limit damage to masonry units. The traditional manner of removing old mortar is through the use of hand chisels and mash hammers. Though labor-intensive, in most instances this method poses the least threat for damage to historic masonry units and produces the best final product.

The most common method of removing mortar, however, is through the use of power saws or grinders. The use of power tools by unskilled masons can be disastrous for historic masonry, particularly soft brick. Using power saws on walls with thin joints, such as most brick walls, almost always will result in damage to the masonry units by breaking the edges and by overcutting on the head, or vertical joints.

However, small pneumatically-powered chisels generally can be used safely and effectively to remove mortar on historic buildings as long as the masons maintain appropriate control over the equipment. Under certain circumstances, thin diamond-bladed grinders may be used to cut out horizontal joints only on hard portland cement mortar common to most early-20th century masonry buildings. Usually, automatic tools most successfully remove old mortar without damaging the masonry units when they are used in combination with hand tools in preparation for repointing. Where horizontal joints are uniform and fairly wide, it may be possible to use a power masonry saw to assist the removal of mortar, such as by cutting along the middle of the joint; final mortar removal from the sides of the joints still should be done with a hand chisel and hammer. Caulking cutters with diamond blades can sometimes be used successfully to cut out joints without damaging the masonry. Caulking cutters are slow; they do not rotate, but vibrate at very high speeds, thus minimizing the possibility of damage to masonry units. Al though mechanical tools may be safely used in limited circumstances to cut out horizontal joints in preparation for repointing, they should never be used on vertical joints because of the danger of slipping and cutting into the brick above or below the vertical joint. Using power tools to remove mortar without damaging the surrounding masonry units also necessitates highly skilled masons experienced in working on historic masonry buildings. Contractors should demonstrate proficiency with power tools before their use is approved.

Using any of these power tools may also be more acceptable on hard stone, such as quartzite or granite, than on terra cotta with its glass-like glaze, or on soft brick or stone. The test panel should determine the acceptability of power tools. If power tools are to be permitted, the contractor should establish a quality control program to account for worker fatigue and similar variables.

Mortar should be removed cleanly from the masonry units, leaving square corners at the back of the cut. Before filling, the joints should be rinsed with a jet of water to remove all loose particles and dust. At the time of filling, the joints should be damp, but with no standing water present. For masonry walls-limestone, sandstone and common brick - that are extremely absorbent, it is recommended that a continual mist of water be applied for a few hours before repointing begins.

Mortar Preparation 
Mortar components should be measured and mixed carefully to assure the uniformity of visual and physical characteristics. Dry ingredients are measured by volume and thoroughly mixed before the addition of any water. Sand must be added in a damp, loose condition to avoid over sanding. Repointing mortar is typically pre-hydrated by adding water so it will just hold together, thus allowing it to stand for a period of time before the final water is added. Half the water should be added, followed by mixing for approximately 5 minutes. The remaining water should then be added in small portions until a mortar of the desired consistency is reached. The total volume of water necessary may vary from batch to batch, depending on weather conditions. It is important to keep the water to a minimum for two reasons: first, a drier mortar is cleaner to work with, and it can be compacted tightly into the joints; second, with no excess water to evaporate, the mortar cures without shrinkage cracks. Mortar should be used within approximately 30 minutes of final mixing, and "retempering, or adding

more water, should not be permitted.

\section{Using Lime Putty to Make Mortar}

Mortar made with lime putty and sand, sometimes referred to as roughage or course stuff, should be measured by volume, and may require slightly different proportions from those used with hydrated lime. No additional water is usually needed to achieve a workable consistency because enough water is already contained in the putty. Sand is proportioned first, followed by the lime putty, then mixed for five minutes or until all the sand is thoroughly coated with the lime putty. But mixing, in the familiar sense of turning over with a hoe, sometimes may not be sufficient if the best possible performance is to be obtained from a lime putty mortar. Although the old practice of chopping, beating and ramming the mortar has largely been forgotten, recent field work has confirmed that lime putty and sand rammed and beaten with a wooden mallet or ax handle, interspersed by chopping with a hoe, can significantly improve workability and performance. The intensity of this action increases the overall lime/sand contact and removes any surplus water by compacting the other ingredients. It may also be advantageous for larger projects to use a mortar pan mill for mixing. Mortar pan mills which have a long tradition in Europe produce a superior lime putty mortar not attainable with today's modern paddle and drum type mixers.

For larger repointing projects the lime putty and sand can be mixed together ahead of time and stored indefinitely, on or off site, which eliminates the need for piles of sand on the job site. This mixture, which resembles damp brown sugar, must be protected from the air in sealed containers with a wet piece of burlap over the top or sealed in a large plastic bag to prevent evaporation and premature carbonation. The lime putty and sand mixture can be recombined into a workable plastic state months later with no additional water.

If portland cement is specified in a lime putty and sand mortar-Type $O(1: 2: 9)$ or Type $K(1: 3: 11)$-the portland cement should first be mixed into a slurry paste before adding it to the lime putty and sand. Not only will this ensure that the portland cement is evenly distributed throughout the mixture, but if dry portland cement is added to wet ingredients it tends to "ball up," jeopardizing dispersion. (Usually water must be added to the lime putty and sand anyway once the portland cement is introduced.) Any color pigments should be added at this stage and mixed for a full five minutes. The mortar should be used within 30 minutes to $1 \Omega$ hours and it should not be retempered. Once portland cement has been added the mortarcan no longer be stored.

\section{Filling the Joint}

Where existing mortar has been removed to a depth of greater than 1 inch, these deeper areas should be filled first, compacting the new mortar in several layers. The back of the entire joint should be filled successively by applying approximately $1 / 4$ inch of mortar, packing it well into the back corners. This application may extend along the wall for several feet. As soon as the mortar has reached thumb-print hardness, another $1 / 4$ inch layer of mortar-approximately the same thickness-may be applied. Several layers will be needed to fill the joint flush with the outer surface of the masonry. It is important to allow each layer time to harden before the next layer is applied; most of the mortar shrinkage occurs during the hardening process and layering thus minimizes overall shrinkage.

When the final layer of mortar is thumb-print hard, the joint should be tooled to match the historic joint. Proper timing of the tooling is important for uniform color and appearance. If tooled when too soft, the color will be lighter than expected, and hairline cracks may occur; if tooled when too hard, there may be dark streaks called "tool burning," and good closure of the mortar against the masonry units will not be achieved.

If the old bricks or stones have worn, rounded edges, it is best to recess the final mortar slightly from the face of the masonry. This treatment will help avoid a joint which is visually wider than the actual joint; it also will avoid creation of a large, thin featheredge which is easily damaged, thus admitting water. After tooling, excess mortar can be removed from the edge of the joint by brushing with a natural bristle or nylon brush. Metal bristle brushes should never be used on historic masonry

\section{Curing Conditions}

The preliminary hardening of high-lime content mortars-those mortars that contain more lime by volume than portland cement, i.e., Type $O(1: 2: 9)$, Type K (1:3:11), and straight lime/sand, Type "L" (0:1:3)-takes place fairly rapidly as water 
in the mix is lost to the porous surface of the masonry and through evaporation. A high lime mortar (especially Type "L") left to dry out too rapidly can result in chalking, poor adhesion, and poor durability. Periodic wetting of the repointed area after the mortar joints are thumb-print hard and have been finish tooled may significantly accelerate the carbonation process. When feasible, misting using a hand sprayer with a fine nozzle can be simple to do for a day or two after repointing. Local conditions will dicta te the frequency of wetting, but initially it may be as often as every hour and gradually reduced to every three or four hours. Walls should be covered with burlap for the first three days after repointing. (Plastic may be used, but it should be tented out and not placed directly against the wall.) This helps keep the walls damp and protects them from direct sunlight. Once carbonation of the lime has begun, it will continue for many years and the lime will gain strength as it reverts back to calcium carbonate within the wall.

\section{Aging the Mortar}

Even with the best efforts at matching the existing mortar color texture, and materials, there will usually be a visible difference between the old and new work, partly because the new mortar has been matched to the unweathered portions of the historic mortar. Another reason for a slight mismatch may be that the sand is more exposed in old mortar due to the slight erosion of the lime or cement. Although spot repointing is generally preferable and some color difference should be acceptable, if the difference between old and new mortar is too extreme, it may be advisable in some instances to repoint an entire area of a wall, or an entire feature such as a bay, to minimize the

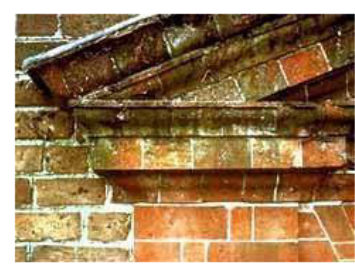

This 18 th century pediment and surrounding wall exhibit distindively different mortar joints. Photo: NPS files. difference between the old and the new mortar. If the mortars have been properly matched, usually the best way to deal with surface color differences is to let the mortars age naturally. Other treatments to overcome these differences, including cleaning the non-repointed areas or staining the new mortar, should be carefully tested prior to implementation.

Staining the new mortar to achieve a better color match is generally not recommended, but it may be appropriate in some instances. Athough staining may provide an initial match, the old and new mortars may weather at different rates, leading to visual differences after a few seasons. In addition, the mixtures used to stain the mortar may be harmful to the masonry; for example, they may introduce salts into the masonry which can lead to efflorescence.

\section{Cleaning the Repointed Masonry}

If repointing work is carefully executed, there will be little need for cleaning other than to remove the small amount of mortar from the edge of the joint following tooling. This can be done with a stiff natural bristle or nylon brush after the mortar has dried, butbefore it is initially set (1-2 hours). Mortar that has hardened can usually be removed with a wooden paddle or, if necessary, a chisel.

Further cleaning is best accomplished with plain water and natural bristle or nylon brushes. If chemicals must be used, they should be selected with extreme caution. Improper cleaning can lead to deterioration of the masonry units, deterioration of the mortar, mortar smear, and efflorescence. New mortar joints are especially susceptible to damage because they do not become fully cured for several months. Chemical cleaners, particularly acids, should never be used on dry masonry. The masonry should always be completely soaked once with water before chemicals are applied. After cleaning, the walls should be flushed again with plain water to remove all traces of the chemicals.

Several precautions should be taken if a freshly repointed masonry wall is to be cleaned. First, the mortar should be fully hardened before cleaning. Thirty days is usually sufficient, depending on weather and exposure; as mentioned previously, the mortar will continue to cure even after it has hardened. Test panels should be prepared to evaluate the effects of different cleaning methods. Generally, on newly repointed masonry walls, only very low pressure (100 psi) water washing supplemented by stiff natural bristle or nylon brushes should be used, except on glazed or polished surfaces, where only soft cloths should be used.***

New construction "bloom" or efflorescence occasionally appears within the first few months of repointing and usually disappears through the normal process of weathering. If the efflorescence is not removed by natural processes, the safest way to remove it is by dry brushing with stiff natural or nylon bristle brushes followed by wet brushing. Hydrochloric (muriatic) acid, is generally ineffective, and it should not be used to remove efflorescence. It may libera te additional salts, which, in turn, can lead to more efflorescence.

Surface grouting is sometimes suggested as an al ternative to repointing brick buildings, in particular. This process involves the application of a thin coat of cement-based grout to the mortar joints and the mortar/brick interface. To be effective, the grout must extend slighty onto the face of the masonry units, thus widening the joint visually. The change in the joint appearance can alter the historic character of the structure to an unacceptable degree. In addition, although 
masking of the bricks is intended to keep the grout off the remainder of the face of the bricks, some level of residue, called "veiling," will inevitably remain. Surface grouting cannot substitute for the more extensive work of repointing, and it is not a recommended treatment for historic masonry.

**Additional information on masonry cleaning is presented in Preservation Briefs 1: Assessing Cleaning and WaterRepellent Treatments for Historic Masonry Buildings, Robert C. Mack, FAIA, and Anne E. Grimmer, Washington, D.C.: Technical Preservation Services, National Park Service, U.S. Department of the Interior, 2000; and Keeping it Clean: Removing Exterior Dirt, Paint, Stains \& Graffiti from Historic Masonry Buildings, Anne E. Grimmer, Washington, D.C.: Technical Preservation Services, National Park Service, U.S. Department of the Interior, 1988

\section{Visually Examining the Mortar and the Masonry Units}

A simple in situ comparison will help determine the hardness and condition of the mortar and the masonry units. Begin by scraping the mortar with a screwdriver, and gradually tapping harder with a cold chisel and mason's hammer. Masonry units can be tested in the same way beginning, even more gently, by scraping with a fingernail. This relative analysis which is derived from the 10-point hardness scale used to describe minerals, provides a good starting point for selection of an appropriate mortar. It is described more fully in "The Russack System for Brick \& Mortar Description" referenced in reading List at the end of this Brief.

Mortar samples should be chosen carefully, and picked from a variety of locations on the building to find unweathered mortar, if possible. Portions of the building may have been repointed in the past while other areas may be subject to conditions causing unusual deterioration. There may be several colors of mortar dating from different construction periods or sand used from different sources during the initial construction. Any of these situations can give false readings to the visual or physical characteristics required for the new mortar. Variations should be noted which may require developing more than one mix.

1. Remove with a chisel and hammer three or four unweathered samples of the mortar to be matched from several locations on the building. (Set the largest sample aside--this will be used la ter for comparison with the repointing mortar). Removing a full representation of samples will allow selection of a "mean" or average mortar sample.

2. Mash the remaining samples with a wooden mallet, or hammer if necessary, until they are separated into their constituent parts. There should be a good handful of the material.

3. Examine the powdered portion - the lime and/or cement matrix of the mortar. Most particularly, note the color. There is a tendency to think of historic mortars as having white binders, but grey portland cement was available by the last quarter of the 19 th century, and traditional limes were also sometimes grey. Thus, in some instances, the natural color of the historic binder may be grey, rather than white. The mortar may also have been tinted to create a colored mortar, and this color should be identified at this point.

4. Carefully blow away the powdery material (the lime and/or cement matrix which bound the mortar together).

5. With a low power (10 power) magnifying glass, examine the remaining sand and other materials such as lumps of lime or shell.

6. Note and record the wide range of color as well as the varying sizes of the individual grains of sand, impurities, or other materials.

\section{Other Factors to Consider}

Color

Regardless of the color of the binder or colored additives, the sand is the primary material that gives mortar its color. A surprising variety of colors of sand may be found in a single sample of historic mortar, and the different sizes of the grains of sand or other materials, such as incompletely ground lime or cement, play an important role in the texture of the repointing mortar. Therefore, when specifying sand for repointing mortar, it may be necessary to obtain sand from several sources and to combine or screen them in order to approximate the range of sand colors and grain sizes in the historic mortar sample.

\section{Pointing Style}

Close examination of the historic masonry wall and the techniques used in the original construction will assist in maintaining the visual qualities of the building. Pointing styles and the methods of producing them should be examined. It is important to look at both the horizontal and the vertical joints to determine the order in which they were tooled and whether they were the same style. Some late-19th and early-20th century buildings, for example, have horizontal joints that were raked back while the vertical joints were finished flush and stained to match the bricks, thus creating the illusion of horizontal 
bands. Pointing styles may also differ from one facade to another; front walls often received greater attention to mortar detailing than side and rear walls. Tuckpointing is not true repointing but the application of a raised joint or lime putty joint on top of flush mortar joints. Penciling is a purely decorative, painted surface treatment over a mortar joint, often in a contrasting color.

Masonry Units

The masonry units should also be examined so that any replacement units will match the historic masonry. Within a wall there may be a wide range of colors, textures, and sizes, particularly with hand-made brick or rough-cut, locally-quarried stone. Replacement units should blend in with the full range of masonry units rather than a single brick or stone.

Matching Color and Texture of the Repointing Mortar

New mortar should match the unweathered interior portions of the historic mortar. The simplest way to check the match is to make a small sample of the proposed $\mathrm{mix}$ and allow it to cure at a temperature of approximately 70 degrees $F$ for about a week, or it can be baked in an oven to speed up the curing; this sample is then broken open and the surface is compared with the surface of the largest "saved" sample of historic mortar.

If a proper color match cannot be achieved through the use of natural sand or colored aggregates like crushed marble or brick dust, it may be necessary to use a modern mortar pigment.

During the early stages of the project, it should be determined how closely the new mortar should match the historic mortar. Will "quite close" be sufficient, or is "exactly" expected? The specifications should state this clearly so that the contractor has a reasonable idea how much time and expense will be required to develop an acceptable match.

The same judgment will be necessary in matching replacement terra cotta, stone or brick. If there is a known source for replacements, this should be included in the specifications. If a source cannot be determined prior to the bidding process, the specifications should include an estimated price for the replacement materials with the final price based on the actual cost to the contractor.

Mortar Types (Measured by volume)

\begin{tabular}{llll}
\hline Designation & Cement & Hydrated Lime or Lime Putty & Sand \\
\hline M & 1 & $1 / 4$ & $3-33 / 4$ \\
\hline$S$ & 1 & $1 / 2$ & $4-41 / 2$ \\
\hline$N$ & 1 & 1 & $5-6$ \\
\hline$O$ & 1 & 2 & $8-9$ \\
\hline$K$ & 1 & 3 & $10-12$ \\
\hline "L" & 0 & 1 & $21 / 4-3$ \\
\hline
\end{tabular}

Suggested Mortar Types for Differen $t$ Ex posures

\begin{tabular}{llll}
\hline & Exposure & & \\
\hline Masonry Material & Sheltered & Moderate & Severe \\
\hline Very durable: granite, hard-cored brick, etc. & $\mathrm{O}$ & $\mathrm{N}$ & $\mathrm{S}$ \\
\hline Moderately durable:limestone, durable stone, molded brick & $\mathrm{K}$ & $\mathrm{O}$ & $\mathrm{N}$ \\
\hline Minimally durable:soft hand-made brick & "L" & $\mathrm{K}$ & $\mathrm{O}$ \\
\hline
\end{tabular}

\section{Summary and References}

\section{For the Owner/Administrator}

The owner or administrator of a historic building should remember that repointing is likely to be a lengthy and expensive process. First, there must be adequate time for evaluation of the building and investigation into the cause of problems. Then, there will be time needed for preparation of the contract documents. The work itself is precise, time-consuming and noisy, and scaffolding may cover the face of the building for some time. Therefore, the owner must carefully plan the work 
to avoid problems. Schedules for both repointing and other activities will thus require careful coordination to avoid unanticipated conflicts. The owner must avoid the tendency to rush the work or cut corners if the historic building is to retain its visual integrity and the job is to be durable.

\section{For the Architect/Consultant}

Because the primary role of the consultant is to ensure the life of the building, a knowledge of historic construction techniques and the special problems found in older buildings is essential. The consultant must assist the owner in planning for logistical problems relating to research and construction. It is the consultant's responsibility to determine the cause of the mortar deterioration and ensure that it is corrected before the masonry is repointed. The consultant must also be prepared to spend more time in project inspections than is customary in modernconstruction.

\section{For the Masons}

Successful repointing depends on the masons themselves. Experienced masons understand the special requirements for work on historic buildings and the added time and expense they require. The entire masonry crew must be willing and able to perform the work in conformance with the specifications, even when the specifications may not be in conformance with standard practice. At the same time, the masons should not hesitate to question the specifications if it appears that the work specified would damage the building.

\section{Conclusion}

A good repointing job is meant to last, at least 30 years, and preferably $50-100$ years. Shortcuts and poor craftsmanship result not only in diminishing the historic character of a building, but also in a job that looks bad, and will require futur e repointing sooner than if the work had been done correctly. The mortar joint in a historic masonry building has often been called a wall's "first line of defense." Good repointing practices guarantee the long life of the mortar joint, the wall, and the historic structure. Although careful maintenance will help preserve the freshly repointed mortar joints, it is important to remember that mortar joints are intended to be sacrificial and will probably require repointing sometime in the future. Nevertheless, if the historic mortar joints proved durable for many years, then careful repointing should have an equally long life, ultimately contributing to the preservation of the entire building.

\section{Useful Addresses}

Brick Institute of America

11490 Commerce Park Drive

Reston, VA 22091

National Lime Association

200 N. Glebe Road, Suite 800

Arlington, VA 22203

Portland Cement Association

5420 Old Orchard Road

Skokie, IL 60077

\section{Acknowledgments}

Robert C. Mack, FAIA, is a principal in the firm of MacDonald \& Mack, Architects, Ltd, , an architectural firm that specializes in historic buildings in Minneapolis, Minnesota. John P. Speweik, CSI, Toledo, Ohio, is a 5th-generation stonemason, and principal in U.S. Heritage Group, Inc., Chicago, Illinois, which does custom historic mortar matching. An ne E. Grimmer, Senior Architectural Historian, National Park Service, was responsible for developing and coordinating the revision of this Preservation Brief $f_{f}$ incorporating professional comments, and the technical editing.

The authors and the editor wish to thank the following for the professional and technical review they provided: Mark Macpherson and Ron Peterson, Masonry Restoration Contractors, Macpherson-Towne Company, Minneapolis, MN; Lorraine Schnabel, Architectural Conservator, John Milner Associates, Inc., Philadelphia, PA; Lauren B. Sickels-Taves, Ph.D., Architectural Conservator, Biohistory International, Huntington Woods, MI; and the following National Park Service professional staff, including: E. Blaine Cliver, Chief, Historic American Buildings Survey/Historic American Engineering Record; Douglas C. Hicks, Deputy Superintendent, Historic Preservation Training Center, Frederick, MD; Chris McGuigan, Supervisory Exhibits Specialist, Historic Preservation Training Center, Frederick, MD; Charles E. Fisher, Sharon C. Park,

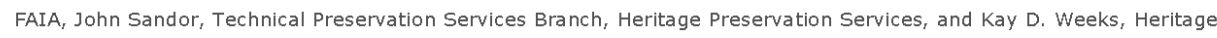
Preservation Services.

The original version of this brief, Repointing Mortar Joints in Historic Brick Buildings, was written by Robert C. Mack in 1976, and was revised and updated in 1980 by Robert C. Mack, de Teel Patterson Tiller, and James S. Askins. 
This publication has been prepared pursuant to the National Historic Preservation Act of 1966, as amended, which directs the Secretary of the Interior to develop and make available information concerning historic properties. Technical Preservation Services (TPS), National Park Service prepares standards, guidelines, and other educational materials on responsible historic preservation treatments for a broad public.

October 1998

\section{Reading List}

Ashurst, John \& Nicola. Practical Building Conservation. Vol. 3: Mortars, Plasters and Renders. New York: Halsted Press, a Division of John Wiley \& Sons, Inc., 1988.

Cliver, E. Blaine. "Tests for the Analysis of Mortar Samples." Bulletin of the Association for Preservation Technology. Vol. 6, No. 1 (1974), pp. 68-73.

Coney, William B., AIA. Masonry Repointing of Twentieth-Century Buildings. Illinois Preservation Series. Number 10. Springfield, IL: Division of Preservation Services, Illinois Historic Preservation Agency, 1989.

Davidson, J.I. "Masonry Mortar." Canadian Building Digest. CBD 163. Ottawa, ONT: Division of Building Research, National Research Council of Canada, 1974.

Ferro, Maximillian L., AIA, RIBA. "The Russack System for Brick and Mortar Description: A Fleld Method for Assessing Masonry Hardness." Technology and Conservation. Vol. 5, No. 2 (Summer 1980), pp. 32-35.

Hooker, Kenneth A. "Field Notes on Repointing." Aberdeen's Magazine of Masonry Construction. Vol. 4, No. 8 (August 1991), pp. 326-328.

Jedrzejewska, H. "Old Mortars in Poland: A New Method of Investigation." Studies in Conservation. Vol. 5, No. 4 (1960), pp. 132-138.

"Lime's Role in Mortar." Aberdeen's Magazine of Masonry Construction. Vol. 9, No. 8 (August 1996), pp. 364-368.

Phillips, Morgan W. "Brief Notes on the Subjects of Analyzing Paints and Mortars and the Recording of Moulding Profiles: The Trouble with Paint and Mortar Analysis." Bulletin of the Association for Preservation Technology. Vol. 10, No. 2 (1978), pp. 77-89.

Preparation and Use of Lime Mortars: An Introduction to the Principles of Using Lime Mortars. Scottish Lime Centre for Historic Scotland. Edinburgh: Historic Scotland, 1995.

Schierhorn, Carolyn. "Ensuring Mortar Color Consistency." Aberdeen's Magazine of Masonry Construction. Vol. 9 No. 1 (January 1996), pp. 33-35.

"Should Air-Entrained Mortars Be Used?" Aberdeen's Magazine of Masonry Construction. Vol. 7, No. 9 (September 1994), pp. 419-422.

Sickels-Taves, Lauren B. "Creep, Shrinkage, and Mortars in Historic Preservation." Journal of Testing and

Evaluation, JTEVA. Vol. 23, No. 6 (November 1995), pp. 447-452.

Speweik, John P. The History of Masonry Mortar in America, 1720-1995. Arlington, VA: National Lime Association, 1995.

Speweik, John P. "Repointing Right: Why Using Modern Mortar Can Damage a Historic House." Old-House Journal. Vol. XXV, No. 4 (July-August 1997), pp. 46-51.

Technical Notes on Brick Construction. Brick Institute of America, Reston, VA.

"Moisture Resistance of Brick Masonry: Maintenance." 7F. February 1986.

"Mortars for Brick Masonry." 8 Revised II. November 1989.

"Standard Specification for Portland Cement-Lime Mortar for Brick Masonry." 8A Revised. September 1988.

"Mortar for Brick Masonry-Selection and Controls." 8B Reissued. September 1988. (July/August 1976).

"Guide Specifications for Brick Masonry, Part V Mortar and Grout." 11E Revised. September 1991.

"Bonds and Patterns in Brickwork." 30 Reissued. September 1988. 


\title{
11.2.2 Guidelines for evaluating the condition of brick masonry and mortar, 352016 (GSA.gov).
}

\section{Guidelines forEvaluating theCondition of Brick Masonry and Mortar}

\author{
Guidelines for Evaluating the Condition of Brick Masonry and Mortar \\ CAUTION: Thismethod of conditionassessmentis destructiveand should only beused to testareas believed to be \\ deteriorated. This test should be performed only by an experienced mason. \\ This method of evaluation was developed byrestoration architectMax Ferroand masonry conservatorTom \\ Russackand appeared in the January/February 1987 issue of the Old House Journal. \\ Materials \\ - Mason's hammer \\ - Cold chisel (1/2 to $1-1 / 2$ inches) \\ - Sturdy slotted screw driver \\ The deterioration of brickand mortar are evaluated by rating each on a scale from 0 to 10 based on their level hardness or \\ softness. A rating of 0 indicates severe brick and/or mortar deterioration. A brief description of each rating follows. \\ Assessment of Brick \\ - A rating of ' 4 ' or below indicates brick in an unsalvageable condition. \\ - A rating between ' 5 ' and ' 7 ' indicates that some remedial measures may need to be taken. \\ - A rating of ' 10 ' indicates that the brick units are in good, sound condition.

Rating Description \\ Bricks are totally disintegrated. \\ $1 \quad$ Evidence of spalling at least 1/4" to 3" deep.
}

35 Retrieved from: https://www.gsa.gov/real-estate/historic-preservation/historic-preservation-policytools/preservation-tools-resources/technical-documents. 


\begin{tabular}{|c|c|}
\hline 2 & $\begin{array}{l}\text { Slighterosion at corners of brick; slightpowdering of surface when rubbed with hand or scraped } \\
\text { with fingernail. }\end{array}$ \\
\hline 3 & Spalling brick in layers when rubbed with hand; fragments do not powder. \\
\hline 4 & $\begin{array}{l}\text { Brickscan bebroken by poking and jabbing with screwdriver; fragments are semi-hard and } \\
\text { resemble compacted clay. }\end{array}$ \\
\hline 5 & $\begin{array}{l}\text { FIRST CLASS OFSTABLE, STRUCTURALLY SOUND BRICK: Screwdriver can penetrate the brick by hand } \\
\text { roughly } 1 / 4 " \text { but brick does not crumble. }\end{array}$ \\
\hline 6 & $\begin{array}{l}\text { Screwdrivercan penetratethebrickroughly } 1 / 4 " \text { ", butONLY with the assistance of a hammer; this } \\
\text { may cause coarse jagged piecesto become dislod ged. }\end{array}$ \\
\hline \multirow[t]{2}{*}{7} & $\begin{array}{l}\text { Screwdriver is unable to penetratethe brickeven with assistance from hammer but may make a } \\
\text { slight impression in the surface. There may be a slightring or bounce as the screwd river hits the }\end{array}$ \\
\hline & surface. \\
\hline 8 & Chisel is necessary to crack the brick. \\
\hline 9 & $\begin{array}{l}\text { Chise is unable to make an ind entation or impression in the brick; brick shears cleanly; brick isstrong } \\
\text { with crisp edges and corners. }\end{array}$ \\
\hline
\end{tabular}

\section{Assessment of Mortar}

- A rating between ' 0 ' and ' 4 ' indicates that repointing is necessary.

- A rating between ' 5 ' and ' 8 ' indicates mortar in satisfactory condition.

- A rating of ' 9 ' or ' 10 ', ind icates that the mortar is too hard and should be replaced with a softer mortar.

\begin{tabular}{ll}
\hline Rating & Description \\
\hline 0 & No evidence of mortar within at least 1-1/2" of the wall face. \\
\hline 1 & Mortar crumb leswhen poked with finger or screwd river; many surface irregularities are evident. \\
\hline 3 & Mortar is easily removed with screwd river, but FEW surface irregularities are evident injoint. \\
\hline 4 & $\begin{array}{l}\text { Mortarcollapsesand freely and cleanly breaksadhesion with brickwhen scored along centerline } \\
\text { with screwdriver. }\end{array}$ \\
\hline 5 & Slightspalling occursatedgesand corners of brickwhen mortar is scored and tapped with screwdriver.. \\
\hline 6 & $\begin{array}{l}\text { Screwdriver is unable to dislodgethe mortar; chiselcan disengage and pop mortar free } \\
\text { without damaging the brick }\end{array}$ \\
\hline 7 & $\begin{array}{l}\text { Edges and corners of brick are slightly marred when mortar is scored with a chisel. } \\
\text { to the brick. }\end{array}$ \\
\hline 8 & $\begin{array}{l}\text { Severalblowswith hammer and chisel are required to breakthe mortar into several large pieces; } \\
\text { bricks will be noticeably marred. }\end{array}$ \\
\hline 9 & MORTAR IS STRONGERTHANTHEBRICKS: Successiveblowswith hammerand chisel crackbrick
\end{tabular}

MORTAR IS STRONGERTHANTHE BRICKS: Successive blowswith hammerand chisel crack brick. 


MORTARHAS HIGH PORTLAND CEMENT CONTENT: Successive blows with hammer and chisel pulverizes
the brickwork.
Procedure code:
421109G
Source:
NPS Southeast Regional Office
Division:
Masonry
Section:
Brick Unit Masonry
Last Modified:
08/17/2016

\subsubsection{Removing and replacing deteriorated brick masonry, ${ }^{36} 2018$} (GSA.gov).

\section{Removing and Replacing Deteriorated Brick Masonry}

\section{PART 1---GENERAL}

\subsection{SUMMARY}

A. This procedure includes guidance on removing and replacing deteriorated brick masonry. It should be used inconjunction with the procedure on repointing historic masonry. For guidance on repointing, see 04520-02-R"Repointing Masonry Using Lime Mortar".

B. See 01100-07-Sfor general project guidelines to be reviewed along with this procedure. Theseguidelines cover the following sections:

1. Safety Precautions

2. Historic Structures Precautions

3. Submittals

4. Quality Assurance

5. Delivery, Storage and Handling

6. Project/Site Conditions

7. Sequencing and Scheduling

8. General Protection (Surface and Surrounding) Theseguidelines should be reviewed prior to performing this procedure and should be followed, when applicable, along with recommendations from the Regional Historic Preservation Officer (RHPO).

C. For general information on the characteristics, uses and problems associated with brick, see 04211-08-S.

36 Retrieved from: https://www.gsa.gov/real-estate/historic-preservation/historic-preservation-policytools/preservation-tools-resources/technical-documents. 


\subsection{PROJECT/SITE CONDITIONS}

A. Environmental Requirements:

1. Do not proceed with brick replacement under adverse weather conditions, or when temperatures a re below or above manufacturer's recommended limitations for installation; Proceed with the work only when forecasted weather conditions are favorable for proper cure.

2. Wet Weather: Do not apply or mix mortar on outside surfaces with standing water or outside during rain.

3. Cold Weather, winter construction is not allowed without consent of RHPO; Winter construction when surface temperature of masonry is below $400 \mathrm{~F}$. or air temperature is predicted to be below $400 \mathrm{~F}$. within 48 hours. See \#6 below.

4. Hot Weather: The surface temperature of the work, not the ambient temperature, should not be higher than $1000 \mathrm{~F}$.; Mortar mixing should be done only in the shade; Cover mortar with water-misted burlap in hot weather to reduce evaporation; Pointing work should be done in the shade; Work around the building during the day so that the fresh work will be shielded from direct sunlight to reduce evaporation rate. High temperatures can ca use flash setting of cements and rapid evaporation of water in the mix, leading to lack of development of final strength by the cement.

5. All materials must be kept above 40degrees F.

6. Special Precautions and Notes: Do not allow masonry to freeze until mortar is thoroughly dry and hardening almost complete (approx. three days time); The setting of lime mortar is very much slower than that of cement mortar because the curing requires the absorption of carbon dioxide to form hard lime carbonates; It is a very lengthy process, so do not expect it to become hard immediately, especially at the core of large masses of masonry.

\section{PART 2---PRODUCTS}

\subsection{MATERIALS}

A. Salvaged Brick: Approved by RHPO, sound, crack free, clean brick without face chips larger than $1 / 2$ inch, salvaged from removal of removed face brick work of same type.

B. Replacement Brick: Approved by RHPO.

C. Brick slips: Approved by RHPO.

D. Mortar to match existing (see 04100-03-S "Preparing Lime Mortar for Repointing Masonry")

\subsection{EQUIPMENT}

A. Trowel

B. Joint tools

c. Chisel

D. Hawk

E. Hammer

F. Stiff bristle brushes

\section{PART 3---EXECUTION}




\subsection{EXAMINATION}

A. Deterioration of brick due to moisture is evident as spalling, erosion, cracking, peeling paint, and deteriorated mortar joints.

B. Some causes of brick deterioration include:

1. Rising damp,

2. the accumulation of dissolved acids carried by rainwater and condensation,

3. soluble salts crystallizing in the pores of the brick face,

4. alternate freezing and thawing, and e) the accumulation of dirt and air-borne particles on the exterior surface.

\subsection{PREPARATION}

A. Surface Preparation:

1. Wet brick having absorption rates greater than $0.025 \mathrm{oz}$. per sq. inch per minute.

- On the flat side of a brick, deposit water on an area approximately the size of a 25 cent coin.

- If the water disappears in less than 30 seconds, wet the bricks.

2. Absorptive brick should be thoroughly soaked in the pile each afternoon prior to the day they are to be used.

3. Cover the bricks with tarps or heavy paper to prevent evaporation.

4. Wet brick as necessary during the day; Sprinkle the brick pile with a hose for a period long enough for water to run down the side of the pile; Use wetting methods which ensure that each masonry unit is nearly saturated but surface dry when laid; (DO NOT wet stone masonry units).

5. Repair flashing if necessary.

6. Where fresh masonry joins existing work, clean the exposed surface of the set masonry by removing loose brick and mortar and wet lightly to obtain the best possible bond with the new work.

\subsection{ERECTION, INSTALLATION, APPLICATION}

A. Replacing Deteriorated Masonry with Brickslips: The use of brick slips should be limited to replacement of individual bricks or to small areas of brickwork.

NOTE: Brick slips are brick facings about 1 inch thick. They are used when damage to adjacent sound brickwork is likely to occur if full-size replacement is attempted.

1. Cut out the deteriorated masonry to a regular shape.

2. Clean the cavity of loose mortar and other debris by hand using a chisel and stiff bristle brushes.

3. Solidly set the slip in a bed of mortar.

B. Replacing Deteriorated Units with Full-Size Bricks:

1. Carefully remove deteriorated brick units by hand using a hammer and chisel.

2. Rebuil $\mathbf{d}$ back-up and substrate as required to replace any unsound material that was removed.

3. Clean the cavity of loose mortar and other debris by hand using a chisel and stiff bristle brushes.

4. Lightly wet the exposed brick surfaces.

5. Lay brick units with completely filled bed and head joints; Butter ends with sufficient mortar to fill head joints and shove into place.

NOTE: Lay masonry plumb and true following the coursing and patterns of the adjacent existing sound construction; Level off work at required heights and form beds to build-in salvaged or moved materials.

6. If adjustments are required, remove units, clean off mortar and reset in fresh mortar. 
7. Blend new work into existing work smoothly with no lines of demarcation and no change of pattern or coursing.

8. Rake all joints in replacement work to receive tuck pointing; Joints up to $3 / 8$ " in width shall be raked to a depth of $1 / 2$ "; Joints $3 / 8^{\prime \prime}$ in width shall be raked to a depth of $1 "$.

9. Brush all excess mortar from the wall surface frequentlyduring the work; Protect all existing surfaces from mortar dripping and splashing.

\subsection{ADJUSTING/CLEANING}

A. Clean off adjacent surfaces which have been spattered during the course of the work. Rinse immediately with clean, clear water.

B. Wipe all excess mortar as the work progresses. Dry brush at the end of each day's work.

C.After mortar is thoroughly set and cured, remove loose mortar and dirt from new masonry surfaces.

C. Wash down the masonry surface with clean, clear water.

Procedure code:

$421102 \mathrm{~S}$

Source:

Hspg Prepared For Nps - Sero

Division:

Masonry

Section:

Brick Unit Masonry

Last Modified:

01/25/2017 


\subsubsection{Patching cracks in brick masonry, 372018 (GSA.gov).}

\section{Patching Cracksin Brick Masonry}

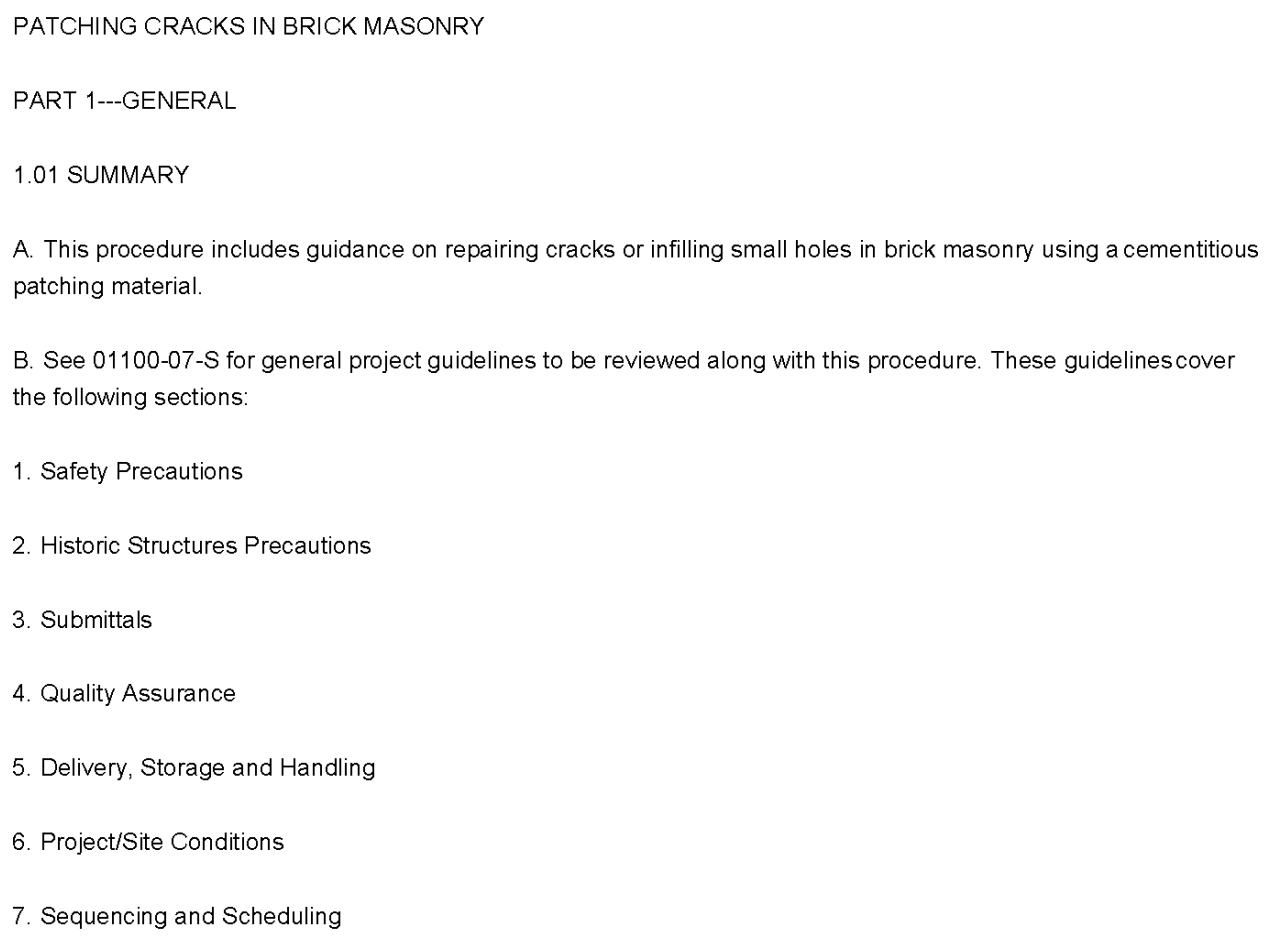

37 Retrieved from: https://www.gsa.gov/real-estate/historic-preservation/historic-preservation-policytools/preservation-tools-resources/technical-documents. 
8. General Protection (Surface and Surrounding)

These guidelines should be reviewed prior to performing this procedure and should be followed, when applicable, along with recommendations from the Regional Historic Preservation Officer (RHPO)

PART 2---PRODUCTS

2.01 MANUFACTURERS

A. Jahn Restoration Techniques and Research (Cathedral Stone Company)

B. Edison Chemical Systems, Inc.

2.02 MATERIALS

A. Cementitious patching material such as "M70 Stone Restoration Mortar" (Jahn Restoration), "Custom System 45" Edison Chemical Systems), or approved equal.

B. Clean, soft cloths

C. Clean, potable water

2.03 EQUIPMENT

A. Stiff natural bristle brushes

B. Trowel

C. Chisel

D. Hammer

E. Putty knife

PART 3---EXECUTION

3.01 ERECTION, INSTALLATION, APPLICATION

A. Remove all loose materials from cracked brickwork.

B. Widen crack to $1 / 8^{\prime \prime}$ wide and grout crack and any voids with a cementitious grout that is color matched to clean brick. The grout should be compatible in texture and porosity to the adjacent masonry. Tape joint to provide a clean neat finished appearance.

C. Severely cracked brick associated with displaced masonry should be dismantled. Probe interior conditions and 
repair following an engineer's evaluation. Rebuild brickwork to match existing bonding patterns and use salvaged brick where possible and new brick to match existing color, texture and porosity.

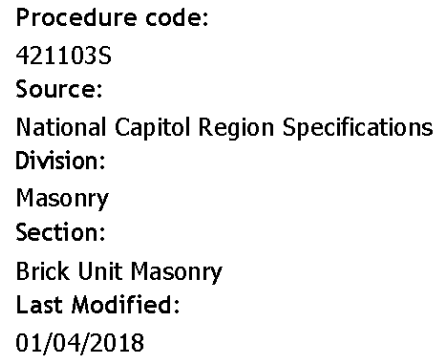




\section{Stage III - Other}

Bldg. 550 has some original features from 1942 that do not have standard treatment measures. These features were standard features used throughout the construction of the WWII buildings at Camp McCoy.

The original siding was asbestos shingle siding with a scalloped bottom edge, measuring approximately 11" x 24" (see Figure 98). This was removed during asbestos abatement in 2019. A comparable type of siding with the faux wood grain and scalloped bottom made with fibre-cement is readily available commercially and should be installed on the exterior walls of Bldg. 550 .

Figure 98. Original asbestos shingle siding with a scalloped bottom edge on the northeast corner of Bldg. 550, 2018.

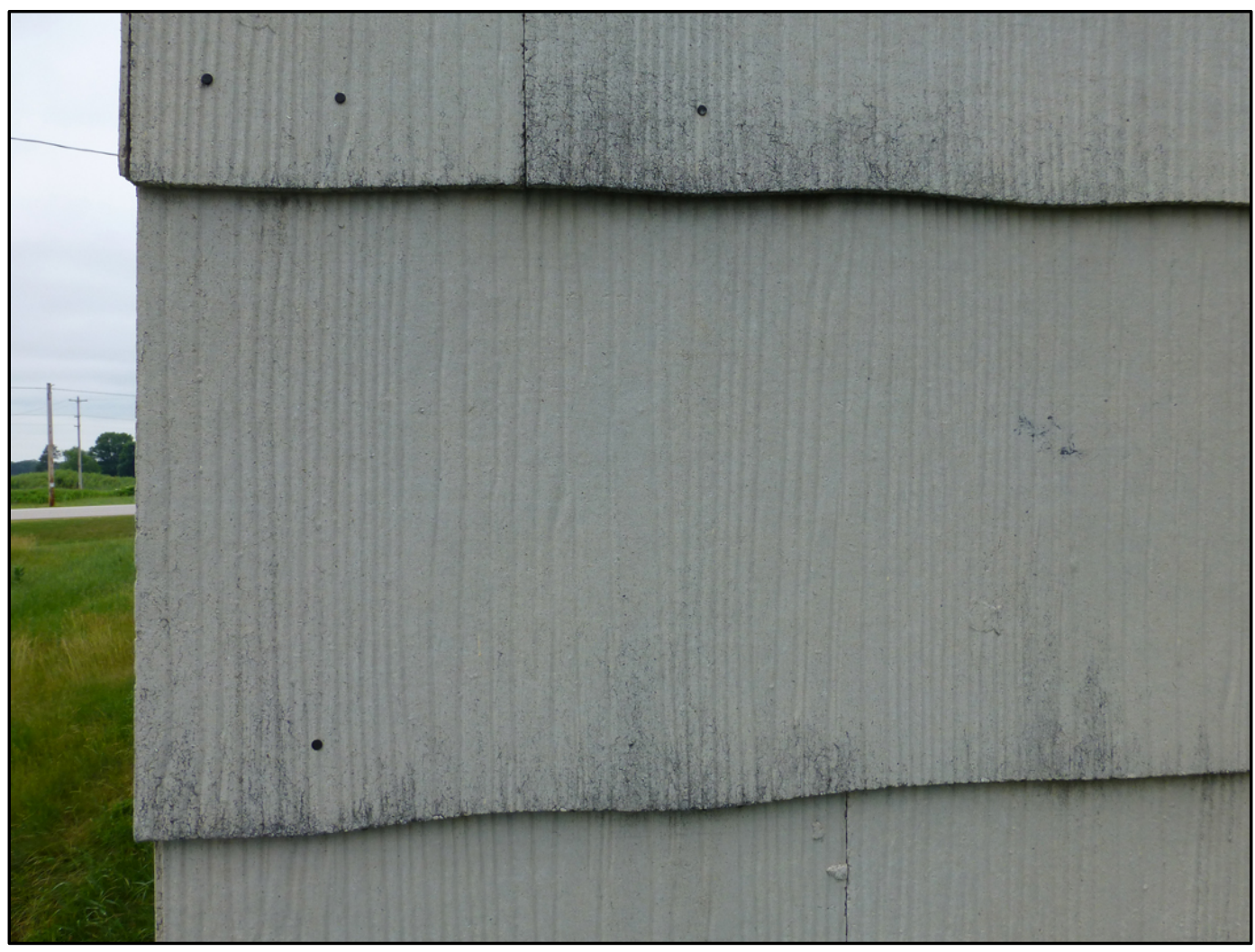

The roof currently is covered with deep red 3-tab asphalt shingles (see Figure 99). It is not known when these shingles were installed. This type of $3^{-}$ tab asphalt roofing shingles are still available in the market. When replacement of shingles individually or wholesale is needed, the replacement should be in-kind to these red three-tab shingles. 
Figure 99. Red three-tab asphalt shingles on Bldg. 550, 2018.

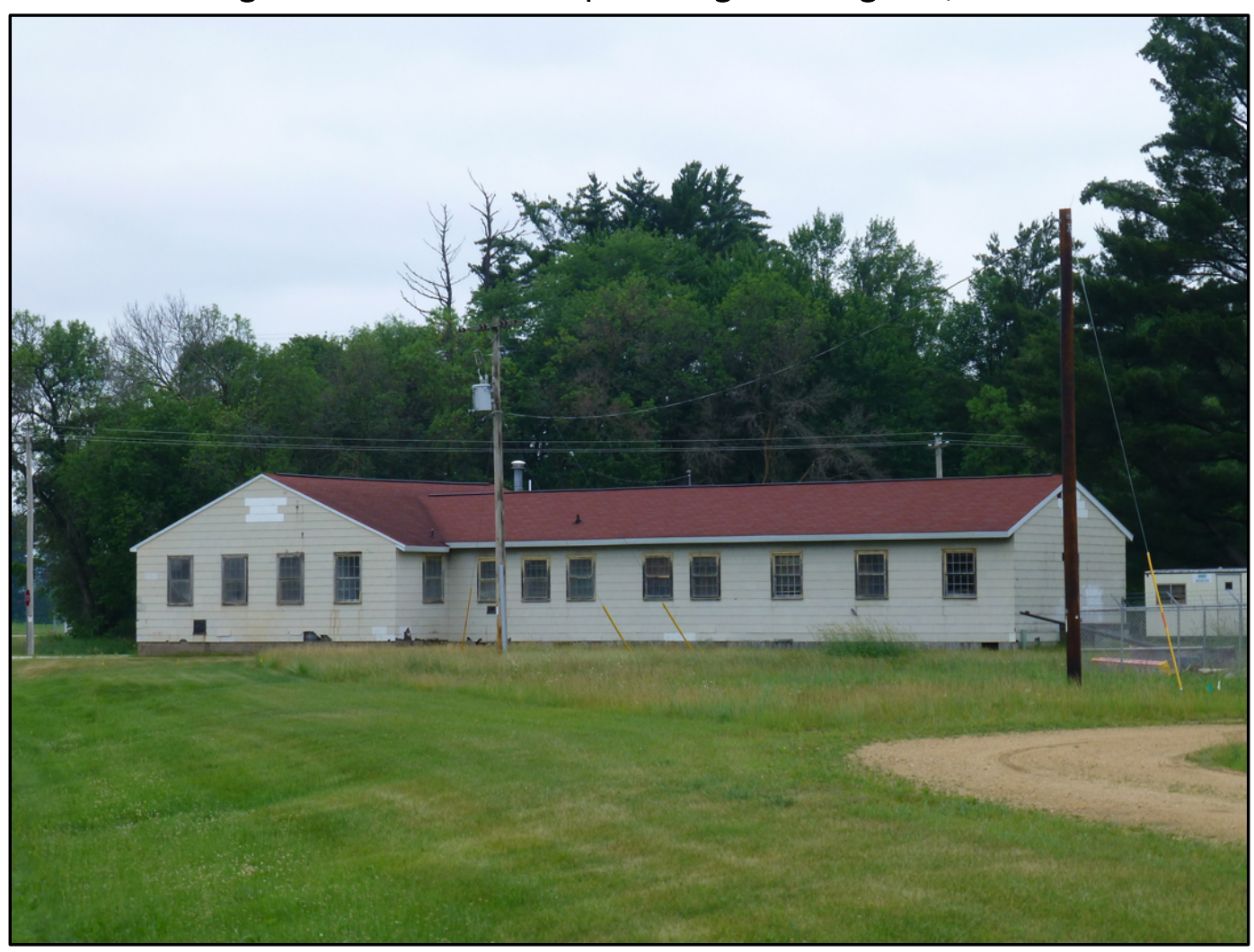

Walls are either open studs, covered with wood planks (see Figure 100), or covered with wall board (see Figure 101). Ceilings are either open like in the apparatus room or covered with wall board and wood battens (see Figures 102 and 103). The 700 and 800-series of WWII temporary buildings were some of the first mass produced buildings in the country. They utilized an early type of wall board manufactured out of fibers and pressed together. Generally, WWII temporary buildings only utilized this wall board in public areas or ceilings. The wall board throughout the building is in FAIR condition but some portions need to be repaired or replaced. The fiberboard is no longer produced but modern gypsum board can replace it as long as the wood battens are retained and reinstalled. 
Figure 100. Wood plank walls and partition in the latrine in Bldg. 550, 2018.

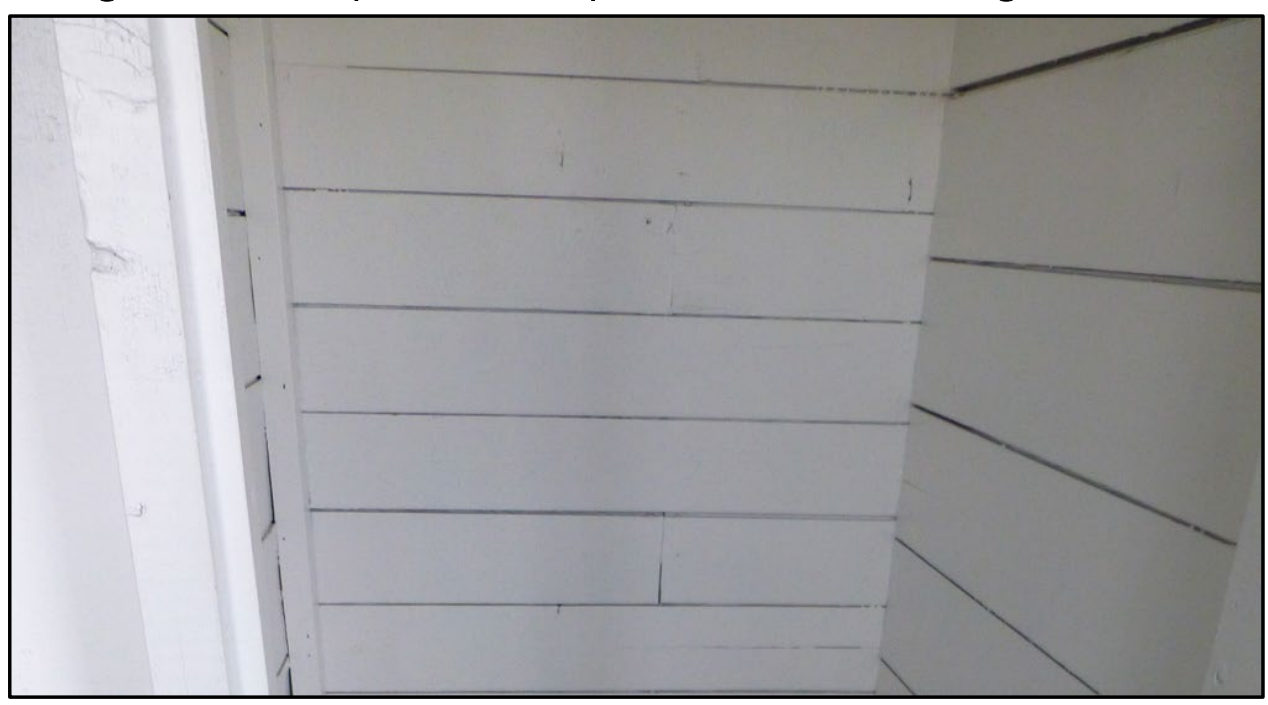

Figure 101. The central hallway with wall board on the wall in Bldg. 550, 2018.

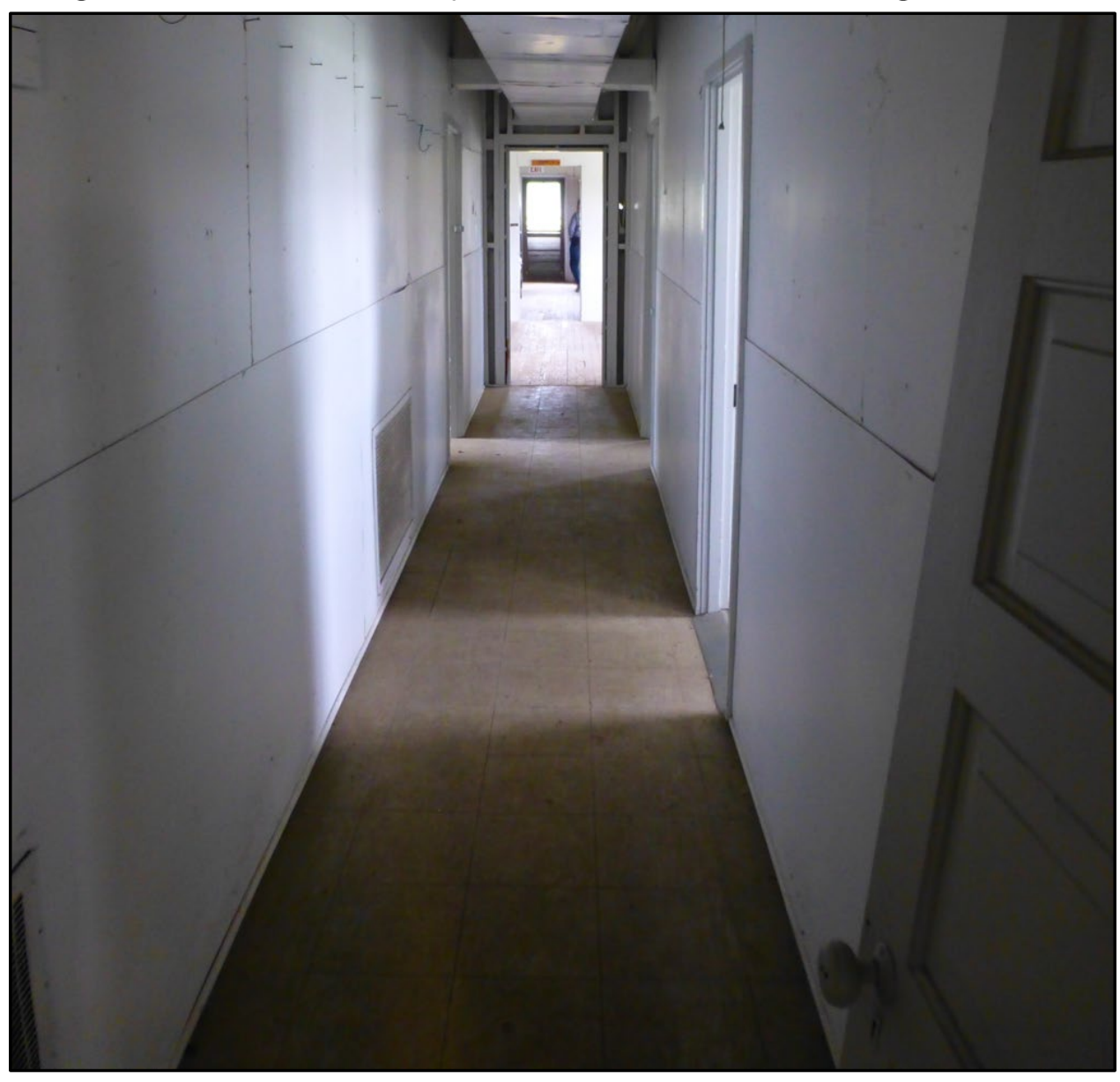


Figure 102. Looking up at original wall board and wood battens on the ceiling in the squad room, 2018.

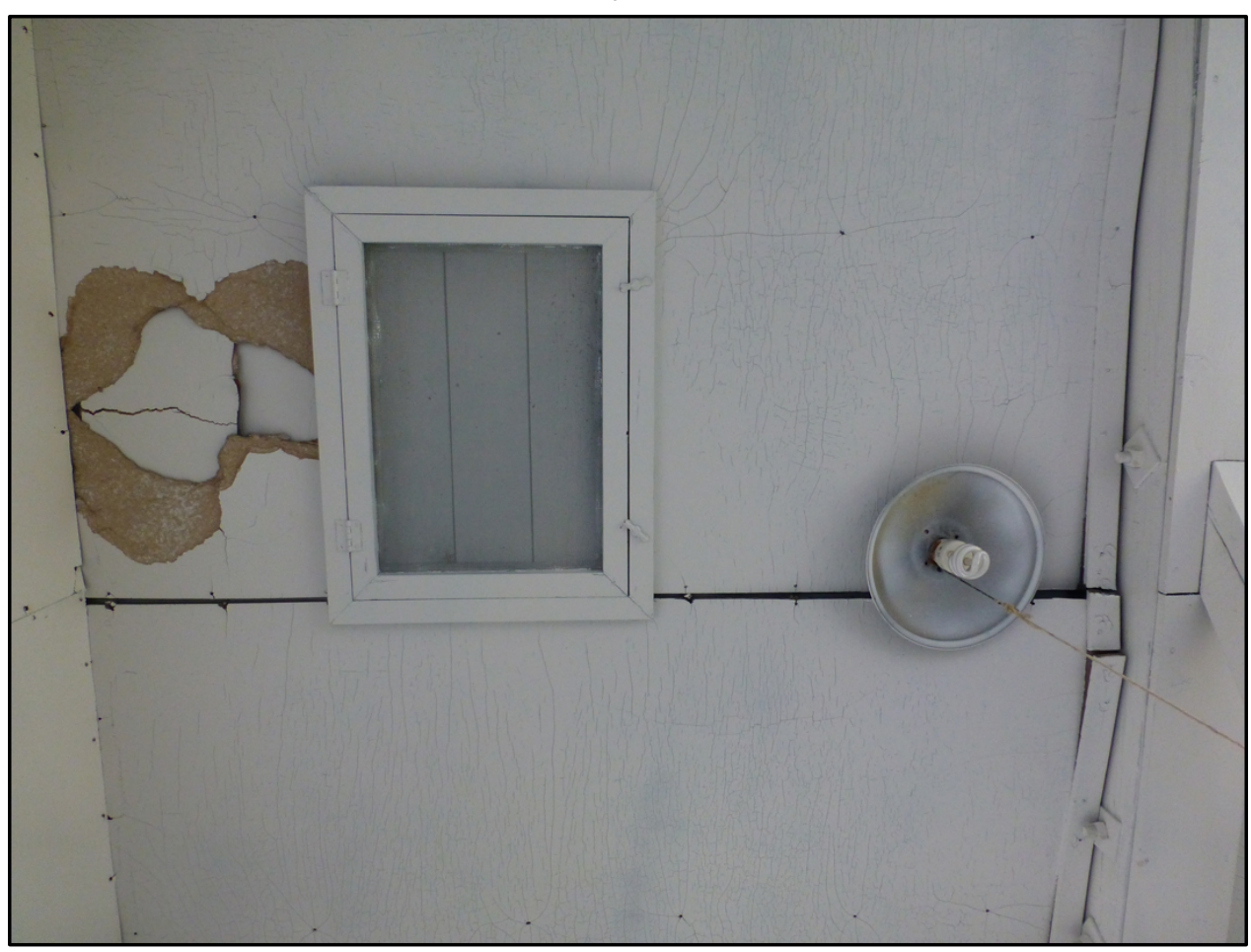

Figure 103. Looking up at original wall board and wood battens on the ceiling in the squad room, 2018.

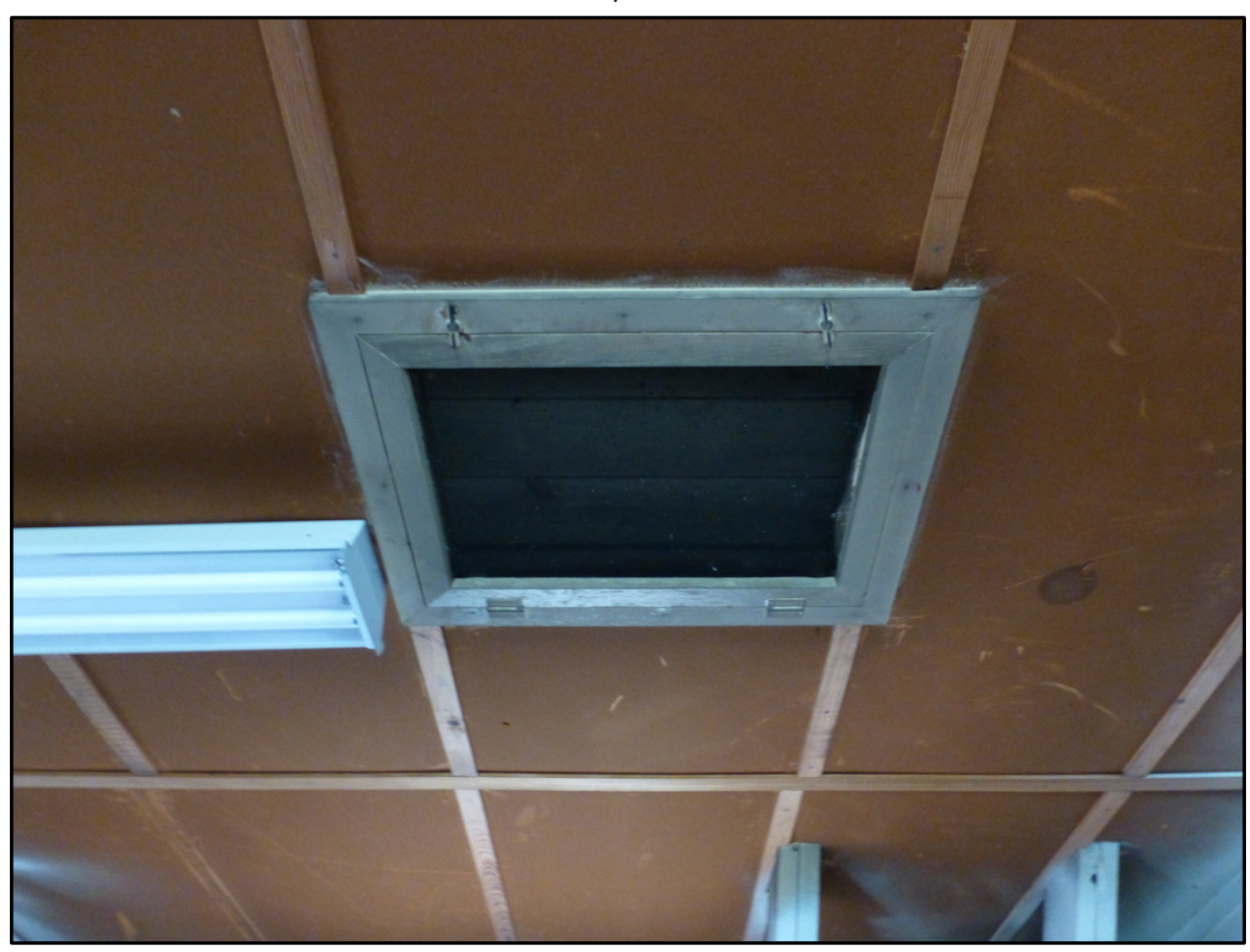


The metal ductwork throughout the building is original (see Figures 104 and 105). As was the case with wall board, WWII temporary buildings were some of the first mass produced buildings to utilize forced hot air furnaces. Because of this, the metal ductwork is a character-defining feature of these WWII-era buildings and needs to be retained.

Figure 104. Looking up at original metal ductwork for the heating system in the squad room, 2018.

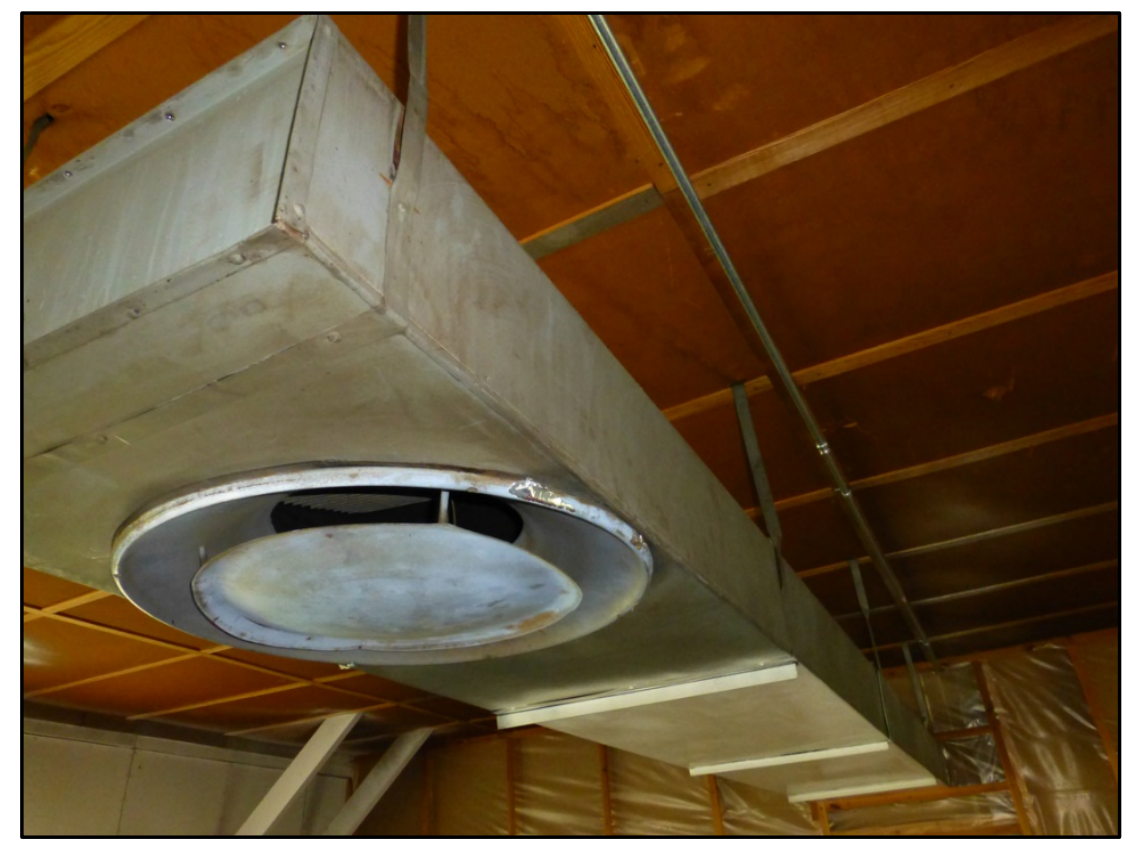

Figure 105. Looking up at original metal ductwork for the heating system in the central hallway, 2018.

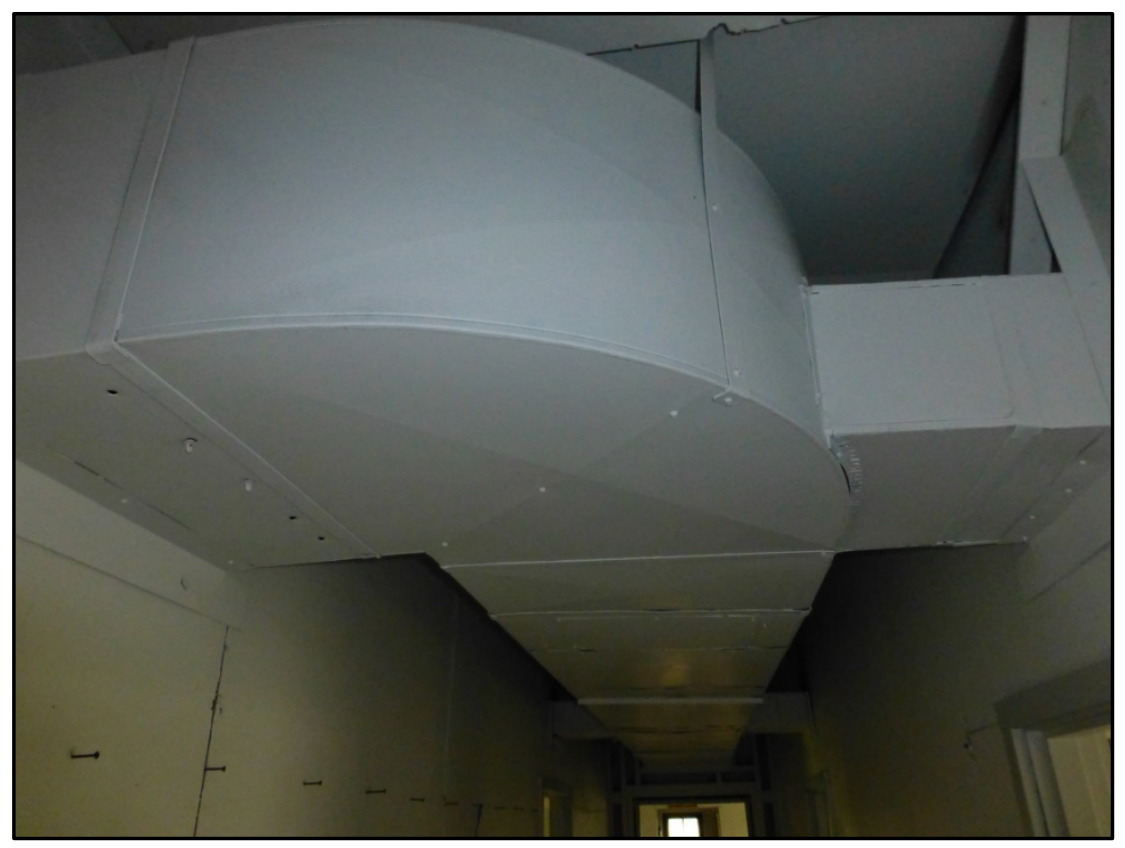




\section{Summary and Recommendations}

Bldg. 550 was erected in 1942 to function as a fire station. The standardized design was developed by the War Department and employed across the country on Army installations which used the 800 Series-type construction plans. The building retains many original elements from the time of its construction, but notable exceptions include a replacement red 3-tab asphalt roof (in-kind), rafter tails and soffits which were originally open now covered with metal, replacement metal overhead doors in the apparatus room, and removed original asbestos shingle with scalloped bottom edge siding.

\subsection{Character-defining features}

In Preservation Brief \#17,38 Nelson reminds readers that the Secretary of the Interior's Standards for the Treatment of Historic Properties $39 \mathrm{em}-$ bodies two important goals: (1) the preservation of historic materials, and (2) the preservation of a building's distinguishing character. Every old building is unique, with its own identity and its own distinctive character. Character refers to all those visual aspects and physical features that comprise the appearance of every historic building. Character-defining elements include the overall shape of the building; its materials, craftsmanship, decorative details, interior spaces, and features; and various aspects of its site and environment.

If the various materials, features, and spaces that give a building its visual character are not recognized and preserved, then essential aspects of its character may be damaged in the process of change.

A list of character-defining features for Bldg. 550 follows. 40

- Massing

- T-shape footprint

- Wood frame structure

38 Lee H. Nelson, National Park Service Preservation Brief 17: Architectural Character-Identifying the Visual Aspect of Historic Buildings as an Aid to Preserving their Character (Washington, DC: U.S. Department of the Interior, National Park Service, 1998), http://www.nps.gov/tps/how-to-preserve/briefs/17architectural-character.htm.

39 Grimmer, Secretary of the Interior's Standards for the Treatment of Historic Properties with Guidelines for the Treatment of Cultural Landscapes.

40 Smith, Determination of NRHP Eligibility for 26 Buildings at Fort McCoy, Wisconsin, 2018, p. 155. 
- Textured asbestos shingle siding with scalloped bottom edge detail (siding was removed in 2019)

- Vertical line definition between each siding piece (siding was removed in 2019)

- Cross-gable roof with 3-tab red asphalt shingles

- Concrete pier foundation system (living area section) and concrete block wall/slab foundation system (heater room and apparatus room)

- Multi-pane wood double-hung windows

○ Wood screens

- Wood trim size and profile around window and door openings

- Wood panel and divided-light

- Wood interior screen doors

- Brick furnace stack (removed above roofline)

- Exterior light fixtures (replaced)

- Open bay apparatus room with concrete floor and walls clad with wall board

- Wood and glass overhead doors (replaced with metal overhead doors)

- Living area with central corridor

- Toilet room with fixtures such as urinals, sinks, mirrors, light fixtures, shower stall, concrete floor, and wood plank walls

- Wood floor

- Walls of either wood studs or covered in wall board

- Ceiling covered with wall board and wood battens

- Suspended mechanical system and ductwork in the squad room and central corridor

- Interior wood panel doors

- Interior mop sinks and drinking fountains in the apparatus room

\subsection{Treatment}

The Secretary of the Interior is responsible for establishing professional standards and providing advice on the stewardship of cultural resources listed on or as eligible for the NRHP. The Secretary's standards describe four basic approaches to the treatment of historic landscapes.

\subsubsection{Restoration approach}

Restoration is the act or process of accurately depicting the form, features, and character of a property as it appeared at a particular period in time. 
This process includes reconstruction of missing features from the restoration period and removal of features from all other periods. The approach can be considered only when the property's significance during a particular period of time outweighs the loss of extant elements from other historical periods, and when there is substantial physical and documentary evidence for the work, and when contemporary alterations and additions are not planned.

While Bldg. 550 is a highly intact building from WWII, restoration is not an appropriate approach for it since the building is vacant and no longer a fire station and is not scheduled for restoration into a museum building.

\subsubsection{Reconstruction approach}

Reconstruction is the act or process of using new construction to depict a non-surviving site, landscape, building, structure, or object as it appeared at a specific period of time and in its historic location. The approach is appropriate only when the property's significance during a particular period of time outweighs the potential loss of extant features that characterize other historical periods. In addition, there must be substantial physical and documentary evidence for the work, and the work must be clearly identified as a contemporary re-creation.

While Bldg. 550 is a highly intact building from WWII, reconstruction is a not a viable path for maintaining it.

\subsubsection{Preservation approach}

Preservation involves applying measures to sustain the existing form, integrity, and materials of a historic property. This approach focuses on stabilizing and protecting extant historic resources, rather than replacing missing elements. It is appropriate when a historic property is essentially intact and does not require extensive repair or replacement; depiction at one particular period of time is not appropriate; and when continuing or new use does not require additions or alterations.

Preservation is a management treatment for Bldg. 550 due to the large amount of intact resources; however, this treatment may not be conducive to reutilizing the building for new uses. 


\subsubsection{Rehabilitation approach}

Rehabilitation allows repairs, alterations, and additions necessary to enable a compatible use for a property as long as the portions or features which convey the historical, cultural, or architectural values are preserved. This approach is appropriate when depiction at one particular period of time is not appropriate, repair or replacement of deteriorated features is necessary, and alterations or additions are needed for a new use.

Rehabilitation is appropriate for Bldg. 550 as a successful reuse of it will move the building from a vacant status to an occupied status. While it may never see use a fire station again, it is highly likely that this building can serve again as administration space or a maintenance building.

\subsection{Management issues and recommendations}

Bldg. 550 is federally owned by Fort McCoy, and the building was determined eligible for the NRHP in 2018. As such, Fort McCoy consults for all undertakings that affect the building with the State of Wisconsin, Wisconsin Historical Society, which serves as the State Historic Preservation Officer (SHPO) for consultation purposes.

Current issues include the following:

- Vacant building

- Removal of original historic asbestos siding and covering of entire building with building wrap

- Open rafters and soffits covered in metal

- Lack of recent painting

- Windows need repairing

- Screens need repairing

- Doors need repairing

- Inappropriate metal doors on the west side

- Landing on east side needs to be rebuilt

- Inappropriate exterior light fixtures

- Wall and ceilings need repairing

- Bathroom is not useable

- Unknown heat and air-conditioning status. 


\subsection{Historic building recommendations}

The following actions are recommended to address the issues outlined above in section 13.3 and should be written into any renovation contract for Bldg. 550:

- Determine a new use for Bldg. 550 .

- Reside Bldg. 550 with shingle siding with scalloped bottom edge.

- Refurbish all windows, doors, screens, and screen doors according to the treatment measures.

- Remove all metal covering rafter tails and soffits and paint wood according to the treatment measures.

- Replace the three metal doors on the west side of the building with wood doors that recreate the original ones. (If the building is going to be adaptively reused into administrative space, it is still recommended that these doors be recreated but that they do not open allowing the former apparatus bay to be used as office space.)

- Rebuild the landing on the east side using the original plans. (Americans with Disabilities Act [ADA] ramps will need to be added but should not pose an issue if designed under consultation.)

- Replace inappropriate exterior light fixtures with ones that match the original plans.

- Walls and ceilings need to be repaired using in-kind materials, i.e., wood for wood, wall board for wall board, wood batten for wood batten.

- The current latrine can be redesigned to ADA standards while keeping some elements of its WWII period of significance.

- Create a new heating, ventilating, and air-conditioning (HVAC) system that uses the heater room and the extant metal ducting as much as possible. 


\section{Acronyms and Abbreviations}

\begin{tabular}{|l|l|}
\hline Abbreviation & Term \\
\hline ACHP & Advisory Council on Historic Preservation \\
\hline ADA & Americans with Disabilities Act \\
\hline DoD & Department of Defense \\
\hline DPW & Directorate of Public Works \\
\hline ERDC & Engineer Research and Development Center \\
\hline HABS & Historic American Buildings Survey \\
\hline HVAC & Heating, Ventilating, and Air-Conditioning \\
\hline NCO & Non-Commissioned Officer \\
\hline NCSHPO & National Council of State Historic Preservation Officers \\
\hline NHPA & National Historic Preservation Act \\
\hline NRHP & National Register of Historic Places \\
\hline PMOA & Programmatic Memorandum of Agreement \\
\hline SHPO & State Historic Preservation Officer \\
\hline WWII & World War II \\
\hline
\end{tabular}




\section{Conversion Factors}

\begin{tabular}{|c|c|c|}
\hline Multiply & By & To Obtain \\
\hline feet & 0.3048 & meters \\
\hline inches & 0.0254 & meters \\
\hline miles (U.S. statute) & $1,609.347$ & meters \\
\hline square feet & 0.09290304 & square meters \\
\hline square inches & $6.4516 \mathrm{E}-04$ & square meters \\
\hline square miles & $2.589998 \mathrm{E}+06$ & square meters \\
\hline yards & 0.9144 & meters \\
\hline
\end{tabular}




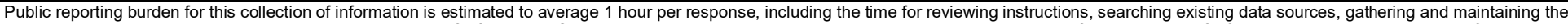

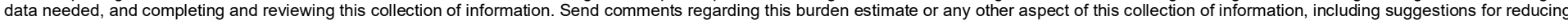

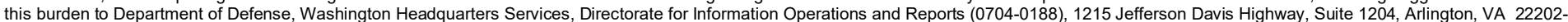

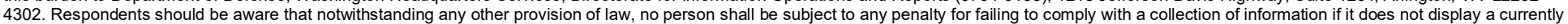
valid OMB control number. PLEASE DO NOT RETURN YOUR FORM TO THE ABOVE ADDRESS.
1. REPORT DATE (DD-MM-YYYY)
2. REPORT TYPE
$11 / 01 / 2020$
Final Technical Report (TR)

\section{TITLE AND SUBTITLE}

Fort McCoy, Wisconsin Building 550 Maintenance Plan

August S. Fuelberth, Adam D. Smith, and Sunny E. Adams

3. DATES COVERED (From - To)

5a. CONTRACT NUMBER

5b. GRANT NUMBER

5c. PROGRAM ELEMENT

\section{5d. PROJECT NUMBER}

485435

5e. TASK NUMBER

5f. WORK UNIT NUMBER

8. PERFORMING ORGANIZATION REPORT NUMBER

ERDC/CERL TR-20-11

U.S. Army Engineer Research and Development Center (ERDC)

Construction Engineering Research Laboratory (CERL)

PO Box 9005,

Champaign, IL 61826-9005

\section{SPONSORING / MONITORING AGENCY NAME(S) AND ADDRESS(ES)}

10. SPONSOR/MONITOR'S ACRONYM(S)

U.S. Army Garrison Fort McCoy

100 East Headquarters Road

Fort McCoy, WI 54656-5263

11. SPONSOR/MONITOR'S REPORT NUMBER(S)

\section{DISTRIBUTION / AVAILABILITY STATEMENT}

Approved for public release; distribution is unlimited.

\section{SUPPLEMENTARY NOTES}

\section{ABSTRACT}

Building 550 (former World War II fire station) is located on Fort McCoy, Wisconsin, and was recommended eligible for the National Register of Historic Places (NRHP) in 2018 (Smith and Adams 2018). The building is currently vacant. It is an intact example of an 800 Series World War II fire station with character-defining features of its period of significance from 1939 to 1946 on its exterior and interior. All buildings, especially historic ones, require regular planned maintenance and repair. The most notable cause of historic building element failure and/or decay is not the fact that the historic building is old, but rather it is caused by incorrect or inappropriate repair and/or basic neglect of the historic building fabric. This document is a maintenance manual compiled with as-is conditions of construction materials of Building 550. The Secretary of Interior Guidelines on rehabilitation and repair per material are discussed to provide the cultural resources manager at Fort McCoy a guide to maintain this historic building. This report satisfies Section 110 of the National Historic Preservation Act (NHPA) of 1966 as amended and will help the Fort McCoy Cultural Resources Management office to manage this historic building.

\section{SUBJECT TERMS}

Fort McCoy (Wis.)-Historic buildings, Historic buildings-Maintenance and repair, Historic preservation, Fire stations

\section{SECURITY CLASSIFICATION OF:}

\section{a. REPORT}

Unclassified

\section{b. ABSTRACT \\ Unclassified}

\section{LIMITATION OF ABSTRACT}

\section{c. THIS PAGE}

Unclassified
SAR
18. NUMBER OF PAGES

227 19a. NAME OF RESPONSIBLE PERSON

19b. TELEPHONE NUMBER

(include area code) 